\title{
Aplicaciones de soluciones no homogéneas \\ de las ecuaciones de Einstein a problemas cosmológicos
}

Florencia Anabella Teppa Pannia

Director: Dr. Santiago Esteban Perez Bergliaffa Codirector: Dr. Gustavo Esteban Romero

Tesis presentada para optar por el grado de DOCTORA EN ASTRONOMÍA

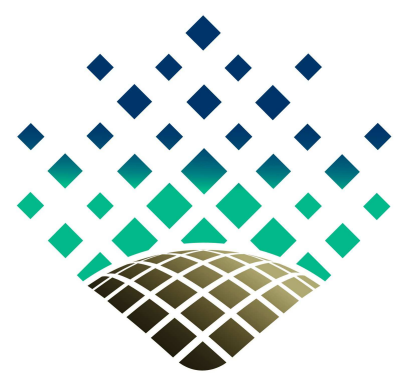

Facultad de Ciencias Astronómicas y Geofísicas

UNIVERSIDAD NACIONAL DE LA PLATA 



\section{Resumen}

La expansión acelerada del universo es una de las características más importantes del modelo cosmológico estándar. La evidencia observacional que sustenta tal expansión, interpretada dentro de dicho modelo (basado en la Teoría de la Relatividad General junto las hipótesis de homogeneidad e isotropía), indica que el Universo estaría dominado por la comúnmente llamada "energía oscura", en una proporción aproximada de $7 / 3$ con relación a la materia oscura. Esta nueva forma de materia debería poseer características peculiares: su densidad aumentaría con el volumen y su distribución sería espacialmente homogénea. Tales atributos, muy alejados de aquellos que caracterizan a la materia ordinaria, sugieren que es necesario modificar el modelo estándar.

El tema de investigación de esta tesis son las soluciones exactas no homogéneas de las ecuaciones de Einstein y sus aplicaciones a diferentes problemas de interés cosmológico. Con las motivaciones presentadas anteriormente, el objetivo general del trabajo es estudiar modelos cosmológicos no homogéneos, viables como alternativa para describir las observaciones astronómicas sin la necesidad de recurrir a la incorporación de energía oscura.

En particular, nos concentraremos en estudiar aquellas soluciones con simetría esférica, a saber, la solución de Lemaître-Tolman-Bondi, con un fluido perfecto sin presión como fuente, y la solución de Lemaître, que admite la descripción de un fluido con presión no nula. Discutiremos también algunos aspectos de la solución de Székeres.

Entre las aplicaciones a problemas cosmológicos que abordaremos en esta tesis, podemos destacar:

1. El estudio de la variación temporal del corrimiento al rojo cosmológico debida a la tasa variable de expansión del universo (redshift drift). Esta cantidad, potencialmente medible con la tecnología actual, ofrecería la posibilidad de distinguir modelos cosmológicos. Es importante entonces estudiar las predicciones teóricas para este observable en diferentes escenarios, con el fin de hacer futuras comparaciones con las observaciones.

2. El desarrollo de la inflación en presencia de regiones no homogéneas. Una de las hipótesis importantes en la que se basan los modelos de inflación es la de suponer que cualquier irregularidad inicial es homogeneizada debido a la violenta expansión que caracteriza a la etapa inflacionaria. Si bien existen argumentos teóricos que justifican esta hipótesis, no hay una prueba definitiva de que la inflación tenga efectivamente esta propiedad para cualquier tipo de escenario inicial. Con esta motivación, estudiaremos si una región del espacio-tiempo puede presentar una etapa de inflación en un ambiente no homogéneo, con el objetivo de comprobar si alguna distribución primordial no homogénea de materia o radiación podría inhibir el avance de la inflación.

3. Modelos con distribución inicial no homogénea de materia y radiación. Los escenarios cosmológicos con dos fluidos no homogéneos han sido propuestos para modelar los efectos de la radiación sobre la evolución temprana de la geometría del universo. En este contexto, estudiaremos la evolución de un modelo de dos fluidos con simetría esférica (en donde la radiación y la materia son no comóviles). En particular, analizaremos los efectos de la presión anisótropa de la radiación sobre la evolución de la geometría.

Concluiremos el trabajo con una discusión general sobre los modelos no homogéneos y las perspectivas observacionales que permitan distinguir los diferentes escenarios cosmológicos. 


\section{Summary}

The accelerated expansion of the universe is one of the most important features of the Standard Cosmological Model. The observational evidence that support such expansion, interpreted in the context of that model (which is based on General Relativity and the hypothesis of homogeneity and isotropy), indicates that the universe is dominated by the so-called "dark energy" in a proportion $7 / 3$ with respect to dark matter. This unknown fluid presents peculiar features: a negative pressure (that is, tension) and a smooth distribution (because it is not collected into potencial wells). This scenario suggests that the standar model must be revised.

The topic of this thesis is the study of inhomogeneous exact solutions of Einstein's equations, and their applications to solve different cosmological problems. Motivated by the dark energy problem, the aim of our work is to investigate inhomogeneous cosmological models as a viable alternative to describe astronomical observations without including dark energy.

In particular, we are interesting in those solutions with spherical symmetry, such as the Lemaître-Tolman-Bondi (LTB) and the Lemaître solutions, which describe perfect fluids of dust and radiation, respectively. We will also discuss some aspects of the Székeres solution.

We will apply the inhomogeneous solutions to investigate three specific cosmological issues:

1. The redshift drift in cosmological models. Among the cosmological observables, the redshift drift (that is, the rate of change of the cosmological redshift) has been studied with increasing interest due to its potential to distinguish between different cosmological models. Since there are good prospects to observe the redshift drift in the next decade, it is important to develop theoretical approaches that allow the forecast of its features in a given cosmological model.

2. Inhomogeneous initial conditions for inflation. Although inflation is specifically designed to solve some of the problems of the Standard Cosmological Model, it is not free of its own problems. Among these, perhaps the most relevant ones are those related to the beginning of inflation in the presence of inhomogeneities, and the evolution of an inflating region in an inhomogeneous ambient. Motivated by this scenario, we will study generic inhomogeneous initial conditions for inflation, in both dust and radiation inhomogeneous backgrounds.

3. Spherically symmetric void models with dust and radiation. Spherically symmetric void models with dust and (non-comoving) radiation have been carefully studied with the aim of describing current cosmological observations without using dark energy. In this context, we will study the effects due to the anisotropic pressure of the radiation in the early evolution of the geometry.

We will conclude with some remarks on inhomogeneous cosmological models and the observational perspectives that would allow to distinguish between cosmologies in the near future. 


\section{Lista de publicaciones}

Trabajos publicados en revistas internacionales con arbitraje

- "Constraining $f(R)$ theories with cosmography". F. A. Teppa Pannia \& S. E. Perez Bergliaffa.

Journal of Cosmology and Astroparticle Physics, Vol. 8, p. 30 (2013).

\section{Trabajos en preparación}

- "Evolution of Cosmic Bubbles in Inhomogeneous Backgrounds". F. A. Teppa Pannia \& S. E. Perez Bergliaffa.

- "A Covariant Approach to the Redshift Drift". S. E. Perez Bergliaffa \& F. A. Teppa Pannia.

- "Anisotropic Radiation Pressure in Two-Fluid 'Void' Models". F. A. Teppa Pannia \& S. E. Perez Bergliaffa (en colaboración con C. Clarkson, M. Regis \& W. C. Lim).

\section{Publicaciones complementarias}

- "Structure of neutron stars in R-squared gravity". M. Orellana, F. García, F. A. Teppa Pannia \& G. E. Romero. General Relativity and Gravitation, Vol. 45, p. 771-783 (2013).

- "Structure of Compact Stars in R-squared Palatini Gravity". F. A. Teppa Pannia, F. García, S. E. Perez Bergliaffa, M. Orellana \& G. E. Romero. Enviado para ser sometido a arbitraje (febrero 2016).

\section{Trabajos publicados en actas de congresos con arbitraje}

- "Inflation in Inhomogeneous Spacetimes: Bubbles Evolution". F. A. Teppa Pannia \& S. E. Perez Bergliaffa. Proceedings of the GRACo II Argentinian-Brazilian Meeting (abril 2014).

- "Structure of Compact Stars in Palatini $f(R)$ Gravity". F. García, F. A. Teppa Pannia, S. E. Perez Bergliaffa, M. Orellana \& G. E. Romero. Proceedings of the GRACo II Argentinian-Brazilian Meeting (abril 2014).

- "A cosmographic Approach to the Redshift Drift in FLRW Models". F. A. Teppa Pannia \& S. E. Perez Bergliaffa. Boletín Anual de la Asociación Argentina de Astronomía, Vol. 55, p. 499-503 (2012).

- "Compact Stars in R-squared Gravity". F. García, F. A. Teppa Pannia, M. Orellana \& G. E. Romero. Boletín Anual de la Asociación Argentina de Astronomía, Vol. 55, p. 499-503 (2012).

- "Redshift Drift in LTB Models". F. A. Teppa Pannia \& S. E. Perez Bergliaffa. Proceedings of the GRACo I Argentinian-Brazilian Meeting (octubre, 2011). 


\section{Lista de trabajos presentados en congresos y escuelas internacionales}

- "Anisotropic Radiation Pressure in Two-Fluid 'Void' Models".

F. A. Teppa Pannia \& S. E. Perez Bergliaffa (contribución oral).

III Cosmo Sur. Córdoba, Argentina. Agosto de 2015.

- "Bubble Evolution in Inflation Scenarios".

F. A. Teppa Pannia \& S. E. Perez Bergliaffa (contribución mural).

III Jayme Tiomno School of Cosmology. Rio de Janeiro, Brasil. Agosto de 2014.

- "Inflation in Inhomogeneous Spacetimes: Bubbles Evolution".

F. A. Teppa Pannia \& S. E. Perez Bergliaffa (contribución mural).

Second Argentinian-Brazilian Meeting of Gravitation, Relativistic Astrophysics and Cosmology. Buenos Aires, Argentna. Abril de 2014.

- "Structure of Compact Stars in Palatini $f(R)$ Gravity".

F. García, F. A. Teppa Pannia, S. E. Perez Bergliaffa, M. Orellana \& G. E. Romero (contribución mural).

Second Argentinian-Brazilian Meeting of Gravitation, Relativistic Astrophysics and Cosmology. Buenos Aires, Argentna. Abril de 2014.

- "Cosmographical Redshift Drift in FLRW Models".

F. A. Teppa Pannia \& S. E. Perez Bergliaffa (contribución mural).

V Challenges of New Physics in Space. Rio de Janeiro, Brasil. Abril de 2013.

- "A cosmographic Approach to the Redshift Drift in FLRW Models".

F. A. Teppa Pannia \& S. E. Perez Bergliaffa (contribución oral).

55 Reunión Anual de la Asociación Argentina de Astronomía. Mar del Plata, Argentia. Septiembre de 2012.

- "Compact Stars in R-squared Gravity".

F. García, F. A. Teppa Pannia, M. Orellana \& G. E. Romero (contribución mural).

55 Reunión Anual de la Asociación Argentina de Astronomía. Mar del Plata, Argentia. Septiembre de 2012.

- "Redshift Drift in LTB Models".

F. A. Teppa Pannia \& S. E. Perez Bergliaffa (contribución mural).

Summer School on Cosmology (ICTP). Trieste, Italia. Octubre de 2011.

- "An Approach for the Redshift Drift in LTB Models".

F. A. Teppa Pannia \& S. E. Perez Bergliaffa (presentación mural).

First Argentinian-Brazilian Meeting on Gravitation, Relativistic Astrophysics and Cosmology. Foz de Iguaçú. Octubre de 2011. 


\section{Índice general}

1. Introducción 3

1.1. Breve introducción al modelo cosmológico estándar . . . . . . . . . . . . . 4

1.1.1. Construcción de un modelo isótropo y homogéneo . . . . . . . . . . . . 4

1.1.2. El modelo $\Lambda$ CDM . . . . . . . . . . . . . . . . . . 8

1.2. Problemas del modelo cosmológico estándar . . . . . . . . . . . . . . . 11

1.2.1. Energía oscura . . . . . . . . . . . . . . . . . . 13

1.2.2. Inflación . . . . . . . . . . . . . . . . . . 15

1.3. Modelos cosmológicos no homogéneos . . . . . . . . . . . . 16

1.4. Objetivos de esta tesis . . . . . . . . . . . . . . . . . . 18

2. Soluciones no homogéneas de las ecuaciones de Einstein 25

2.1. La solución de Lemaître-Tolman-Bondi . . . . . . . . . . . . . . . . . . . 26

2.1.1. Condiciones de regularidad . . . . . . . . . . . . . . . . . . 29

2.1.2. Propagación de la luz en la geometría de LTB . . . . . . . . . . . . . . 32

2.1.3. Aplicaciones de la solución de LTB . . . . . . . . . . . . . . . . . . 33

2.2. La solución de Lemaître . . . . . . . . . . . . . . . . . . . . . . . . . . . 33

2.3. La solución de Székeres . . . . . . . . . . . . . . . . . . . . . . . . 34

3. Cosmología a tiempo real: la variación temporal del redshift cosmológico 43

3.1. Cosmología a tiempo real . . . . . . . . . . . . . . . 44

3.1.1. Redshift drift como test para distinguir modelos cosmológicos . . . . . . 45

3.2. Formulación covariante del redshift drift $(z \ll 1) \ldots \ldots \ldots$. . . . . . . 46

3.2.1. Aplicaciones a diferentes escenarios cosmológicos . . . . . . . . . . . . 48

3.2.2. Discusión . . . . . . . . . . . . . . . . . 55

3.3. Aproximación cosmográfica del redshift drift $(z \ll 1) \ldots \ldots \ldots \ldots$. . . . . 57

3.4. Discusión . . . . . . . . . . . . . . . . . . . 60 60

$\begin{array}{lll}\text { 4. Inflación y regiones no homogéneas } & 67\end{array}$

4.1. Modelos inflacionarios . . . . . . . . . . . . . . . . 68

4.1.1. El mecanismo básico de la inflación . . . . . . . . . . . . . . . . . . 68

4.1.2. Condiciones iniciales para la inflación . . . . . . . . . . . . 70

4.2. El formalismo de la cáscara delgada . . . . . . . . . . . . . . . . . . 72

4.2.1. Cálculo numérico de la evolución de la burbuja . . . . . . . . . . . . 77

4.2.2. Condiciones iniciales para la evolución de la burbuja . . . . . . . . . . . 77

4.3. Evolución en un fondo homogéneo: polvo vs. radiación . . . . . . . . . . . . . 79

4.4. Evolución en fondos no homogéneos . . . . . . . . . . . . . . . . . 82 
4.4.1. Evolución en un fondo no homogéneo de polvo . . . . . . . . . . . . . . 82

4.4.2. Evolución en un fondo no homogéneo de radiación . . . . . . . . . . . . 84

4.5. Discusión general . . . . . . . . . . . . . . . . . . 88

$\begin{array}{ll}\text { 5. Modelos de void con dos fluidos } & 93\end{array}$

5.1. Los modelos de void . . . . . . . . . . . . . . . . . . . . . . . 94

5.1.1. La importancia de la radiación en los modelos de void . . . . . . . . . . 96

5.1.2. Un modelo de dos fluidos no comóviles . . . . . . . . . . . . . . . . 97

5.2. Descripción del modelo de dos fluidos no homogéneos . . . . . . . . . . . . 98

5.2.1. Caracterización de la geometría . . . . . . . . . . . . . . . . 98

5.2.2. Caracterización de los fluidos . . . . . . . . . . . . . . . . . . 99

5.2.3. Modelo sin presión anisótropa de la radiación . . . . . . . . . . . . . . 100

5.2.4. Momentos multipolares de la radiación . . . . . . . . . . . . . . . . . . . 101

5.2.5. Modelo de dos fluidos generalizado . . . . . . . . . . . . . . . . . 103

5.2.6. Cálculo del redshift y de distancias cosmológicas . . . . . . . . . . . . 103

5.3. Implementación numérica . . . . . . . . . . . . . . . . . . . . . . 105

5.3.1. Condiciones de borde y regularidad en el centro de simetría . . . . . . . 105

5.3.2. Condiciones iniciales . . . . . . . . . . . . . . . . . . . . 108

5.4. Resultados y discusión . . . . . . . . . . . . . . . . . . 110

$\begin{array}{ll}\text { 6. Conclusiones } & 117\end{array}$

$\begin{array}{ll}\text { A. Breve introducción a los formalismos covariantes } & \mathbf{1 2 1}\end{array}$

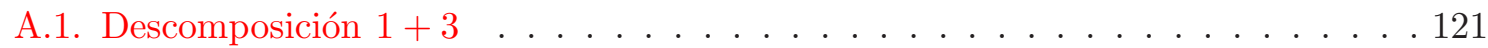

A.2. Tétradas en modelos cosmológicos . . . . . . . . . . . . . . . . . . 124

B. Propagación de la luz en modelos de LTB fuera del centro de simetría 129

B.1. Método I . . . . . . . . . . . . . . . . . . . . . . . . . 129

B.2. Método II . . . . . . . . . . . . . . . . . . . . . . . . . 131

C. Otras soluciones de las ecuaciones de Einstein de interés cosmológico 135

C.1. La solución de Bianchi tipo I . . . . . . . . . . . . . . . . . . . 135

C.2. La solución de Stephani . . . . . . . . . . . . . . . . . . . . 137

C.2.1. Modelos cosmológicos de Stephani . . . . . . . . . . . . . . . . 139

D. El Redshift drift: un potencial observable para distinguir cosmologías $\quad 141$

E. El redshift drift cosmográfico en teorías $f(R) \quad \mathbf{1 4 5}$

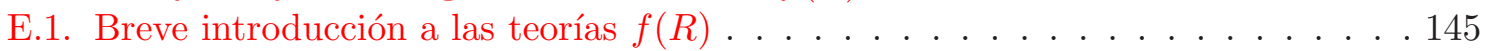

E.2. Tratamiento cosmográfico para teorías $f(R) \ldots \ldots \ldots \ldots \ldots$

E.2.1. Ejemplo 1: modelo de Hu \& Sawicki . . . . . . . . . . . . . . . . . . 150

E.2.2. Ejemplo 2: gravedad exponencial . . . . . . . . . . . . . . . 153

E.2.3. Discusión . . . . . . . . . . . . . . . . . . . . 154

$\begin{array}{ll}\text { Bibliografía } & 159\end{array}$ 
"Si las galaxias se alejan, el enrarecimiento del universo es compensado por la formación de nuevas galaxias compuestas de materia que se crea ex novo. Para mantener estable la densidad media del universo, basta que se forme un átomo de hidrógeno cada 250 millones de años por cada 40 centímetros de espacio en expansión.

(Esta teoría, llamada del "estado estacionario", ha sido contrapuesta a otra hipótesis de que el universo fue originado, en un momento preciso, por una gigantesca explosión.)

Yo era chico y no me había dado cuenta - contó Qfwfq-. Los átomos de hidrógeno los conocía uno por uno, y cuando aparecía uno nuevo lo sabía. En los últimos tiempos de mi infancia para divertirnos sólo había en el universo átomos de hidrógeno, y no hacíamos más que jugar con ellos, yo y otro chico de mi edad llamado Pfwfp.

¿Cómo era el juego? Es fácil de explicar. Como el espacio es curvo, a lo largo de la curva hacíamos correr los átomos como bolitas, y el que mandaba más lejos su átomo ganaba. Al dar el golpe al átomo había que calcular bien los efectos, las trayectorias, saber aprovechar los campos magnéticos y los campos de gravitación, sino la pelotita salía fuera de la pista y quedaba eliminada de la competencia. (...)"

Juegos Sin Fin

Las Cosmicómicas (1965)

Ítalo Calvino 


\section{Capítulo 1}

\section{Introducción}

La cosmología es la rama de la astronomía que estudia el origen, la estructura y la evolución del universo. A pesar de la complejidad que en principio representa el desafío de modelar el universo como un todo, es posible comenzar por el estudio de modelos con ciertas simplificaciones, que puedan ser cotejados con las observaciones astronómicas actuales.

Los pilares necesarios para construir un modelo cosmológico son, esencialmente, una teoría de la gravitación y una descripción adecuada del contenido de materia y energía del universo. Asimismo, la complejidad de las ecuaciones puede requerir eventuales simplificaciones, basadas principalmente en las simetrías observadas en la distribución de materia del cosmos. Estos ingredientes componen el llamado modelo cosmológico estándar, que ha sido desarrollado en gran parte durante los últimos cincuenta años. Basado en la Teoría de la Relatividad General y las hipótesis de homogeneidad e isotropía a grandes escalas (Gpc), este modelo describe diferentes etapas de la evolución del universo en muy buen acuerdo con distintos conjuntos de evidencias observacionales, abarcando aproximadamente un intervalo temporal de 57 órdenes de magnitud (desde $10^{-38}$ de fracción de segundo después del comúnmente llamado big-bang, hasta la edad actual del universo, estimada en $13 \times 10^{9}$ años).

A pesar de este escenario exitoso, el modelo cosmológico estándar no está exento de problemas, que merecen atención considerable. Entre ellos, nos interesa mencionar: (i) los asociados a la llamada "energía oscura" (un tipo de fluido desconocido en la naturaleza, con características intrínsecas peculiares, que representaría cerca del $70 \%$ del contenido de materia total del universo y dominaría la dinámica actual del cosmos); y (ii) aquellos relativos a las condiciones iniciales que deben imponerse para que una etapa de expansión acelerada (conocida como "inflación") haya tenido lugar durante los tiempos más tempranos de la evolución del universo.

En este contexto, las soluciones exactas no homogéneas de las ecuaciones de Einstein han sido estudiadas como una alternativa viable que pueda dar respuesta a los problemas mencionados anteriormente. En particular, las soluciones con simetría esférica han servido para construir modelos cosmológicos que no precisan incorporar la energía oscura en la descripción del cosmos, así como también para estudiar las condiciones iniciales de escenarios de inflación.

Presentamos en este capítulo una breve introducción al modelo cosmológico estándar, haciendo hincapié en los aspectos problemáticos que motivaron el trabajo desarrollado en esta tesis. Los objetivos concretos de dicho trabajo, y una introducción a los problemas abordados a partir de soluciones no homogéneas de las ecuaciones de Einstein, son expuestos al final del capítulo. 


\subsection{Breve introducción al modelo cosmológico estándar}

\subsubsection{Construcción de un modelo isótropo y homogéneo}

Para comenzar a construir un modelo de universo a gran escala, es necesario considerar ciertas hipótesis que nos permitan describir las regiones del cosmos que son inaccesibles a la observación astronómica, ya sea por limitaciones tecnológicas, o bien por las restricciones que imponen las leyes de la física. El modelo cosmológico estándar, en particular, se basa en tres pilares fundamentales:

1. El Principio Cosmológico, que establece que, en cada instante, el universo presenta el mismo aspecto en cualquier punto, excepto por las irregularidades locales. Es decir, supone que el universo es espacialmente isótropo y homogéneo.

2. La Teoría de la Relatividad General, como teoría de la gravitación, de la que se desprenden las ecuaciones que permiten estudiar la evolución del universo.

3. El Postulado de Weyl, que establece que las galaxias pueden ser consideradas como partículas fundamentales de un fluido en el espacio-tiempo, y cuyas trayectorias siguen geodésicas ortogonales a hipersuperficies con $t=$ cte, que no se intersecan.

El Principio Cosmológico es una generalización del llamado Principio Copernicano, el cual establece que la Tierra ocupa un lugar de observación "típico" en el universo. Esta condición es menos restrictiva que la hipótesis de homogeneidad, bajo la cual todos los puntos en el universo son equivalentes [1]. El Principio Cosmológico se desprende del Principio Copernicano si se considera además la hipótesis de isotropía a escalas de Hubble. ${ }^{1}$ Es importante resaltar, sin embargo, que la hipótesis de homogeneidad no puede ser testeada mediante observación directa $[1,6]$. Como discutiremos en la sección 1.3, este punto es justamente el que motiva el trabajo que hemos desarrollado en esta tesis. ${ }^{2}$

La Teoría de la Relatividad General está en muy buen acuerdo con numerosos experimentos y observaciones astronómicas [8, 9, 10]. Sin embargo, algunos de los problemas que presenta

\footnotetext{
${ }^{1}$ La hipótesis de isotropía a escalas de Hubble (esto es, a escalas actuales de Gpc, suficientemente grandes como para despreciar las estructuras locales formadas por galaxias), está fuertemente respaldada por las observaciones del fondo cósmico de radiación [2], y la distribución de gamma-ray bursts y otras fuentes distantes [3]. Aún así, algunos modelos homogéneos y anisótropos, como aquellos basados en la solución de Bianchi (apéndice C) presentan características interesantes [4, 5].

${ }^{2}$ Si bien la idea del Principio Cosmológico es simple, sorprende notar que a lo largo de la historia los astrónomos hayan insistido en colocar a la civilización humana en un lugar privilegiado del cosmos (usualmente, el centro). Remontándonos a la antigua Grecia, el modelo geocéntrico desarrollado por C. Ptolomeo (100170) (cuya complejidad para explicar el movimiento de los planetas, la luna y el Sol a partir de epiciclos es realmente extraordinaria) fue aceptado durante más de medio siglo antes de que N. Copérnico (1473-1543) removiera a la Tierra del centro del cosmos para, sin embargo, dejar este lugar privilegiado al Sol. Pasados casi doscientos años, y gracias a la teoría de la gravitación desarrollada por I. Newton (1642-1727) (que enmarcó las las leyes planetarias establecidas empíricamente por J. Kepler (1671-1630)), la configuración del cosmos más aceptada distribuía a las estrellas distantes en un disco alrededor del Sol. Fue hacia finales del 1700 que las observaciones de W. Herschel (1738-1822) asentaron la idea de lo que hoy conocemos como Vía Láctea. Sin embargo, probablemente debido a las limitaciones tecnológicas de la época, el Sol siguió ocupando hasta entonces el centro de esa estructura cósmica. Fue Shapley (1885-1972), a principios del 1900, quien estimó la posición del Sol a una distancia de $2 / 3$ del radio galáctico, reservando, sin embargo, a nuestra galaxia el lugar privilegiado en el centro del universo. Recién en 1952 W. Baade (1893-1960) demostró que la nuestra es una galaxia típica en un universo poblado de este tipo de estructuras celestes, estableciendo así las bases del llamado hoy en día Principio Cosmológico [7].
} 
el modelo cosmológico estándar (los cuales serán discutidos en detalle en la sección 1.2) han impulsado el estudio de teorías alternativas de la gravitación [11]. Entre ellas, nos interesa mencionar a las llamadas teorías de gravedad modificada $f(R)$, basadas en una generalización de la acción de Hilbert-Einstein a funciones más generales del escalar de curvatura $R$ [12, 13]. En forma complementaria al trabajo desarrollado en esta tesis, hemos investigado algunos aspectos de estas teorías, y sus aplicaciones a problemas cosmológicos y astrofísicos. Dichos trabajos están discutidos en el apéndice E.

El Postulado de Weyl, por su parte, enmarca en leyes físicas las observaciones cosmológicas que evidencian que las galaxias, como unidades básicas del fluido cosmológico, se alejan unas de las otras siguiendo un movimiento sistemático. ${ }^{3}$ El postulado propone la existencia de una clase privilegiada de sistemas de referencia en el universo: aquellos independizados del movimiento peculiar de las galaxias. De esta forma, las galaxias son descriptas como partículas fundamentales de un fluido que llena todo el espacio, cuyo movimiento queda determinado por trayectorias geodésicas ortogonales a una familia de hipersuperficies tipo espacio con $t=$ cte. Estas geodésicas no se intersecan, aunque su congruencia pudo haber sido convergente en el pasado y, eventualmente, podría serlo en el futuro. Esto implica que por cada punto del espacio-tiempo pasa una única geodésica y, en consecuencia, es posible definir la velocidad de la materia en forma unívoca [15]. El postulado de Weyl nos permite entonces trabajar con coordenadas comóviles ${ }^{4}\left(t, x^{1}, x^{2}, x^{3}\right)$. Cada hipersuperficie tipo espacio queda definida por un valor $t=$ cte, y a lo largo de cada geodésica las coordenadas $\left(x^{1}, x^{2}, x^{3}\right)$ permanecen constantes (figura 1.1).

La geometría más general que satisface las hipótesis consideradas anteriormente es descripta por la métrica homogénea e isótropa de Friedmann-Lemaitre-Robertson-Walker (FLRW) [18], que viene dada en coordenadas comóviles por el siguiente elemento de línea

$$
\mathrm{d} s^{2}=c^{2} \mathrm{~d} t^{2}-a^{2}(t)\left[\frac{\mathrm{d} r^{2}}{1-k r^{2}}+r^{2}\left(\mathrm{~d} \theta^{2}+\operatorname{sen}^{2} \theta \mathrm{d} \phi\right)\right],
$$

en donde $a(t)$ es el llamado factor de escala, $(r, \theta, \phi)$ son las coordenadas esféricas comóviles, y $t$ es el tiempo propio. Trabajaremos, en adelante, con unidades normalizadas a $c=1$. La constante $k$ es un parámetro asociado a la curvatura espacial; los valores posibles son $k=-1, k=0$ y $k=1$, correspondientes a curvaturas negativa, nula y positiva del 3-espacio, respectivamente. Las ecuaciones de Einstein para el campo gravitacional son

$$
G_{\mu \nu}=8 \pi G T_{\mu \nu}
$$

en donde $G_{\mu \nu}=R_{\mu \nu}-\frac{1}{2} g_{\mu \nu} R$ es el tensor de Einstein, $R_{\mu \nu}=R_{\mu \alpha \nu}^{\alpha}$ es el tensor de Ricci y $R$ es el escalar de curvatura. Trabajaremos, en adelante, con unidades $8 \pi G=1$. El tensor de energía-impulso del fluido perfecto, $T_{\mu \nu}$, caracteriza el contenido de materia en el universo y satisface la ecuación de conservación

$$
\nabla_{\mu} T_{\nu}^{\mu}=0
$$

\footnotetext{
${ }^{3}$ Más precisamente, el Postulado de Weyl surge como solución al problema que plantea cómo aplicar la Teoría de la Relatividad General, basada en la covariancia general entre observadores, a un sistema único como es el universo. Esto es, cómo definir un instante de tiempo particular en Relatividad General si no existen sistemas inerciales globales [14].

${ }^{4}$ Las coordenadas comóviles se definen como aquellas en las que el campo vectorial $u^{\alpha}$ tiene sólo componente temporal no nula, es decir, $u^{\alpha^{\prime}} \propto \delta_{0}^{\alpha^{\prime}}$. Pueden ser introducidas siempre que exista en el espacio-tiempo un campo vectorial tipo tiempo (como lo es, por ejemplo, el 4-vector velocidad de la materia) [16].
} 


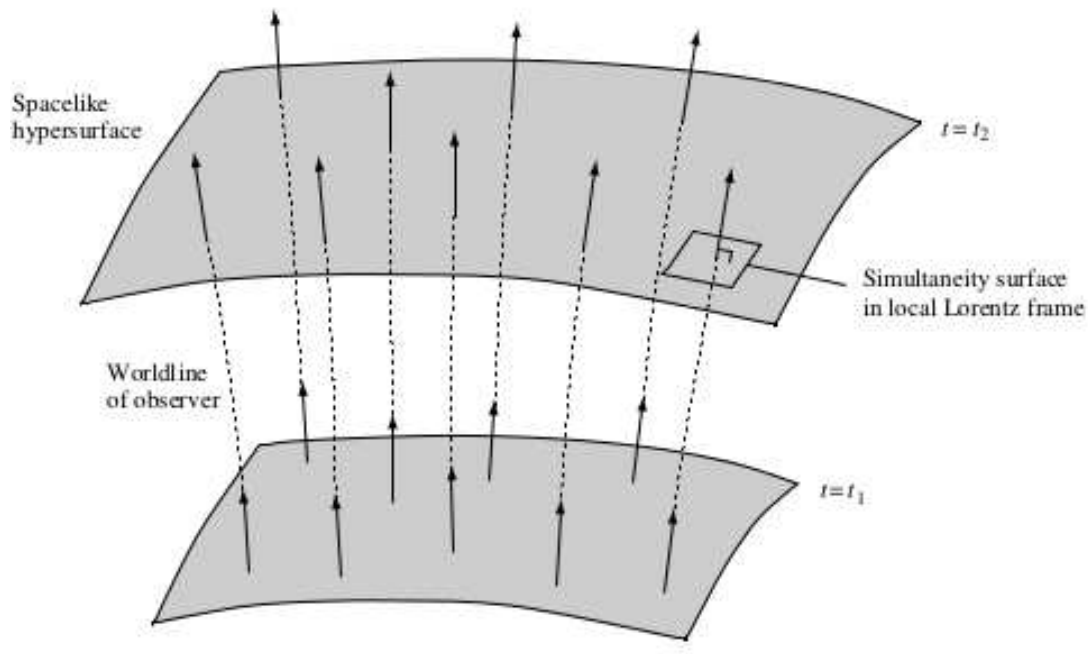

Figura 1.1: (extraída de [17]) Representación del movimiento de las galaxias como partículas fundamentales del fluido cosmológico. Sus trayectorias, en coordenadas comóviles, siguen geodésicas ortogonales a las hipersuperficies con $t=$ cte sin intersecarse.

con

$$
T_{\mu \nu}=\left(\rho+\frac{p}{c^{2}}\right) u_{\mu} u_{\nu}-p g_{\mu \nu}+\Lambda g_{\mu \nu} .
$$

Las ecuaciones de Einstein determinan la dinámica del factor de escala $a(t)$, que dependerá del contenido de materia y energía en el universo. Las ecuaciones de evolución, conocidas como Ecuaciones de Friedmann-Lemaître, resultan [16]

$$
\begin{aligned}
\frac{\ddot{a}}{a} & =-\frac{1}{6}(\rho+3 p)+\frac{1}{3} \Lambda, \\
H^{2}(t) & =\frac{1}{3} \rho+\frac{1}{3} \Lambda-\frac{k}{a^{2}},
\end{aligned}
$$

en donde $H(t) \equiv \dot{a} / a$ es el llamado factor de expansión de Hubble. Por otra parte, de la ecuación de conservación para el fluido, se obtienen la ecuación de continuidad

$$
\nabla_{\mu}\left(\rho u^{\mu}\right)+p \nabla_{\mu} u^{\mu}=0
$$

y la ecuación de movimiento

$$
(\rho+p) u^{\mu} \nabla_{\mu} u^{\nu}=\left(\eta^{\mu \nu}-u^{\mu \nu}\right) \nabla_{\mu} p .
$$

La ecuación (1.8) se satisface idénticamente si las partículas del fluido siguen trayectorias geodésicas. Además, trabajando con la ecuación de continuidad, tenemos que

$$
\dot{\rho}+(\rho+p) \frac{3 \dot{a}}{a}=0 .
$$

Esta última expresión también puede ser obtenida a partir de las ecuaciones de FriedmannLemaître, por lo que sólo dos de las tres ecuaciones (1.5), (1.6) y (1.9) son independientes. 
Para completar el escenario cosmológico, nos resta especificar las componentes de materia en el universo. Según el modelo cosmológico estándar, el universo presenta tres componentes independientes, asociadas a la materia, a la radiación y a la densidad de energía del vacío, respectivamente. Cada componente es modelada por un fluido perfecto con una ecuación de estado de la forma $p_{i}=w_{i} \rho_{i}$. La densidad equivalente total será una función del tiempo, suma de las tres contribuciones individuales. Esto es,

$$
\rho(t)=\rho_{m}(t)+\rho_{r}(t)+\rho_{\Lambda} .
$$

La densidad de materia total incluye la materia ordinaria (partículas bariónicas) y la llamada materia oscura, que se caracteriza por interactuar con la primera casi exclusivamente a través de la gravedad. Una hipótesis adicional es la de considerar que la energía térmica de las partículas de materia es mucho menor que su energía en reposo. Es decir, que podemos modelar a la materia mediante un fluido con presión nula $\left(w_{\mathrm{m}}=0\right)$. La componente de radiación incluye a los fotones, así como también a otras partículas con masa nula o casi nula, con velocidades relativistas, como por ejemplo los neutrinos. La ecuación de estado para la radiación corresponde al valor $w_{\mathrm{r}}=1 / 3$. La densidad de vacío, por su parte, es modelada usualmente con un fluido de densidad constante y parámetro de ecuación de estado $w_{\Lambda}=-1$ (ver sección 1.2.1).

Si suponemos, además, que estas componentes no interactúan entre sí, ${ }^{5}$ y consideramos una evolución en etapas dominadas por cada una de ellas, de la ecuación (1.9) resulta

$$
\rho_{i}(t) \propto a(t)^{-3\left(1+w_{i}\right)} .
$$

Como veremos en la siguiente sección, en el marco del modelo cosmológico estándar la etapa más temprana de la evolución del universo fue dominada por la radiación, para dar luego lugar a las etapas dominadas por la materia y, posteriormente, por la energía oscura.

Las ecuaciones de Friedmann-Lemaître pueden presentarse de una forma adimensional en términos de cantidades normalizadas. Para esto, definimos los llamados parámetros de densidad como

$$
\Omega_{m} \equiv \frac{\kappa \rho_{m}}{3 H^{2}}, \quad \Omega_{r} \equiv \frac{\kappa \rho_{r}}{3 H^{2}}, \quad \Omega_{\Lambda} \equiv \frac{\Lambda}{3 H^{2}}, \quad \Omega_{k} \equiv-\frac{k}{a^{2} H^{2}},
$$

para las componentes de materia, radiación, constante cosmológica y curvatura, respectivamente. ${ }^{6}$ La ecuación (1.6) queda reducida entonces a la forma

$$
\Omega_{m}+\Omega_{r}+\Omega_{\Lambda}+\Omega_{k}=1
$$

Luego, de las ecuaciones de conservación para cada una de las componentes, tenemos

$$
\Omega_{i}(t)=\Omega_{i, 0}\left(\frac{a(t)}{a_{0}}\right)^{-3\left(1+w_{i}\right)}\left(\frac{H_{0}}{H(t)}\right)^{2},
$$

\footnotetext{
${ }^{5}$ Esta hipótesis es válida a partir del momento del desacople entre los fotones y la materia. Una introducción completa sobre la interacción entre los fluidos cosmológicos durante las etapas tempranas de evolución del universo puede encontrarse en la referencia [19].

${ }^{6}$ Las densidades de materia y radiación incluyen distintas especies de partículas con igual ecuación de estado. Esto es, por ejemplo, la materia bariónica y la materia oscura, en el primer caso, y los fotones y los neutrinos, en el segundo. Por simplicidad, continuaremos indicándolas simplemente con las referencias $m$ y $r$.
} 
en donde todas las cantidades señaladas con el subíndice 0 están evaluadas en el tiempo actual. Considerando, además, $a_{0}=1$, resulta

$$
H^{2}=H_{0}^{2}\left[\Omega_{m, 0} a^{-3}+\Omega_{r, 0} a^{-4}+\Omega_{\Lambda, 0}+\Omega_{k, 0} a^{-2}\right] .
$$

La evolución del modelo dependerá de las diferentes contribuciones de los parámetros $\Omega_{i, 0}$.

Por último, las escalas características de tiempo y distancia son expresadas en términos del parámetro de Hubble como

$$
t_{H} \equiv \frac{1}{H}, \quad \text { y } \quad D_{H} \equiv \frac{c}{H} .
$$

El orden de magnitud de estas cantidades puede ser obtenido expresando el parámetro de Hubble medido actualmente en las siguientes unidades

$$
H_{0}=100 h \mathrm{~km} \mathrm{~s}^{-1} \mathrm{Mpc}^{-1} \text {, }
$$

con el valor $h \simeq 0,7$ típicamente adoptado [20].

\subsection{2. $\quad$ El modelo $\Lambda \mathrm{CDM}$}

Habiendo delineado brevemente el marco teórico en el que está basado el modelo estándar, pasaremos a dar una reseña del escenario cosmológico que describe con éxito diferentes conjuntos de observaciones astronómicas, abarcando etapas bien definidas de la evolución cósmica. La bibliografía que abarca este tópico es vasta (ver, por ejemplo, [7, 19, 20, 21, 22]), por lo que nos limitaremos a mencionar aquí sólo los aspectos más generales, profundizando aquellos que tienen más relevancia para los temas desarrollados en esta tesis.

La Teoría de la Relatividad General, junto con observaciones astronómicas de naturaleza diversa, han posibilitado una buena reconstrucción de la historia del universo. La interpretación de estas observaciones en el marco teórico presentado anteriormente, junto con el modelo estándar de las partículas elementales [23], ha dado lugar al desarrollo de un modelo cosmológico estándar que describe con increíble precisión diferentes etapas de la evolución del cosmos.

Según la descripción de dicho modelo, el universo surgió hace aproximadamente 13 mil millones de años de un estado inicial de altísima temperatura y densidad (llamado usualmente big-bang), a partir del cual se ha estado expandiendo en forma continua, dando lugar a un consecuente enfriamiento. Este escenario permite reconstruir la evolución del universo o bien en términos temporales, o bien en términos de las temperaturas que caracterizaron cada una de las etapas de la evolución y los estados que fue atravesando el fluido cosmológico, en lo que conocemos como la historia térmica del universo. ${ }^{7}$ Presentaremos a continuación las características principales de algunas de las etapas más relevantes para nuestro trabajo.

\footnotetext{
${ }^{7}$ En términos estrictos, la solución matemática de FLRW no admite estados de equilibrio térmico (pues no presenta vectores de Killing tipo tiempo). Sin embargo, a fines prácticos es posible aproximar muchas de las etapas de la evolución del universo con diferentes estados de equilibrio térmico. El apartamiento sucesivo de estos estados de equilibrio es justamente lo que dio lugar a la evolución de las estructuras que conocemos hoy en día. La construcción de la historia térmica del universo radica entonces en la comparación sucesiva de las tasas de interacciones entre partículas y de expansión cósmica [24].
} 


\section{Universo muy temprano y época dominada por la radiación}

La reconstrucción de los primeros instantes de la historia cósmica es uno de los mayores desafíos de la cosmología. Para describir la evolución del universo durante la llamada era de Planck (hasta aproximadamente $10^{-43}$ de fracción de segundo después de lo que convencionalmente tomamos como origen del tiempo, cuando la temperatura era del orden de $10^{19}$ $\mathrm{GeV}$ ), es necesaria una teoría de la gravitación que todavía es desconocida. Pasada esta era, a temperaturas $T<m_{p l}$, la gravitación ya puede ser descripta con la Teoría de la Relatividad General. Según el modelo estándar de partículas, a esta temperatura el universo habría estado compuesto de un plasma de partículas relativistas. Entre las teorías de física de partículas que modelan esta etapa del universo, podemos mencionar las Teorías de Gran Unificación (o GUT, por sus siglas en inglés). Las mismas predicen la existencia de una fase de ruptura espontánea de simetrías, con transiciones de fase a temperaturas del orden de $10^{14}$ a $10^{16}$ $\mathrm{GeV}$. Este escenario es el que da lugar a la llamada etapa de inflación, caracterizada por una expansión acelerada del factor de escala del universo [24].

A temperaturas del orden de los $100 \mathrm{MeV}\left(t \sim 10^{-5}\right.$ segundos después del big-bang $)$ el universo habría experimentado una etapa de transición asociada al rompimiento de simetrías quirales y el confinamiento del color. Los bariones y mesones son creados durante esta etapa. ${ }^{8}$ A temperaturas del orden de 10 a $0,1 \mathrm{MeV}\left(t \sim 10^{-2}\right.$ a $10^{2}$ segundos $)$ tiene lugar la etapa de nucleosíntesis primordial [25]. Esta etapa describe las interacciones entre las partículas relativistas en equilibrio, y representa el test del modelo cosmológico estándar más temprano en la evolución del universo. Las evidencias observacionales que la sustentan están basadas en la medición de la abundancia primordial de núcleos livianos [26]. Las mediciones de estos elementos permiten poner cotas sobre el parámetro $\eta \equiv$ número de bariones/número de fotones. ${ }^{9}$

\section{Desacople de los fotones y época dominada por la materia}

La expansión del universo está dominada por la radiación hasta alrededor de 300.000 años después del big-bang. Cuando la temperatura de los fotones desciende hasta $\sim 3.000^{\circ} \mathrm{K}$, tiene lugar la formación de átomos livianos de $\mathrm{H}$ y He a partir de la captura de electrones libres. Los fotones se desacoplan de la materia y dan origen al fondo cósmico de radiación (o CMB, por sus siglas en inglés) [29].

El fondo cósmico de radiación representa una de las evidencias observacionales más fuertes del modelo cosmológico estándar [2]. La radiación medida ajusta con alta precisión la emisión de un cuerpo negro con una temperatura efectiva de $2,75^{\circ} \mathrm{K}$, y presenta una distribución isótropa en escalas angulares del orden de $10^{-5}$. Los mapas de anisotropías en la temperatura de los fotones proveen información tanto del momento del desacople (e.g. sobre el espectro primordial de las perturbaciones en la densidad), como de los efectos sufridos por los fotones a lo largo de su trayectoria desde la superficie de último scattering hasta el momento actual (como, por ejemplo, el efecto Sachs-Wolfe integrado [30] y el efecto Sunyaev-Zel'dovich cinemático [31], entre otros). Otra fuerte evidencia observacional del momento de pre-desacople son las oscilaciones acústicas de bariones (BAO), utilizadas como reglas patrón [32].

\footnotetext{
${ }^{8}$ Sin entrar en los detalles del modelo estándar de partículas, cabe mencionar que el problema de modelar regiones locales no homogéneas en la densidad del número de bariones durante esta etapa puede ser abordado también con el modelo no homogéneo de dos fluidos que presentaremos en el capítulo 5.

${ }^{9} \mathrm{La}$ abundancia del ${ }^{7} \mathrm{Li}$ representa, sin embargo, un tema de discusión abierta, debido a las serias discrepancias entre el valor medido y el valor estimado por el modelo [27, 28].
} 
La formación de estructuras a gran escala en el universo es una consecuencia de la amplificación gravitacional de pequeñas fluctuaciones en la densidad de materia sin presión y que no interactúa electromagnéticamente (esto es, la llamada materia oscura fría o Cold Dark Matter (CDM) ) [33, 34, 35]. Las evidencias observacionales que permiten medir el espectro de potencias de la distribución de materia están basadas en la construcción de grandes catálogos de galaxias. Entre aquellos sondeos con más cantidad de objetos y una cobertura amplia del cielo, podemos mencionar el Sloan Digital Sky Survey (SDSS) ${ }^{10}$ y el 2-degree field Galaxy Redshift Survey (2dfGRs) ${ }^{11}$, que proporcionan una evidencia de la estructura cósmica hasta distancias de $z \sim 0,3 .^{12}$

\section{Expansión acelerada y época dominada por la energía oscura}

La expansión del universo está fuertemente justificada por diversos conjuntos de datos y constituye uno de los pilares fundamentales en la construcción del modelo cosmológico. La primera evidencia observacional de la expansión del universo fue presentada por E. Hubble en 1929 [37] a partir de la medición independiente de las distancias y velocidades de recesión de galaxias cercanas. Hubble notó que la curva distancia vs. velocidad seguía la relación lineal empírica $d \sim \frac{v}{H_{0}}$, de donde podía inferirse directamente el valor de la constante $H_{0}$.

Hoy en día, gracias a la innovación de métodos para construir escalas de distancias ${ }^{13}$ y, fundamentalmente, al salto observacional que ha conquistado la astronomía a través del Telescopio Espacial Hubble, es posible medir la constante de Hubble en $H_{0} \sim 70 \mathrm{~km} / \mathrm{s} / \mathrm{Mpc}$ con una precisión aproximada del $10 \%$ [39]. Estos resultados, por otra parte, están en buen acuerdo con la medición de $H_{0}$ a partir de otros métodos que no requieren la calibración de una escala de distancias y envuelven procesos físicos diversos. ${ }^{14}$

Entre los métodos utilizados para construir escalas de distancia, la relación distancia de luminosidad vs. redshift de las SN tipo Ia presenta algunas ventajas que vale la pena destacar. ${ }^{15}$ Las explosiones de SN tipo Ia tienen su origen en sistemas binarios con estrellas enanas blancas acretantes. La curva de luminosidad puede ser calibrada usando una relación empírica bien establecida entre la posición del máximo y el ancho de la curva, razón por la cual estos eventos pueden ser utilizados como "velas patrón" en la medición de distancias. Por otra parte, dado que la luminosidad máxima involucrada en una explosión de SN tipo Ia alcanza

\footnotetext{
${ }^{10}$ http://www.sdss.org/

${ }^{11}$ http://www.2dfgrs.net/

${ }^{12}$ Es importante mencionar que la relación entre este tipo de observaciones y el espectro de potencias tridimensional de la materia oscura no es directo, dado que sólo la materia bariónica puede ser observada. Para derivar conclusiones relevantes sobre la distribución total de materia es entonces necesario trabajar bajo la hipótesis de que la materia luminosa es efectivamente un buen trazador de materia oscura [36].

${ }^{13}$ Entre estos métodos, podemos mencionar [20,38] (i) la calibración de la relación período de pulsación vs. luminosidad de las variables Cefeidas; (ii) la curva de luminosidad de las SN tipo Ia; (iii) la relación Tully-Fisher entre la luminosidad total y la velocidad de rotación máxima de galaxias espirales; (iv) la correlación entre la luminosidad intrínseca de galaxias elípticas y la dispersión de velocidades de las estrellas que las componen; y (v) las fluctuaciones de brillo superficial de galaxias observadas en cámaras CCD $(z<0,02)$.

${ }^{14}$ Estos métodos incluyen [20] (i) la medición del radio de la fotósfera de SN tipo II [40]; (ii) mediciones del retardo entre dos señales provenientes de imágenes de lente gravitacional de quásares variables [41, 42]; y (iii) el efecto Sunyaev-Zel'dovich térmico [43] inducido en fotones del fondo cósmico de radiación [44, 45].

${ }^{15} \mathrm{El}$ redshift cosmológico es una medida del corrimiento de líneas espectrales de emisión hacia longitudes de onda mayores debido a la expansión cósmica. La mayoría de las observaciones astronómicas independientes de cualquier modelo cosmológico proveen la posición angular en la esfera celeste y el redshift de la fuente. La distancia radial no es accesible a medición directa sin involucrar un modelo implícito para la expansión del universo.
} 
valores comparables a la luminosidad total de la galaxia que las contiene, estos eventos pueden ser observados a distancias de varios cientos de Mpcs. Es por estas razones que la curva de luminosidad de las SN tipo Ia es utilizada, no sólo para la estimación de la constante de Hubble a grandes redshifts, sino también para la determinación de otros parámetros cosmológicos.

En 1998, dos grupos de investigación independientes (el Supernova Cosmology Project (SCP) [46] y el High-Z Supernova Search Team (HZT) [47]), abocados al estudio de SN tipo Ia distantes, publicaron los resultados que harían cambiar radicalmente la descripción actual del cosmos. Ambos grupos mostraron que el ajuste de los parámetros cosmológicos daba lugar a un valor negativo para el llamado parámetro de desaceleración, $q_{0} \equiv-\left(\frac{\ddot{a}}{a H^{2}}\right)_{t_{0}}$. Es decir que, contrariamente al comportamiento esperable de una dinámica dominada por materia, el universo se encontraría en una etapa de expansión acelerada [48, 49, 50, 51, 52]. La consecuencia directa que se desprende del análisis de estos resultados, interpretados en el marco del modelo estándar, es que la constante cosmológica debe tener un valor positivo no nulo.

Actualmente, la curva de luminosidad de las SN tipo Ia, sumada a otros conjuntos de observaciones (como el proveniente del fondo cósmico de radiación), restringen el espacio de parámetros del modelo cosmológico estándar en forma consistente. ${ }^{16}$ Los valores inferidos para los parámetros cosmológicos modelan un universo con curvatura prácticamente nula $\left(\Omega_{k} \simeq 0\right.$ ), y parámetros de densidad dados por $\Omega_{\Lambda, 0} \simeq 0,7, \Omega_{m, 0} \simeq 0,3, \Omega_{r, 0} \simeq 10^{-4}$ y $\Omega_{k, 0} \simeq 0$. Es decir, que la dinámica actual queda dominada por $\Omega_{\Lambda}$, con una evolución del factor de escala del tipo $a(t) \sim \mathrm{e}^{t \Lambda / 3}$ (esto es, expansión acelerada). El modelo que describe esta dinámica es usualmente llamado modelo $\Lambda \mathrm{CDM}$, en alusión a las componentes actualmente dominantes (constante cosmológica, $\Lambda$, y la materia oscura fría, $\mathrm{CDM}$ ).

\subsection{Problemas del modelo cosmológico estándar}

Vimos hasta aquí que el modelo cosmológico estándar está construido bajo hipótesis simples y ofrece una descripción precisa de diferentes etapas de la evolución del universo, cada una fuertemente justificada con argumentos teóricos sólidos, y respaldada por conjuntos de observaciones de naturaleza diversa (ver figura 1.2). Sin embargo, el modelo presenta algunos problemas para los cuales existen hasta el momento sólo soluciones parciales. Las posibles direcciones que pueden tomarse para abordar cada uno de ellos están fuertemente relacionadas con las motivaciones del trabajo desarrollado en esta tesis.

\footnotetext{
${ }^{16}$ Una restricción adicional sobre los parámetros cosmológicos del modelo puede obtenerse a partir de la estimación de la edad de diferentes objetos celestes a través de observaciones cosmológicas. Esto es, imponiendo una cota mínima para la edad dinámica del universo calculada a partir de las soluciones de las ecuaciones de Friedmann-Lemaître. La edad dinámica calculada a partir de los parámetros ajustados con SN tipo Ia resulta $t_{0} \sim 14 \times 10^{9}$ años. Por otra parte, la edad de la Vía Láctea puede estimarse a partir de estrellas de baja metalicidad, distribuidas espacialemnte en el halo de nuestra galaxia. Los métodos para determinar sus edades son: (i) la nucleocronología, para estrellas con abundancias de torio o uranio [53, 54]; (ii) el enfriamiento de estrellas enanas blancas observadas en la vecindad del sistema solar ; y (iii) la edad de cúmulos globulares galácticos a partir del cut-off de su secuencia principal. Todos estos métodos arrojan cotas entre 11 mil y 15 mil millones de años $[45,55,56,57]$. Estas cotas, sin embargo, dependen del modelo utilizado para estimar el comienzo de la formación de estrellas. Suponiendo que la formación estelar tuvo su comienzo en tiempos correspondientes a $z$ entre 5 y 20 (que en el modelo $\Lambda$ CDM corresponde a tiempos entre 0,1 y $2 \times 10^{9}$ años), las cotas para la edad del universo resultan $9 \times 10^{9}$ años $<t_{0}<15 \times 10^{9}$ años.
} 


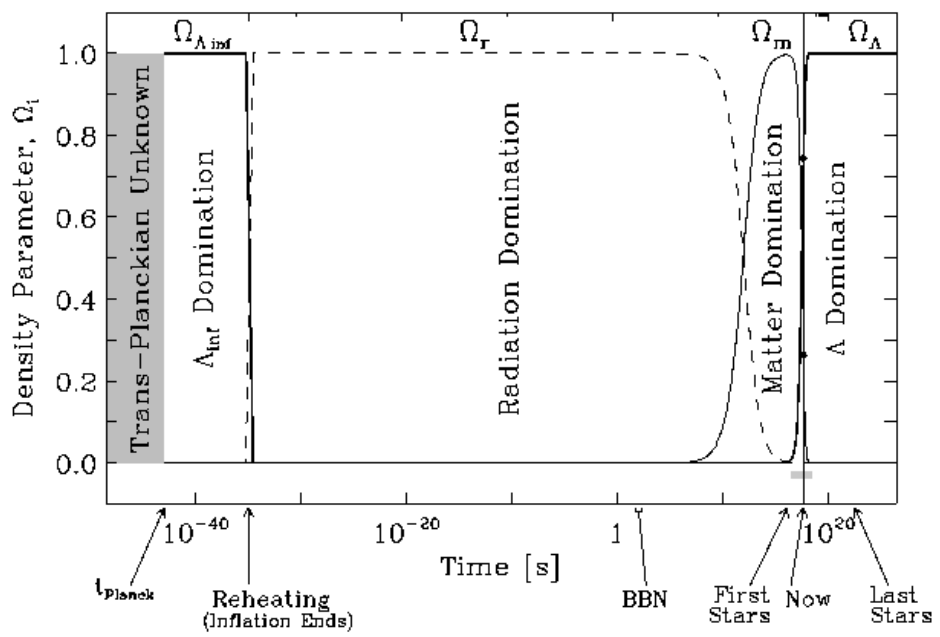

Figura 1.2: (extraída de [58]) Esquema representativo de la historia del universo, en términos de la componente de fluido dominante en cada etapa de la evolución.

\section{El problema del horizonte}

Como vimos en la sección 1.1.1, una de las características del modelo estándar es que el factor de escala $a(t)$ crece como $t^{n}$, con $n<1$. Una consecuencia directa de esta dinámica es la aparición de un horizonte de partículas [59], que lleva a que las líneas de universo de partículas provenientes de regiones no conectadas de forma causal nunca lleguen a intersecarse. Si reconstruimos su historia a partir de los elementos del modelo presentados hasta ahora, encontramos que el universo observable presenta actualmente alrededor de $10^{5}$ regiones que debieron estar desconectadas en forma causal en la época de recombinación, cuando los fotones del fondo cósmico de radiación atravesaron la etapa de último scattering. Sin embargo, las evidencias observacionales obtenidas por los satélites WMAP y Planck muestran uniformidad en los mapas de anisotropías del fondo cósmico de radiación de aproximadamente una parte en $10^{-5}$ [2]. En consecuencia, sin ingredientes adicionales al modelo, las hipótesis de homogeneidad e isotropía a grandes escalas no podrían ser justificadas mediante procesos causales $[59,60,61]$.

\section{El problema de la curvatura plana}

El llamado problema de la curvatura plana (o flatness problem) está asociado al valor nulo que inferimos para el parámetro de curvatura, y puede entenderse como una competencia de términos en la ecuación (1.6) para la evolución del factor de escala.

Para ver esto, definamos la densidad crítica $\rho_{c}$ como aquella densidad para la cual el universo tiene exactamente curvatura nula. Esto es,

$$
\rho_{c}=3 H^{2}=3 \frac{\dot{a}^{2}}{a^{2}} .
$$

El cociente $\left|\rho-\rho_{c}\right| / \rho_{c}$ es entonces una medida de cuán cerca está el universo de ser descripto por una solución de FLRW con $k=0$. Si extrapolamos la ecuación (1.6) a tiempos tempranos de la evolución del universo $(t \rightarrow 0)$, y tenemos en cuenta que en esta época la dinámica 
estuvo dominada por la radiación, $\rho_{r} \sim a^{-4}$, resulta que cerca de la época de Planck debió tomar valores tales que

$$
\frac{\left|\rho-\rho_{c}\right|}{\rho_{c}}<10^{-59}
$$

para alcanzar un radio actual dado por $H_{0}^{-1}\left(10^{28} \mathrm{~cm}\right)$.

Es decir, que el valor de la densidad en tiempos tempranos tuvo que ser extremadamente cercano al valor de la densidad crítica. Un mínimo apartamiento de esta cota hubiera dado lugar a una evolución completamente diferente de la que el modelo describe. El problema radica entonces en poder explicar esta condición inicial sumamente especial para que el universo, descripto dentro del modelo estándar, haya evolucionado al estado que observamos hoy en día.

\section{El problema de las reliquias o monopolos primordiales}

De acuerdo a las GUT que enmarcan el escenario del universo primordial, las transiciones de fase que tuvieron lugar durante las épocas más tempranas de la evolución debieron haber producido monopolos magnéticos, esto es, partículas supermasivas $\left(\sim 10^{16}\right.$ veces más masivas que un protón) con una sección eficaz de aniquilación muy pequeña [62]. De acuerdo al modelo estándar de partículas, estos monopolos deberían haber sobrevivido hasta el presente, con una densidad actual del mismo orden que la densidad de fotones. Como consecuencia, la densidad crítica del universo resultaría $10^{15}$ veces mayor que la observada hoy en día $\left(\rho_{c} \sim 10^{-29} \mathrm{~g} \mathrm{~cm}^{-3}\right)$ [63]. Esta densidad excesiva en las etapas tempranas de la evolución del universo debería haber dominado la dinámica, dando lugar a consecuencias catastróficas [20]. Esto es lo que conocemos como problema de los monopolos primordiales [64, 65, 66].

Es entonces necesario generar algún mecanismo complementario al modelo cosmológico capaz de diluir la densidad de los monopolos primordiales. Cabe destacar, sin embargo, que existen aún incertezas sobre la sección eficaz de aniquilación de los monopolos y, en consecuencia, este problema podría corresponder al dominio de la teoría de campos [67].

\section{El problema del origen de las fluctuaciones primordiales}

Para explicar la formación de estructuras a escalas galácticas en un modelo homogéneo, es necesario pproponer algún mecanismo que dé lugar a pequeñas perturbaciones en la distribución primordial de densidad luego del momento del desacople entre la radiación y la materia $\left(z \sim 10^{4}\right)$.

El tamaño de estas sobredensidades, de origen térmico está acotado por las anisotropías observadas hoy en el fondo cósmico de radiación [2]. Dado un espectro primordial para las sobredensidades, la formación de estructuras puede ser estudiada entonces a partir de un formalismo de perturbaciones en el modelo homogéneo descripto por la solución de FLRW.

El llamado problema del origen de las fluctuaciones radica entonces en encontrar algún mecanismo que haya dado lugar al espectro primordial de perturbaciones en forma general (esto es, sin que las fluctuaciones tengan que ser elegidas arbitrariamente).

\subsubsection{Energía oscura}

El descubrimiento de la expansión acelerada del universo ha cambiado radicalmente nuestra descripción del cosmos. Dentro de la evidencia observacional que sustenta dicha aceleración 
podemos destacar: (i) la compatibilidad entre la edad de los objetos más viejos y la edad del universo [68, 69, 70, 71]; (ii) la curva de distancia de luminosidad vs. redshift observada para las SN tipo Ia [46, 47] y los gamma-ray bursts [72, 73], utilizados como velas patrón; y (iii) las anisotropías en el CMB observadas por los satélites COBE, WMAP y Planck [2].

Los mencionados conjuntos de datos provienen de procesos físicos de origen diverso (que tuvieron lugar en momentos bien diferentes de la evolución del universo y con escalas de tiempo dispares); interpretados según el modelo $\Lambda \mathrm{CDM}$, indican que el universo estaría dominado hoy en día por la llamada "energía oscura", en una proporción de aproximadamente $7 / 3$ con relación a la materia. Cabe resaltar que para ser la fuente de la expansión acelerada, esta nueva forma de materia debe tener características peculiares: presión negativa (esto es, tensión) y distribución homogénea [74]. ${ }^{17}$

Existen varios candidatos para describir la energía oscura dentro del modelo cosmológico estándar. Entre ellos, podemos mencionar: ${ }^{18}$

1. la constante cosmológica;

2. la quintaesencia (y otros modelos que utilizan campos escalares); y

3. las teorías $f(R)$ de la gravitación, que difieren de la Relatividad General en determinados regímenes de curvatura.

A pesar de haber recibido bastante atención en la literatura [71], estos candidatos presentan problemas serios. La constante cosmológica es compatible con los datos observacionales obtenidos hasta hoy, pero el valor inferido de las observaciones difiere de aquel predicho por la teoría cuántica de campos en, por lo menos, 30 órdenes de magnitud [74].

La quintaesencia y los modelos afines tienen la ventaja de poder modelar una eventual evolución de la energía oscura con el redshift, pero utilizan como elemento básico un campo escalar clásico (entidad interesante desde el punto de vista teórico, pero que todavía no ha sido observada en la naturaleza), que responde a un potencial con características peculiares dentro de la física de partículas [71].

Por último, las teorías $f(R)$ de la gravitación $[12,13,78]$ son una generalización natural de la Relatividad General, y permiten describir una energía oscura variable con $z$. El punto débil de este abordaje es la indeterminación grande que tenemos sobre las funciones $f(R)$ [79], a pesar de las restricciones impuestas para evitar inestabilidades en objetos compactos y satisfacer vínculos provenientes de observaciones en el Sistema Solar y de experimentos en laboratorios [80, 81, 82].

Este escenario oscuro nos invita a abandonar las propuestas anteriores, y a revisar seriamente las hipótesis en las que basamos nuestra descripción del cosmos. Entre ellas, la que representa uno de los pilares fundamentales del modelo cosmológico estándar es el Principio

\footnotetext{
${ }^{17}$ Como está señalado en [74], el término "energía oscura" no es, estrictamente hablando, el nombre más apropiado para describir a esta sustancia (pues muchas otras cosas en la naturaleza son oscuras y poseen energía). Lo que distingue a la energía oscura de la materia ordinaria es su presión negativa (esto es, tensión), que da lugar a la aceleración del universo. Luego, "tensión oscura" parece ser un término más apropiado, incluso mejor que "presión oscura". Dado que, además, su distribución espacial es aparentemente suave o uniforme, y no está concentrada en pozos de potencial, una forma más adecuada para designar a la "energía oscura" sería "tensión regular" o "tensión suave".

${ }^{18}$ Existen modelos (más) exóticos, como por ejemplo el electromagnetismo no lineal [75], el gas de Chaplygin [76], y los mundos-brana [77].
} 


\section{Cosmológico. $^{19}$}

Como fue señalado anteriormente, las hipótesis de homogeneidad e isotropía en el modelo modelo estándar son independientes. Si bien la isotropía a grandes escalas tiene un buen sustento observacional (sondeos de fuentes de radio e infrarrojas, y el CMB [86]), la hipótesis de homogeneidad, que está basada en el Principio Copernicano, no es una consecuencia directa de las observaciones (a pesar de ser consistente con ellas mediante la introducción de materia y energía "oscuras"). En este sentido, y sin poner en discusión su carácter filosófico, el Principio Cosmológico debe ser estudiado con cuidado antes de descartar la posibilidad de describir el universo con modelos no homogéneos. De hecho, no es trivial que pueda utilizarse un modelo altamente simétrico para interpretar datos que involucran fenómenos físicos en regiones sin simetrías predominantes, y en las que el mayor porcentaje de la masa está formando parte de estructuras. Un test de homogeneidad serio requeriría considerar todos los puntos del espaciotiempo, y mostrar que en cada uno de estos las observaciones cosmológicas se ajustan a un escenario isótropo. ${ }^{20}$

Para ser entonces consistentes con el método científico, debemos someter la hipótesis de homogeneidad al test de la observación [88, 89, 90, 91]. Una forma de llevar a cabo esta tarea es a través de la utilización de modelos cosmológicos no homogéneos, construidos a partir de soluciones exactas no homogéneas de las ecuaciones de Einstein. Esta es la principal motivación del trabajo desarrollado en esta tesis. ${ }^{21}$

\subsubsection{Inflación}

La llamada etapa de inflación surge como un ingrediente fundamental en el modelo cosmológico estándar para dar una solución a algunos de los problemas mencionados al comienzo de esta sección [63]. La misma describe un período de expansión acelerada durante la evolución más temprana del universo $\left(t \sim 10^{-43}\right.$ segundos $)$, en donde el factor de escala evoluciona de la forma $a(t) \sim \mathrm{e}^{\alpha t}$, con $\alpha \simeq$ cte. $^{22}$

Si bien es aceptado actualmente que la existencia de esta etapa de expansión exponencial puede remover de manera eficiente algunas dificultades del modelo comsológico estándar, los modelos inflacionarios por sí mismos no están exentos de presentar aspectos problemáticos. En particular, una de las hipótesis fundamentales en la que dichos modelos se basan es la de suponer un espacio-tiempo homogéneo e isótropo como condición inicial para que el proceso de inflación tenga lugar. La justificación para trabajar con esta elección particular es

\footnotetext{
${ }^{19}$ De hecho, las modificaciones que son necesarias incluir en el modelo estándar para describir las observaciones (esto es, la adición de un "mundo oscuro", compuesto de materia [83] y energía oscura, que transforman a la gravitación de atractiva en repulsiva) son fuertes reminiscencias de la vetusta metodología que llevó a postular los epiciclos ptolemaicos, o un éter para la propagación electromagnética [84]. La propuesta de una constante cosmológica (o densidad de vacío), por su parte, ha sido invocada y abandonada en reiteradas ocasiones en la historia de la cosmología para describir, curiosamente, escenarios de naturaleza diferente (desde A. Einstein, para modelar un universo estático, hasta el modelo cosmológico actual, para dar lugar a una expansión acelerada del universo) [85].

${ }^{20}$ Una discusión interesante sobre este punto, y sobre la posibilidad de construir modelos no homogéneos que no violen el Principio Cosmológico (a partir de observadores que no siguen trayectorias geodésicas), puede encontrarse en la referencia [87].

${ }^{21}$ Existen otros tratamientos para estudiar regiones no homogéneas que no utilizan soluciones exactas de las ecuaciones de Einstein, como aquel basado en las medias [92]. Sin embargo, ciertas dificultades conceptuales restringen su utilidad y, por tanto, no serán tenidos en cuenta en nuestro trabajo [93].

${ }^{22}$ Como detallaremos en el capítulo 4, el valor de la constante $\alpha$ está asociado al potencial $V(\phi)$ de un campo escalar masivo $\phi$.
} 
que, de haber existido regiones no homogéneas previas a la etapa inflacionaria, éstas deberían haber sido diluidas durante el propio proceso de expansión exponencial. Dado que no hay hasta el momento una prueba definitiva de que la inflación tenga efectivamente la propiedad de desarrollarse ante cualquier escenario primordial, es importante abordar este análisis minusciosamente.

En esta dirección, es posible entonces utilizar soluciones no homogéneas de las ecuaciones de Einstein para modelar escenarios de inflación y estudiar condiciones generales bajo las cuales los procesos inflacionarios en escenarios no homogéneos pueden o no tener lugar.

\subsection{Modelos cosmológicos no homogéneos}

De las hipótesis sobre las que está basado el modelo cosmológico estándar, la de homogeneidad a grandes escalas (Gpcs) es acaso una de las más fuertes y difíciles de cotejar con evidencias observacionales directas. Esta dificultad radica en el simple hecho de que estamos situados, como observadores, en un único punto del espacio, así como también en la difícil tarea que implica discernir, en las observaciones astronómicas, evoluciones temporales de variaciones espaciales.

Es posible, sin embargo, considerar diferentes estrategias para abordar tests de homogeneidad. Entre estas, destacamos [6]:

1. La construcción de modelos no homogéneos. Esta posibilidad ha sido explorada con creciente interés en los últimos años. Los modelos basados en soluciones de la Relatividad General más generales pueden presentar rasgos distintivos que permitan diferenciarlos de los modelos de FLRW mediante la observación [94, 95]. Estos modelos, sin embargo, representan más una alternativa a modelos con energía oscura que un test estricto de homogeneidad.

2. Los tests de consistencia. Los modelos de FLRW pueden ser formulados como una serie de hipótesis observacionales nulas, esto es, cantidades que deben ser idénticamente nulas si el modelo responde a la geometría de FLRW y a las relaciones observacionales que de ella se desprenden. La idea de los tests de consistencia es entonces combinar diferentes observables que puedan revelar cualquier señal no nula, como medida de cuánto el modelo se aparta de la hipótesis de homogeneidad. Idealmente estos tests son independientes del modelo de energía oscura o de la teoría de gravedad utilizados. Sin embargo, ofrecen un entendimiento del Principio Copernicano basado en refutar las hipótesis observacionales nulas más que probarlas. ${ }^{23}$

3. La reconstrucción del modelo como un problema inverso. En lugar de postular un modelo cosmológico desde tiempos tempranos (basado en la métrica de FLRW más un tratamiento de perturbaciones) y comparar su evolución con observaciones actuales, este método propone construir un modelo considerando dichas observaciones como "condiciones iniciales" para determinar la métrica sobre el cono de luz pasado del observador, y luego integrar hacia el interior de este [96, 97]. Esta manera de reconstruir el modelo

\footnotetext{
${ }^{23}$ Por ejemplo, la relación distancia de luminosidad vs. redshift de las SN tipo Ia es reconstruida a partir de datos observacionales evitando introducir una dependencia con el modelo. Sin embargo, la aplicación robusta de estos datos necesita en general la incorporación de un modelo cosmológico, al igual que las BAO para ser utilizadas como reglas patrón [6].
} 
cosmológico no necesita en absoluto suponer a priori la validez del Principio Copernicano. Idealmente, a partir de observaciones perfectas sería posible obtener la información necesaria para reconstruir el modelo de universo correcto dentro de nuestro cono de luz pasado. Sin embargo, la eficacia del método demanda observaciones tan precisas que resulta, por el momento, impracticable.

El trabajo desarrollado en esta tesis se encuentra dentro del primer enfoque. Como fue señalado originalmente en la referencia [98], las soluciones de las ecuaciones de Einstein que describen modelos cosmológicos homogéneos e isótropos limitan la aplicación de la Teoría de la Relatividad General a la descripción de nuestro universo, principalmente debido al alto grado de simetría que las caracteriza. En este sentido, las soluciones no homogéneas han sido en general poco exploradas, a pesar de su potencial para describir escenarios complejos con menos simetrías. ${ }^{24}$

Comenzando por la primera solución no homogénea de las ecuaciones de Einstein, encontrada por Lemaître en 1933 [106], y posteriormente analizada por Tolman en 1934 [107] y Bondi en 1947 [98], existe una amplia bibliografía sobre modelos cosmológicos no homogéneos $[16,108,109]$. En particular, nos abocaremos a estudiar en esta tesis aquellos modelos basados en soluciones exactas con simetría esférica. Los modelos más conocidos y estudiados de esta clase son los de Lemaître-Tolman-Bondi (LTB) [98, 106, 107], pero existen otros aún no explotados totalmente en astronomía y cosmología, tales como aquellos basados en la solución de Lemaître [106], en la de Székeres [110, 111] y en la de Stephani [108], entre otros.

La métrica asociada el modelo de LTB es una solución de las ecuaciones de Einstein con simetría esférica y con polvo como fuente. La misma depende de dos funciones arbitrarias, y permite estudiar efectos no lineales en la evolución de forma analítica y numérica [16, 108, 109]. La solución de LTB ha sido ampliamente estudiada con el fin de construir modelos cosmológicos viables para poder describir diferentes conjuntos de observaciones astronómicas sin la necesidad de recurrir a un contenido de energía oscura en el universo [109, 112, 113, 114, 115, 116, 117, 118, 119, 120]. Entre los trabajos dedicados a estudiar cosmologías de LTB, los aspectos geométricos más importantes han sido analizados en [121, 122], mientras que las comparaciones con datos observacionales más relevantes pueden encontrarse en las referencias $[123,124,125,126,127,128]$.

Entre los modelos de LTB más destacados, nos interesa mencionar los llamados modelos de void, caracterizados por presentar un perfil inicial no homogéneo con una región de subdensidad en el centro de simetría, inmersa en una distribución de materia asintóticamente homogénea. Para lograr un buen acuerdo de este tipo de modelos con los datos provenientes del fondo cósmico de radiación, es necesario restringir los puntos de observación al centro de simetría del void (o, cuanto mucho, a una distancia de algunas decenas de Mpc [129, 130, 131, 132]). Este aspecto representa uno de los principales puntos conflictivos de los modelos de void. Sin embargo, como está mencionado en [133], la baja probabilidad de estar ubicados como observadores en esta posición especial es mucho menos restrictiva que la coincidencia observada en el valor actual de la constante cosmológica del el modelo estándar. Si pensamos en la probabilidad de que un observador esté ubicado dentro de algunas decenas de Mpc del centro

\footnotetext{
${ }^{24}$ Un modelo no homogéneo, pero construido a partir de soluciones de FLRW, fue originalmente propuesto por K. Tomita en las referencias [99, 100, 101, 102, 103, 104, 105]. El problema consistía en dos regiones homogéneas, separadas por una superficie esférica, expandiéndose cada una con diferente parámetro de Hubble. Con este escenario simple se mostró que es posible reinterpretar las observaciones astronómicas, incluso utilizando soluciones homogéneas, sin recurrir a un contenido de energía oscura.
} 
de una esfera de radio $\sim 15 \mathrm{Gpc}$, resulta $(40 \mathrm{Mpc} / 15 \mathrm{Gpc})^{3} \sim 10^{-8}$. La coincidencia es entonces en este caso considerablemente mucho menor que aquella involucrada, por ejemplo, en el problema de la curvatura espacial [133]. En este sentido, es absolutamente relevante llevar a cabo un estudio minucioso de los modelos de void, como un primer paso en la construcción de escenarios cosmológicos más complejos.

La solución no homogénea de Lemaître [106] presenta la misma simetría que la de LTB, pero contempla la posibilidad de modelar fluidos con presión no nula. A diferencia de la solución de LTB, la de Lemaître ha sido poco utilizada hasta el momento para construir modelos no homogéneos que lleven en cuenta los efectos de una distribución no homogénea de materia con presión. El estudio de las soluciones que describen un fluido con presión es sumamente importante, ya que la misma puede tener efectos significativos sobre la propagación de los fotones en geometrías no homogéneas [134]. En particular, la presión de la radiación proporciona un grado de libertad extra en el problema, que permitiría aliviar algunas tensiones existentes entre los modelos no homogéneos con simetría esférica y ciertas observaciones astronómicas [133]. La investigación de modelos que contemplen estas características merece entonces ser llevada a cabo de manera más profunda.

En todos los casos con simetría esférica, los puntos de observación fuera del centro de simetría han sido muy poco estudiados, en general, por la complejidad matemática que caracteriza a estas geometrías [128]. Soluciones con menos simetría, como la de Stephani y Székeres, permiten modelar regiones no homogéneas más generales. De la clase de soluciones encontrada por Stephani [135], la más estudiada es la que tiene simetría esférica, con densidad dependiente de la coordenada radial, y presión dependiente de las coordenadas $r$ y $t[136] .{ }^{25}$ La métrica de Székeres, por su parte, tiene 5 funciones arbitrarias, con polvo como fuente, y no posee vectores de Killing [108]. Existen 3 tipos de soluciones de Székeres (cuasiesférica, cuasiplana, y cuasipseudoesférica), de los cuales solamente el primero ha sido aplicado en cosmología y astronomía [137]. La solución de Szafron [138], por su parte, generaliza a la de Székeres con la incorporación de la presión.

\subsection{Objetivos de esta tesis}

El objetivo general de esta tesis es investigar modelos cosmológicos en el marco de la Teoría de la Relatividad General, que sean compatibles con las observaciones astronómicas y que no precisen de la llamada "energía oscura" como componente de materia dominante en la dinámica actual del universo. El trabajo que desarrollamos distingue dos etapas principales:

1. el estudio de las propiedades genéricas de soluciones exactas no homogéneas de las ecuaciones de Einstein; y

2. la aplicación de dichas soluciones a problemas de interés cosmológico.

En relación a la primera etapa, nos abocaremos a estudiar, en particular, aquellas soluciones que presentan simetría esférica. En orden creciente de complejidad, analizaremos primero la solución de Lemaître-Tolman-Bondi (LTB), que describe un fluido con distribución de materia no homogénea y sin presión (es decir, polvo). La segunda solución estudiada presentada

\footnotetext{
${ }^{25}$ Un análisis de los modelos cosmológicos basados en la solución de Stephani, que incluye una discusión interesante sobre la posibilidad de estudiar modelos no homogéneos que satisfagan el Principio Copernicano en un sentido observacional (esto es, a partir de relaciones observacionales válidas para cualquier punto del espacio-tiempo) puede encontrarse en [87].
} 
será la de Lemaître, que contempla la descripción de un fluido no homogéneo y con presión. Las propiedades genéricas de estas soluciones serán presentadas en el capítulo 2, junto con una introducción al campo de aplicación de cada una para resolver diferentes problemas de cosmología y astrofísica. Como mencionamos en la sección anterior, las soluciones con simetría esférica representan el primer paso para la construcción de modelos más complejos. En esta dirección, algunas propiedades de la solución de Székeres también serán investigadas en forma complementaria al trabajo principal de la tesis.

En relación a la segunda etapa del trabajo, las soluciones antes mencionadas serán aplicadas para resolver diferentes problemas cosmológicos, a saber:

1. El estudio de la variación temporal del corrimiento al rojo cosmológico debida a la tasa variable de expansión del universo.

Esta cantidad, conocida como redshift drift y potencialmente medible con la tecnología actual, nos ofrecería la posibilidad de distinguir modelos cosmológicos [139]. Es importante entonces estudiar las predicciones teóricas para este observable en diferentes escenarios cosmológicos, con el fin de poder cotejarlas en un futuro próximo con datos observacionales.

En esta dirección, estudiaremos en el capítulo 3 el redshift drift a partir de dos aproximaciones para fuentes cercanas. La primera está basada en el formalismo covariante $1+3$, que posee la ventaja de ser independiente de las coordenadas utilizadas y del punto de observación. La segunda, por su parte, consiste en estudiar el redshift drift a partir de consideraciones cosmográficas, esto es, que no involucren la dinámica subyacente en un dado modelo.

\section{El desarrollo de la inflación en presencia de regiones no homogéneas.}

Una de las hipótesis más importantes en la que están basados los modelos de inflación es la de suponer que cualquier irregularidad inicial es homogeneizada debido a la violenta expansión que caracteriza a la etapa inflacionaria [63]. Si bien existen argumentos teóricos que justifican esta hipótesis, no hay una prueba definitiva de que la inflación tenga esta propiedad para cualquier tipo de escenario inicial.

Con esta motivación, nos proponemos estudiar si una región de vacío del espacio-tiempo puede desarrolar una etapa de inflación en ambientes no homogéneos, con el objetivo de comprobar si alguna distribución primordial no homogénea de materia o radiación puede inhibir el proceso de inflación. Los fondos no homogéneos serán descriptos por las soluciones de LTB y de Lemaître, para caracterizar, respectivamente, la evolución en ambientes con polvo y radiación. Este trabajo será presentado en el capítulo 4.

3. Modelos con distribución inicial no homogénea de materia y radiación.

En el marco de los modelos de void, escenarios cosmológicos más complejos han sido sugeridos para modelar los efectos de la radiación sobre la evolución temprana de la geometría del universo [133]. Este problema conduce a la necesidad de considerar modelos con dos fluidos no comóviles, esto es, materia y radiación. Un análisis cualitativo de este tipo de modelos fue presentado en [133], y un primer tratamiento de este problema (sin incluir los momentos multipolares de la distribución de la radiación) fue realizado en la referencia [140]. 
Siguiendo los trabajos mencionados anteriormente, estudiaremos la evolución de un modelo de dos fluidos con simetría esférica (en donde la radiación y la materia son no comóviles), que incluya los efectos de la presión anisótropa de la radiación sobre la evolución de la geometría. Este trabajo será presentado en el capítulo 5.

Para concluir, discutiremos en el capítulo 6 las prespectivas futuras sobre la investigación de modelos cosmológicos no homogéneos, así como también las líneas de trabajo que se desprenden como continuación natural del estudio realizado en esta tesis. 
"Por último, cansado de esperar, se lamentó ante Melquíades del fracaso de su iniciativa, y el gitano dio entonces una prueba convincente de honradez: le devolvió los doblones a cambio de la lupa, y le dejó además unos mapas portugueses y varios instrumentos de navegación.

De su puño y letra escribió una apretada síntesis de los estudios del monje Hermann, que dejó a su disposición para que pudiera servirse del astrolabio, la brújula y el sextante. José Arcadio Buendía pasó los largos meses de lluvia encerrado en un cuartito que construyó en el fondo de la casa para que nadie perturbara sus experimentos. Habiendo abandonado por completo las obligaciones domésticas, permaneció noches enteras en el patio vigilando el curso de los astros, y estuvo a punto de contraer una insolación por tratar de establecer un método exacto para encontrar el mediodía. Cuando se hizo experto en el uso y manejo de sus instrumentos, tuvo una noción del espacio que le permitió navegar por mares incógnitos, visitar territorios deshabitados y trabar relación con seres espléndidos, sin necesidad de abandonar su gabinete. Fue esa la época en que adquirió el hábito de hablar a solas, paseándose por la casa sin hacer caso de nadie, mientras Úrsula y los niños se partían el espinazo en la huerta cuidando el plátano y la malanga, la yuca y el ñame, la ahuyama y la berenjena. De pronto, sin ningún anuncio, su actividad febril se interrumpió y fue sustituida por una especie de fascinación. Estuvo varios días como hechizado, repitiéndose a si mismo en voz baja un sartal de asombrosas conjeturas, sin dar crédito a su propio entendimiento. Por fin, un martes de diciembre, a la hora del almuerzo, soltó de un golpe toda la carga de su tormento. Los niños habían de recordar por el resto de su vida la augusta solemnidad con que su padre se sentó a la cabecera de la mesa, temblando de fiebre, devastado por la prolongada vigilia y por el encono de su imaginación, y les reveló su descubrimiento: -La tierra es redonda como una naranja. (...)"

Cien años de soledad (1967)

Gabriel García Márquez 


\section{Capítulo 2}

\section{Soluciones no homogéneas de las ecuaciones de Einstein}

Presentaremos en este capítulo una breve introducción a las soluciones no homogéneas de las ecuaciones de Einstein con simetría esférica, así como a los modelos cosmológicos construidos a partir de las mismas.

La primer solución que analizaremos es la de Lemaître-Tolman-Bondi (LTB), cuya fuente es un fluido perfecto con presión nula. La expresión analítica de esta solución puede ser obtenida en forma exacta, lo que permite imponer restricciones sobre ciertas funciones que la caracterizan, con el fin de evitar singularidades y comportamientos no deseados en escenarios cosmológicos o astrofísicos. Analizaremos, además, las propiedades generales de la propagación de la luz en esta geometría a partir del estudio de las geodésicas nulas. Por último, presentaremos algunas aplicaciones de la solución de LTB a problemas cosmológicos y astrofísicos.

La geometría con simetría esférica descripta por la solución de Lemaître admite como fuente un fluido con distribuciones espaciales de densidad y presión no homogéneas. Esta solución, poco explorada hasta el momento, permite el estudio de modelos con materia ultrarelativista. Analizaremos aquí algunas de sus propiedades generales.

A modo de complemento de las soluciones con simetría esférica, presentaremos también la solución de Székeres (sin simetrías definidas) y algunas de sus propiedades más generales. 


\subsection{La solución de Lemaître-Tolman-Bondi}

La solución no homogénea de las ecuaciones de Einstein con simetría esférica y polvo como fuente (es decir, un fluido perfecto con presión nula) fue encontrada y estudiada en el año 1933 por A. G. Lemaître [106], y más tarde por R. C. Tolman [107] y H. Bondi [98], razón por la cual es conocida como solución de Lemaître-Tolman-Bondi (en adelante, LTB). La misma está caracterizada en coordenadas comóviles sincronizadas ${ }^{1}$ por el elemento de línea

$$
\mathrm{d} s^{2}=c^{2} \mathrm{~d} t^{2}-\frac{\left[R^{\prime}(t, r)\right]^{2}}{1+2 E(r)} \mathrm{d} r^{2}-[R(t, r)]^{2}\left(\mathrm{~d} \theta^{2}+\operatorname{sen}^{2} \theta \mathrm{d} \varphi^{2}\right)
$$

en donde el símbolo ' indica derivadas respecto a la coordenada radial $r$, y $E(r)$ es una función arbitraria que, como mostraremos más adelante, determina la curvatura de las hipersuperficies con $t=$ cte. Notemos que, para que la métrica (2.1) mantenga los signos adecuados, es necesario que $E(r)>-1 / 2$ para todo $r .^{2}$ La densidad $\rho(t, r)$, que caracteriza al fluido perfecto con presión nula, resulta

$$
\kappa \rho(t, r)=\frac{2 M^{\prime}(r)}{R^{\prime}(t, r)[R(t, r)]^{2}},
$$

con $\kappa=8 \pi G / c^{4}$. En adelante, trabajaremos con la elección de unidades acorde al Sistema Natural de Unidades, esto es, $c=1, G=1 \mathrm{y} \hbar=1$. La función métrica $R(t, r)$ queda determinada por la siguiente ecuación [16]

$$
[\dot{R}(t, r)]^{2}=2 E(r)+\frac{2 M(r)}{R(t, r)}+\frac{\Lambda}{3}[R(t, r)]^{2},
$$

en donde el símbolo 'indica derivadas respecto a la coordenada temporal $t$, y $M(r)$ es otra función arbitraria de integración, que representa la masa gravitacional activa que genera el campo gravitacional. Si bien la solución formal admite una constante cosmológica no nula [141], en el contexto de esta tesis trabajaremos con $\Lambda=0$, a menos que explicitemos lo contrario. $^{3}$

Las ecuaciones (2.3) y (2.2) son obtenidas trabajando algebraicamente con las ecuaciones de Einstein, e imponiendo la condición de simetría esférica y las restricciones $\dot{R} \neq 0$ y $R^{\prime} \neq 0$. El caso $\dot{R}=0$ no tiene interés para el estudio de soluciones cosmológicas no homogéneas, pues requiere que o bien $R^{\prime}=0$ (solución de Nariai $(1950)[142,143]$ ) o bien que la densidad sea constante [16]. Sin embargo, el caso $R^{\prime}=0$ da lugar a soluciones interesantes que estudiaremos en detalle en la sección 2.1.1.

La ecuación (2.3) admite una familia de soluciones exactas para $R(t, r)$, cuya evolución

\footnotetext{
${ }^{1}$ Las coordenadas comóviles sincronizadas son aquellas coordenadas comóviles en las cuales las componentes cruzadas espacio-temporales de la métrica resultan nulas. Esta elección de coordenadas es viable siempre que el campo de velocidades comóviles $u^{\alpha}$ tenga rotación nula. El sistema de referencia es denominado entonces comóvil sincronizado, y puede probarse que la rotación nula es una condición necesaria y suficiente para que dichas coordenadas existan en espacio-tiempos con simetría esférica y un fluido perfecto como fuente [16].

${ }^{2} \mathrm{El}$ caso con $E(r)=-1 / 2$ está asociado a la existencia de los llamados agujeros de gusano [16].

${ }^{3}$ Notemos que la ecuación (2.3) con $\Lambda=0$ es formalmente idéntica a la ecuación newtoniana del movimiento radial en un potencial coulombiano. En esta analogía coulombiana, $M(r)$ juega el papel de la masa gravitacional activa dentro de una cáscara de radio constante, y $E(r)$ representa la energía total dentro de esta la misma [16].
} 
depende del signo de la función $E(r)$. La expresión analítica de dichas soluciones resulta ${ }^{4}$

$$
\begin{aligned}
& \left\{\begin{aligned}
R(t, r) & =-\frac{M(r)}{2 E(r)}(1-\cos \eta) \\
\eta-\operatorname{sen} \eta & =\frac{[-2 E(r)]^{3 / 2}}{M(r)}\left(t-t_{B}(r)\right)
\end{aligned} \quad \text {, si } E(r)<0 ;\right. \\
& R(t, r)=\left[\frac{9}{2} M(r)\left(t-t_{B}(r)\right)^{2}\right]^{1 / 3}, \text { si } E(r)=0 \\
& \left\{\begin{aligned}
R(t, r) & =-\frac{M(r)}{2 E(r)}(\cosh \eta-1) \\
\operatorname{senh} \eta-\eta & =\frac{[2 E(r)]^{3 / 2}}{M(r)}\left(t-t_{B}(r)\right)
\end{aligned} \quad \text {, si } E(r)>0 .\right.
\end{aligned}
$$

Las soluciones anteriores dependen de una función de integración adicional, $t_{B}(r)$, que aparece combinada con la coordenada temporal en la forma $\left(t-t_{B}(r)\right)$. Esta función, conocida en la literatura como función de bang-time, aparece al integrar la ecuación (2.3), esto es,

$$
\int_{0}^{R} \frac{\mathrm{d} \tilde{R}}{\sqrt{2 E+2 M / \tilde{R}+\frac{1}{3} \Lambda \tilde{R}^{2}}}=t-t_{B}(r) .
$$

Podemos interpretar la integral anterior como el valor de la coordenada $t$ para el cual la expansión diverge, y resulta entonces dependiente de la posición $r$ (notemos la diferencia con la solución homogénea de FLRW, en la cual la coordenada correspondiente al llamado bangtime es constante para todo $r$ ). En principio no existen a priori restricciones que determinen el signo de $t_{B}(r)$, por lo que su elección queda supeditada al contexto particular de cada problema.

Las soluciones paramétricas (2.4) y (2.6) pueden también reescribirse, respectivamente, como [109]

$$
\left\{\begin{array}{c}
t=t_{B}+\frac{M}{(-2 E)^{(3 / 2)}}\left[\arccos \left(1+2 \frac{E R}{M}\right)-2 \sqrt{\frac{-E R}{M}\left(1+\frac{E R}{M}\right)}\right], \\
0 \leq \eta \leq \pi \\
t=t_{B}+\frac{M}{(-2 E)^{(3 / 2)}}\left[\pi+\arccos \left(-1-2 \frac{E R}{M}\right)+2 \sqrt{\frac{-E R}{M}\left(1+\frac{E R}{M}\right)}\right], \\
\pi \leq \eta \leq 2 \pi
\end{array}\right.
$$

$\mathrm{y}$

$$
t=t_{B}+\frac{M}{(2 E)^{(3 / 2)}}\left[2 \sqrt{\frac{E R}{M}\left(1+\frac{E R}{M}\right)}-\operatorname{arccosh}\left(1+2 \frac{E R}{M}\right)\right] .
$$

(expansión)

La familia de soluciones de LTB depende entonces de tres funciones arbitrarias de integración, a saber, $E(r), M(r)$ y $t_{B}(r)$. Sin embargo, las ecuaciones (2.4)-(2.6) son invariantes ante

\footnotetext{
${ }^{4}$ Estrictamente, la clasificación de soluciones está asociada al signo de la cantidad $E / M^{2 / 3}$, dado que las condiciones de regularidad en el centro de simetría imponen $E=0$ siempre que $M=0$ [16].
} 
transformaciones de coordenadas de la forma $\tilde{r}=g(r)$, con lo cual una de las tres funciones puede ser fijada a partir de esta invarianza, según convenga en el problema con el que estemos trabajando. Las soluciones (2.4)-(2.6) presentan, además, la misma estructura algebraica que las soluciones de las ecuaciones de Friedmann-Lemaître. La solución de FLRW puede ser recuperada en el límite homogéneo $\rho^{\prime}=0$, con

$$
\begin{aligned}
t_{B}(r) & =\text { cte } \\
\left(|E(r)|^{3 / 2} / M(r)\right) & =\text { cte }
\end{aligned}
$$

y la siguiente elección de coordenadas

$$
M=M_{0} r^{3}\left(\Rightarrow E=E_{0} r^{2}\right),
$$

con $M_{0}$ y $E_{0}$ constantes. $^{5}$

Como mencionamos anteriormente, la función $E(r)$ presenta una relación directa con la curvatura de los subespacios con $t=$ cte. Sobre estas hipersuperficies, la función $R$ depende sólo de $r$ y puede ser uttilizada entonces como coordenada radial (nuevamente, haciendo uso de la invariancia ante una transformación de coordenadas del tipo $\tilde{r}=g(r)$ ). Considerando las tétradas ortogonales a los subespacios con $t=$ cte, dadas por $\mathrm{e}^{1}=\mathrm{d} R / \sqrt{1+2 E}, \mathrm{e}^{2}=R \mathrm{~d} \theta$, y $\mathrm{e}^{3}=R \operatorname{sen} \theta \mathrm{d} \varphi$, las únicas componentes no nulas del tensor de Riemann tridimensional resultan

$$
R_{1212}=-\frac{\mathrm{d} E / \mathrm{d} R}{R}=R_{1313}, \quad \mathrm{y} \quad R_{2323}=-\frac{2 E}{R^{2}} .
$$

Vemos entonces que todo subespacio con $t=$ cte y $E=0$ es plano. Por otra parte, si $E / R^{2}=$ cte, la curvatura también es constante y su signo es igual al signo de $-E$. Por lo tanto, encontramos que $(-E)$ es justamente una medida de la curvatura de los subespacios con $t=$ cte. A diferencia de las soluciones de Friedmann-Lemaître, la curvatura en este caso es local. Es decir, depende de $r$ y, en particular, puede resultar positiva en alguna región del espacio y negativa en otra. Esto nos muestra que la distinción que usualmente hacemos entre la familia de modelos de FLRW (en relación a los valores de la constante de curvatura $k$ ) es una peculiaridad de la solución homogénea, y no una propiedad del universo físico que estamos intentando describir. Las soluciones (2.4)-(2.6) pueden utilizarse simultáneamente para estudiar distintas regiones del mismo espacio-tiempo, cada una con su curvatura característica.

Por último, teniendo en cuenta el formalismo covariante presentado en el apéndice A y las definiciones allí detalladas, caracterizamos a la velocidad del fluido con el 4-vector $u^{\alpha}=\delta_{0}^{\alpha}$.

\footnotetext{
${ }^{5}$ Notemos que, si bien este límite depende de las coordenadas elegidas, la condición $\rho^{\prime}=0$ es la que asegura la hipótesis de homogeneidad y resulta invariante ante transformaciones de la coordenada $r$. Para analizar esto, consideremos $M(r)=\tilde{r}$ (esto es, que para cada intervalo de $r$ en que $M^{\prime}$ no cambie de signo, $M$ puede ser utilizada como coordenada radial). Luego, reescribimos la ecuación (2.2) como

$$
\kappa \rho=\frac{6}{\mathrm{~d}\left(R^{3}\right) / \mathrm{d} M} \Leftrightarrow R^{3}-R^{3}\left(M_{0}\right)=\int_{M_{0}}^{M} \frac{6}{\kappa \rho(\tilde{M})} d \tilde{M} .
$$

La condición de homogeneidad espacial es $\mathrm{d} \rho / \mathrm{d} M=0$, es decir, $\mathrm{d}^{2}\left(R^{3}\right) / \mathrm{d} M^{2}=0$. Trabajando con estas restricciones, encontramos que son equivalentes a pedir $\frac{E}{M^{2 / 3}}=$ cte y $t_{B}=$ cte, definiendo en forma invariante el límite homogéneo [16].
} 
Luego, las cantidades cinemáticas de la solución de LTB resultan

$$
\begin{aligned}
\Theta & =\frac{\dot{R}^{\prime} R+2 \dot{R} R^{\prime}}{R R^{\prime}}, \\
\sigma_{\beta}^{\alpha} & =\frac{1}{3}\left(\frac{\dot{R}^{\prime} R-2 \dot{R} R^{\prime}}{R R^{\prime}}\right) \operatorname{diag}(0,2,-1,-1), \\
0 & =a^{\alpha}=\omega_{\beta}^{\alpha} .
\end{aligned}
$$

Asimismo, las partes eléctrica y magnética del tensor de Weyl son, respectivamente,

$$
\begin{aligned}
E_{\beta}^{\alpha} & =\frac{1}{6}\left(\frac{R\left[-\ddot{R}^{\prime} R+\dot{R} \dot{R}^{\prime}-E^{\prime}\right]+R^{\prime}\left[\ddot{R} R-\dot{R}^{2}+2 E\right]}{R R^{\prime}}\right) \operatorname{diag}(0,2,-1,-1), \mathrm{y}(2.1 \\
H_{\beta}^{\alpha} & =0
\end{aligned}
$$

\subsubsection{Condiciones de regularidad}

Dado que la métrica no homogénea de LTB involucra funciones no triviales de las coordenadas temporal y radial, es importante estudiar bajo qué condiciones estas funciones describen soluciones regulares. ${ }^{6}$

\section{Regularidad en el centro de simetría}

En el centro de simetría (identificado con el valor de coordenada radial $r=r_{o}$ ) tenemos que $M\left(r_{o}\right)=R\left(t, r_{o}\right)=0$ para todo tiempo $t$. La condición de regularidad en estos puntos puede ser estudiada fácilmente también a partir de las componentes del tensor de Riemann en tétradas [109]. Del análisis de los escalares (2.23)-(2.25), notamos que la condición para evitar divergencias en la curvatura de aquellos puntos con $M\left(r_{o}\right)=R\left(t, r_{o}\right)=0$ es simplemente

$$
\lim _{r \rightarrow r_{o}} \frac{M}{R^{3}}<0 .
$$

Luego, utilizando la ecuación (2.2), tenemos que

$$
\lim _{r \rightarrow r_{o}} \frac{M}{R^{3}}=\lim _{r \rightarrow r_{o}} \frac{M^{\prime}}{3 R^{2} R^{\prime}}=\frac{1}{6} \kappa \rho\left(t, r_{o}\right)<0 .
$$

Es decir, que la curvatura será finita en el origen siempre que $\rho\left(t, r_{o}\right)$ también lo sea. Asimismo, las condiciones de regularidad que obtenemos para el comportamiento de las funciones en torno al centro de simetría son [109]

$$
\begin{aligned}
R & =\beta(t) M^{1 / 3}+\mathcal{O}_{1 / 3}(M) \quad(\text { en superficies con } t=\text { cte }) \\
E & =\gamma M^{2 / 3}+\mathcal{O}_{2 / 3}(M) \\
t_{B} & =\tau_{0}+\tau+\mathcal{O}_{1 / 3}(M), \\
\kappa \rho & =\frac{6}{\beta^{3}}+\mathcal{O}_{1 / 3}(M) \quad(\text { en superficies con } t=\text { cte })
\end{aligned}
$$

en donde $\mathcal{O}_{d}(M)$ indica alguna función con la propiedad $\operatorname{lím}_{M \rightarrow 0}\left(\mathcal{O}_{d} / M^{d}\right)=0, \beta(t)$ es una función arbitraria no nula, y $\gamma, \tau_{0}$ y $\tau$ son constantes arbitrarias, con $\tau>0$.

\footnotetext{
${ }^{6}$ Un análisis de esta solución a partir de sistemas dinámicos fue realizado en [144].
} 


\section{Otras condiciones de regularidad}

La ecuación (2.2), que define la densidad del fluido en términos de las funciones de la métrica, diverge cuando $R=0 \neq M^{\prime}$ o $R^{\prime}=0 \neq M^{\prime}$. El primer caso corresponde a una singularidad análoga al punto de big-bang para la solución de LTB (esto es, que el factor de escala de la parte angular de la métrica resulta nulo), y tendrá lugar siempre que trabajemos con $\Lambda=0$. La segunda condición está asociada a lo que usualmente denominamos como shell crossing: escenarios en los que la densidad de masa crece hasta alcanzar un límite infinito (en donde eventualmente cambia su signo y pasa a tener un valor negativo, si $R^{\prime}$ también cambia de signo). En estos puntos, la distancia radial geodésica $\left(\sqrt{\left|g_{r r}\right| \mathrm{d} r}\right)$ entre dos puntos $\left(t_{0}, r_{0}, \theta_{0}, \varphi_{0}\right)$ y $\left(t_{0}, r_{0}+\mathrm{d} r, \theta_{0}, \varphi_{0}\right)$ tiende a cero y, en consecuencia, hipersuperficies con $r=$ cte con distintos valores de la coordenada radial coinciden [16].

Para profundizar el análisis de las regiones con shell crossing, estudiaremos las componentes escalares del tensor de Riemann expresadas en las coordenadas tétradas de la métrica $\left(e^{0}=\mathrm{d} t, e^{1}=\left(R^{\prime} /(\sqrt{1+2 E}) \mathrm{d} r, e^{2}=R \mathrm{~d} \theta\right.\right.$ y $\left.e^{3}=R \operatorname{sen} \theta \mathrm{d} \varphi\right)$, dadas por [16, 109]

$$
\begin{aligned}
& R_{0101}=\frac{2 M}{R^{3}}-\frac{M^{\prime}}{R^{2} R^{\prime}}, \\
& R_{0202}=R_{0303}=\frac{1}{2} R_{2323}=-\frac{M}{R^{3}}-\frac{M^{\prime}}{R^{2} R^{\prime}}, \\
& R_{1212}=R_{1313}=\frac{M}{R^{3}}-\frac{M^{\prime}}{R^{2} R^{\prime}} .
\end{aligned}
$$

Las regiones con puntos singulares en la curvatura pueden ser evitadas con una elección apropiada de las funciones arbitrarias $E(r), M(r)$ y $t_{B}(r)$. Para esto, podemos adoptar dos criterios:

1. fijar las funciones de forma tal que resulte $R^{\prime} \neq 0$ en todas las regiones en donde nos interesa aplicar la solución de LTB; o

2. elegir las funciones arbitrarias de forma tal que $R^{\prime}=0$ sólo en aquellos puntos $r=r_{s}$ en donde $M^{\prime}\left(r_{s}\right)=0$ y el $\lim _{r \rightarrow r_{s}}\left|M^{\prime} / R^{\prime}\right|<\infty$.

En el primer caso, la condición $R^{\prime} \neq 0$ impone ciertas restricciones sobre las funciones $E(r)$, $M(r)$ y $t_{B}(r)$ [122]. Para cada una de las soluciones (2.4)-(2.6), las condiciones necesarias y suficientes que deben cumplir las funciones del modelo para evitar regiones con shell crossing si $R^{\prime}>0$ son las siguientes [16]

$$
\begin{aligned}
& \begin{cases}M^{\prime}>0 & \\
\left(t_{B}\right)^{\prime}<0 & \text {, si } \mathrm{E}<0 ; \\
2 \pi\left(\frac{3}{2} \frac{E_{, r}}{E}-\frac{M^{\prime}}{M}\right)-\frac{(-2 E)^{3 / 2}}{M} t_{B . r}<0 & \end{cases} \\
& \begin{cases}M^{\prime}>0 & \text {, si } \mathrm{E}=0 ; \mathrm{y}\end{cases} \\
& \begin{cases}t_{B, r}<0 & \text {, si } \mathrm{E}>0 . \\
M^{\prime}>0 & \end{cases}
\end{aligned}
$$


Las regiones en las que $R^{\prime}<0$ imponen restricciones análogas, pero con las desigualdades invertidas.

Para estudiar el segundo caso, en el que $R^{\prime}=0$ sólo en puntos $r=r_{s}$ en donde $M^{\prime}=0$ y el $\operatorname{lím}_{r \rightarrow r_{s}} M^{\prime} / R^{\prime}<\infty$, suponemos que $t \geqslant t_{B}$ [122]. La condición necesaria para que densidad de masa permanezca finita en $r=r_{s}$ es

$$
M^{\prime}\left(r_{s}\right)=0
$$

Por otra parte, dado que la función $M$ no tiene dependencia con la coordenada temporal, la densidad de masa podrá ser finita siempre que $R^{\prime}=0$ tampoco dependa de $t$. Además, debe satisfacerse el siguiente límite

$$
\lim _{r \rightarrow r_{s}} M^{\prime} / R^{\prime}<\infty
$$

Este límite resulta simple de trabajar si elegimos $\tilde{r}=M(r)$ como coordenada radial (haciendo uso de la invariancia ante transformaciones de coordenadas radiales que posee la métrica). De esta forma, la divergencia del tipo $\left(M^{\prime} / R^{2} R^{\prime}\right)$ puede ser evitada si [16]

$$
\begin{gathered}
\lim _{r \rightarrow r_{s}} \frac{M^{\prime}(r)}{R^{2}(t, r) R^{\prime}(t, r)}=\lim _{M(r) \rightarrow M\left(r_{s}\right)} \frac{1}{R^{2}(t, M) \mathrm{d} R(t, M) / \mathrm{d} M}=\lim _{M(r) \rightarrow M\left(r_{s}\right)} \frac{1}{\mathrm{~d}\left[R^{3}(t, M)\right] / \mathrm{d} M}<\infty \\
\Rightarrow \lim _{M(r) \rightarrow M\left(r_{s}\right)} \mathrm{d}\left[R^{3}(t, M)\right] / \mathrm{d} M>0
\end{gathered}
$$

Para el caso con $E=0$, la condición (2.31) puede escribirse como

$$
R^{\prime}=\frac{1}{3} R^{-2 / 3}\left[M^{\prime}\left(t-t_{B}\right)^{2}-2 M t_{B, r}\right]
$$

Es decir, que basta con imponer $t_{B, r}\left(r_{s}\right)=0$. Para los casos $E<0$ y $E>0$, la derivada de la solución en forma paramétrica resulta

$$
R^{\prime}=\left(\frac{M^{\prime}}{M}-\frac{E^{\prime}}{E}\right) R+\left[\left(\frac{3}{2} \frac{E^{\prime}}{E}-\frac{M^{\prime}}{M}\right)\left(t-t_{B}\right)-t_{B, r}\right] \dot{R}
$$

Luego, teniendo en cuenta que $\dot{R}$ y $R$ son funciones de $r$ y $t$ no idénticas, la condición $R^{\prime}\left(r_{s}\right)=0$ es satisfecha siempre que cada uno de los términos de la derecha sean nulos, esto es, que $E^{\prime}\left(r_{s}\right)=0$ y $t_{B, r}\left(r_{s}\right)=0$.

Estas son las condiciones necesarias para evitar shell-crossing en las soluciones de LTB para aquellos puntos en los que $R^{\prime}=0$. Las restricciones sobre $E(r), M(r)$ y $t_{B}(r)$ para encontrar condiciones suficientes que eviten el comportamiento de shell crossing son menos generales y deben ser estudiadas para cada caso particular a partir de la condición general dada por la ecuación (2.31) [16]. ${ }^{7}$

\footnotetext{
${ }^{7}$ Notemos que si en el caso con $R^{\prime}=0$ las condiciones necesarias para evitar shell crossing son satisfechas y, además, $1+2 E\left(r_{s}\right) \neq 0$, la componente $g_{r r}$ de la métrica resulta nula. Sin embargo, supusimos que el cociente $M^{\prime} / R^{\prime}$ es finito en $r=r_{s}$, es decir, que la curvatura no es singular en este punto. Esto implica que $r=r_{s}$ es un punto singular en el sistema de coordenadas utilizado y, en consecuencia, puede ser removido haciendo una transformación de coordenadas adecuada (que dará lugar a $R_{r}\left(r_{s}\right) \neq 0$ ) [16].
} 


\subsubsection{Propagación de la luz en la geometría de LTB}

Con el fin de comparar los modelos cosmológicos con diferentes observaciones astronómicas, es imprescindible el estudio de las ecuaciones diferenciales que determinan las geodésicas nulas en la métrica de LTB, así como también el análisis del corrimiento al rojo en la frecuencia de los fotones (en adelante, redshift) que sufre la luz proveniente de una dada fuente. Abordaremos en esta sección el estudio de la propagación de la luz para el caso de geodésicas radiales. El caso más general, en el que las geodésicas inciden fuera del centro de simetría, está presentado en el apéndice B. Este último caso resulta de interés para estudiar cantidades observables que pueden ser no nulas si el modelo se aparta de la simetría esférica.

Teniendo en cuenta la libertad que ofrece la solución de LTB para fijar alguna de sus funciones arbitrarias, trabajaremos considerando $t>t_{B}(r) .{ }^{8}$ Partiendo de la métrica (2.1), la geodésica nula que describe la trayectoria de un rayo de luz emitido por una fuente con coordenadas $(t, r, \theta, \phi)$, y que viaja en forma radial hasta el centro de simetría del sistema, satisface la ecuación

$$
\frac{\mathrm{d} t}{\mathrm{~d} r}=-\frac{R^{\prime}(t, r)}{\sqrt{1+2 E(r)}} .
$$

Para el cálculo del redshift, consideremos ahora dos fotones de luz provenientes de la misma fuente medidos en los instantes $t_{1}=T(r)$ y $t_{2}=T(r)+\tau(r)$, respectivamente, en donde $\tau(r)$ es un intervalo de tiempo pequeño $(\tau(r) / T(r) \ll 1)$. Notemos que la dirección de incidencia de ambos fotones coincide por estar trabajando con geodésicas radiales. La expresión (2.34) resulta, para cada fotón,

$$
\begin{aligned}
\frac{\mathrm{d} T}{\mathrm{~d} r} & =-\frac{R^{\prime}(T(r), r)}{\sqrt{1+2 E(r)}}, \mathrm{y} \\
\frac{\mathrm{d}(T+\tau)}{\mathrm{d} r} & =-\frac{R^{\prime}(T(r)+\tau(r), r)}{\sqrt{1+2 E(r)}} .
\end{aligned}
$$

Trabajando sólo a primer orden en $\tau$, podemos reescribir

$$
R^{\prime}(T(r)+\tau(r), r) \simeq R^{\prime}(T(r), r)+\tau(r) R^{\prime \prime}(T(r), r) .
$$

Usando esta aproximación y la ecuación (2.35), la expresión (2.36) resulta [16]

$$
\frac{\mathrm{d} \tau}{\mathrm{d} r}=-\tau(r) \frac{\dot{R}^{\prime}(T(r), r)}{\sqrt{1+2 E(r)}} .
$$

Por otra parte, si $\tau(r)$ es el período de la onda emitido por una fuente con coordenada radial $r, \mathrm{y} \tau\left(r_{o}\right)$ es el período medido en el centro de simetría, definimos el redshift $z(r)$ de la fuente como

$$
z(r) \equiv \frac{\tau\left(r_{o}\right)}{\tau(r)}-1
$$

Derivando esta expresión respecto de la coordenada $r$, y teniendo en cuenta la ecuación (2.38), resulta [16]

$$
\frac{1}{1+z(r)} \frac{\mathrm{d} z}{\mathrm{~d} r}=\frac{\dot{R}^{\prime}(T(r), r)}{\sqrt{1+2 E(r)}},
$$

\footnotetext{
${ }^{8}$ Podemos elegir siempre una traslación adecuada en las hipersuperficies $t=$ cte de forma tal que esta condición sea satisfecha. En este contexto, tomar valores crecientes de $t$ implica ir desde el pasado hacia el futuro [109].
} 
o bien

$$
\frac{\mathrm{d} \ln (z)}{\mathrm{d} r}=\int_{r}^{r_{o}} \frac{\dot{R}^{\prime}(T(r), r)}{\sqrt{1+2 E(r)}} \mathrm{d} r .
$$

Esta fórmula expresa el redshift de la fuente parametrizado con la coordenada radial $r$. La integración sobre la trayectoria geodésica de los fotones será, en general, calculada en forma numérica debido a la complejidad de las funciones $R(t, r)$ que caracterizan a cada familia de soluciones de LTB.

Notemos que la ecuación (2.40) puede ser utilizada para parametrizar la geodésica nula a través de $z$, en lugar de utilizar la coordenada radial. Dicha opción puede ser considerada siempre que $z(r)$ resulte, para el modelo con el que trabajemos, una función biyectiva, al menos en el mínimo volumen esférico centrado en el observador que encierre a la fuente. Trabajando entonces con este cambio de variables en la ecuación (2.34), obtenemos [109]

$$
\frac{\mathrm{d} t}{\mathrm{~d} z}=-\frac{R^{\prime}(t(r), r)}{(1+z) \dot{R}^{\prime}(t(r), r)}
$$

De esta forma, las geodésicas nulas serán la solución del sistema de ecuaciones diferenciales acopladas $(2.40)$ y $(2.42)$, cuya integración debe ser realizada desde $z_{1}=z_{\text {fuente }}$ hasta $z_{2}=$ $z_{o}=0$.

\subsubsection{Aplicaciones de la solución de LTB}

Las soluciones de LTB han sido estudiadas para abordar diferentes problemas de cosmología y astrofísica $[16,109]$. En relación a los escenarios cosmológicos, la posibilidad de construir modelos no homogéneos, basados en esta solución y que puedan describir la curva de luminosidad de las SN tipo Ia sin la necesidad de incorporar energía oscura, ha sido explorada con creciente interés [101, 126]. En esta dirección, podemos destacar a los llamados modelos de void, caracterizados por una distribución no homogénea con una subdensidad en el centro de simetría de escalas de Gpcs en etapas tardías de la evolución del universo. Estos modelos serán estudiados en detalle en el capítulo 5 de esta tesis.

Por otra parte, soluciones con perfiles más generales pueden ser utilizadas para estudiar diversos escenarios con simetría esférica. Este es el caso del trabajo que presentaremos en el capítulo 4 de esta tesis, en donde perfiles de materia más complejos son utilizados para modelar condiciones iniciales no homogéneas en las que podría haber tenido lugar la etapa de inflación.

En un contexto astrofísico, la solución de LTB ha sido utilizada también para estudiar la representación de agujeros negros dinámicos, con un comportamiento asintótico tipo FLRW [145, 146, 147], y modelos de colapso gravitacional para el caso de fluidos de polvo, no homogéneos, con simetría esférica y sin rotación [148, 149].

\subsection{La solución de Lemaître}

A diferencia de las soluciones presentadas anteriormente, la geometría de Lemaître [106] ha sido hasta el momento poco estudiada en la literatura. Sin embargo, representa la descripción más completa de soluciones con simetría esférica y un fluido perfecto como fuente, con distribuciones de densidad y presión no homogéneas. 
El elemento de línea que describe la métrica en coordenadas comóviles viene dado por

$$
\mathrm{d} s^{2}=\mathrm{e}^{A(t, r)} \mathrm{d} t^{2}-\mathrm{e}^{B(t, r)} \mathrm{d} r^{2}-R^{2}(t, r) \mathrm{d} \Omega^{2} .
$$

Las ecuaciones de Einstein resultan

$$
\begin{aligned}
R^{2}(t, r) R^{\prime}(t, r) \rho(t, r) & =2 M^{\prime}(t, r), \\
R^{2}(t, r) \dot{R}(t, r) p(t, r) & =-2 \dot{M}(t, r),
\end{aligned}
$$

en donde los símbolos ${ }^{\prime} \mathrm{y}^{\prime}$ indican, como antes, derivadas respecto de las coordenadas $t$ y $r$, respectivamente. Las funciones $\rho(t, r)$ y $p(t, r)$ representan, respectivamente, la densidad y la presión del fluido. La función de integración $M(t, r)$ queda definida por la ecuación

$$
2 M(t, r)=R(t, r)+\mathrm{e}^{-A(t, r)} \dot{R}^{2}(t, r) R(t, r)-\mathrm{e}^{-B(t, r)} R^{\prime 2}(t, r) R(t, r)-\frac{\Lambda R^{3}(t, r)}{3} .
$$

A partir de la conservación de $T_{\mu \nu}$, tenemos

$$
\begin{aligned}
A^{\prime}(t, r) & =-\frac{2 p^{\prime}(t, r)}{\rho(t, r)+p(t, r)}, \\
\mathrm{e}^{B(t, r)} & =\frac{R^{\prime 2}(t, r)}{1+2 E(r)} \exp \left(\int_{t_{0}}^{t} \frac{2 \dot{R}(\tilde{t}, r)}{[\epsilon(\tilde{t}, r)+p(\tilde{t}, r)] R^{\prime}(\tilde{t}, r)} p^{\prime}(\tilde{t}, r) \mathrm{d} \tilde{t}\right),
\end{aligned}
$$

siendo $E(r)$ una función arbitraria asociada a la curvatura local [150]. Notemos que las expresiones anteriores pueden ser reducidas al caso de LTB cuando $p(t, r)=p^{\prime}(t, r)=0$, esto es, cuando $\mathrm{e}^{A(t, r)}=1$ y e $\mathrm{e}^{B(t, r)}=R^{\prime 2}(t, r) /[1+2 E(r)]$. La métrica de FLRW es recuperada en el límite $R(t, r) \rightarrow a(t) r, M(t, r) \rightarrow M_{0} r^{3}$ y $E(r) \rightarrow E_{0} r^{2}$.

Una característica particular de esta geometría es que las regiones no homogéneas no quedan confinadas a la coordenada radial inicial, como en el caso de la solución de LTB. Para ver esto, consideremos la ecuación de evolución (2.46), que podemos reescribir de la forma siguiente

$$
\mathrm{e}^{-A} \dot{R}^{2}=\frac{2 M}{R}+\frac{1}{3} \Lambda R^{2}+(1+2 E) \exp \left(-2 \int \mathrm{d} t \frac{p^{\prime}}{(\rho+p)} \frac{\dot{R}}{R^{\prime}}\right)-1 .
$$

Si en una dada región el gradiente de presión es grande, la tasa de expansión, $\mathrm{e}^{-A} \dot{R}^{2}$, disminuye. Luego, las superficies caracterizadas por $r=$ cte con valores mayores de $r$ se expanderán relativamente más rápido y el gradiente de presión disminuirá, dando lugar a un cambio de signo en $p^{\prime}$, y a un subsecuente incremento en la tasa expansión. Este comportamiento da origen a oscilaciones en los perfiles de $p^{\prime}$ y $\dot{R}$, como mostramos en la figura 2.1. Si las oscilaciones crecen lo suficiente, $\dot{R}$ puede cambiar de signo y dar lugar a un escenario de colapso [151]. Esta característica de la evolución de la geometría es consecuencia directa del gradiente de presión no nulo que caracteriza a las soluciones de Lemaître (notemos que el caso particular con $p^{\prime}=0$, las funciones métricas quedan reducidas a la forma $A(t, r)=0$ y $B(t, r)=\frac{R^{\prime}(t, r)}{(1+2 E(r))}$, es decir, que recuperamos la solución de LTB).

\subsection{La solución de Székeres}

La solución de Székeres (1975) [110] ha sido estudiada en el marco de modelos cosmológicos no homogéneos para describir la formación y evolución de cúmulos de galaxias y voids, 

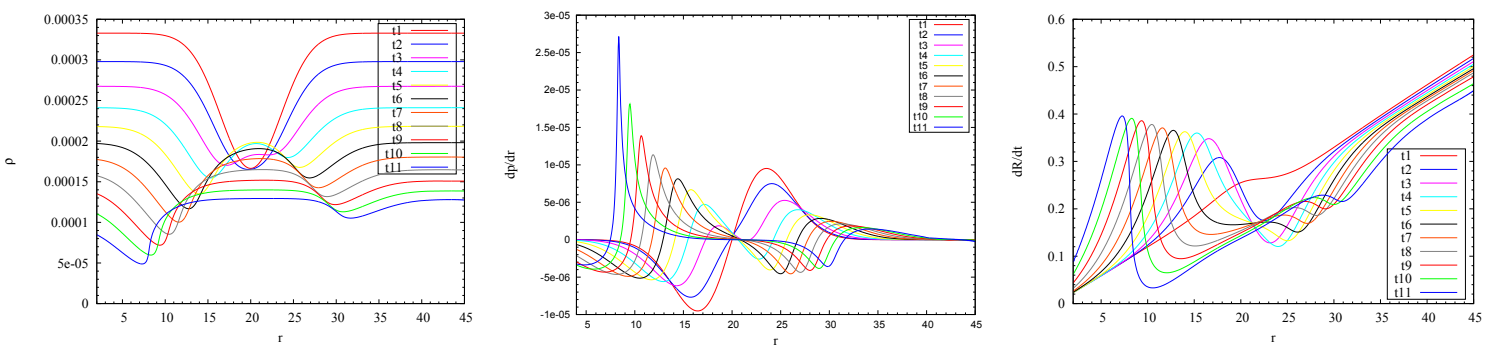

Figura 2.1: Evolución característica de la geometría de Lemaître para los perfiles de densidad, gradiente de presión y derivada temporal de la función $R$, respectivamente. El gradiente de presión no nulo en la ecuación de evolución para $R$ da origen a oscilaciones que pueden llevar a un escenario de colapso. Todas las curvas son de autoría propia, y fueron computadas mediante el algoritmo numérico detallado en el capítulo 4.

así como también para estudiar los efectos producidos por una geometría no homogénea sobre la propagación de los fotones provenientes del CMB en su pasaje a través de grandes estructuras. Presentaremos a continuación una breve introducción a esta solución y sus propiedades generales. Una descripción más completa puede ser encontrada en las referencias [16, 109, 137, 152].

La métrica de Székeres es descripta por el siguiente elemento de línea [109]

$$
\mathrm{d} s^{2}=\mathrm{d} t^{2}-\mathrm{e}^{2 \alpha} \mathrm{d} r^{2}-\mathrm{e}^{2 \beta}\left(\mathrm{d} x^{2}+\mathrm{d} y^{2}\right),
$$

en donde $\alpha=\alpha(t, x, y, r)$ y $\beta=\beta(t, x, y, r)$ son funciones a determinar a partir de las ecuaciones de Einstein con una fuente de fluido sin presión (esto es, polvo). Dado que las coordenadas $(t, x, y, r)$ son comóviles, la velocidad resulta $u^{\mu}=\delta_{0}^{\mu}$.

Existen dos familias de soluciones de Székeres, según sea $\beta^{\prime}=0$ o $\beta^{\prime} \neq 0$. El primer caso corresponde a una familia de soluciones que son una generalización simultánea de las soluciones de Friedmann-Lemaître y Kantowski-Sachs (1966) [4], y no posee una aplicación directa en problemas cosmológicos ni astrofísicos. Nos limitaremos entonces a presentar la familia de soluciones con $\beta^{\prime} \neq 0$.

A partir de las ecuaciones de Einstein para la métrica (2.50), tenemos [109]

$$
\begin{aligned}
\mathrm{e}^{\beta} & =\Phi(t, r) \mathrm{e}^{\nu(r, x, y)}, \\
\mathrm{e}^{\alpha} & =h(r) \Phi(t, r) \beta^{\prime} \equiv h(r)\left(\Phi^{\prime}+\Phi \nu^{\prime}\right), \\
\mathrm{e}^{-\nu} & =A(r)\left(x^{2}+y^{2}\right)+2 B_{1}(r) x+2 B_{2}(r) y+C(r),
\end{aligned}
$$

en donde la función $\Phi(t, r)$ es la solución de la ecuación

$$
\dot{\Phi}^{2}=-k(r)=\frac{2 M(r)}{\Phi}+\frac{1}{3} \Lambda \Phi^{2},
$$

con $h(r), k(r), M(r), A(r), B_{1}(r), B_{2}(r)$ y $C(r)$ funciones arbitrarias que obedecen la relación

$$
g(r) \equiv 4\left(A C-B_{1}^{2}-B_{2}^{2}\right)=1 / h^{2}(r)+k(r),
$$

en donde el término $1 / h^{2}(r)$ puede ser nulo sólo en puntos aislados (singularidades de coordenadas o cuellos). La solución de Székeres no presenta en general simetrías, pero para el 
caso en el que $A, B_{1}, B_{2}$ y $C$ son constantes (esto es, si $\nu^{\prime}=0$ ) adquiere simetría de grupo 3 -dimensional con órbitas 2-dimensionales. La densidad de masa en unidades de energía es

$$
\kappa \rho=\frac{\left(2 M \mathrm{e}^{3 \nu}\right)^{\prime}}{\mathrm{e}^{2 \beta}\left(\mathrm{e}^{\beta}\right)^{\prime}}, \quad\left(\kappa=8 \pi G / c^{4}\right) .
$$

La llamada función de bang-time, $t_{B}(r)$, es obtenida a partir de la siguiente integral

$$
\int_{0}^{\Phi} \frac{\mathrm{d} \tilde{\Phi}}{\sqrt{-k+2 M / \tilde{\Phi}+\frac{1}{3} \Lambda \tilde{\Phi}^{2}}}=t-t_{B}(r) .
$$

El signo de la función $g(r)$ determina la geometría de las 2-superficies con $t=$ cte y $r=$ cte. La geometría de estas superficies es esférica, plana o hiperbólica (pseudo-esférica) cuando $g>0, g=0$ o $g<0$, respectivamente. Siendo $A, B_{1}, B_{2}$ y $C$ funciones de $r$, las superficies con $r=$ cte y con $t=$ cte pueden tener direfentes geometrías; por ejemplo, pueden ser esferas en una parte del espacio y superficies con curvatura negativa constante en cualquier otro lugar, siendo la curvatura nula en el borde que separa ambas regiones.

El signo de la función $k(r)$ determina el tipo de evolución: con $k>0=\Lambda$, el modelo se expande desde una singularidad inicial, para colapsar luego en otra singularidad final; con $k<0=\Lambda$, el modelo presenta o bien expansión eterna, o bien colapso eterno, dependiendo de las condiciones iniciales dadas; el valor $k=0$ es un caso intermedio que corresponde al modelo de Friedmann-Lemaître plano ( $k=0$ puede también tener lugar en 3 -superficies como límite entre una región con $k<0$ y otra con $k>0$ ). Por su parte, el signo de $k(r)$ influencia el signo de $g(r)$, a través de la ecuación (2.53). Dado que el término $1 / h^{2}$ es no negativo, tenemos que: con $g>0$ (geometría esférica), todas las evoluciones son permitidas; con $g=0$ (geometría plana), $k$ debe ser no positivo (sólo evoluciones parabólica o hiperbólica son permitidas); y con $g<0$, (geometría hiperbólica), $k$ debe ser estrictamente negativo (sólo la evolución hiperbólica está permitida).

El límite de Friedmann-Lemaître se recupera cuando $\Phi(t, r)=r R(t), k=k_{0} r^{2}$ con $k_{0}=$ cte, y $B_{1}=B_{2}=0, C=4 A=1$. Esta definición del límite de Friedmann-Lemaitre incluye la definición del límite de coordenada radial (al igual que la métrica de LTB, la solución de Székeres es covariante ante transformaciones de la forma $r=f\left(r^{\prime}\right)$, con $f\left(r^{\prime}\right)$ una función arbitraria).

A partir del signo de la función $g(r)$ los modelos de Székeres son subdivididos en tres tipos: cuasiesféricos $(g>0)$, cuasiplanos $(g=0)$ y cuasihiperbólicos $(g<0)$. De estos, sólo el primero ha sido estudiado y aplicado a problemas cosmológicos. ${ }^{9}$ El modelo cuasiesférico puede ser imaginado como una generalización de los modelos de LTB, en los cuales las esferas de masa constante son no concéntricas. Las funciones $A, B_{1}$ y $B_{2}$ determinan cómo cambia de posición el centro una esfera en hiperespacios con $t=$ cte cuando el radio de la esfera crece o decrece. Más allá de esto, resulta una geometría simple porque todas las funciones arbitrarias dependen sólo de la coordenada $r$. Por su parte, los espacio-tiempos cuasiesféricos pueden ser pegados con la solución de Schwarschild a través de hipersuperficies con $t=$ cte [154].

Para el caso con $\Lambda=0$, las soluciones de la ecuación (2.52) involucran funciones elípticas [155]. Cualquier solución de (2.52), por otra parte, contiene una función arbitraria de integración adicional, $t_{B}(r)$, que al igual que en la solución de LTB aparece en las soluciones como $\left(t-t_{B}(r)\right)$. El instante $t=t_{B}(r)$ define el momento inicial de evolución: cuando $\Lambda=0$,

\footnotetext{
${ }^{9}$ Una interpretación de los modelos cuasiplanos y cuasihiperbólicos está presentada en las citas [137, 153].
} 
este instante es una singularidad en las ecuaciones y corresponde necesariamente a $\Phi=0$ (es el instante del big-bang en el límite de Friedmann-Lemaître). Cuando $t_{B}(r) \neq 0$ (esto es, en general) la singularidad inicial es dependiente de la posición, como en las soluciones de LTB.

Otra singularidad también puede ocurrir cuando $\left(\mathrm{e}^{\beta}\right)^{\prime}=0$, correspondiente a una región con shell crossing. Sin embargo, es cualitativamente diferente de aquella presente en las soluciones de LTB, dado que no necesariamente toda la superficie espacial colapsa. De las ecuaciones $(2,51)$, vemos que la condición $\left(\mathrm{e}^{\beta}\right)^{\prime}=0$ puede definir un subconjunto de superficies $\{x, y\}$ para cada $r$. Luego, la región de shell crossing para cada hipersuperficie 2-dimensional con $t=$ cte y $r=$ cte puede ser un círculo o, excepcionalmente, un punto.

La ecuación (2.52), por otra parte, es formalmente idéntica a la ecuación de FriedmannLemaître, pero con las funciones $k$ y $M$ dependientes de la coordenada $r$, con lo cual cada hipersuperficie con $r=$ cte evoluciona independiente de las otras. Las soluciones para $\Phi$ son las mismas que aquellas dadas por las ecuaciones (2.4)-(2.6), y no son afectadas por la dependencia con las coordenadas $\{x, y\}$ que caracterizan a las soluciones de Székeres.

Una manera más práctica de parametrizar las funciones arbitrarias en la métrica de Székeres es escribiendo el elemento de línea como [156].

$$
\mathrm{d} s^{2}=\mathrm{d} t^{2}-\frac{\left(\Phi^{\prime}-\Phi \mathcal{E}^{\prime} / \mathcal{E}\right)^{2}}{\epsilon-k(r)} \mathrm{d} r^{2}-\frac{\Phi^{2}}{\mathcal{E}^{2}}\left(\mathrm{~d} x^{2}+\mathrm{d} y^{2}\right)
$$

en donde

$$
\mathcal{E} \equiv \frac{S}{2}\left[\left(\frac{x-P}{S}\right)^{2}+\left(\frac{y-Q}{S}\right)^{2}+\epsilon\right],
$$

$\operatorname{con} g \neq 0, \epsilon \equiv g /|g|$ y $\mathrm{e}^{-\nu}=\sqrt{|g|} \mathcal{E}$, y las funciones arbitrarias dadas por $(A \neq 0)^{10}$

$$
\begin{array}{ll}
A=\frac{\sqrt{|g|}}{2 S}, \quad B_{1}=-\frac{\sqrt{|g|} P}{2 S}, & B_{2}=-\frac{\sqrt{|g|} Q}{2 S}, \\
C=\frac{\sqrt{|g|}}{2}\left[\frac{\left(P^{2}+Q^{2}\right)}{S}+\epsilon S\right], & h^{2}=1 /[|g|(\epsilon-k)] .
\end{array}
$$

El modelo cuasiesférico corresponde a la elección $\epsilon=+1$, mientras que el caso $g=0$ describe el modelo cuasiplano. La densidad de materia en estas variables es

$$
\kappa \rho=\frac{2\left(M^{\prime}-3 M \mathcal{E}^{\prime} / \mathcal{E}\right)}{\Phi^{2}\left(\Phi^{\prime}-\Phi \mathcal{E}^{\prime} / \mathcal{E}\right)}
$$

Definidos por las ecuaciones (2.51) y (2.52), los modelos de Székeres contienen 8 funciones de la coordenada $r$, de las cuales sólo 7 son arbitrarias debido a la restricción dada por la ecuación (2.53). Sin embargo, el número de funciones correspondiente a los grados de libertad físicos se reduce a 5 si tenemos en cuenta la elección de la coordenada radial (como, por ejemplo, $\tilde{r}=M(r)$ ), y que la parametrización (2.56 transforma la función $g(r)$ en el parámetro constante $\epsilon$.

Cabe mencionar que las definiciones anteriores son no covariantes. Existen, sin embargo, definiciones basadas en propiedades invariantes, pero resultan más complejas para trabajar y las omitiremos en esta introducción a las soluciones de Székeres [16].

\footnotetext{
${ }^{10}$ La elección $A \neq 0$ no pierde generalidad, dado que aún siendo $A=0$ inicialmente, un cambio de coordenadas $(x, y)$ puede dar lugar a $A \neq 0[16]$.
} 
Los modelos de Székeres tienen como fuente un fluido perfecto sin rotación. La aceleración es nula, $u_{; \beta}^{\alpha} u^{\beta}=0$, y el tensor de shear y el escalar de expansión resultan, respectivamente,

$$
\begin{aligned}
\sigma_{\beta}^{\alpha} & =\frac{1}{3}\left(\frac{\dot{\Phi}^{\prime}-\dot{\Phi} \Phi^{\prime} / \Phi}{\Phi^{\prime}-\Phi \mathcal{E}^{\prime} / \mathcal{E}}\right) \operatorname{diag}(0,2,-1,-1), \\
\Theta & =\frac{\dot{\Phi}^{\prime}+2 \dot{\Phi} \Phi^{\prime} / \Phi-3 \dot{\Phi} \mathcal{E}^{\prime} / \mathcal{E}}{\Phi^{\prime}-\Phi \mathcal{E}^{\prime} / \mathcal{E}}
\end{aligned}
$$

Notemos que en el límite de Friedmann-Lemaître, $R \rightarrow r a(t)$, se recuperan $\Theta \rightarrow 3 H$ y $\sigma_{\beta}^{\alpha} \rightarrow 0$. La ecuación de movimiento $T_{; \beta}^{\alpha \beta}=0$ se reduce a la ecuación de continuidad dada por

$$
\dot{\rho}+\rho \Theta=0 .
$$

El tensor de Weyl, por otro lado, puede ser descompuesto en sus partes eléctrica y magnética, definidas de la siguiente forma

$$
\begin{aligned}
E_{\beta}^{\alpha} & =C_{\gamma \beta \delta}^{\alpha} u^{\gamma} u^{\delta}=\frac{M\left(3 \Phi^{\prime}-\Phi M^{\prime} / M\right)}{3 \Phi^{3}\left(\Phi^{\prime}-\Phi \mathcal{E}^{\prime} / \mathcal{E}\right)} \operatorname{diag}(0,2,-1,-1) \\
H_{\alpha \beta} & =\frac{1}{2} \sqrt{-g} \epsilon_{\alpha \gamma \mu \nu} C_{\beta \delta}^{\mu \nu} u^{\gamma} u^{\delta}=0
\end{aligned}
$$

y los escalares de Ricci 4-dimensional y 3-dimensional resultan

$$
\begin{aligned}
{ }^{4} R & =-4 \Lambda-8 \pi G \rho, \\
{ }^{3} R & =2 \frac{k}{\Phi^{2}}\left(\frac{\Phi k^{\prime} / k-2 \Phi \mathcal{E}^{\prime} / \mathcal{E}}{\Phi^{\prime}-\Phi \mathcal{E}^{\prime} / \mathcal{E}}+1\right) .
\end{aligned}
$$

Una introducción a soluciones no homogéneas más generales puede encontrarse en las referencias [109] y [16] (capítulo 18). 
"Lo que él veía no era sólo el mensaje que el cielo le enviaba, sino el resultado de una amistad entre el cielo, la tierra y la posición (y la hora, y la estación, y el ángulo) desde la cual él miraba. A buen seguro, si el navío hubiera echado anclas a lo largo de otra diagonal de la rosa de los vientos, el espectáculo habría sido diferente, el sol, la aurora, el mar y la

tierra habrían sido otro sol, otra aurora, un mar y una tierra gemelos pero disformes.

Aquella infinidad de los mundos de la que le hablaba Saint-Savin no había que buscarla solamente allende las constelaciones, sino en el centro mismo de aquella burbuja del espacio de la cual él, puro ojo, era ahora origen de infinitas paralajes. (...)"

La isla del día de antes (1994)

Umberto Eco 


\section{Capítulo 3}

\section{Cosmología a tiempo real: la variación temporal del redshift cosmológico}

El avance de las técnicas observacionales en los últimos años ha dado lugar a la posibilidad de medir con alta precisión variaciones temporales de diferentes cantidades astrofísicas, en el contexto de la llamada cosmología a tiempo real o de alta precisión. Estas perspectivas impulsaron el estudio de observables cosmológicos que puedan servir para distinguir modelos de forma simple, y someter a prueba las hipótesis de homogeneidad e isotropía.

Entre estos observables, especial atención merece el llamado redshift drift, definido como la variación temporal del redshift cosmológico debida a la tasa variable de expansión del universo. El redshift drift ofrece una medida directa de la aceleración en la expansión cósmica, y está fuertemente relacionado con el mecanismo físico que domina la dinámica del universo. Su potencial para distinguir cosmologías radica en que las predicciones teóricas de esta cantidad para distintos modelos muestran marcadas diferencias, tanto cualitativas como cuantitativas. En particular, para el caso del modelo $\Lambda \mathrm{CDM}$ la predicción teórica del redshift drift indica valores positivos para fuentes cercanas, mientras que para el caso de modelos no homogéneos su signo puede depender del perfil radial de densidad, así como también de la simetría del problema y de la dirección de llegada de los fotones.

En este contexto, presentaremos en este capítulo un estudio del redshift drift de fuentes cercanas, a partir de dos tratamientos independientes. En primer lugar, abordaremos el trabajo a partir de una formulación covariante general, basada en el formalismo $1+3$. Este enfoque es esencialmente simple y ofrece información relevante sobre el valor esperable del redshift drift para fuentes cercanas, con la ventaja de prescindir de la integración de geodésicas nulas. La aplicación de esta formulación covariante es amplia y, en el contexto de esta tesis, mostraremos algunos resultados interesantes para el caso de modelos cosmológicos basados en soluciones no homogéneas de las ecuaciones de Einstein en Relatividad General.

Por otra parte, también motivados en poder distinguir modelos cosmológicos, presentaremos un tratamiento cosmográfico para estudiar el redshift drift de fuentes cercanas a partir de parámetros cosmológicos cinemáticos. Este enfoque presenta la ventaja de ser independiente de la dinámica supuesta en un dado modelo. Como ejemplo de aplicación del tratamiento cosmográfico, presentaremos en el apéndice E un trabajo complementario que desarrollamos en el marco de modelos cosmológicos basados en teorías de gravedad modificada $f(R)$. 


\subsection{Cosmología a tiempo real}

El avance de las técnicas observacionales astrométricas y espectroscópicas en los últimos años ha dado lugar a la posibilidad de medir con alta precisión variaciones temporales de cantidades astrofísicas observables dentro de un intervalo de tiempo relativamente pequeño. Estas perspectivas impulsaron el estudio de diferentes observables cosmológicos en el marco de lo que se conoce como cosmología a tiempo real o de alta precisión [139].

Las observaciones a tiempo real pueden estar asociadas a variaciones temporales en las posiciones y/o velocidades de diferentes fuentes astronómicas. Dichas variaciones pueden clasificarse en dos grupos: (i) las que son consecuencia de la evolución cosmológica, y (ii) aquellas originadas por movimientos peculiares de las fuentes, tanto en la dirección radial como la dirección transversal respecto a la línea de la visual del observador.

Dentro del primer grupo podemos mencionar, en primer lugar, la variación temporal del redshift de fuentes lejanas debida a la tasa de expansión variable del universo, conocida como redshift drift [157, 158]. Esta cantidad puede ser utilizada como trazador de la expansión cosmológica en la dirección radial. Este observable representa una medida directa de la variación del parámetro de Hubble, $H(z)$, con respecto a su valor actual, y podría proporcionar información sobre los mecanismos físicos que dan lugar a la aceleración de la expansión del universo [88].

La variación temporal de la separación angular de dos fuentes distantes (por ejemplo, quásares) se conoce como paralaje cósmica [159]. Es, en otras palabras, la cantidad análoga al redshift drift pero en la dirección transversal a la línea de la visual. En modelos homogéneos e isótropos, la separación angular entre dos fuentes es constante (excepto por los efectos debidos a movimientos peculiares de la fuente) y la paralaje cósmica resulta nula, mientras que en modelos con geometrías más complejas su valor es distinto de cero. Esta cantidad representa entonces un test de anisotropía, ya sea por propiedades intrínsecas del modelo (como por ejemplo, el caso de la métrica de Bianchi (ver apéndice C)), o debida a la posición del observador fuera del centro de simetría en modelos con simetría esférica (como el caso de modelos de void basados en la solución de $\operatorname{LTB}^{1}$ ) [88, 160, 161].

Entre las variaciones originadas por movimientos propios de las fuentes, podemos mencionar la llamada aceleración peculiar [162]. Esta cantidad está asociada a la medición del redshift drift de fuentes cercanas $(z \lesssim 0,005)$, para las cuales se espera que la señal debida a la expansión cósmica sea despreciable. Es decir, es un trazador de la variación de velocidades peculiares de objetos en cúmulos y galaxias, causada por los potenciales gravitacionales de las estructuras que los contienen. La medición de la aceleración peculiar de objetos cercanos permitiría entonces distinguir entre diferentes modelos de gravedad (por ejemplo, Relatividad General vs. teorías $f(R)$, gravedad newtoniana vs. MOND [163]). En forma análoga, si la señal de la paralaje cósmica es dominada por movimientos peculiares en la dirección transversal a la línea de la visual, la variación temporal de la separación angular entre dos fuentes se asocia a lo que se conoce como aceleración propia [139].

Entre los observables cosmológicos mencionados anteriormente, nos abocaremos en lo que resta de este capítulo a estudiar el redshift drift, como herramienta para distinguir diferentes modelos cosmológicos.

\footnotetext{
${ }^{1}$ En este caso la paralaje cósmica es también un test para el Principio Copernicano [139].
} 


\subsubsection{Redshift drift como test para distinguir modelos cosmológicos}

La variación del redshift cosmológico de las galaxias, conocida en la literatura como redshift drift o efecto Sandage [157, 158, 164], es un observable que puede ser utilizado para estudiar la aceleración de la expansión del universo. Si bien fue durante años considerado apenas como un test "ideal" debido a limitaciones observacionales, en el contexto de la cosmología de alta precisión se espera poder obtener mediciones confiables de este efecto dentro de los próximos 20 años $[139,165]$. Ante esta perspectiva positiva, resulta de gran interés estudiar las predicciones teóricas del redshift drift de galaxias en modelos cosmológicos no homogéneos, con el fin de hacer futuras comparaciones y poder poner restricciones sobre distintos modelos cosmológicos. La medida de la tasa de expansión cósmica a diferentes redshifts es, por otra parte, de extrema importancia para investigar el mecanismo físico que daría origen a la expansión acelerada del universo $[160,166]$.

Los proyectos observacionales inminentes dieron lugar a profundizar el estudio de las predicciones teóricas para el redshift drift en diferentes escenarios cosmológicos (e.g., modelos de energía oscura $[167,168,169,170,171,172]$, modelos basados en soluciones no homogéneas $[88,173,174,175,176,177,178]$, y modelos estudiados dentro del tratamiento de promedios o backreaction [179], entre otros). Los resultados de estos trabajos muestran que, dependiendo de la geometría y las características intrínsecas de cada modelo, se espera que la curva del redshift drift en función de $z$ exhiba trazos distinguibles que puedan utilizarse para discriminar modelos cosmológicos [88], así como también como un test de validez del Principio Cosmológico [166]. Un breve resumen de las predicciones teóricas para el redshift drift en diferentes modelos está presentada en el apéndice D.

En particular, cabe hacer especial mención a un resultado interesante presentado en [180] acerca del redshift drift calculado para el caso de modelos de void basados en la solución de LTB. Los resultados de dicho trabajo muestran que la estimación del redshift drift para fuentes cercanas en un modelo de void arroja valores con signo definido negativo, mientras que para modelos homogéneos basados en la solución de FLRW el redshift drift resulta siempre positivo. Esta importante diferencia indica que el redshift dift podría ser utilizado para distinguir modelos cosmológicos simplemente a partir de la determinación de su signo.

Motivados por estos resultados, nos propusimos estudiar el redshift drift en diferentes modelos cosmológicos, con el fin de encontrar trazos característicos que los distingan. Dado que el cálculo exacto del redshift drift en geometrías con pocas simetrías (o ninguna) es, en general, complejo (ver apéndice D) trabajaremos con aproximaciones analíticas válidas para fuentes cercanas. A partir de las mismas, es posible inferir información sobre los modelos cosmológicos sin necesidad de integrar la ecuación de las geodésicas nulas que siguen los fotones.

Presentaremos dos tratamientos para calcular el redshift drift de fuentes cercanas. El primero es una formulación covariante basada en el formalismo de descomposición $1+3$ [5]. La ventaja de este enfoque es que el redshift drift queda expresado en términos de cantidades cinemáticas escalares que dependen únicamente de las funciones métricas y pueden ser calculados fácilmente, incluso en geometrías complejas, como aquellas descriptas por soluciones no homogéneas de la Relatividad General.

La segunda aproximación se basa en un tratamiento cosmográfico del redshift drift. Este enfoque puede ser aplicado sólo a modelos basados en la métrica de FLRW, pero presenta la ventaja de ser independiente de la dinámica que domina la evolución del factor de escala en el universo. Es decir, que puede ser utilizado para estudiar modelos cosmológicos basados 
tanto en teorías alternativas a la Relatividad General, como en teorías de gravedad modificada $f(R)$. En esta dirección, hemos utilizado nuestra expresión cosmográfica del redshift drift para encontrar restricciones sobre el espacio de parámetros de algunos modelos cosmológicos en el marco de teorías $f(R)$. Este trabajo, complementario al que fue desarrollado en la tesis, está presentado en el apéndice E.

\subsection{Formulación covariante del redshift drift $(z \ll 1)$}

La formulación covariante que presentaremos en esta sección tiene como objetivo expresar el redshift drift de fuentes cercanas en términos los parámetros cinemáticos de una geometría dada, las cuales pueden ser calculados en forma sencilla, aún en el caso de geometrías complejas. Para ello, trabajaremos en el formalismo covariante de la descomposición $1+3$ [5, 181]. La ventaja de trabajar con este formalismo es que tanto la física cuanto la geometría pueden ser descriptas mediante cantidades y relaciones tensoriales que permanecen válidas en cualquier sistema de referencia con el que se elija trabajar. Los detalles de la descomposición $1+3$, así como algunas definiciones básicas que utilizaremos para nuestro cálculo del redshift drift, están presentadas en el apéndice A.

El redshift cosmológico de un fotón emitido por una dada fuente y medido en un punto $\mathcal{O}$ se define como [16]

$$
z \equiv \frac{\nu_{e}}{\nu_{o}}-1=\frac{\left(k_{\alpha} u^{\alpha}\right)_{e}}{\left(k_{\alpha} u^{\alpha}\right)_{o}}-1
$$

en donde los subíndices $e$ y $o$ indican, respectivamente, la frecuencia de los fotones emitidos por la fuente y la de los fotones recibidos en un punto $\mathcal{O}$, y $u^{\alpha}$ es la velocidad del sistema de referencia. Un rayo de luz con vector de onda $k^{\alpha}$ incide en la dirección determinada por el vector unitario $n^{\alpha}\left(\operatorname{con} n_{\alpha} n^{\alpha}=-1\right)$, que resulta colineal a la proyección de $k^{\alpha}$ sobre las hipersuperficies con $t$ constante. Luego, se tiene

$$
n^{\alpha}=\frac{1}{\left(k_{\gamma} u^{\gamma}\right)}\left(\delta_{\beta}^{\alpha}-u^{\alpha} u_{\beta}\right) k^{\beta}=-\frac{k^{\alpha}}{k_{\gamma} u^{\gamma}}+u^{\alpha},
$$

en donde el signo negativo indica que el vector $n^{\alpha}$ está orientado en el sentido opuesto al de incidencia del fotón. El vector $n^{\alpha}$ resulta entonces perpendicular a la velocidad $u^{\alpha}$ (esto es, $\left.n^{\alpha} u_{\alpha}=0\right)$.

Hasta aquí no hemos hecho ninguna restricción sobre la distancia que separa la fuente del punto $\mathcal{O}$. Ahora bien, para calcular el redshift cosmológico de una fuente arbitraria es necesario integrar la expresión (3.1) a lo largo de la geodésica nula de los fotones, tarea que resulta no trivial para geometrías con poca simetría. Sin embargo, una aproximación útil puede adoptarse para el caso de fuentes cercanas. Si $z \ll 1$, resulta

$$
z=\frac{\left(k_{\alpha} u^{\alpha}\right)_{e}-\left(k_{\alpha} u^{\alpha}\right)_{o}}{\left(k_{\alpha} u^{\alpha}\right)_{o}} \simeq \frac{\mathrm{d}\left(k_{\alpha} u^{\alpha}\right)}{\left(k_{\alpha} u^{\alpha}\right)_{o}}
$$

en donde la derivada $\mathrm{d}(\cdot)$ debe ser calculada a lo largo de un rayo de luz que conecte la fuente con el punto $\mathcal{O}$, es decir, $\mathrm{d}\left(A_{\nu}\right)=\dot{A_{\alpha}} \equiv\left(k_{\beta} \nabla^{\beta}\right) A_{\alpha}$. Si llamamos $v$ al parámetro afín de la geodésica del fotón medido, obtenemos que

$$
\mathrm{d}\left(k_{\alpha} u^{\alpha}\right)=\left(k_{\alpha} u^{\alpha}\right)=\left(k_{\alpha ; \beta} k^{\beta} u^{\alpha}\right)_{o} \mathrm{~d} v+\left(k_{\alpha} u_{; \beta}^{\alpha} k^{\beta}\right)_{o} \mathrm{~d} v .
$$


Teniendo en cuenta que $k_{\beta ; \alpha} k^{\alpha}=0$, y reemplazando la derivada covariante de la velocidad $u_{\alpha}=\delta_{\alpha}^{0}$ por su descomposición en las cantidades cinemáticas (ecuación (A.11) del apéndice A), resulta

$$
\begin{aligned}
\mathrm{d}\left(k_{\alpha} u^{\alpha}\right) & =\left[\left(\sigma_{\alpha \beta} k^{\alpha} k^{\beta}\right)_{o}-\frac{1}{3} \Theta_{o}\left(k_{\alpha} u^{\alpha}\right)_{o}^{2}+\left(k_{\alpha} \dot{u}^{\alpha}\right)_{o}\left(k_{\alpha} \dot{u}^{\alpha}\right)_{o}\right] \mathrm{d} v \\
& =\left(k_{\gamma} u^{\gamma}\right)_{o}^{2}\left(\sigma_{\alpha \beta} n^{\alpha} n^{\beta}-\frac{1}{3} \Theta-n_{\alpha} \dot{u}^{\alpha}\right)_{o} \mathrm{~d} v .
\end{aligned}
$$

Por otra parte, la cantidad $\delta \ell_{o} \equiv-\left(k_{\gamma} n^{\gamma}\right)_{o} \mathrm{~d} v$ es la distancia entre la fuente y el punto $\mathcal{O}$, correspondiente a una variación $\mathrm{d} v$ del parámetro afín. Luego, la expresión final de la aproximación del redshift de fuentes cercanas a $\mathcal{O}$, medido a un tiempo $t=t_{o}$, resulta [182]

$$
z_{o} \simeq\left(\frac{1}{3} \Theta-\sigma_{\alpha \beta} n^{\alpha} n^{\beta}+n_{\alpha} \dot{u}^{\alpha}\right)_{o} \delta \ell_{o}
$$

La expresión (3.6) es independiente de la teoría gravitacional con la que estamos trabajando, y ofrece la ventaja de poder calcular el redshift de fuentes cercanas sin necesidad de integrar las ecuaciones diferenciales correspondientes a las geodésicas de los fotones. Por otra parte, podemos notar que para fuentes cercanas la rotación (dada por el vector de vórtice $\omega^{\alpha} \equiv$ $\left.\frac{1}{2} \eta^{\alpha \beta \gamma} \omega_{\beta \gamma}\right)$ no influye sobre el cálculo del redshift, mientras que la contribución anisótropa proviene de los términos con $\sigma_{\alpha \beta}$ y $\dot{u}^{\alpha}$.

Utilizaremos la expresión anterior para calcular el redshift drift de una dada fuente en forma covariante. El redshift de la fuente medido en el punto $\mathcal{O}$, a un tiempo $t_{o^{\prime}}=t_{o}+\delta t_{o}$, se calcula como

$$
z_{O^{\prime}} \simeq\left(\frac{1}{3} \Theta-\sigma_{\alpha \beta} n^{\alpha} n^{\beta}+n_{\alpha} \dot{u}^{\alpha}\right)_{o^{\prime}} \delta \ell_{o^{\prime}} .
$$

Consideraremos, en adelante, que $\dot{u}^{\alpha} \equiv 0$. La variación del redshift cosmológico de la fuente puede calcularse entonces como $\delta z=z_{o^{\prime}}-z_{o}$. Teniendo en cuenta que $\delta t_{o} / t_{o} \ll 1$, podemos aproximar $z_{o^{\prime}} \simeq z_{o}+\dot{z}_{o} \delta t_{o}$. Luego, resulta

$$
\begin{aligned}
\delta z & =z_{O^{\prime}}-z_{o} \simeq\left[z_{o}+\left(\frac{\mathrm{d} z}{\mathrm{~d} t}\right)_{o} \delta t_{o}\right]-z_{o} \\
& =\left[\frac{\mathrm{d}}{\mathrm{d} t}\left(\frac{1}{3} \Theta-\sigma_{\alpha \beta} n^{\alpha} n^{\beta}\right)\right]_{o} \delta \ell_{o} \delta t_{o}+\left(\frac{1}{3} \Theta-\sigma_{\alpha \beta} n^{\alpha} n^{\beta}\right)_{o}\left(\frac{\mathrm{d}}{\mathrm{d} t} \delta \ell\right)_{o} \delta t_{o} \\
& =\left(\frac{1}{3} \frac{\mathrm{d} \Theta}{\mathrm{d} t}-\frac{\mathrm{d}}{\mathrm{d} t}\left(\sigma_{\alpha \beta} n^{\alpha} n^{\beta}\right)\right)_{o} \delta t_{o} \delta \ell_{o}+\left(\frac{1}{3} \Theta-\left(\sigma_{\alpha \beta} n^{\alpha} n^{\beta}\right)\right)_{o}\left(\frac{\mathrm{d}}{\mathrm{d} t} \delta \ell\right)_{o} \delta t_{o} \\
& =\left\{\left(\frac{1}{3} \frac{\mathrm{d} \Theta}{\mathrm{d} t}-\frac{\mathrm{d} \sigma_{\alpha \beta}}{\mathrm{d} t} n^{\alpha} n^{\beta}-\sigma_{\alpha \beta} \frac{\mathrm{d}}{\mathrm{d} t}\left(n^{\alpha} n^{\beta}\right)\right)_{o} \delta \ell_{o}+\left(\frac{1}{3} \Theta-\left(\sigma_{\alpha \beta} n^{\alpha} n^{\beta}\right)\right)_{o}\left(\frac{\mathrm{d}}{\mathrm{d} t} \delta \ell\right)_{o}\right\}_{o} \delta t_{o}, \\
\Rightarrow & \left(\frac{\delta z}{\delta t_{o}}\right)=\left[\frac{1}{3} \frac{\mathrm{d} \Theta}{\mathrm{d} t}-\frac{\mathrm{d} \sigma_{\alpha \beta}}{\mathrm{d} t} n^{\alpha} n^{\beta}-\sigma_{\alpha \beta} \frac{\mathrm{d}}{\mathrm{d} t}\left(n^{\alpha} n^{\beta}\right)\right]_{o} \delta \ell_{o}+\left[\frac{1}{3} \Theta-\left(\sigma_{\alpha \beta} n^{\alpha} n^{\beta}\right)\right]_{o}\left(\frac{\mathrm{d}}{\mathrm{d} t} \delta \ell\right)_{o} .
\end{aligned}
$$

Ahora bien, trabajemos con los primeros tres términos. Teniendo en cuenta que $u^{\alpha}=\delta_{0}^{\alpha}, \mathrm{y}$ que

$$
u^{\alpha} \nabla_{\alpha}\left(A_{\beta} B^{\beta}\right)=A_{\beta} u^{\alpha} \nabla_{\alpha} B^{\beta}+B^{\beta} u^{\alpha} \nabla_{\alpha} A_{\beta}=A_{\alpha} \frac{\mathrm{d} B^{\alpha}}{\mathrm{d} t}+B^{\alpha} \frac{\mathrm{d} A_{\alpha}}{\mathrm{d} t}
$$


los dos primeros pueden ser reemplazados por las ecuaciones de propagación (A.14) y (A.15), respectivamente. Por otra parte, la derivada en el término restante resulta

$$
\frac{\mathrm{d} n^{\alpha}}{\mathrm{d} t}=\left(u^{\beta} \nabla_{\beta}\right) n^{\alpha}=-\frac{u^{\beta} \nabla_{\beta} k^{\alpha}}{k_{\gamma} u^{\gamma}}+\frac{k^{\alpha} u_{\delta} u^{\beta} \nabla_{\beta} k^{\delta}}{\left(k_{\gamma} u^{\gamma}\right)^{2}} .
$$

Además, de la ecuación de evolución (A.12) [5], la derivada de la distancia entre la fuente y el punto $\mathcal{O}$ es

$$
\left.\frac{\mathrm{d} \delta \ell}{\mathrm{d} t}\right|_{o}=\left.\left(u^{\alpha} \nabla_{\alpha}\right) \delta \ell\right|_{o}=\left(\frac{1}{3} \Theta+\sigma_{\alpha \beta} n^{\alpha} n^{\beta}\right)_{o} \delta \ell_{o},
$$

en donde $\delta \ell_{o}$ se escribe en términos de $z$ y de las cantidades cinemáticas, a través de la ecuación (3.6), como

$$
\delta \ell_{o}=\frac{z}{\left(\frac{1}{3} \Theta-\sigma_{\alpha \beta} n^{\alpha} n^{\beta}\right)_{o}} .
$$

Finalmente, reemplazando todos los términos correspondientes en la ecuación (3.8), resulta

$$
\begin{aligned}
\frac{\delta z}{\delta t_{o}}=\left\{n_{\alpha} n_{\beta}\left(\frac{2}{3} \Theta \sigma^{\alpha \beta}+\sigma_{\gamma}^{<\alpha} \sigma^{\beta>\gamma}+\omega^{<\alpha} \omega^{\beta>}+E^{\alpha \beta}\right)-\left(\sigma_{\alpha \beta} n^{\alpha} n^{\beta}\right)^{2}\right. \\
+\frac{2 \sigma_{\alpha \beta} n^{\alpha}}{\left(k_{\gamma} u^{\gamma}\right)^{2}}\left(\left(k_{\gamma} u^{\gamma}\right) u^{\delta} \nabla_{\delta} k^{\beta}-k^{\beta} u_{\gamma} u^{\delta} \nabla_{\delta} k^{\gamma}\right) \\
\left.\quad-\frac{2}{3} \sigma^{2}+\frac{2}{3} \omega^{2}-\frac{1}{6}(\rho+3 p)+\frac{\Lambda}{3}\right\}_{o} \frac{z}{\left(\frac{1}{3} \Theta-\sigma_{\alpha \beta} n^{\alpha} n^{\beta}\right)_{o}}
\end{aligned}
$$

La ecuación (3.12) es la expresión covariante del redshift drift en términos de las cantidades cinemáticas que caracterizan al fluido cosmológico, $\Theta, \sigma_{\alpha \beta}$ y $\omega^{\alpha}$, y la parte eléctrica del tensor de Weyl, $E^{\alpha \beta}$, válida para fuentes cercanas al punto de medición $\mathcal{O}(z \ll 1)$ y aplicable sólo a problemas de la Teoría de la Relatividad General (las ecuaciones de propagación (A.15) y (A.14) dependen de la teoría gravitacional subyacente). El vector $k^{\alpha}$ es característico de cada geometría y debe ser calculado para el problema particular que se quiera estudiar. Dado que todas las cantidades están evaluadas en el punto de medición $\mathcal{O}$, no es necesario recurrir a la integración de las geodésicas nulas que, en el caso general de soluciones con menos simetrías, es compleja y debe realizarse en forma numérica (ver apéndice D). Asimismo, los efectos anisótropos son introducidos a través de las cantidades $\sigma^{\alpha \beta}, \omega^{\alpha}$ y $E^{\alpha \beta}$.

\subsubsection{Aplicaciones a diferentes escenarios cosmológicos}

A modo de ejemplo de aplicación, mostraremos a continuación el cálculo del redshift drift para algunas de las geometrías que estudiamos en esta tesis. Como veremos, en algunos casos dicho observable presenta un signo definido, lo cual evidencia su potencial para distinguir modelos cosmológicos.

\section{Modelo $\Lambda$ CDM:}

Comencemos por analizar el caso más simple, descripto por la métrica homogénea de FLRW y un fluido de materia con velocidad $u^{\alpha}=\delta_{0}^{\alpha}$. El elemento de línea es

$$
\mathrm{d} s^{2}=c^{2} \mathrm{~d} t^{2}-a^{2}(t)\left[\frac{\mathrm{d} r^{2}}{1-k r^{2}}+r^{2}\left(\mathrm{~d} \theta^{2}+\operatorname{sen}^{2} \theta \mathrm{d} \phi\right)\right] .
$$


El vector de onda de un fotón incidente en un punto $\mathcal{O}, k^{\alpha} \equiv \mathrm{d} x^{\alpha} / \mathrm{d} \lambda$, se calcula a partir de la ecuación de la geodésica, dada por

$$
\frac{\mathrm{d}^{2} x^{\alpha}}{\mathrm{d} \lambda^{2}}-\Gamma_{\beta \gamma}^{\alpha} \frac{\mathrm{d} x^{\beta}}{\mathrm{d} \lambda} \frac{\mathrm{d} x^{\gamma}}{\mathrm{d} \lambda}=0,
$$

y la condición de geodésica nula, $k^{\alpha} k_{\alpha}=0$. En este caso resulta

$$
k^{\mu}=\left[\frac{1}{a},-\frac{\sqrt{1+k r^{2} / 4}}{a}, 0,0\right] .
$$

Por otra parte, las cantidades cinemáticas para esta geometría son

$$
a^{\alpha}=\sigma^{\alpha \beta}=\omega^{\alpha \beta}=0 \quad \text { y } \quad \Theta=3 H=3 \frac{\dot{a}}{a},
$$

y luego, la expresión covariante del redshift drift para fuentes cercanas se reduce simplemente a

$$
\frac{\delta z}{\delta t_{o}}=\frac{1}{2} z H_{o}\left(2 \Omega_{\Lambda, o}-\Omega_{m, o}\right) .
$$

Es decir, que el redshift drift calculado para el modelo $\Lambda$ CDM resulta siempre positivo para fuentes cercanas $(z \ll 1)$. Este resultado coincide con aquel encontrado previamente por otros autores en los trabajos [88, 164] (ver apéndice D).

\section{Modelos de LTB:}

Estudiemos ahora el caso de un modelo cosmológico con un fluido no homogéneo, sin presión y con simetría esférica, descripto por la solución de LTB, con elemento de línea

$$
\mathrm{d} s^{2}=\mathrm{d} t^{2}-\frac{\left[R^{\prime}(t, r)\right]^{2}}{1+2 E(r)} \mathrm{d} r^{2}-R^{2}(t, r) \mathrm{d} \Omega^{2} .
$$

Para el caso de geodésicas radiales (esto es, cuando el punto de incidencia de los fotones $\mathcal{O}$ es el centro de simetría), el vector de onda resulta

$$
k^{\alpha}=\left(\frac{1}{R^{\prime}},-\frac{\sqrt{1+2 E}}{\left(R^{\prime}\right)^{2}}, 0,0\right),
$$

y las cantidades cinemáticas vienen dadas por

$$
a^{\alpha}=\sigma^{\alpha \beta}=\omega^{\alpha \beta}=0 \quad \text { y } \quad \Theta=\left(\dot{R}^{\prime}\right)_{o}=3 H_{o} .
$$

Luego, la expresión covariante del redshift drift para fuentes cercanas puede ser escrita de una forma simple, similar a la del caso anterior, como

$$
\frac{\delta z}{\delta t_{o}}=-\frac{1}{2} z H_{o} \Omega_{m, o} .
$$

Podemos notar entonces que para modelos de LTB el redshift drift de fuentes cercanas medido desde el centro de simetría es siempre negativo. Este resultado, junto con aquel obtenido para el caso de FLRW, evidencia claramente el potencial del redshift drift para distinguir modelos 
cosmológicos. Los signos definidos en cada uno de estos casos permite discriminar entre dichas cosmologías, aún disponiendo de una medida pequeña del efecto en cuestión (ver la discusión en la sección 3.4 sobre las perspectivas observacionales para el redshift drift).

El resultado anterior fue obtenido previamente en [180] a partir de las propiedades de las ecuaciones de las geodésicas nulas de los fotones, para un caso particular de modelos de void de LTB. ${ }^{2}$ La ecuación (3.21) es, sin embargo, válida para cualquier modelo de LTB y los cálculos involucrados a partir de la expresión (3.12) son notablemente menos complejos.

\section{Modelos cuasiesféricos de Székeres:}

Una aplicación interesante de la fórmula (3.12) se da en el caso de los modelos cuasiesféricos de Székeres. El redshift drift en este tipo de modelos fue estudiado a partir de la integración de las trayectorias geodésicas de los fotones, aunque sin considerar la dependencia angular con la dirección de incidencia. Los resultados muestran que, al igual que en los modelos de void de LTB, la predicción para el redshift drift arroja valores negativos para todo $z$ [183, 184] (ver apéndice D). Calcularemos aquí la expresión covariante para el caso de fuentes cercanas.

El elemento de línea que caracteriza la métrica viene dado por [16, 109]

$$
\mathrm{d} s^{2}=\mathrm{d} t^{2}-\frac{\left(\Phi^{\prime}-\Phi \mathcal{E}^{\prime} / \mathcal{E}\right)^{2}}{\epsilon-k(r)} \mathrm{d} r^{2}-\frac{\Phi^{2}}{\mathcal{E}^{2}}\left(\mathrm{~d} x^{2}+\mathrm{d} y^{2}\right)
$$

en donde $\Phi=\Phi(t, r), k=k(r), \epsilon=+1, \mathrm{y}$

$$
\mathcal{E} \equiv \frac{S}{2}\left[\left(\frac{x-P}{S}\right)^{2}+\left(\frac{y-Q}{S}\right)^{2}+\epsilon\right],
$$

con $S=S(r), P=P(r)$ y $Q=Q(r)$ funciones arbitrarias de la coordenada radial. Las ecuaciones de Einstein para el modelo quasi-esférico de Székeres se reducen a

$$
\dot{\Phi}=\frac{2 M}{\Phi}-k+\frac{1}{3} \Lambda \Phi^{2},
$$

en donde $\Lambda$ es la constante cosmológica y la función arbitraria $M(r)$ está relacionada con la densidad mediante la siguiente expresión

$$
\kappa \rho=\frac{2 M^{\prime}-6 M \mathcal{E}^{\prime} / \mathcal{E}}{\Phi^{2}\left(\Phi^{\prime}-\Phi \mathcal{E}^{\prime} / \mathcal{E}\right)}, \quad \kappa=8 \pi G / c^{4}, \quad c=1 .
$$

La integración de la ecuación (3.24) resulta

$$
\pm \int_{0}^{\Phi} \frac{\mathrm{d} \tilde{\Phi}}{\sqrt{-k+2 M / \tilde{\Phi}+\frac{1}{3} \Lambda \tilde{\Phi}^{2}}}=t-t_{B}(r)
$$

\footnotetext{
${ }^{2}$ Definiremos, en este contexto, un modelo de LTB de void como aquel que satisface las siguientes características [180]:

1. la densidad de masa $\rho$ es no negativa;

2. la densidad de masa $\rho$ es una función monótonamente creciente de $r$ sobre hipersuperficies con $t=$ cte;

3. $R^{\prime}$ es una cantidad positiva; $\mathrm{y}$

4. el modelo es $z$-normal (es decir, que $z$ es una función monótona de la coordenada $r$ ) .
} 
en donde la función arbitraria de integración $t_{B}(r)$, llamada usualmente bang-time, define el momento inicial de la evolución. Cuando $\left(t_{B}\right)^{\prime} \neq 0$, en general la "singularidad inicial" depende de la posición, como en los modelos de LTB. Los signos '+' y '-' describen, respectivamente, regiones de expansión y colapso.

Todas las ecuaciones anteriores son covariantes ante transformaciones de la forma $\tilde{r}=$ $g(r)$. Es decir, que una de las seis funciones arbitrarias $k(r), S(r), P(r), Q(r), M(r)$ o $t_{B}(r)$ pueden ser fijadas mediante una elección conveniente de $g(r)$. Con esto, el modelo queda completamente determinado por 5 funciones de $r$ y la elección de la coordenada radial. En particular, trabajaremos en este ejemplo con el caso $\Lambda=0$.

Las ecuaciones de las geodésicas para los fotones resultan [184]

$$
\begin{aligned}
& \frac{\mathrm{d}^{2} t}{\mathrm{~d} \lambda^{2}}+\frac{\Phi,{ }_{t r}-\Phi,{ }_{,} \mathcal{E}_{r} / \mathcal{E}}{1-k}\left(\Phi,_{r}-\Phi \mathcal{E},_{r} / \mathcal{E}\right)\left(\frac{\mathrm{d} r}{\mathrm{~d} \lambda}\right)^{2} \\
& +\frac{\Phi \Phi, t}{\mathcal{E}^{2}}\left[\left(\frac{\mathrm{d} x}{\mathrm{~d} \lambda}\right)^{2}+\left(\frac{\mathrm{d} y}{\mathrm{~d} \lambda}\right)^{2}\right]=0, \\
& \frac{\mathrm{d}^{2} r}{\mathrm{~d} \lambda^{2}}+2 \frac{\Phi,{ }_{t r}-\Phi,{ }_{,} \mathcal{E},_{r} / \mathcal{E}}{\Phi,{ }_{r}-\Phi \mathcal{E},{ }_{r} / \mathcal{E}} \frac{\mathrm{d} t}{\mathrm{~d} \lambda} \frac{\mathrm{d} r}{\mathrm{~d} \lambda}+\left(\frac{\Phi,_{r r}-\Phi \mathcal{E},_{r r} / \mathcal{E}}{\Phi_{,_{r}}-\Phi \mathcal{E},_{r} / \mathcal{E}}-\frac{\mathcal{E},_{r}}{\mathcal{E}}+\frac{1}{2} \frac{k,_{r}}{1-k}\right)\left(\frac{\mathrm{d} r}{\mathrm{~d} \lambda}\right)^{2}
\end{aligned}
$$

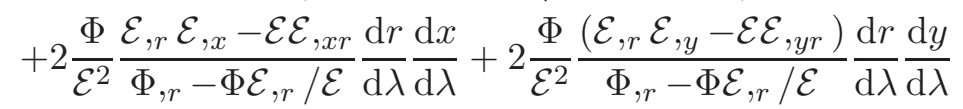

$$
\begin{aligned}
& -\frac{\Phi}{\mathcal{E}^{2}} \frac{1-k}{\Phi_{,_{r}}-\Phi \mathcal{E},_{r} / \mathcal{E}}\left[\left(\frac{\mathrm{d} x}{\mathrm{~d} \lambda}\right)^{2}+\left(\frac{\mathrm{d} y}{\mathrm{~d} \lambda}\right)^{2}\right]=0, \\
& \frac{\mathrm{d}^{2} x}{\mathrm{~d} \lambda^{2}}+2 \frac{\Phi, t}{\Phi} \frac{\mathrm{d} t}{\mathrm{~d} \lambda} \frac{\mathrm{d} x}{\mathrm{~d} \lambda}-\frac{1}{\Phi} \frac{\Phi,,_{r}-\Phi \mathcal{E},_{r} / \mathcal{E}}{1-k}\left(\mathcal{E}_{,_{r}} \mathcal{E}_{,_{x}}-\mathcal{E} \mathcal{E},_{x r}\right)\left(\frac{\mathrm{d} r}{\mathrm{~d} \lambda}\right)^{2} \\
& +\frac{2}{\Phi}\left(\Phi,,_{r}-\Phi \frac{\mathcal{E},,_{r}}{\mathcal{E}}\right) \frac{\mathrm{d} r}{\mathrm{~d} \lambda} \frac{\mathrm{d} x}{\mathrm{~d} \lambda}-\frac{\mathcal{E},,_{x}}{\mathcal{E}}\left(\frac{\mathrm{d} x}{\mathrm{~d} \lambda}\right)^{2}-2 \frac{\mathcal{E},{ }_{y}}{\mathcal{E}} \frac{\mathrm{d} x}{\mathrm{~d} \lambda} \frac{\mathrm{d} y}{\mathrm{~d} \lambda}+\frac{\mathcal{E},_{x}}{\mathcal{E}}\left(\frac{\mathrm{d} y}{\mathrm{~d} \lambda}\right)^{2}=0, \\
& \frac{\mathrm{d}^{2} y}{\mathrm{~d} \lambda^{2}}+2 \frac{\Phi, t}{\Phi} \frac{\mathrm{d} t}{\mathrm{~d} \lambda} \frac{\mathrm{d} y}{\mathrm{~d} \lambda}-\frac{1}{\Phi} \frac{\Phi,{ }_{r}-\Phi \mathcal{E},_{r} / \mathcal{E}}{1-k}\left(\mathcal{E}_{,_{r}} \mathcal{E}_{,_{y}}-\mathcal{E} \mathcal{E},{ }_{y r}\right)\left(\frac{\mathrm{d} r}{\mathrm{~d} \lambda}\right)^{2} \\
& +\frac{2}{\Phi}\left(\Phi, r-\Phi \frac{\mathcal{E},_{r}}{\mathcal{E}}\right) \frac{\mathrm{d} r}{\mathrm{~d} \lambda} \frac{\mathrm{d} y}{\mathrm{~d} \lambda}+\frac{\mathcal{E},_{y}}{\mathcal{E}}\left(\frac{\mathrm{d} x}{\mathrm{~d} \lambda}\right)^{2}-2 \frac{\mathcal{E},,_{x}}{\mathcal{E}} \frac{\mathrm{d} x}{\mathrm{~d} \lambda} \frac{\mathrm{d} y}{\mathrm{~d} \lambda}-\frac{\mathcal{E},_{y}}{\mathcal{E}}\left(\frac{\mathrm{d} y}{\mathrm{~d} \lambda}\right)^{2}=0,
\end{aligned}
$$

y la condición de geodésica nula es

$$
\left(\frac{\mathrm{d} t}{\mathrm{~d} \lambda}\right)^{2}-\frac{\left(\Phi_{, r}-\Phi \mathcal{E}_{, r} / \mathcal{E}\right)^{2}}{1-k}\left(\frac{\mathrm{d} r}{\mathrm{~d} \lambda}\right)^{2}-\frac{\Phi^{2}}{\mathcal{E}^{2}}\left[\left(\frac{\mathrm{d} x}{\mathrm{~d} \lambda}\right)^{2}+\left(\frac{\mathrm{d} y}{\mathrm{~d} \lambda}\right)^{2}\right]=0
$$

Las componentes del vector $n^{\alpha}$ pueden escribirse en términos de $k^{\alpha}$ como

$$
n^{i}=\frac{k^{i}}{k_{\alpha} u^{\alpha}}=-\frac{k^{i}}{k^{0}}
$$

Por otra parte, sabemos que el vector $n^{\alpha}$ indica la dirección de incidencia del fotón recibido en $\mathcal{O}$. Dado que nos interesa expresar esa dirección en términos de cantidades medibles (por ejemplo, los ángulos direccionales en coordenadas esféricas), y que, además, debe ser $n^{\alpha} n_{\alpha}=$ -1 , pasaremos a trabajar en coordenadas tales que el tensor métrico coincida con el de 
Minkowski en el punto de incidencia $\mathcal{O}{ }^{3}$ El cambio de coordenadas general presenta entonces dos partes: (i) la diagonalización de la métrica (en nuestro caso, todas las soluciones estudiadas están ya diagonalizadas), y (ii) la normalización, a través de un cambio de escala en cada una de las coordenadas, para absorber las funciones métricas evaluadas en el punto $\mathcal{O}$. Con tales consideraciones, tenemos que

$$
\begin{aligned}
\mathrm{d} s^{2} & =\mathrm{d} t^{2}-\frac{\left(\Phi^{\prime}-\Phi \mathcal{E}^{\prime} / \mathcal{E}\right)^{2}}{\epsilon-k(r)} \mathrm{d} r^{2}-\frac{\Phi^{2}}{\mathcal{E}^{2}}\left(\mathrm{~d} x^{2}+\mathrm{d} y^{2}\right) \\
& =\mathrm{d} t^{2}-\mathrm{d} \tilde{r}^{2}-\mathrm{d} \tilde{x}^{2}-\mathrm{d} \tilde{y}^{2} \\
& =\mathrm{d} t^{2}-\mathrm{d} \tilde{r}^{2}-\tilde{r}^{2}\left(\mathrm{~d} \theta^{2}+\operatorname{sen}^{2} \theta \mathrm{d} \phi^{2}\right)
\end{aligned}
$$

en donde la última igualdad involucra una transformación de las coordenadas $(\tilde{x}, \tilde{y})$ a las coordenadas angulares esféricas $(\theta, \phi)$ en el punto $\mathcal{O}$. Luego, el vector $n^{\alpha}$ queda expresado en términos de los ángulos de incidencia de los fotones en el punto $\mathcal{O}$, de la forma

$$
n^{\alpha}=\left[0,-\operatorname{sen} \theta_{o} \cos \phi_{o},-\operatorname{sen} \theta_{o} \operatorname{sen} \phi_{o},-\cos \theta_{o}\right] .
$$

La componentes del vector de onda y su derivada deben ser también expresadas en coordenadas esféricas. Para ello, se realiza una transformación del tipo

$$
\begin{array}{rlrl}
k^{i} & =M k^{i^{\prime}} & \leftrightarrow & k^{i^{\prime}}=M^{-1} k^{i}, \\
\dot{k}^{i}=M \dot{k}^{i^{\prime}} & \leftrightarrow \quad \dot{k}^{i^{\prime}}=M^{-1} \dot{k}^{i},
\end{array}
$$

en donde los índices $i$ e $i^{\prime}$ indican las componentes de los vectores expresadas, respectivamente, en coordenadas esféricas y coordenadas $\{t, \tilde{r}, \tilde{x}, \tilde{y}\}$, y $\left(M, M^{-1}\right)$ son las matrices de transformación entre ambos sistemas coordenados.

El modelo quasi-esférico de Székeres describe un fluido sin rotación y con presión nula, y las cantidades cinemáticas están dadas por

$$
\begin{aligned}
a^{\alpha} & =\omega^{\alpha \beta}=0 \\
\Theta & =\frac{\dot{\Phi}^{\prime}+2 \dot{\Phi} \Phi^{\prime} / \Phi-3 \dot{\Phi} \mathcal{E}^{\prime} / \mathcal{E}}{\Phi^{\prime}-\Phi \mathcal{E}^{\prime} / \mathcal{E}}, \\
\sigma_{\beta}^{\alpha} & =\frac{1}{3}\left(\frac{\dot{\Phi}^{\prime}-\dot{\Phi} \Phi^{\prime} / \Phi}{\Phi^{\prime}-\Phi \mathcal{E}^{\prime} / \mathcal{E}}\right) \operatorname{diag}(0,2,-1,-1), \\
E_{\beta}^{\alpha} & =C_{\gamma \beta \delta}^{\alpha} u^{\gamma} u^{\delta}=\frac{M\left(3 \Phi^{\prime}-\Phi M^{\prime} / M\right)}{3 \Phi^{3}\left(\Phi^{\prime}-\Phi \mathcal{E}^{\prime} / \mathcal{E}\right)} \operatorname{diag}(0,2,-1,-1) .
\end{aligned}
$$

Para trabajar con la fórmula (3.12), analicemos algunos términos en forma individual. Aquellos que involucran al shear, resultan

$$
\begin{aligned}
n_{\alpha} n_{\beta} \sigma^{\alpha \beta} & =\operatorname{sen}^{2} \theta_{o} \cos ^{2} \phi_{o} \Sigma_{1}+\operatorname{sen}^{2} \theta_{o} \operatorname{sen}^{2} \phi_{o} \Sigma_{2}+\cos ^{2} \theta_{o} \Sigma_{3}, \\
n_{\alpha} n_{\beta} \sigma_{\gamma}{ }_{\gamma}^{\alpha} \sigma^{\beta>\gamma} & =-\left(\operatorname{sen}^{2} \theta_{o} \cos ^{2} \phi_{o} \Sigma_{1}^{2}+\operatorname{sen}^{2} \theta_{o} \operatorname{sen}^{2} \phi_{o} \Sigma_{2}^{2}+\cos ^{2} \theta_{o} \Sigma_{3}^{2}\right), \\
\sigma_{\alpha \beta} n^{\alpha} k^{\beta} & =\left(\operatorname{sen}^{2} \theta_{o} \cos ^{2} \phi_{o} \Sigma_{1} k^{1}+\operatorname{sen}^{2} \theta_{o} \operatorname{sen}^{2} \phi_{o} \Sigma_{2} k^{2}+\cos ^{2} \theta_{o} \Sigma_{3} k^{3}\right), \\
\sigma_{\alpha \beta} n^{\alpha} \dot{k}^{\beta} & =\left(\operatorname{sen}^{2} \theta_{o} \cos ^{2} \phi_{o} \Sigma_{1} \dot{k}^{1}+\operatorname{sen}^{2} \theta_{o} \operatorname{sen}^{2} \phi_{o} \Sigma_{2} \dot{k}^{2}+\cos ^{2} \theta_{o} \Sigma_{3} \dot{k}^{3}\right),
\end{aligned}
$$

\footnotetext{
${ }^{3}$ La existencia de estas coordenadas está garantizada por el Principio de Equivalencia.
} 
en donde todas las cantidades están expresadas en el sistema de coordenadas esféricas en el punto $\mathcal{O}$, y hemos considerado

$$
\sigma_{\alpha \beta}=\frac{1}{3} \Sigma \operatorname{diag}\left(0,2 g_{r r},-g_{\theta \theta},-g_{\phi \phi}\right) \equiv \operatorname{diag}\left(0, \Sigma_{1}, \Sigma_{2}, \Sigma_{3}\right),
$$

con

$$
\Sigma_{1}+\Sigma_{2}+\Sigma_{3}=0 .
$$

Además, dado que la parte eléctrica del tensor de Weyl es en este caso no nula, tenemos que

$$
n_{\alpha} n_{\beta} E^{\alpha \beta}=n^{\alpha} n^{\beta} E_{\alpha \beta}=\left(n^{1}\right)^{2} E_{1}+\left(n^{2}\right)^{2} E_{2}+\left(n^{3}\right)^{2} E_{3},
$$

con

$$
E_{\alpha \beta}=g_{\beta}^{\alpha} E_{\beta}^{\alpha}=E \operatorname{diag}\left(0,2 g_{r r},-g_{\theta \theta},-g_{\phi \phi}\right) \equiv \operatorname{diag}\left(0, E_{1}, E_{2}, E_{3}\right) .
$$

Con las consideraciones anteriores, la aproximación covariante para fuentes cercanas dada por la ecuación (3.12) resulta

$$
\begin{aligned}
& \frac{\delta z}{\delta t_{o}}=\left\{\frac{2}{3} \Theta\left[\left(n^{1}\right)^{2} \Sigma_{1}+\left(n^{2}\right)^{2} \Sigma_{2}+\left(n^{3}\right)^{2} \Sigma_{3}\right]-\left[\left(n^{1}\right)^{2} \Sigma_{1}^{2}+\left(n^{2}\right)^{2} \Sigma_{2}^{2}+\left(n^{3}\right)^{2} \Sigma_{3}^{2}\right]\right. \\
& +\left[\left(n^{1}\right)^{2} E_{1}^{2}+\left(n^{2}\right)^{2} E_{2}^{2}+\left(n^{3}\right)^{2} E_{3}^{2}\right]+\frac{2}{k^{0}}\left(\Sigma_{1} n^{1} \dot{k}^{1}+\Sigma_{2} n^{2} \dot{k}^{2}+\Sigma_{3} n^{3} \dot{k}^{3}\right) \\
& -2 \frac{\dot{k}^{0}}{\left(k^{0}\right)^{2}}\left(\Sigma_{1} n^{1} k^{1}+\Sigma_{2} n^{2} k^{2}+\Sigma_{3} n^{3} k^{3}\right)-\left[\Sigma_{1}\left(n^{1}\right)^{2}+\Sigma_{2}\left(n^{2}\right)^{2}+\Sigma_{3}\left(n^{3}\right)^{2}\right]^{2} \\
& \left.-\frac{2}{3} \sigma^{2}-\frac{1}{6} \rho_{m}\right\}_{o} \frac{z}{\left\{\frac{1}{3} \Theta-\left[\left(n^{1}\right)^{2} \Sigma_{1}+\left(n^{2}\right)^{2} \Sigma_{2}+\left(n^{3}\right)^{2} \Sigma_{3}\right]\right\}_{o}},
\end{aligned}
$$

y ordenando los términos en forma conveniente, obtenemos

$$
\begin{array}{r}
\frac{\delta z}{\delta t_{o}}=\left\{\left(n^{1}\right)^{2}\left[\frac{2}{3} \Theta \Sigma_{1}-\Sigma_{1}^{2}+E_{1}+2 \frac{k^{0}}{k^{0}} \Sigma_{1}\right]+\left(n^{2}\right)^{2}\left[\frac{2}{3} \Theta \Sigma_{2}-\Sigma_{2}^{2}+E_{2}+2 \frac{\dot{k}^{0}}{k^{0}} \Sigma_{2}\right]\right. \\
+\left(n^{3}\right)^{2}\left[\frac{2}{3} \Theta \Sigma_{3}-\Sigma_{3}^{2}+E_{3}+2 \frac{\dot{k}^{0}}{k^{0}} \Sigma_{3}\right]-\left[\Sigma_{1}\left(n^{1}\right)^{2}+\Sigma_{2}\left(n^{2}\right)^{2}+\Sigma_{3}\left(n^{3}\right)^{2}\right]^{2} \\
+\frac{2}{k^{0}}\left(\Sigma_{1} n^{1} \dot{k}^{1}+\Sigma_{2} n^{2} \dot{k}^{2}+\Sigma_{3} n^{3} \dot{k}^{3}\right) \\
\left.-\frac{2}{3} \sigma^{2}-\frac{1}{6} \rho_{m}\right\}_{o} \frac{z}{\left\{\frac{1}{3} \Theta-\left[\left(n^{1}\right)^{2} \Sigma_{1}+\left(n^{2}\right)^{2} \Sigma_{2}+\left(n^{3}\right)^{2} \Sigma_{3}\right]\right\}_{o}}
\end{array}
$$

La ecuación (3.46) corresponde a la aproximación covariante del redshift drift de fuentes cercanas en el modelo cuasiesférico de Székeres, en donde todas las cantidades involucradas están expresadas en coordenadas esféricas en el punto $\mathcal{O}$.

Para analizar un ejemplo simple, consideremos un modelo cuasiesférico tal que $\sigma^{\alpha \beta} \simeq 0$. En este caso, la aproximación covariante se reduce a

$$
\frac{\delta z}{\delta t_{o}}=\left\{\left(n^{1}\right)^{2} E_{1}+\left(n^{2}\right)^{2} E_{2}+\left(n^{3}\right)^{2}+E_{3}-\frac{1}{6} \rho_{m}\right\}_{o} \frac{z}{\Theta_{o} / 3},
$$


y la contribución anisótropa es introducida sólo a través de la parte eléctrica del tensor de Weyl (que en el caso de FLRW resulta idénticamente nula). Vemos entonces que la expresión (3.47) permitiría entonces diferenciar a los modelos cuasiesféricos de Székeres, con valores muy pequeños (despreciables) del shear, del modelo $\Lambda$ CDM.

\section{Modelos de Bianchi tipo I:}

Estudiaremos, por último, el redshift drift en el caso de los modelos de Bianchi tipo I (ver apéndice $\mathrm{C}$ ), en donde las contribuciones anisótropas se pueden identificar explícitamente. La simetría axial presente en estos modelos hace que puedan ser comparados directamente con un modelo de LTB con el punto de referencia $\mathcal{O}$ ubicado fuera del centro de simetría [185].

El elemento de línea que describe a la métrica, en coordenadas comóviles al fluido cosmológico de materia con velocidad $u^{a}=\delta_{0}^{a}$, viene dado por [186]

$$
\mathrm{d} s^{2}=-\mathrm{d} t^{2}+a_{1}^{2}(t) \mathrm{d} x^{2}+a_{2}^{2}(t) \mathrm{d} y^{2}+a_{3}^{2}(t) \mathrm{d} z^{2} .
$$

El vector de onda, $k^{\alpha} \equiv \mathrm{d} x^{\alpha} / \mathrm{d} \lambda$, se obtiene integrando la ecuación de la geodésica para cada componente [185], a saber,

$$
\begin{aligned}
\frac{\mathrm{d} k^{0}}{\mathrm{~d} \lambda} & =-\sum_{i} H_{i}\left(a_{i}^{2} k^{i}\right)^{2}, \\
\frac{\mathrm{d} k^{i}}{\mathrm{~d} \lambda} & =-2 H_{i} \frac{\mathrm{d} t}{\mathrm{~d} \lambda} k^{i}, \quad i=1,2,3,
\end{aligned}
$$

con $H_{i}(t) \equiv \dot{a}_{i}(t) / a_{i}(t), i=1,2,3$. Luego, resulta

$$
\mathrm{d} k^{i}=-2 H_{i} k^{i} \mathrm{~d} t \rightarrow k^{i}(t)=k_{o}^{i} \frac{1}{a_{i}(t)^{2}},
$$

y, teniendo en cuenta la condición de geodésica nula, $k^{\alpha} k_{\alpha}=0$, obtenemos

$$
\begin{aligned}
\left(\frac{\mathrm{d} t}{\mathrm{~d} \lambda}\right)^{2} & =\sum_{i}\left(a_{i} k^{i}\right)^{2}=\sum_{i}\left[\frac{k_{o}^{i}}{\left[a_{i}(t(\lambda))\right]^{2}}\right] \\
\rightarrow k^{\alpha} & =\left( \pm \sqrt{\sum_{i}\left[\frac{k_{o}^{i}}{a_{i}(t)}\right]^{2}}, \frac{k_{o}^{i}}{a_{i}^{2}(t)}\right), \quad i=1,2,3,
\end{aligned}
$$

en donde $k_{o}^{i}=k^{i}\left(t_{o}\right)$.

Como en el caso del modelo de Székeres, conviene trabajar en coordenadas tales que el métrico coincida con el de Minkowski en el punto de incidencia $\mathcal{O}$. El vector normal queda entonces expresado como

$$
n^{\alpha}=\left[0,-\operatorname{sen} \theta_{o} \cos \phi_{o},-\operatorname{sen} \theta_{o} \operatorname{sen} \phi_{o},-\cos \theta_{o}\right] .
$$

Teniendo en cuenta que las cantidades cinemáticas se escriben

$$
\begin{aligned}
\Theta & =H_{1}+H_{2}+H_{3}, \\
\sigma^{\alpha \beta} & =\operatorname{diag}\left[0, \Sigma_{1}, \Sigma_{2}, \Sigma_{3}\right], \quad \operatorname{con} \Sigma_{1}+\Sigma_{2}+\Sigma_{3}=0,
\end{aligned}
$$


tenemos

$$
\begin{aligned}
n_{\alpha} n_{\beta} \sigma^{\alpha \beta} & =\operatorname{sen}^{2} \theta_{o} \cos ^{2} \phi_{o} \Sigma_{1}+\operatorname{sen}^{2} \theta_{o} \operatorname{sen}^{2} \phi_{o} \Sigma_{2}+\cos ^{2} \theta_{o} \Sigma_{3}, \\
n_{\alpha} n_{\beta} \sigma_{\gamma}{ }^{<\alpha} \sigma^{\beta>\gamma} & =-\left(\operatorname{sen}^{2} \theta_{o} \cos ^{2} \phi_{o} \Sigma_{1}^{2}+\operatorname{sen}^{2} \theta_{o} \operatorname{sen}^{2} \phi_{o} \Sigma_{2}^{2}+\cos ^{2} \theta_{o} \Sigma_{3}^{2}\right), \\
\sigma_{\alpha \beta} n^{\alpha} k^{\beta} & =-k^{0}\left(\operatorname{sen}^{2} \theta_{o} \cos ^{2} \phi_{o} \Sigma_{1}+\operatorname{sen}^{2} \theta_{o} \operatorname{sen}^{2} \phi_{o} \Sigma_{2}+\cos ^{2} \theta_{o} \Sigma_{3}\right), \\
\sigma_{\alpha \beta} n^{\alpha} \dot{k}^{\beta} & =2\left(k^{0}\right)^{2}\left(\operatorname{sen}^{2} \theta_{o} \cos ^{2} \phi_{o} \Sigma_{1} H_{1}+\operatorname{sen}^{2} \theta_{o} \operatorname{sen}^{2} \phi_{o} \Sigma_{2} H_{2}+\cos ^{2} \theta_{o} \Sigma_{3} H_{3}\right),
\end{aligned}
$$

en donde hemos tenido en cuenta que $\left(k_{\alpha} u^{\alpha}\right)=k^{0}$ y $k^{i}=-n^{i} k^{0}$. Notemos que las cantidades anteriores se calculan formalmente igual que en el caso de Székeres. Con todo lo anterior, la fórmula (3.12) resulta

$$
\begin{aligned}
\frac{\delta z}{\delta t_{o}}= & \left\{\frac{2}{3} \Theta \sum_{i}\left(\Sigma_{i}\left(n^{i}\right)^{2}\right)-\sum_{i}\left(\Sigma_{i}^{2}\left(n^{i}\right)^{2}\right)-\left[\sum_{i}\left(\Sigma_{i}\left(n^{i}\right)^{2}\right)\right]^{2}+4 k^{0} \sum_{i}\left(\Sigma_{i} H_{i}\left(n^{i}\right)^{2}\right)\right. \\
& \left.-2 \frac{\dot{k}^{0}}{k^{0}} \sum_{i}\left(\Sigma_{i}\left(n^{i}\right)^{2}\right)-\frac{2}{3} \sigma^{2}-\frac{1}{6}(\rho+3 p)\right\}_{o} \frac{z}{\left\{\sum_{i} H_{i}\left(n^{i}\right)^{2}-\sum_{i} \Sigma_{i}\left(n^{i}\right)^{2}\right\}_{o}}
\end{aligned}
$$

Consideraremos ahora los modelos de Bianchi tipo I, es decir, aquellos tales que $H_{a} \equiv$ $H_{1}=H_{2}, H_{b} \equiv H_{3}, \Sigma_{a} \equiv \Sigma_{1}=\Sigma_{2}, \Sigma_{b} \equiv \Sigma_{3}$. Como mencionamos anteriormente, estos modelos anisótropos pueden compararse directamente con aquellos modelos de LTB con puntos de referencia fuera del centro de simetría. ${ }^{4}$ La aproximación covariante del redshift drift para fuentes cercanas puede escribirse en este caso como

$$
\begin{aligned}
\left(\frac{\delta z}{\delta t}\right)_{o}=\left\{\operatorname{sen}^{2} \theta\left[\frac{2}{3}\left(2 H_{a}+H_{b}\right) \Sigma_{a}-\Sigma_{a}^{2}+4 \Sigma_{a} H_{a}\right]\right. \\
+\cos ^{2} \theta\left[\frac{2}{3}\left(2 H_{a}+H_{b}\right) \Sigma_{b}-\Sigma_{b}^{2}+4 \Sigma_{b} H_{b}\right]-\left[\Sigma_{a} \operatorname{sen}^{2} \theta+\Sigma_{b} \cos ^{2} \theta\right]^{2} \\
+4\left(H_{a} \operatorname{sen}^{2} \theta+H_{b} \cos ^{2} \theta\right)\left[\Sigma_{a} \operatorname{sen}^{2} \theta+\Sigma_{b} \cos ^{2} \theta\right]^{2} \\
\\
\left.\quad-\frac{2}{3} \sigma^{2}-\frac{1}{6}(\rho+3 p)\right\}_{o} \frac{z}{\left(H_{a}-\Sigma_{a}\right)_{o} \operatorname{sen}^{2} \theta_{o}+\left(H_{b}-\Sigma_{b}\right)_{o} \cos ^{2} \theta_{o}} \cdot(3.59
\end{aligned}
$$

La dependencia angular de la ecuación (3.59) en relación a la dirección de incidencia de los fotones hace evidente la anisotropía del modelo, que depende del shear y el escalar de expansión. Para el caso particular en que $H_{a}=H_{b}$ y $\Sigma_{a}=\Sigma_{b}=0$, la geometría resulta isótropa y se recupera la expresión (3.17) de los modelos de FLRW.

\subsubsection{Discusión}

Hemos presentado en esta sección una formula aproximada para calcular el redshift drift de fuentes cercanas, a partir de la descomposición $1+3$. El formalismo en el que trabajamos es

\footnotetext{
${ }^{4}$ En esta dirección, un trabajo interesante que compara las predicciones de la paralaje cósmica para ambos modelos fue presentado en [185].
} 
covariante, y lo por tanto válido para cualquier sistema de coordenadas, dentro del marco de la Relatividad General. Además, el formalismo puede ser aplicado a observaciones realizadas en cualquier punto arbitrario $\mathcal{O}$.

A modo de ejemplo, calculamos el redshift drift para diferentes geometrías de interés cosmológico. Del análisis de nuestros resultados, podemos destacar diferentes aplicaciones para la aproximación covariante que hemos desarrollado. A saber:

(I) La posibilidad de identificar un signo definido en el redshift drift de fuentes cercanas, como principal herramienta para distinguir entre modelos cosmológicos.

Un claro ejemplo de esta aplicación es el caso del modelo $\Lambda$ CDM y de los modelos de void de LTB. Comparando las ecuaciones (3.17) y (3.21) encontramos que, mientras que en el modelo $\Lambda \mathrm{CDM}$ el redshift drift es definido positivo para fuentes cercanas, en los modelos de LTB resulta siempre negativo. Las perspectivas observacionales que discutiremos más adelante (sección 3.4) son, en este sentido, prometedoras para la tarea de poder distinguir estos modelos en un futuro próximo.

Cabe destacar, asimismo, que los resultados que obtuvimos para el caso de las soluciones de FLRW y de LTB coinciden con aquellos presentados previamente por otros autores [88], con la ventaja de no necesitar de la integración de las ecuaciones geodésicas que determinan la trayectoria de los fotones. ${ }^{5}$

(II) El estudio de posibles anisotropías, descriptas por soluciones con menos simetrías, como la de Székeres o la de Bianchi.

La fórmula covariante aplicada a modelos con contribuciones anisótropas, como los cuasiesféricos de Székeres o los de Bianchi tipo I, evidencian la dependencia angular del redshift drift con la dirección de incidencia de los fotones. Los modelos de Bianchi tipo I, por su parte, pueden ser directamente comparados con aquellos de LTB con puntos de medición fuera del centro de simetría. En este sentido, es interesante profundizar su estudio como un primer paso para el análisis de casos más complejos.

La paralaje cósmica es otro observable a tiempo real que puede servir para cuantificar la expansión anisótropa [88, 159]. El cálculo de dicha cantidad para el caso de los modelos de Bianchi tipo I, y su comparación con modelo de LTB para fotones que inciden fuera del centro de simetría, fue presentado en [159, 185]. En este sentido, las futuras mediciones del redshfit drift y de la paralaje cósmica son complementarias, incluso para poner cotas sobre la distancia del punto $\mathcal{O}$ respecto al centro de simetría (para el caso de modelos esféricos con observadores no centrales).

(III) La posibilidad de estudiar el redshift drift en modelos con rotación no nula, a través del parámetro $\omega^{\alpha}$.

En general, dado que cualquier vestigio de rotación cósmica debería haber sido diluido durante el proceso de inflación [187], la rotación global no es tenida en cuenta en los modelos cosmológicos. Sin embargo, ciertas regiones espaciales extendidas, como voids a grandes escalas o cúmulos de galaxias, pueden poseer un momento angular no nu-

\footnotetext{
${ }^{5}$ La aproximación del redshift drift presentada en [174], para el caso de modelos de void de LTB y fuentes cercanas, tampoco fue obtenida a partir de la integración de las geodésicas. Sin embargo, el cálculo involucrado en dicho trabajo es más complejo y menos general que el presentado aquí.
} 
lo [188]. ${ }^{6}$ En esta dirección, un trabajo interesante sobre la posibilidad de distinguir modelos anisótropos con rotación fue presentado en [160].

Dado que la aproximación covariante que presentamos depende explícitamente del vector de vórtice $\omega^{\alpha}$, el redshift drift puede ser también utilizado para estudiar este tipo de modelos con rotación no nula.

Por último, queremos destacar que la dependencia angular explícita de la expresión (3.12) puede servir para inferir posibles vínculos entre la magnitud de $\left(\delta z / \delta t_{0}\right)$ con el ángulo de incidencia de los fotones. En este sentido, la aproximación covariante que hemos presentado podría ser utilizada también para optimizar los proyectos observacionales abocados a la medición del redshift drift (ver sección 3.4).

\subsection{Aproximación cosmográfica del redshift drift $(z \ll 1)$}

Motivados por estudiar cuánto de la cosmología moderna puede conocerse sólo a partir de principios geométricos y observaciones directas (es decir, desde un punto de vista cosmográfico ${ }^{7}$ ), presentaremos a continuación la estimación del redshift drift de fuentes cercanas en modelos homogéneos e isótropos a partir de un tratamiento cosmográfico.

Consideremos el redshift de un fotón emitido por una dada fuente y recibido en un punto $\mathcal{O}$ al instante $t_{o}$, definido como

$$
z \equiv \frac{\left(u^{\alpha} k_{\alpha}\right)_{e}}{\left(u^{\alpha} k_{\alpha}\right)_{o}}-1
$$

en donde $u^{\alpha}$ es la velocidad del sistema de referencia del punto $\mathcal{O}$ y $k^{\alpha}$ es el vector de onda de un fotón incidente. Para el caso de un modelo cosmológico basado en la métrica homogénea e isótropa de FLRW, resulta

$$
z\left(t_{o}\right)=\frac{a\left(t_{o}\right)}{a\left(t_{e}\right)}-1
$$

en donde $a(t)$ es el factor de escala de la métrica y $t_{e}$ es el instante en el que la luz fue emitida. Luego de un intervalo de tiempo $\delta t_{o}$ ( $\delta t_{e}$ para la fuente) resulta

$$
z\left(t_{o}+\delta t_{o}\right)=\frac{a\left(t_{o}+\delta t_{o}\right)}{a\left(t_{e}+\delta t_{e}\right)}-1 .
$$

Durante dicho intervalo, la variación temporal del redshift de la fuente, a primer orden en $\delta t_{o}$ y $\delta t_{e}$, resulta [164]

$$
\delta z=\frac{a\left(t_{o}+\delta t_{o}\right)}{a\left(t_{e}+\delta t_{e}\right)}-\frac{a\left(t_{o}\right)}{a\left(t_{e}\right)} .
$$

La relación entre ambos instantes de tiempo viene dada por [59]

$$
\int_{t_{e}}^{t_{o}} \frac{\mathrm{d} t}{a(t)}=\int_{t_{e}+\delta t_{e}}^{t_{o}+\delta t_{o}} \frac{\mathrm{d} t}{a(t)}
$$

\footnotetext{
${ }^{6}$ Modelos con rotación a gran escala y modelos con rotación global han sido investigados en [189, 190], y referencias allí citadas.

${ }^{7}$ Dentro de la cosmología, llamamos cosmografía al estudio del universo basado sólo en el Principio Cosmológico y en aquellas partes de la Teoría de la Relatividad General que se desprenden del Principio de Equivalencia. Para una discusión más detallada, ver [59] (capítulo 14) y [191].
} 
de donde resulta $\delta t_{e}=\left[a\left(t_{e}\right) / a\left(t_{o}\right)\right] \delta t_{o}$. Luego, utilizando la expansión en series de Taylor del factor de escala para intervalos temporales pequeños $(\delta t / t \ll 1)$, y expresada a primer orden como $a(t+\delta t) \simeq a(t)+\dot{a}(t) \delta t$, obtenemos [164]

$$
\frac{\delta z}{\delta t_{o}} \simeq \frac{\dot{a}\left(t_{o}\right)-\dot{a}\left(t_{e}\right)}{a\left(t_{e}\right)}=\frac{\dot{z}\left(t_{e}\right)}{1+z}-(1+z) \dot{z}\left(t_{o}\right),
$$

en donde el símbolo ' indica derivadas temporales, y tuvimos en cuenta la relación $a(t)=$ $(1+z)^{-1}$. La ecuación (3.65) es la expresión general del redshift drift de la fuente en un modelo isótropo y homogéneo para intervalos de tiempo pequeños. Notemos que la variación temporal del redshift cosmológico resulta proporcional a la tasa de expansión durante la evolución del universo (esto es, a su aceleración o desaceleración) y, en consecuencia, será una cantidad nula si durante algún intervalo temporal el universo mantiene su expansión constante.

Una manera conveniente de estudiar el redshift drift es expresar $\left(\delta z / \delta t_{0}\right)$ términos del parámetro de expansión de Hubble, $H(t) \equiv \dot{a}(t) / a(t)$. La variación temporal del redshift cosmológico puede escribirse entonces como

$$
\frac{\delta z}{\delta t_{o}} \simeq a\left(\dot{t}_{o}\right)\left[\frac{1}{a\left(t_{e}\right)}-\frac{1}{\dot{a}\left(t_{o}\right)} \frac{\dot{a}\left(t_{e}\right)}{a\left(t_{e}\right)}\right]=H\left(t_{o}\right)\left[1+z-\frac{H\left(t_{e}\right)}{H\left(t_{o}\right)}\right]
$$

en donde hemos considerado $a\left(t_{o}\right)=1$ y la relación $a\left(t_{e}\right)=(1+z)^{-1}$. Denotando $H\left(t_{o}\right)=H_{o}$ y $H\left(t_{e}\right)=H(t)$, resulta

$$
\frac{\delta z}{\delta t_{o}}=H_{o}\left[1+z-\frac{H(t)}{H_{o}}\right]
$$

La ecuación (3.67) es la expresión general del redshift drift para fuentes cercanas en modelos homogéneos e isótropos. Como mencionamos anteriormente, el objetivo del trabajo presentado en esta sección es justamente abordar el análisis del redshift drift desde un punto de vista cosmográfico. Notemos entonces que la única hipótesis que hemos considerado hasta aquí fue la de trabajar con la métrica de FLRW, por lo que este resultado es independiente de la dinámica del modelo cosmológico que estemos estudiando, cuya información está contenida únicamente en la función $H(t)$.

Nos abocaremos ahora a estudiar las propiedades que se desprenden de la simetría de la métrica y de los datos observacionales actuales, sin especificar una dinámica particular. Si bien no es posible reconstruir la evolución de $a(t)$ a lo largo de toda la historia del universo a partir de observaciones astronómicas, podemos estimar el valor actual del factor de escala y de sus derivadas a través de los parámetros cinemáticos cosmológicos [191]. Adoptando la terminología comúnmente usada en cosmografía, definimos los llamados parámetros cinemáticos en función del factor de escala y sus derivadas de la siguiente manera:

$$
\begin{aligned}
\text { parámetro de Hubble } & \rightarrow H(t) \equiv+\frac{1}{a(t)} \frac{\mathrm{d} a}{\mathrm{~d} t}, \\
\text { parámetro de desaceleración } & \rightarrow q(t) \equiv-\frac{1}{a(t)} \frac{\mathrm{d}^{2} a}{\mathrm{~d} t^{2}}\left[\frac{1}{a(t)} \frac{\mathrm{d} a}{\mathrm{~d} t}\right]^{-2}, \\
\text { parámetro jerk } & \rightarrow j(t) \equiv+\frac{1}{a(t)} \frac{\mathrm{d}^{3} a}{\mathrm{~d} t^{3}}\left[\frac{1}{a(t)} \frac{\mathrm{d} a}{\mathrm{~d} t}\right]^{-3}, \\
\text { parámetro snap } & \rightarrow s(t) \equiv+\frac{1}{a(t)} \frac{\mathrm{d}^{4} a}{\mathrm{~d} t^{4}}\left[\frac{1}{a(t)} \frac{\mathrm{d} a}{\mathrm{~d} t}\right]^{-4},
\end{aligned}
$$


en donde las unidades de $H$ suelen estar expresadas en $\mathrm{km} / \mathrm{s} / \mathrm{Mpc}$, y los últimos tres parámetros resultan adimensionales. Con las definiciones anteriores, podemos escribir el desarrollo en series de Taylor del factor de escala en torno al instante $t_{o}$ en el que los fotones son medidos como [191]

$$
\begin{aligned}
a(t)=a_{o}\left[1+H_{o}(t\right. & \left.-t_{o}\right)-\frac{1}{2} q_{o} H_{o}^{2}\left(t-t_{o}\right)^{2} \\
& \left.+\frac{1}{6} j_{o} H_{o}^{3}\left(t-t_{o}\right)^{3}+\frac{1}{24} s_{o} H_{o}^{4}\left(t-t_{o}\right)^{4}\right]+\mathcal{O}\left(\left[t-t_{o}\right]^{5}\right) .
\end{aligned}
$$

Si bien este desarrollo es exacto, en la práctica no puede ser utilizado directamente para el cálculo del redshift drift, pues desconocemos el instante de tiempo $t=t_{e}$ en el que la luz fue emitida desde la fuente. Es necesario entonces reescribir $t_{e}$ en términos de cantidades físicas conocidas. Para ello, tenemos en cuenta que la distancia física que recorre un fotón emitido en el instante $t_{e} \mathrm{y}$ medido en un tiempo $t_{o}$ es

$$
D=c \int \mathrm{d} t=c\left(t_{o}-t_{e}\right)
$$

Luego, $t_{e}=t_{o}-D / c$, haciendo uso de la definición (3.61), resulta [191]

$$
\begin{aligned}
1+z & =\frac{a\left(t_{o}\right)}{a\left(t_{o}-D / c\right)} \\
& =1+\frac{H_{o} D}{c}+\frac{2+q_{o}}{2} \frac{H_{o}^{2} D^{2}}{c^{2}}+\frac{6\left(1+q_{o}\right)+j_{o}}{6} \frac{H_{o}^{3} D^{3}}{c^{3}}+\mathcal{O}\left(\left[\frac{H_{o} D}{c}\right]^{4}\right), \\
\Rightarrow z(D) & =\frac{H_{o} D}{c}+\frac{2+q_{o}}{2} \frac{H_{o}^{2} D^{2}}{c^{2}}+\frac{6\left(1+q_{o}\right)+j_{o}}{6} \frac{H_{o}^{3} D^{3}}{c^{3}}+\mathcal{O}\left(\left[\frac{H_{o} D}{c}\right]^{4}\right),
\end{aligned}
$$

en donde hemos trabajado con el desarrollo en series de Taylor hasta cuarto orden en la variable adimensional $\left(D H_{o} / c\right)$. Invirtiendo la serie de potencias dada por la ecuación (3.74) (esto es, $z(D) \rightarrow D(z))$, obtenemos [191]

$$
\begin{aligned}
& D(z)=\frac{c z}{H_{o}}\left[1-\left(1+\frac{q_{o}}{2}\right) z+\left(1+q_{o}+\frac{q_{o}^{2}}{2}-\frac{j_{o}}{6}\right) z^{2}\right. \\
&\left.-\left(1+\frac{3}{2} q_{o}\left(1+q_{o}\right)+\frac{5}{8} q_{o}^{3}-\frac{1}{2} j_{o}-\frac{5}{12} q_{o} j_{o}-\frac{s_{o}}{24}\right) z^{3}\right]+\mathcal{O}\left(z^{4}\right) .
\end{aligned}
$$

Desarrollando entonces la expresión (3.61) en serie de potencias de la cantidad $\left(H_{o} D / c\right), y$ teniendo en cuenta la ecuación (3.75), la variación temporal del redshift cosmológico puede aproximarse a tercer orden en $z$ como

$$
\frac{\delta z}{\delta t_{o}} \simeq-H_{o} q_{o} z+\frac{1}{2} H_{o}\left(q_{o}^{2}-j_{o}\right) z^{2}+\frac{1}{2} H_{o}\left[\frac{1}{3}\left(s_{o}+4 q_{o} j_{o}\right)+j_{o}-q_{o}^{2}-q_{o}^{3}\right] z^{3} .
$$

La ezpresión (3.76), válida para fuentes cercanas $(z \ll 1)$, indica el redshift drift de una fuente en función de su redshift cosmológico para modelos construidos con la métrica de FLRW, sin especificar ninguna dinámica particular para la evolución del factor de escala. 
Todos aquellos modelos cosmológicos que estén basados en la métrica de FLRW deben satisfacer las restricciones observacionales que impone la relación cinemática del redshift drift para $z$ bajos. Por otra parte, la aproximación (3.76) puede utilizarse para comparar el redshift drift cinemático con aquel estimado a partir de la evolución exacta del factor de escala de un dado modelo. Dicha comparación puede ser utilizada, por ejemplo, para imponer restricciones sobre la dinámica de modelos cosmológicos homogéneos e isótropos, incluso en el marco de otras teorías de gravedad, como las teorías $f(R)$.

En esta dirección, desarrollamos un método basado en la comparación de cantidades observables estimadas a partir de sus tratamientos cosmográfico y dinámico. Dicha comparación es realizada utilizando desarrollos en series de potencias del redshift, y el método puede ser aplicado a cualquier observable cosmológico que pueda ser escrito en términos del parámetro de Hubble, $H(z)$. Una aplicación interesante de este método de comparación tiene lugar en aquellos modelos cosmológicos desarrollados en el marco de las teorías de gravedad modificada $f(R)$ y basados también en la métrica de FLRW. En particular, el tratamiento cinemático del reshift drift puede ser utilizado en estos casos para obtener cotas sobre los parámetros libres que presentan estos modelos. El desarrollo del método mencionado, y una aplicación directa al estudio de dos modelos cosmológicos en el marco de teorías $f(R)$, están detallados en el apéndice E. Este trabajo fue publicado en [192] y representa un complemento del trabajo de investigación original que forma parte de esta tesis.

Para finalizar, a modo de ejemplo, comparemos la aproximación cosmográfica (3.76) con el redshift drift calculado a partir de la dinámica del modelo $\Lambda$ CDM. En este caso, las ecuaciones de Friedmann-Lemaître pueden ser utilizadas en la ecuación (3.67) para relacionar la variación temporal del redshift cosmológico con el contenido de materia del universo [164]. La ecuación que determina la evolución dinámica del factor de escala, para el caso de curvatura nula, viene dada por

$$
\left[\frac{\dot{a}(t)}{a(t)}\right]^{2}=H_{0}^{2}\left[\Omega_{m, 0} a(t)^{-3}+\Omega_{r, 0} a(t)^{-4} \Omega_{\Lambda, 0}\right],
$$

en donde $\Omega_{m, 0}, \Omega_{r, 0}$ y $\Omega_{\Lambda, 0}$ señalan, respectivamente, la densidad de materia, radiación y vacío, medidas en el tiempo actual $t=t_{0}$, y satisfacen la relación $\Omega_{m}+\Omega_{r}+\Omega_{\Lambda}=1$. La ecuación (3.67) puede escribirse entonces como [164]

$$
\frac{\delta z}{\delta t_{0}}=H_{0}(1+z)\left\{1-\left[\Omega_{m, 0}(1+z)+\Omega_{r, 0}(1+z)^{2}+\Omega_{\Lambda, 0}(1+z)^{-2}\right]^{1 / 2}\right\} .
$$

Notemos que la aproximación de la ecuación (3.78) para fuentes cercanas $(z \ll 1)$ coincide con aquella encontrada a partir de la aproximación covariante presentada en la sección 3.2.

En la figura 3.1 comparamos las curvas cinemática y dinámica del redshift drift para el modelo $\Lambda$ CDM dadas, respectivamente, por las expresiones (3.76) y (3.78). Los valores de los parámetros cinemáticos considerados son $H_{0}=74,2 \pm 3,6 \mathrm{~km} / \mathrm{s} / \mathrm{Mpc}$ [48], $q_{0}=-0,669 \pm 0,052$ [193], $j_{0}=0,284 \pm 0,151$ [193], $\Omega_{\Lambda, 0}=0,725 \pm 0,016$ [52] y $\Omega_{m, 0}=0,2736 \pm 0,0073$ [52]. Podemos observar un buen acuerdo en las curvas para valores $z \lesssim 0,1$, es decir, hasta un valor del redshift para el cual los parámetros cinemáticos, medidos en el tiempo actual, representan una buena aproximación de $a(t)$ [193].

\subsection{Discusión}

Hemos presentado en este capítulo el redshift drift como un test observacional para distinguir entre diferentes modelos cosmológicos. Dada la complejidad que envuelve el cálculo exacto de 


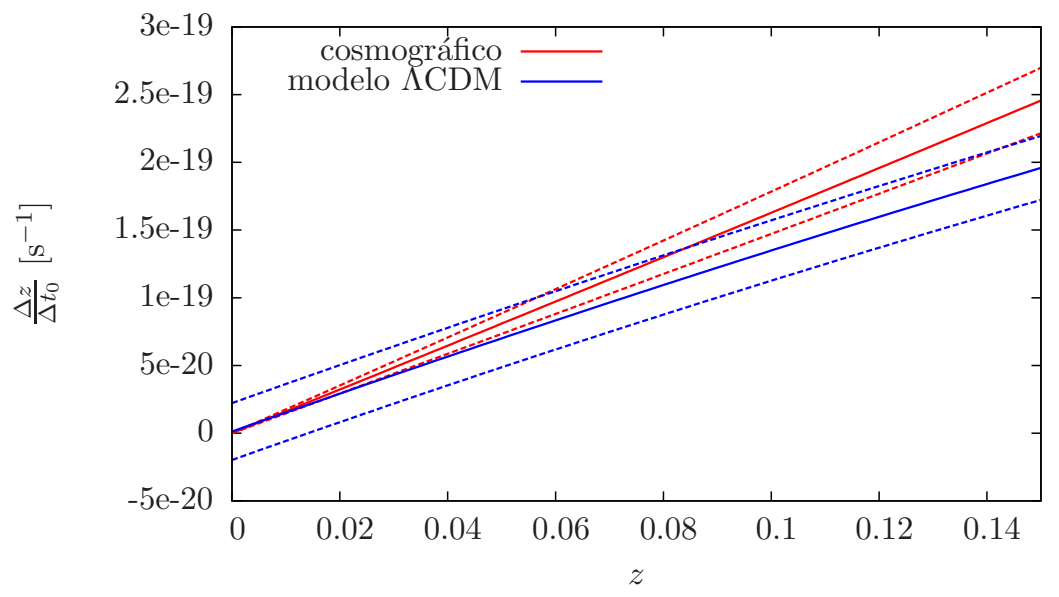

Figura 3.1: Curva dinámica del redshift drift hasta orden 2 para el modelo $\Lambda$ CDM, y su comparación con la curva general obtenida a partir de principios cinemáticos. Las líneas a trazos corresponden a la incerteza en las curvas debidas a la propagación de errores de los parámetros $H_{0}, q_{0}, j_{0}, \Omega_{\Lambda, 0}$ y $\Omega_{m, 0}$.

este observable en geometrías con poca simetría, estudiamos dos aproximaciones válidas para fuentes cercanas.

La primera consiste en un cálculo basado en el formalismo 1+3. Encontramos una fórmula covariante que nos permitió recobrar resultados previamente estudiados en la literatura, pero de una manera considerablemente más sencilla. Para el caso de modelos con geometrías complejas, por otra parte, la aproximación covariante deja en evidencia dependencias angulares que podrían ser utilizadas para optimizar los esfuerzos dedicados a la medición del redshift drift, y estudiar las contribuciones anisótropas en modelos sin simetría esférica. Una aplicación adicional de esta aproximación es la de estudiar modelos anisótropos con rotación no nula [160].

La segunda aproximación, por su parte, está basada en un tratamiento cosmográfico. Es válida para modelos basados en la solución de FLRW, pero no está restringida al campo de la Relatividad General. En esta dirección, presentamos en el apéndice E una aplicación de la aproximación cosmográfica del redshift drift para poner límites sobre el espacio de parámetros de modelos cosmológicos basados en teorías de gravedad modificada $f(R)$.

En la práctica, la comparación de las predicciones teóricas para el redshift drift con los datos observacionales es llevada a cabo a través de la medición del corrimiento espectroscópico en la velocidad de objetos extragalácticos. Dicha cantidad está directamente relacionada con el redshift drift mediante la expresión

$$
\delta v \equiv \frac{c \delta z}{(1+z)} .
$$

Entre los efectos que podrían contaminar la estimación del redshift drift utilizando aproximaciones para fuentes cercanas, podemos mencionar (i) cualquier cambio en la coordenada comóvil de la fuente, debido a una velocidad peculiar no nula, $\delta v$, que modifica la ecuación (3.79) en una cantidad del orden $(\delta v / c) \ll 1$ [164], y (ii) una aceleración peculiar distinta de cero. Por otra parte, además de ser un test directo de la dinámica de la expansión, el redshift 
drift es una cantidad que no depende de la determinación de la luminosidad absoluta de las fuentes, sino de la identificación de determinadas líneas espectrales estables, reduciendo así las incertezas debidas a efectos sistemáticos y/o de evolución [139]. Un análisis detallado de la determinación del redshift drift a partir de la medición de líneas de $21 \mathrm{~cm}$ de HI puede encontrarse en [165].

En lo que respecta a las perspectivas observacionales para determinar el redshift drift, el grado de precisión que se espera alcanzar en los proyectos de grandes telescopios es realmente prometedora. Entre los instrumentos abocados a la medición de este observable dentro de los próximos años, podemos mencionar el European Extremely Large Telescope $(E-E L T)^{8}$, el Thirty Meter Telescope $(T M T)^{9}$, y el Giant Magellan Telescope $(G M T)^{10}$, con diámetros en el rango de 25 a 100 metros. Gracias a la utilización de espectrógrafos estables de alta resolución, se estima que la señal del redshift drift podrá ser medida en pocos años [139]. Debido a que la magnitud de la señal es proporcional al intervalo $\Delta t$ que transcurre entre dos observaciones de la misma fuente, algunas estrategias han sido discutidas para poder reducir el tiempo de medición a un intervalo de entre 10 y 20 años $[88,194]$. En este aspecto, es importante resaltar que no es necesario realizar las observaciones durante todo el intervalo temporal, sino tan sólo al comienzo y al final del período en cuestión [88].

En particular, el espectrógrafo CODEX (COsmic Dynamics Experiment ${ }^{11}$ podría alcanzar la precisión necesaria para detectar la señal del redshift drift a partir del monitoreo del corrimiento de las líneas de absorción Lyman- $\alpha$ de quásares distantes en un período de observación de algunas pocas décadas [195]. Cabe destacar que los quásares, en este sentido, presentan la ventaja de ser fuentes estables y prácticamente no afectadas por movimientos peculiares.

Dada la limitación observacional para detectar desde la Tierra líneas de absorción Lyman$\alpha$ para $z<1,7$ [194], la medición del redshift drift podría efectuarse con otros experimentos futuros, como el interferómetro de ondas gravitacionales DECIGO/BBO. Estos experimentos están basados en la medida de las correcciones debidas a la expansión acelerada del universo sobre la fase de ondas gravitacionales provenientes de sistemas binarios de estrellas de neutrones. La detección de dicha corrección de fase podría ser utilizada para inferir el signo del redshift drift hasta $z$ 0,2 [196]. Con la detección futura de ondas gravitacionales podrían descartarse los modelos de void de LTB, a menos de aquellos con gradientes de sobredensidad a $z 0$ (sin embargo, poco realistas) [139].

A pesar de sus dificultades propias, el método para determinar el redshift drift a partir de la observación de líneas estables de absorción presenta ventajas interesantes [139]:

1. El redshift drift es una prueba directa de la dinámica de la expansión (a diferencia de otros métodos, como aquellos basados en la medición de la distancia de luminosidad, que son esencialmente geométricos). Mismo que la precisión alcanzada no sea la suficiente para poder discriminar entre diferentes modelos cosmológicos, la medición del redshift drift sería incluso importante como una prueba directa de la expansión acelerada, independiente de otras observaciones, y podría utilizarse para testear la dinámica de la expansión esperada en los modelos basados en la Teoría de la Relatividad General en relación a otros escenarios alternativos.

\footnotetext{
${ }^{8}$ https://www.eso.org/sci/facilities/eelt/

${ }^{9}$ http://www.tmt.org/

${ }^{10}$ http://www.gmto.org/

${ }^{11}$ http://ww. iac.es/proyecto/codex/
} 
2. Es importante mencionar que los mapas radiales de las BAO pueden ser también utilizados para medir $H(z)$. Esto es porque las BAO radiales son medidas de distancias comóviles a un dado intervalo de redshift, digamos $z_{*}+\delta z_{*}$, que para intervalos pequeños resulta inversamente proporcional a $H\left(z_{*}\right)$. Sin embargo la medición de las BAO es no trivial y conlleva sus propios errores sistemáticos. Por otra parte, más allá del desafío observacional, el redshift drift es conceptualmente simple, pues no está basado en la calibración previa de velas patrón (como en el caso de las SN tipo Ia), o reglas patrón originadas a partir del crecimiento de perturbaciones (como es el caso de la escala acústica del CMB), o los efectos de clustering de materia (excepto en escalas en donde la aceleración peculiar empieza a jugar un rol dominante). Por lo tanto, el redshift drift puede servir además como un útil cross-check para las $\mathrm{BAO}$ radiales.

3. La observación de quásares distantes impone restricciones sobre la expansión cósmica a $z \gtrsim 2$, en donde las SN tipo Ia y los mapas de grandes estructuras son obtenidos con precisión más baja.

4. El redshift drift es una herramienta que permite distinguir entre aceleración verdadera (es decir, aquella predicha por los modelos de energía oscura) y aceleración aparente (modelos de void).

En relación a la medición de la paralaje cósmica, como observable para distinguir el modelo $\Lambda \mathrm{CDM}$ de modelos anisótropos, satélites de nueva generación como el GAIA ${ }^{12}$, con resolución de $1 \mu$ as, serían capaces de obtener resultados prometedores en el término de 10 años a partir de la observación del orden de 500.000 quásares. Estas observaciones podrían ser utilizadas para distinguir también modelos de universo con rotación no nula. La distinción entre distintos modelos anisótropos, sin embargo, no sería aún viable con esta resolución angular [160].

Por otra parte, dado que los modelos de Stephani presentan shear nulo, una futura detección positiva de la paralaje cósmica podría ser utilizada como otra herramienta observacional para distinguir entre modelos de Stephani y modelos de LTB [176].

Ante estas perspectivas observacionales prometedoras, el estudio teórico de observables a tiempo real como herramienta para distinguir entre modelos cosmológicos cobra cada vez más relevancia. En esta dirección el aporte original presentado en esta tesis da lugar a múltiples aplicaciones y al análisis de modelos cosmológicos con geometrías complejas.

\footnotetext{
${ }^{12}$ http://sci.esa.int/gaia/
} 
"Se formó una Sociedad con el nombre de LOS LIMPIADORES DE ESTRELLAS. Era suficiente llamar al teléfono 50-4765 para que de inmediato salieran las brigadas de limpieza, provistas de todos los implementos necesarios y muñidas de órdenes efectivas que se apresuraban a llevar a la práctica; tal era, al menos, el lenguaje que empleaba la propaganda de la Sociedad. En esta forma, bien pronto las estrellas del cielo readquirieron el brillo que el tiempo, los estudios históricos y el humo de los aviones habían empañado. (...)"

Los limpiadores de estrellas

Prolegómenos de la Astronomía La otra orilla (1937-1945)

Julio Florencio Cortázar 


\section{Capítulo 4}

\section{Inflación y regiones no homogéneas}

Como mencionamos en el capítulo 1, un ingrediente importante en el modelo cosmológico estándar es la inflación, propuesta para resolver, entre otros, los problemas del horizonte y de la curvatura plana.

Una de las hipótesis fundamentales presente en la mayoría de los modelos inflacionarios es la de suponer un espacio-tiempo homogéneo e isótropo como condición inicial para que el proceso de inflación tenga lugar. Este escenario se justifica comúnmente considerando que, de haber existido regiones no homogéneas previas a la etapa inflacionaria, éstas deberían haber sido diluidas durante la expansión exponencial típica de estos modelos. Si bien existen argumentos que apoyan este razonamiento, no hay hasta el momento una prueba definitiva de que el proceso de inflación tenga efectivamente la propiedad de eliminar cualquier región no homogénea en la distribución de materia primordial. Es entonces importante abordar los problemas relacionados con el comienzo y de la evolución del proceso de inflación en una región con materia, con o sin presión, y, en particular, con distribución no homogénea.

El problema de la evolución del proceso inflacionario bajo condiciones iniciales generales ha sido estudiado por varios autores proponiendo un escenario simplificado que contempla la evolución de una región de vacío con constante cosmológica no nula, inmersa en un espaciotiempo con materia sin presión, tanto para los casos homogéneos como para aquellos casos con distribución radial no uniforme de materia. Tales regiones son descriptas, respectivamente, por las métricas de FLRW y de LTB. La superficie de separación entre ambas está modelada por una cáscara delgada con densidad de materia no nula, que satisface las condiciones de pegado de Israel. La motivación que lleva a estudiar la evolución dinámica de estas configuraciones es poder determinar si la expansión de la región de vacío podría inhibirse a causa de un contenido de materia genérico en el ambiente.

Presentaremos en este capítulo una generalización del problema mencionado anteriormente para el caso en el que la región externa es descripta por una solución con contenido de materia de radiación, es decir, con presión no nula. Tal escenario será modelado con las soluciones de FLRW y de Lemaître, para los casos homogéneo y con distribución radial no uniforme, respectivamente. El objetivo de este trabajo es estudiar si los efectos de la presión de la radiación pueden alterar el proceso de expansión que proponen los modelos inflacionarios. 


\subsection{Modelos inflacionarios}

La inflación cosmológica es una fase de expansión exponencial (no adiabática) que habría tenido lugar durante las etapas de evolución más tempranas del universo, inmediatamente después de que éste emergiera de la llamada era de Planck, dominada por procesos de gravedad cuántica.

La existencia de una etapa inflacionaria fue propuesta originalmente por Guth en el año 1981 [197] con el fin de solucionar, entre otras cosas, algunos de los problemas del modelo cosmológico estándar, como el del horizonte y el de la curvatura plana. ${ }^{1}$ Este escenario de inflación, conocido hoy en día como "inflación antigua", fue sugerido como una posible consecuencia de ciertas transiciones de fase, existentes en el marco de las GUT. En términos simples, el modelo de inflación antigua proponía el surgimiento de regiones del espacio-tiempo con expansión exponencial del factor de escala durante la época más temprana de la evolución del universo $\left(T \simeq 10^{17} \mathrm{GeV}\right) .{ }^{2}$ Estas regiones podían ser modeladas como "burbujas", con un contenido de densidad de energía con ecuación de estado $p=-\rho$, esto es, con una dinámica descripta por la solución de de Sitter. Así, la expansión exponencial durante el proceso de inflación conectaba de forma causal todo el universo observable hoy en día. El escenario de inflación antigua presentaba, sin embargo, serios problemas para describir una "salida elegante" de la fase de inflación sin que el espacio-tiempo perdiera la condición de homogeneidad [63]. Más tarde, fueron propuestos los llamados modelos de "inflación nueva" (1982) [198, 199, 200, 201, 202], "inflación caótica" (1983) [203, 204, 205, 206] e "inflación eterna" [207].

Actualmente, el paradigma de inflación está basado en la existencia de un campo escalar masivo acoplado mínimamente a la gravedad, que habría sufrido un desplazamiento de su posición de equilibrio (correspondiente al mínimo de su potencial) durante la época posterior a la fase de Planck en la evolución más temprana del universo. La dinámica de dicho campo es descripta por lo que conocemos como evolución de "caída lenta" (o slow rolling) hacia el mínimo de su potencial, es decir, cuando el término del potencial domina sobre los términos cinético y de curvatura en la ecuación de evolución del campo escalar. Bajo estas condiciones, si durante la etapa inflacionaria el término del potencial es elegido de forma tal que su variación sea prácticamente nula, el fluido descripto por campo escalar masivo juega el papel de una constante cosmológica efectiva, que da lugar a una fase de expansión acelerada en la evolución del universo [63].

\subsubsection{El mecanismo básico de la inflación}

Varios de los problemas que presenta el modelo cosmológico estándar, mencionados en la sección 1.2, pueden ser resueltos si suponemos que el universo emerge de la era de Planck con ciertas condiciones iniciales "naturales", tales que den lugar rápidamente a una etapa de evolución descripta por la solución de de Sitter. Durante esta etapa, la dinámica del factor de escala atraviesa una fase de expansión exponencial que, como veremos a continuación, ofrece una solución simple a algunos de los aspectos problemáticos del escenario cosmológico.

\footnotetext{
${ }^{1}$ La inflación no es la única solución posible: un "rebote" (o bounce) en el que el factor de expansión pasa por un mínimo también podría resolver tales problemas [67].

${ }^{2} \mathrm{~A}$ escalas temporales menores, equivalentes a temperaturas $T \simeq M_{P l} \equiv 1,22 \times 10^{19} \mathrm{GeV}$, sería necesario un tratamiento cuántico para describir adecuadamente a la gravitación. El problema de valores iniciales para trabajar con soluciones exactas de las ecuaciones de Einstein puede ser aplicado sólo si la temperatura del fluido cosmológico satisface $T<<M_{P l}$ [197].
} 
Para modelar un universo con una etapa de expansión de de Sitter es necesario la aparición de una constante cosmológica efectiva, que deje de actuar después de un cierto intervalo de tiempo, para dar luego lugar a las etapas dinámicas dominadas, sucesivamente, por radiación y por materia. En los modelos inflacionarios, dicha constante cosmológica es generada a partir de un estado de falso vacío de un campo escalar masivo primordial bajo la acción de un potencial $V(\phi) \simeq$ cte. $^{3}$ El modelo debe ser tal que la propia evolución del campo escalar haga que el término asociado al potencial, dominante al comienzo, decrezca luego de un cierto intervalo de tiempo y, en consecuencia, el universo pueda salir de la etapa inflacionaria. Es decir que los modelos inflacionarios deben proveer un mecanismo efectivo para que el universo entre y salga de la etapa de expansión acelerada. Asimismo, al final de la inflación debe producirse una etapa de recalentamiento $[198,217,218]$, durante la cual el campo escalar disipa su energía oscilando en torno a la posición de potencial mínimo, para dar lugar a la creación de partículas relativistas [63].

El tensor de energía-momento que caracteriza a un campo escalar masivo (acoplado mínimamente a la gravedad) se expresa como [63]

$$
T_{\mu \nu}=\nabla_{\mu} \phi \nabla_{\nu} \phi-\frac{1}{2} g_{\mu \nu}\left[g^{\alpha \beta} \nabla_{\alpha} \phi \nabla_{\beta} \phi+V(\phi)\right] .
$$

En un fondo homogéneo e isótropo, descripto por la métrica de FLRW, $T_{\mu \nu}$ toma la forma de un fluido perfecto con densidad de energía y presión dadas, respectivamente, por

$$
\begin{aligned}
& \rho \equiv T_{00}=\frac{1}{2} \dot{\phi}^{2}+\frac{\nabla^{2} \phi}{2 a^{2}}+V(\phi) \equiv \rho_{\dot{\phi}}+\rho_{\text {grad }}+V, \\
& p \equiv \frac{1}{3} \sum_{i} T_{i i}=\frac{1}{2} \dot{\phi}^{2}-\frac{\nabla^{2} \phi}{6 a}-V(\phi) \equiv \rho_{\dot{\phi}}-\frac{1}{3} \rho_{\text {grad }}-V,
\end{aligned}
$$

en donde $\rho_{\dot{\phi}}$ y $\rho_{\text {grad }}$ son las densidades de energía asociadas a los términos cinético y de gradiente de $\phi$, respectivamente. Si el término del potencial $V$ es significativamente mayor que los dos anteriores, la ecuación de estado para la materia del campo resulta $\rho \simeq-p \simeq$ $-V(\phi) \simeq$ cte. Luego, $\phi$ también permanece aproximadamente constante durante ese período de tiempo (o, para ser más precisos, $\phi$ varía mucho más lentamente que la tasa de expansión del universo, dada por $H(t) \equiv \dot{a} / a)$. El factor de escala entra entonces en una fase de de Sitter, con la siguiente dependencia temporal

$$
a(t) \simeq a_{0} \exp \left(\int_{t_{0}}^{t_{e}} \sqrt{\frac{8 \pi \bar{\rho}}{3 m_{p l}^{2}}} \mathrm{~d} t\right) \simeq a_{0} \exp \left(\int_{t_{0}}^{t_{e}} \sqrt{\frac{8 \pi V(\bar{\phi})}{3 m_{p l}^{2}}} \mathrm{~d} t\right),
$$

en donde $t_{e} \mathrm{y} t_{0}$ señalan, respectivamente, los tiempos inicial y final de la etapa inflacionaria, y $\bar{\rho}$ y $V(\bar{\phi})$ indican cantidades aproximadamente constantes. El parámetro de Hubble durante este período resulta [63]

$$
H(t)=\sqrt{\frac{8 \pi V(\bar{\phi})}{3 m_{p l}^{2}}} .
$$

\footnotetext{
${ }^{3}$ La existencia de una fase de falso vacío, en el contexto de las GUT, que diera lugar a una etapa de expansión descripta por la solución de de Sitter había sido investigada previamente en diversos trabajos [208, 209, 210, 211, 212, 213, 214, 215, 216]. Sin embargo, fue Guth en el año 1981 [197] el primero en llamar "inflación" a esta etapa de evolución del universo y señalar en forma precisa las soluciones concretas que ofrecía a la mayoría de los problemas que el modelo estándar presentaba [63].
} 
Con este escenario, es posible entonces dar solución a algunos de los problemas del modelo cosmológico (sección 1.2). Dado que el factor de escala crece exponencialmente, al final de la etapa inflacionaria el término de curvatura $k / a^{2}$ en la ecuación de evolución (1.6) puede ser despreciado, y el problema de la curvatura plana queda de este modo resuelto. Por otra parte, el tamaño físico del horizonte de partículas crece durante la etapa inflacionaria como

$$
H_{\text {partícula }}^{-1} \equiv a\left(t_{e}\right) \int_{t_{0}}^{t_{e}} \frac{\mathrm{d} t}{a(t)} \simeq \frac{\mathrm{e}^{H \Delta t}}{H} .
$$

El crecimiento del horizonte es entonces lo suficientemente rápido como para justificar que las regiones del universo observable actualmente hayan estado confinadas a un dominio pequeño con contacto causal antes de la etapa de inflación, solucionando así el problema del horizonte. Asimismo, dado que durante la inflación las fluctuaciones cuánticas del campo escalar resultan independientes de escala, el modelo inflacionario provee también las semillas que darán lugar a la formación de estructuras en las etapas posteriores de la evolución del universo [219].

\subsubsection{Condiciones iniciales para la inflación}

A pesar de que la inflación cosmológica ha sido especialmente desarrollada para resolver problemas concretos del modelo cosmológico estándar, no está exenta de presentar ciertas dificultades propias. Existen actualmente dos cuestiones importantes aún no resueltas en los modelos de inflación:

1. cómo compatibilizar los escenarios de inflación con un modelo realista de partículas elementales, $\mathrm{y}$

2. determinar si el surgimiento de la etapa inflacionaria es genérica o necesita de condiciones iniciales especiales, así como también estudiar si el proceso de inflación puede evolucionar en escenarios arbitrarios (en particular, con una distribución de materia no homogénea).

En relación al primer punto, el paradigma de inflación debe estar en acuerdo con los modelos de física de partículas aceptados hoy en día [220]. En esta dirección, existen en la literatura algunos modelos que proponen la existencia de campos escalares y partículas que podrían representar al "inflatón", aunque este es un tema que se encuentra aún en discusión [220, 221].

En el contexto de esta tesis, nos interesa sin embargo profundizar en el problema de las condiciones iniciales necesarias para que una etapa inflacionaria haya tenido lugar en al evolución cósmica. Si bien uno de los principales objetivos de la inflación es explicar la homogeneidad a grandes escalas en el universo (es decir, dar un argumento sólido a los modelos cosmológicos basados en la métrica homogénea e isótropa de FLRW), un ingrediente común en todos los modelos de inflación basados en la Teoría de la Relatividad General es la hipótesis de homogeneidad e isotropía primordiales para el comienzo de la propia inflación. Dicha hipótesis se basa en la comúnmente llamada conjetura cosmológica de "no hair" establece que los espacio-tiempos descriptos por la métrica de de Sitter son soluciones asintóticamente estables de las ecuaciones de Einstein si existe una constante cosmológica efectiva positiva [222, 223, 224]. Como consecuencia, una vez empezado el proceso de inflación, cualquier región no homogénea es suavizada por la expansión acelerada. Sin embargo, este resultado no siempre es aplicable durante la etapa pre-inflacionaria. En esta dirección, existen trabajos que estudian el comienzo de inflación en escenarios homogéneos pero anisótropos [225], y en escenarios no 
homogéneos [226]. Estos últimos modelan, sin embargo, las regiones no homogéneas como perturbaciones de la solución homogénea de FLRW, y no a partir de soluciones no homogéneas más generales, como la solución de LTB o la solución de Lemaître.

El problema que plantea la evolución de un universo descripto por la métrica de FLRW y un campo escalar no homogéneo como fuente fue estudiado en detalle en [63], dedicando particular atención al rol que juegan los términos cinético y de curvatura en la evolución dinámica del campo con condiciones iniciales no homogéneas. En el contexto de escenarios de inflación "caótica", los autores de dicho trabajo encuentran que la etapa de inflación es genérica, mientras que para los modelos de "nueva" inflación las condiciones iniciales que dan lugar a una transición de fase en el marco de las GUT son específicas y poco generales. Por otra parte, en lo que respecta a escenarios no homogéneos, otros trabajos numéricos [227, 228, 229] y analíticos [230] han mostrado que es estrictamente necesario suponer para los campos escalares primordiales condiciones iniciales de homogeneidad e isotropía en alto grado para que la etapa de inflación pueda tener lugar.

La evolución de una burbuja en un fondo de vacío ha sido estudiada ampliamente en la literatura para distintos valores de las constantes cosmológicas que caracterizan cada región [231, 232, 233, 234, 235]. La generalización del formalismo de Israel para el caso de una cáscara delgada con presión nula expandiéndose en un fondo homogéneo con polvo como fuente fue presentada en [236]. La integración numérica del problema relativista completo muestra como resultado que todas las burbujas de vacío inicialmente menores que el tamaño del horizonte se expanden indefinidamente. Para el caso particular de modelos de universo abierto, el crecimiento del radio de la burbuja puede eventualmente frenarse, mientras que en universos con curvatura nula, la burbuja de vacío de expande más rápido que el fondo y su radio alcanza valores asintóticos. La expansión es aún mucho más rápida para el caso de universos cerrados, y la cáscara eventualmente alcanza velocidades cercanas a la velocidad de la luz [237].

El problema que plantea la evolución de una burbuja en un fondo con distribución de materia no homogénea fue estudiado en [234, 235, 238]. En estos trabajos, los autores muestran que la evolución de la región de vacío que encierra la burbuja no se inhibe por causa del contenido de materia en la región externa.

Sin embargo, todos los trabajos mencionados anteriormente están limitados a estudiar el caso de burbujas evolucionando en fondos con contenido de materia con presión nula, es decir, polvo. Ante estos resultados, nos propusimos abordar en el marco de esta tesis el estudio de la evolución del proceso de inflación en escenarios arbitrarios. En particular, nos interesa investigar la dinámica de regiones de vacío en fondos no homogéneos, para analizar si condiciones iniciales generales podrían inhibir el proceso inflacionario. Una manera simple de encarar este problema es modelar las regiones inflacionarias de vacío como "burbujas" con simetría esférica. Supondremos que la nucleación de estas burbujas tiene lugar en espaciotiempos con contenido de materia no homogéneo, descriptos por las soluciones de LTB o de Lemaître. La separación entre la burbuja y el fondo no homogéneo, por su parte, puede ser modelada con una cáscara delgada (esto es, con paredes esféricas de espesor mucho menor que el radio de la región que encierran). Bajo estas simplificaciones, el análisis queda reducido entonces a estudiar la dinámica de la cáscara delgada que separa ambas regiones, imponiendo las condiciones de pegado de Israel [239], a través de la superficie de separación, para las soluciones de las ecuaciones de Einstein que modelan cada región. Esto es lo que conocemos como formalismo de cáscara delgada o thin shell. 
Dado que es esperable que las condiciones primordiales del universo den lugar a presencia de materia relativista, focalizaremos nuestro trabajo en estudiar la evolución de regiones de vacío en fondos con contenido de materia de radiación, tanto con distribuciones homogéneas cuanto no homogéneas. La evolución numérica será computada mediante la implementación de un código que integre adecuadamente el sistema de ecuaciones diferenciales parciales correspondiente. Las condiciones iniciales que estudiaremos son características de los modelos inflacionarios, y los parámetros para describir la geometría contemplarán escenarios cosmológicos genéricos.

\subsection{El formalismo de la cáscara delgada}

El formalismo de cáscara delgada (también conocido como formalismo de condiciones de juntura o formalismo de thin shell) es una técnica desarrollada para estudiar problemas de Relatividad General que involucren campos gravitacionales producidos por una lámina o cáscara delgada de materia [239, 240, 241, 242, 243, 244]. El campo gravitacional generado por dicha cáscara es descripto por una métrica continua, pero no derivable en la región ocupada por la cáscara. Con esto, la conexión afín (símbolos de Christoffel) resultan funciones con discontinuidades tipo escalón, mientras que el tensor de Riemann, por su parte, toma la forma de una función delta de Dirac [245]. Aunque el campo de aplicabilidad del formalismo es amplio, para los propósitos de nuestro trabajo nos concentraremos en describir un escenario con simetría esférica y una cáscara delgada tipo tiempo (esto es, ortogonal a las hipersuperficies con $t=$ cte) ${ }^{4}$

El modelo de cáscara delgada supone la existencia de dos regiones de espacio-tiempo, $\mathcal{M}^{-}$ y $\mathcal{M}^{+}$, caracterizadas cada una por una métrica y un contenido de materia particulares. Las regiones están separadas por una hipersuperficie con simetría esférica, $\Sigma$ (ver figura 4.1) [246]. La superficie de separación es modelada con una cáscara delgada (de radio despreciable), caracterizada por un tensor energía-momento y una ecuación de estado particular para describir su contenido de materia. Las regiones y la cáscara están unidas mediante las condiciones de pegado de Israel [231, 239, 245, 247], las cuales permiten relacionar la discontinuidad en la curvatura externa de la cáscara en cada una de las regiones, con su tensor energía-momento en la superficie de separación $\Sigma$. El formalismo de cáscara delgada ha sido previamente utilizado en la literatura para describir la evolución de burbujas cósmicas en el contexto de modelos inflacionarios [231, 234, 235]. El esquema simplifica el problema más complejo de modelar la transición entre ambas regiones con un perfil gradual ppara la cáscara, y ofrece una buena aproximación para estudiar el escenario cosmológico que motiva nuestro trabajo.

Con el fin de analizar la evolución de burbujas cósmicas en el contexto de escenarios de inflación, nos limitaremos a estudiar el formalismo de cáscara delgada para el caso de una región interna homogénea de vacío, y una región externa no homogénea y con simetría esférica. El modelo queda caracterizado entonces de la siguiente manera [234]:

- Una región interna de vacío, $\mathcal{M}^{-}$, con constante cosmológica $\Lambda_{-}$, y descripta por una métrica isótropa y homogénea, con elemento de línea expresado en las coordenadas

\footnotetext{
${ }^{4}$ Un ejemplo simple, análogo al caso gravitacional, aparece en el problema de electrostática definido por una lámina cargada, con densidad de carga $\rho(\vec{x})=\sigma \delta(z)$. La densidad superficial de carga, $\sigma$, generará un potencial eléctrico continuo, $\phi(\vec{x})=\vec{E}_{0} \cdot \vec{x}+|z| \sigma / \epsilon_{0}$, y una discontinuidad en el campo eléctrico, $\vec{E}(\vec{x})=\vec{E}_{0}+\operatorname{sign}(z) \hat{z} \sigma / \epsilon_{0}$ $[245]$.
} 


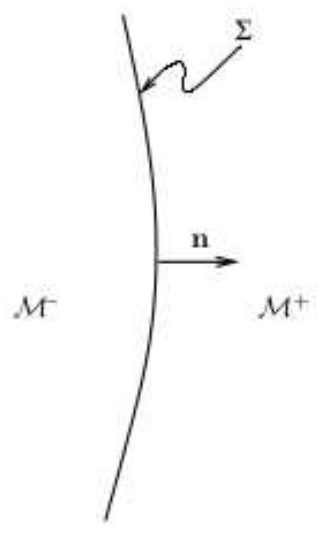

Figura 4.1: (extraída de [246]) Modelo de cáscara delgada o thin shell: dos regiones del espaciotiempo, $\mathcal{M}^{-}$y $\mathcal{M}^{+}$, separadas por una hipersuperficie $\Sigma$ con simetría esférica y orientada con el vector $\mathbf{n}$.

internas $(T, z, \theta, \phi)$ como

$$
\left.\mathrm{d} s^{2}\right|_{\mathcal{M}^{-}}=g_{\mu \nu}^{-} \mathrm{d} x_{-}^{\mu} \mathrm{d} x_{-}^{\mu}=\mathrm{d} T^{2}-b^{2}(T)\left(\frac{\mathrm{d} z^{2}}{1+z^{2}}+\mathrm{d} \Omega^{2}\right) .
$$

La evolución del factor de escala queda determinada por la ecuación

$$
\left(\frac{\mathrm{d} b}{\mathrm{~d} T}\right)=\left(\frac{\Lambda_{-}}{3}\right) b^{2}(T)+1 .
$$

- La hipersuperficie de separación $\Sigma$, que describe la evolución de la burbuja con simetría esférica, está caracterizada por el elemento de línea

$$
\left.\mathrm{d} s^{2}\right|_{\Sigma}=g_{a b} \mathrm{~d} x^{a} \mathrm{~d} x^{b}=\mathrm{d} \tau^{2}-\rho(\tau)^{2} \mathrm{~d} \Omega^{2},
$$

en donde $(\tau, \theta, \phi)$ son las coordenadas comóviles sobre la burbuja. Supondremos que la misma puede describirse como una cáscara delgada de fluido perfecto, con tensor energía-momento dado por

$$
T_{\mu \nu}^{\Sigma}=\left[\sigma(\tau)+p_{\sigma}(\tau)\right] u_{\mu}^{\Sigma} u_{\nu}^{\Sigma}-p_{\sigma}(\tau) g_{\mu \nu}^{\Sigma},
$$

y una ecuación de estado de la forma $p_{\sigma}=w \sigma$.

- La región externa, $\mathcal{M}^{+}$, queda caracterizada por la solución de Lemaître en coordenadas externas $(t, r, \theta, \phi)$, con elemento de línea dado por

$$
\left.\mathrm{d} s^{2}\right|_{\mathcal{M}^{+}}=g_{\mu \nu}^{+} \mathrm{d} x_{+}^{\mu} \mathrm{d} x_{+}^{\mu}=\mathrm{e}^{A(t, r)} \mathrm{d} t^{2}-\mathrm{e}^{B(t, r)} \mathrm{d} r^{2}-R^{2}(t, r) \mathrm{d} \Omega^{2},
$$

y un fluido perfecto como fuente, con tensor de energía-momento

$$
T_{\mu \nu}^{+}=[\epsilon(t, r)+p(t, r)] u_{\mu}^{+} u_{\nu}^{+}-p(t, r) g_{\mu \nu}^{+}-\Lambda_{+} g_{\mu \nu}^{+} .
$$


Recordemos que la métrica de Lemaître es la solución más general posible para describir regiones no homogéneas con simetría esférica y un fluido perfecto con ecuación de estado del tipo $p(t, r)=\alpha \epsilon(t, r)$. Como veremos en las secciones subsiguientes, las soluciones de FLRW y de LTB pueden recuperarse a partir de una elección adecuada de $\rho(t, r)$ y $p(t, r)$. El caso particular de una región externa descripta por la métrica de LTB (esto es, con polvo no homogéneo como fuente) puede recuperarse imponiendo la ecuación de estado $p(t, r)=0$, mientras que el límite homogéneo para describir una evolución tipo FLRW tiene lugar eligiendo $(i) \epsilon(t, r)=\epsilon(t)$ y $p(t, r)=p(t)=\frac{1}{3} \epsilon(t)$, para un fluido de radiación, y $(i i) \epsilon(t, r)=\epsilon(t)$ y $p(t, r)=0$ para el caso de un fluido sin presión.

En adelante, los subíndices "-" y "+" indicarán cantidades en las regiones interna y externa, respectivamente. Es importante notar que las métricas están expresadas en diferentes sistemas coordenados, característicos de cada geometría. Las coordenadas angulares de cada región coinciden debido a la simetría esférica del problema.

El pegado continuo de las dos regiones a través de la cáscara delgada impone las siguientes restricciones sobre las funciones métricas:

$$
b(T, \zeta)=\rho(\tau)=R(t, r),
$$

y

$$
\mathrm{d} T^{2}-\left(\frac{b^{2}(T)}{1+\zeta^{2}}\right) \mathrm{d} \zeta^{2}=\mathrm{d} \tau^{2}=\mathrm{e}^{A(t, r)} \mathrm{d} t^{2}-\mathrm{e}^{B(t, r)} \mathrm{d} r^{2} .
$$

Las ecuaciones (4.13) y (4.14) permiten expresar $(T, \zeta)$ y $(t, r)$ como funciones $(T(\tau), \zeta(\tau))$ y $(t(\tau), r(\tau))$. Es decir, que la evolución de la cáscara puede ser, o bien estudiada en términos de las coordenadas que caracterizan las regiones interna o externa, o bien parametrizada por la coordenada temporal $\tau$ sobre la burbuja. Dado que la solución que caracteriza a la región externa es, en general, compleja y conocida sólo numéricamente, la descripción del problema será realizada utilizando un sistema de referencia comóvil con las coordenadas externas $(t, r, \theta, \phi)$. Notemos que, si parametrizamos la evolución de la cáscara delgada con la coordenada temporal $t$ de la región externa, entonces sobre la hipersuperficie $\Sigma$ tenemos $\tau=\tau(t)$ y $r=r(t)$. En adelante, denotaremos con la función $x(t)$ a la coordenada radial externa de la burbuja, esto es, $\left.x(t) \equiv r\right|_{\Sigma}=\left.r(t)\right|_{\Sigma}$.

Teniendo en cuenta las restricciones (4.13) y (4.14), las condiciones de pegado de Israel resultan [231, 239, 246, 247]:

$$
\begin{aligned}
-\frac{\sigma}{2} & =\left[K_{\theta}^{\theta}\right], \\
p_{\sigma} & =\left[K_{\tau}^{\tau}\right]+\left[K_{\theta}^{\theta}\right], \\
\frac{\mathrm{d} \sigma}{\mathrm{d} \tau}+\frac{2}{\rho} \frac{\mathrm{d} \rho}{\mathrm{d} \tau}\left(\sigma+p_{\sigma}\right) & =-\left[T_{\tau}^{n}\right],
\end{aligned}
$$

en donde todas las cantidades son funciones del tiempo propio de la burbuja, $\tau$, y los corchetes aplicados a una dada cantidad $A$ indican la diferencia $[A]=A^{+}-A^{-}$. El tensor $K_{a b} \equiv$ $n_{\alpha ; \beta} \mathbf{e}_{a}^{\alpha} \mathbf{e}_{b}^{\beta}$ es la curvatura extrínseca de la cáscara, y $T_{\tau}^{n} \equiv \mathbf{e}_{\tau}^{\alpha} T_{\alpha}^{\beta} n_{\beta}$ es la proyección normal a la superficie de la burbuja del tensor energía-impulso. Las ecuaciones (4.15)-(4.17) determinan la evolución del radio $\rho$ de la cáscara, y de su densidad de materia $\sigma$, y están acopladas a la evolución de la geometría de las regiones interna y externa (estas últimas dadas por las ecuaciones de Einstein). En particular, la ecuación (4.16) puede ser reemplazada por una 
ecuación de estado para el contenido de materia sobre la cáscara, que supondremos de la forma $p_{\sigma}=w \sigma$.

Para estudiar la evolución de la burbuja nos resta sólo reescribir las ecuaciones (4.15) y (4.17) en términos de las coordenadas de la métrica externa, como detallamos a continuación. Para ello, tenemos enn cuenta que los proyectores sobre la hipersuperficie $\Sigma$, expresados en las coordenadas externas, son

$$
\begin{aligned}
\mathbf{e}_{\tau}^{\alpha} & =\left(\frac{\mathrm{d} t}{\mathrm{~d} \tau}, \frac{\mathrm{d} x}{\mathrm{~d} \tau}, 0,0\right), \\
\mathbf{e}_{\theta}^{\alpha} & =(0,0,1,0), \\
\mathbf{e}_{\phi}^{\alpha} & =(0,0,0,1) .
\end{aligned}
$$

La velocidad de la burbuja es $u^{\alpha}=\mathbf{e}_{\tau}^{\alpha}$ y el vector normal orientado hacia la región externa queda definido por las condiciones $u^{\alpha} n_{\alpha}=0$ y $n^{\alpha} n_{\alpha}=-1$. En particular, para la métrica externa que estamos considerando resulta

$$
n_{\alpha}=\gamma_{+} \mathrm{e}^{A(t, x) / 2} \mathrm{e}^{B(t, x) / 2}\left(-\left(\frac{\mathrm{d} x}{\mathrm{~d} \tau}\right),\left(\frac{\mathrm{d} t}{\mathrm{~d} \tau}\right), 0,0\right),
$$

en donde el parámetro $\gamma_{+}$indica si la burbuja está en expansión $\left(\gamma_{+}=1\right)$ o en contracción $\left(\gamma_{+}=-1\right)$. Los proyectores y el vector normal expresados en las coordenadas internas son definidos en forma análoga.

Con las definiciones anteriores, las componentes angulares del tensor de curvatura extrínseca resultan

$$
\begin{aligned}
& K_{a b}^{-} \equiv n_{\alpha ; \beta}^{-}\left(\mathbf{e}_{a}^{\alpha}\right)^{-}\left(\mathbf{e}_{b}^{\beta}\right)^{-}=\gamma_{-} \frac{1}{\rho \sqrt{1+\zeta^{2}}}\left(\zeta b \frac{\mathrm{d} z}{\mathrm{~d} \tau} \frac{\mathrm{d} b}{\mathrm{~d} T}+\left(1+z^{2}\right) \frac{\mathrm{d} T}{\mathrm{~d} \tau}\right), \\
& K_{a b}^{+} \equiv n_{\alpha ; \beta}^{+}\left(\mathbf{e}_{a}^{\alpha}\right)^{+}\left(\mathbf{e}_{b}^{\beta}\right)^{+}=\gamma_{+} \frac{1}{\rho} \mathrm{e}^{-A / 2} \mathrm{e}^{-B / 2}\left(\mathrm{e}^{A} R^{\prime} \frac{\mathrm{d} t}{\mathrm{~d} \tau}+\mathrm{e}^{B} \dot{R} \frac{\mathrm{d} x}{\mathrm{~d} \tau}\right),
\end{aligned}
$$

en donde $\gamma_{-}=1\left(\gamma_{-}=-1\right)$ indica que el radio expresado en coordenadas internas está creciendo (decreciendo). Luego, la ecuación (4.15) puede ser reescrita de la siguiente forma [234]

$$
\gamma_{+} \sqrt{\left(\frac{\mathrm{d} \rho}{\mathrm{d} \tau}\right)^{2}-\Delta_{+}}-\gamma_{-} \sqrt{\left(\frac{\mathrm{d} \rho}{\mathrm{d} \tau}\right)^{2}-\Delta_{+}}=-\frac{\sigma \rho}{2}
$$

con

$$
\begin{aligned}
& \Delta_{+}=\frac{\dot{R}^{2}}{\mathrm{e}^{A}}-\frac{R^{\prime 2}}{\mathrm{e}^{B}}=-1+\left(\frac{2 M}{R^{3}}+\frac{\Lambda_{+}}{3}\right) \rho^{2}, \\
& \Delta_{-}=-\left(1+\zeta^{2}\right)+\zeta^{2}\left(\frac{\mathrm{d} b}{\mathrm{~d} T}\right)^{2}=-1+\frac{\Lambda_{-}}{3} \rho^{2},
\end{aligned}
$$

en donde $M(t, r)$ es una función de integración presente en la solución de Lemaître, definida por la ecuación (2.46). Trabajando con la segunda igualdad de las expresiones (4.25) y (4.26), la ecuación (4.24) puede reescribirse de la forma [234]

$$
\left(\frac{\mathrm{d} \rho}{\mathrm{d} \tau}\right)^{2}=\rho^{2} V^{2}-1
$$


con

$$
V^{2}=\Lambda_{-}+\left[\frac{\sigma}{4}+\frac{1}{\sigma}\left(\frac{\Lambda_{+}-\Lambda_{-}}{3}+\frac{2 M}{R^{3}}\right)\right]^{2} .
$$

Teniendo en cuenta que sobre la burbuja $\rho(\tau)=R(t, x)$, y reemplazando $(\mathrm{d} \rho / \mathrm{d} \tau)$ por la expresión (4.27), la ecuación (4.15) resulta

$$
\left(\frac{\mathrm{d} x}{\mathrm{~d} t}\right)^{2}\left[R^{\prime 2}+\mathrm{e}^{B}\left(R^{2} V^{2}-1\right)\right]+2 \dot{R} R^{\prime}\left(\frac{\mathrm{d} x}{\mathrm{~d} t}\right)+\dot{R}^{2}-\mathrm{e}^{A}\left(R^{2} V^{2}-1\right)=0 .
$$

Notemos que ahora todas las funciones involucradas están expresadas en términos de las coordenadas externas de la burbuja $(t, x(t))$.

Nos resta reescribir también la ecuación (4.15) en coordenadas $(t, x(t))$. La proyección normal a la superficie de la burbuja del tensor energía-impulso externo es

$$
\left(T_{\tau}^{n}\right)^{+} \equiv \mathbf{e}_{\tau}^{\alpha} T_{\alpha}^{\beta} n_{\beta}=-\gamma\left(\frac{\mathrm{d} t}{\mathrm{~d} \tau}\right)\left(\frac{\mathrm{d} x}{\mathrm{~d} \tau}\right) \frac{\mathrm{e}^{A(t, x) / 2} \mathrm{e}^{B(t, x) / 2}[\epsilon(t, x)+p(t, x)]}{\sqrt{\mathrm{e}^{A(t, x)}-\mathrm{e}^{B(t, x)}\left(\frac{\mathrm{d} x}{\mathrm{~d} t}\right)^{2}}} .
$$

Por otra parte, dado que la burbuja encierra una región de vacío, resulta $\left(T_{\tau}^{n}\right)^{-}=0$. Las ecuaciones de evolución para la burbuja resultan entonces

$$
\begin{aligned}
\frac{\mathrm{d} x}{\mathrm{~d} t} & =\frac{-\dot{R} R^{\prime} \pm \sqrt{\left(R^{2} V^{2}-1\right)\left[R^{\prime 2} \mathrm{e}^{A}-\dot{R}^{2} \mathrm{e}^{B}+\mathrm{e}^{A} \mathrm{e}^{B}\left(R^{2} V^{2}-1\right)\right]}}{R^{\prime 2}+\mathrm{e}^{B}\left(R^{2} V^{2}-1\right)}, \\
\frac{\mathrm{d} \sigma}{\mathrm{d} t} & =-2\left(\sigma+p_{\sigma}\right) \frac{\dot{R}}{R}+\gamma_{+}(\epsilon+p)\left(\frac{\mathrm{d} x}{\mathrm{~d} t}\right) \frac{\mathrm{e}^{A / 2} \mathrm{e}^{B / 2}}{\sqrt{\mathrm{e}^{A}-\mathrm{e}^{B}\left(\frac{\mathrm{d} x}{\mathrm{~d} t}\right)^{2}}} .
\end{aligned}
$$

Notemos que las funciones métricas presentes en las ecuaciones (4.31) y (4.32) deben estar evaluadas sobre la burbuja, esto es, en las coordenadas $(t, x(t))$. Estas ecuaciones deberán ser integradas junto con aquellas que determinan la evolución de la geometría externa.

Una restricción adicional se desprende del signo del radicando de la ecuación (4.31), que impone una cota inferior para la coordenada radial de la burbuja. Es decir, que $x$ debe ser tal que satisfaga

$$
\frac{1}{V^{2}}<R^{2}(t, x) .
$$

Durante toda la evolución supondremos válidas, además, las condiciones de energía débil [18] para la densidad de energía de la cáscara, es decir,

$$
\sigma>0 .
$$

Esta condición es equivalente a pedir que se satisfagan las siguientes restricciones

$$
\begin{aligned}
& \delta_{+}-\delta_{-}>\frac{\rho^{2} \sigma^{2}}{4}, \quad \text { si } \gamma_{+}=+1, \\
& \delta_{+}-\delta_{-}<\frac{\rho^{2} \sigma^{2}}{4}, \quad \text { si } \gamma_{+}=-1,
\end{aligned}
$$

con

$$
\delta=\frac{\dot{R}^{2}}{\mathrm{e}^{A}}-\frac{R^{\prime 2}}{\mathrm{e}^{B}} .
$$

Las condiciones (4.35) y (4.36) son generales y utilizadas para controlar el comportamiento de $\sigma(t)$ durante toda la evolución. La cantidad $\delta$ se calcula, según el caso, para las métricas de Lemaître, de LTB o de FLRW, a partir de las respectivas definiciones para las funciones $A(t, r)$ y $B(t, r)$. 


\subsubsection{Cálculo numérico de la evolución de la burbuja}

Para estudiar la evolución de la burbuja desarrollamos un código numérico en lenguaje Fortran 90. Las ecuaciones de evolución (4.31) y (4.32) para el radio y la densidad de la burbuja, respectivamente, quedan acopladas a aquellas que determinan la evolución de las funciones $R(t, r), M(t, r), \epsilon(t, r)$ y $B(t, r)$ que caracterizan la geometría externa. Estas últimas son obtenidas a partir de las expresiones (2.45), (2.46) y (2.48). El sistema completo de ecuaciones resulta $[150,151,248]$

$$
\begin{aligned}
\dot{R}(t, r) & =\mathrm{e}^{A(t, r) / 2}\left[\frac{2 M(t, r)}{R(t, r)}+\frac{\Lambda}{3} R^{2}(t, r)-1+R^{\prime 2}(t, r) \mathrm{e}^{-B(t, r)}\right]^{1 / 2}, \\
\dot{M}(t, r) & =-\frac{1}{2} R^{2}(t, r) \dot{R}(t, r) p(t, r) \\
\dot{\epsilon}(t, r) & =-p^{\prime}(t, r) \frac{\dot{R}(t, r)}{R^{\prime}(t, r)}-[\epsilon(t, r)+p(t, r)]\left[\frac{\dot{R}^{\prime}(t, r)}{R^{\prime}(t, r)}+2 \frac{\dot{R}(t, r)}{R(t, r)}\right], \\
\dot{B}(t, r) & =2\left[\frac{\dot{R}^{\prime}(t, r)}{R^{\prime}(t, r)}+\frac{\dot{R}(t, r) p^{\prime}(t, r)}{[\epsilon(t, r)+p(t, r)] R^{\prime}(t, r)}\right]
\end{aligned}
$$

con

$$
\begin{aligned}
& M^{\prime}(t, r)=\frac{1}{2} \epsilon(t, r) R(t, r) R^{\prime}(t, r), \\
& A(t, r)=-2 \int_{0}^{r} \frac{p^{\prime}(t, r)}{\epsilon(t, r)+p(t, r)} \mathrm{d} r,
\end{aligned}
$$

en donde los símbolos ${ }^{\prime} \mathrm{y}^{\prime}$ indican, respectivamente, derivadas con respecto a las variables $t$ y $r$. La presión $p(t, r)$, por su parte, se determina en cada instante a partir de la ecuación de estado correspondiente. La integración de las ecuaciones diferenciales parciales involucradas en la evolución de la geometría es realizada implementando el método de las líneas, con precisión de cuarto orden. ${ }^{5}$

\subsubsection{Condiciones iniciales para la evolución de la burbuja}

Las condiciones iniciales para resolver el problema quedan especificadas indicando la coordenada radial y la densidad de la burbuja en el instante inicial, $x_{0}$ y $\sigma_{0}$ respectivamente, y los perfiles iniciales de las funciones métricas $R\left(t_{0}, r\right), E(r)$ y $\epsilon\left(t_{0}, r\right) .{ }^{6}$ Comenzando por estos

\footnotetext{
${ }^{5} \mathrm{El}$ método de las líneas, utilizado para la resolución de ecuaciones diferenciales parciales, propone la discretización de la variable espacial $r$ en una grilla uniforme $r_{i}$ y un esquema de derivación numérica para reescribir las derivadas parciales con respecto a la variable $r$ en términos de las mismas funciones de evolución involucradas en la integración. El sistema de ecuaciones diferenciales ordinarias resultante puede ser entonces integrado, por ejemplo, mediante un algoritmo tipo Runge-Kutta [249].

${ }^{6}$ Notemos que estas tres funciones son suficientes para determinar completamente las condiciones iniciales. Las funciones $M\left(t_{0}, r\right)$ y $A\left(t_{0}, r\right)$ son luego calculadas a partir de las ecuaciones (4.42) y (4.43), respectivamente, y resulta $B\left(t_{0}, r\right)=\frac{R^{\prime}\left(t_{0}, r\right)}{1+2 E(r)}$.
} 
últimos, para nuestro problema particular elegimos:

$$
\begin{aligned}
& R\left(t_{0}, r\right)=a_{0} r \\
& E(r)=-\frac{1}{2}\left(\frac{r}{k}\right)^{2}, \\
& \epsilon\left(t_{0}, r\right)=\epsilon_{0}\left[1-\delta_{1} \exp \left(-\frac{\left(r-r_{0}\right)^{2}}{\delta_{2}^{2}}\right)\right],
\end{aligned}
$$

con $a_{0}, k$ y $\epsilon_{0}$ constantes arbitrarias que barren diferentes regiones del espacio de parámetros iniciales. Las cantidades $\delta_{1}, \delta_{2}$ y $r_{0}$ caracterizan la distribución inicial no homogénea de la densidad externa. ${ }^{7}$ Los escenarios de polvo o radiación homogéneos se recuperan con la elección $\delta_{1}=0$.

Los parámetros $\Lambda_{-}, \Lambda_{+}$y $\epsilon_{0}$ caracterizan las distintas geometrías. El parámetro $\Lambda_{-}$representa la energía de vacío de la región de universo emergente dentro de la burbuja, y está directamente asociado a la escala de energía que los modelos inflacionarios predicen para la etapa de nucleación [197]. Para una energía media de $\sim 10^{14} \mathrm{GeV}$, el valor de $\Lambda_{-}$en unidades de Planck resulta

$$
\Lambda_{-} \simeq 5 \times 10^{-5}
$$

En adelante, trabajaremos con dicho valor para caracterizar a la geometría interna. Los parámetros $\Lambda_{+}$y $\epsilon_{0}$ no poseen a priori restricciones provenientes de los modelos inflacionarios, puesto que los mismos suponen que, cualquiera sea la caracterización del fondo, la expansión inicial de la burbuja basta para dar lugar a un escenario con homogeneidad e isotropía. Dado que este es justamente el problema que estamos interesados en estudiar, elegiremos los parámetros que caracterizan el fondo de forma tal que la dinámica de diferentes escenarios pueda ser reproducida. En particular, trabajaremos con

$$
\epsilon_{0}=10 \Lambda_{-} \quad \text { y } \quad \Lambda_{+}<\epsilon_{0} .
$$

Dado que $\epsilon_{0}$ representa el valor asintótico de la densidad (lejos de la región no homogénea), la elección anterior asegura que, inicialmente, la dinámica de la región externa quede dominada por el término $(2 M / R)$ de la ecuación (4.38) y, en consecuencia, los eventuales efectos producidos por las distribuciones de polvo o radiación del fondo sean más pronunciados. ${ }^{8}$ Para el parámetro $\Lambda_{+}$elegiremos cuatro valores, correspondientes a diferentes evoluciones, a saber

$$
\Lambda_{+}=0, \quad \Lambda_{+}=\frac{1}{2} \Lambda_{-}, \quad \Lambda_{+}=\Lambda_{-}, \quad \Lambda_{+}=2 \Lambda_{-} .
$$

Resta especificar, por último, las condiciones iniciales para la cáscara delgada, elegidas como

$$
x_{0}=15 \quad \text { y } \quad \sigma_{0}=1 \times 10^{-3} .
$$

La elección de $x_{0}$ es tal que la nucleación se da en un punto del perfil no homogéneo en donde la cantidad $\rho^{\prime}$ es significativamente no nula. Pensando en el caso más general, en el cual la burbuja se expande en un fondo no homogéneo con presión no nula (es decir, descripto por la solución de Lemaître), esta elección se traduce en un gradiente de presión inicial no nulo que,

\footnotetext{
${ }^{7}$ Otra alternativa para introducir un perfil no homogéneo en el problema es a través de la función $E(r)$, como fue realizado en los trabajos [150, 234].

${ }^{8}$ Notemos que, de otra forma, la expansión diluiría rápidamente la densidad de materia de la región externa y el problema pasaría a ser un escenario tipo de Sitter-de Sitter.
} 
como fue discutido en la sección 2.2, da lugar a comportamientos peculiares en la dinámica del fondo y, eventualmente, también en la evolución de la burbuja (que es justamente lo que nos interesa estudiar). El valor inicial para $\sigma_{0}$ es elegido de forma tal que se satisfaga la condición (4.35). La ecuación de estado de la burbuja, $p_{\sigma}=w \sigma$, por su parte, queda caracterizada por los valores $w=0,1 / 3,-1 .^{9}$

Habiendo especificado completamente las cndiciones iniciales, presentamos a continuación los resultados de la evolución numérica de la burbuja de vacío inmersa en las diferentes geometrías de fondo. En orden creciente de complejidad, comenzaremos estudiando la evolución en un fondo homogéneo, con el fin de comparar los caso de polvo y radiación. Luego pasaremos a trabajar con los escenarios no homogéneos descriptos por las soluciones de LTB y de Lemaître. La discusión general sobre nuestros resultados está detallada al final del capítulo.

\subsection{Evolución en un fondo homogéneo: polvo vs. radiación}

Con el fin de estudiar los efectos que diferentes tipos de materia en la región externa tienen sobre la dinámica de la burbuja, comenzaremos analizando dos casos homogéneos, en los que la burbuja evoluciona o bien en un fondo con contenido de materia sin presión (esto es, polvo), o bien en un fondo con radiación. En ambos casos, polvo o radiación, la métrica que caracteriza el espacio externo es la de FLRW, con elemento de línea dado por

$$
\mathrm{d} s^{2}=\mathrm{d} t^{2}-a^{2}(t)\left(\frac{1}{1-k r^{2}} \mathrm{~d} r^{2}-r^{2} \mathrm{~d} \Omega^{2}\right),
$$

que se recupera de la expresión (4.11) cuando $E(r)=-\frac{1}{2} k r^{2}, R(t, r)=a(t) r$ y $\epsilon(t, r) \equiv \epsilon(t) .{ }^{10}$ Las ecuaciones (4.38)-(4.41), que determinan la evolución de la geometría, se reducen a

$$
\begin{aligned}
& \dot{a}^{2}=\frac{2 M}{a r^{3}}+a^{2} \frac{\Lambda_{+}}{3}-k, \\
& \dot{M}=-\frac{p}{2} \dot{a} r^{3}, \\
& \dot{\epsilon}=-3(\epsilon+p) \frac{\dot{a}}{a},
\end{aligned}
$$

con ecuaciones de estado $p=0$ y $p=\frac{1}{3} \epsilon$ para describir los contenidos de materia externa dados por polvo y radiación, respectivamente. Notemos que en el caso de un fondo homogéneo de polvo, resulta $\dot{M}(t, r) \equiv 0$.

Las ecuaciones (4.31) y (4.32) que determinan, respectivamente, la evolución de la coordenada radial y la densidad de la burbuja se reducen a la forma

$$
\begin{aligned}
\frac{\mathrm{d} x}{\mathrm{~d} t} & =\frac{-\left(1-k x^{2}\right) \dot{a} \pm \sqrt{\left(x^{2} a^{2} V^{2}-1\right)\left(1-k x^{2}\right)\left(a^{2} V^{2}-\dot{a}^{2}-k\right)}}{x\left(a^{2} V^{2}-k\right)} \\
\frac{\mathrm{d} \sigma}{\mathrm{d} t} & =-2\left(\sigma+p_{\sigma}\right) \frac{\dot{a}}{a}+\gamma_{+}(\epsilon+p)\left(\frac{\mathrm{d} x}{\mathrm{~d} t}\right) \frac{a}{\sqrt{1-k x^{2}}} \sqrt{1-\frac{a^{2}}{1-k x^{2}}\left(\frac{\mathrm{d} x}{\mathrm{~d} t}\right)^{2}},
\end{aligned}
$$

\footnotetext{
${ }^{9}$ Esta elección simple para la ecuación de estado representa una primera aproximación para abordar el problema. En una segunda instancia podría estudiarse la evolución de la burbuja de vacío utilizando ecuaciones de estado más realistas para describir el contenido de materia sobre la cáscara delgada, basadas en argumentos teóricos del modelo inflacionario particular que quiera estudiarse.

${ }^{10}$ En este límite, $p^{\prime}=0$, y de las ecuaciones (2.47) y (2.48) se tiene, respectivamente, $A(t, r)=0$ y $B(t, r)=$ $\frac{R^{\prime}(t, r)^{2}}{1+2 E(r)}=\frac{a(t)^{2}}{1-k r^{2}}$.
} 
con

$$
V^{2}=\Lambda_{-}+\left[\frac{\sigma}{4}+\frac{1}{\sigma}\left(\frac{\Lambda_{+}-\Lambda_{-}}{3}+\frac{2 M}{a^{3} x^{3}}\right)\right]^{2}
$$

\section{Resultados:}

En la figura 4.2 comparamos la evolución de la coordenada radial y la densidad de la burbuja en función del tiempo, para el caso de un fondo con radiación (líneas amarillas) y un fondo con polvo (líneas verdes). En ambos casos, los valores para el parámetro de ecuación de estado de la burbuja son $w=-1,0,1 / 3$ según las referencias indicadas en cada gráfico. Los parámetros que caracterizan la geometría, así como las condiciones iniciales para el cálculo de la evolución, son los detallados en la sección 4.2.2.

Del análisis de las curvas correspondientes a $x(t)$, podemos observar que el valor del parámetro $\Lambda_{+}$es determinante para la evolución de la burbuja. En los casos con $\Lambda_{+}<\Lambda_{-}$, la coordenada radial de la burbuja crece inicialmente (debido a la velocidad inicial con la que comienza la evolución, dada por la ecuación (4.54) evaluada en el instante $t=t_{0}$ ), para luego decrecer hasta alcanzar el límite inferior impuesto por la condición (4.33), esto es, $x>1 /(a V)$. Contrariamente, si $\Lambda_{-}<\Lambda_{+}$, la burbuja crece en forma indefinida. Este comportamiento es observado tanto para para los casos con polvo cuanto para los casos con radiación.

El comportamiento anterior puede entenderse en forma cualitativa si analizamos la expresión correspondiente a la aceleración de la burbuja con condición inicial $\dot{x}=0$. En este caso, tenemos [250]

$$
\left.\ddot{x}\right|_{\dot{x}\left(t=t_{0}\right)=0}=\left.\frac{1}{a}\left(\frac{\Lambda_{+}-\Lambda_{-}}{24 \pi \sigma}-2 \sigma \pi-\frac{2 \epsilon}{3 \sigma}\right)\right|_{t=t_{0}},
$$

y vemos que la nucleación de la burbuja con velocidad inicial nula presenta siempre aceleración negativa si $\Lambda_{+}<\Lambda_{-}$. De la ecuación (4.57) podemos inferir también que el contenido de materia en la región externa contribuye a disminuir la aceleración de la burbuja. Esto puede pensarse como una competencia de fuerzas entre la tensión superficial de la burbuja, que favorece el colapso, y la presión inducida por el calor latente de vacío, que favorece la expansión (en los casos con $0<\Lambda_{-}<\Lambda_{+}$). Si bien la ecuación (4.57) es válida sólo para condiciones iniciales muy particulares $(\dot{x}=0)$, este análisis cualitativo está en completo acuerdo con las curvas presentadas en la figura 4.2.

A pesar de que las curvas para el caso de un fondo con radiación tienen un comportamiento cualitativo similar al de aquellas para un fondo con polvo, podemos observar que la evolución es más lenta. Esto es, que en todos los casos estudiados, bajo las mismas condiciones iniciales, el fondo con radiación siempre retrasa el crecimiento del radio de la burbuja. Este comportamiento es consecuencia del efecto de la presión del fluido externo sobre la burbuja y, como discutiremos más adelante, puede tener relevancia para poner restricciones sobre los modelos inflacionarios.

Por otra parte, el contenido de materia en la burbuja modifica considerablemente la evolución. En todos los casos estudiados, el parámetro de ecuación de estado $w=0$ presenta una evolución más lenta que aquella calculada con $w=1 / 3$. Este comportamiento puede interpretarse también como una consecuencia de la presión ejercida por el fluido de radiación de la burbuja. Los casos con parámetro de ecuación de estado $w=-1$ alcanzan rápidamente la el límite impuesto por la condición (4.33), independientemente del tipo de fluido que caracteriza el fondo homogéneo. 

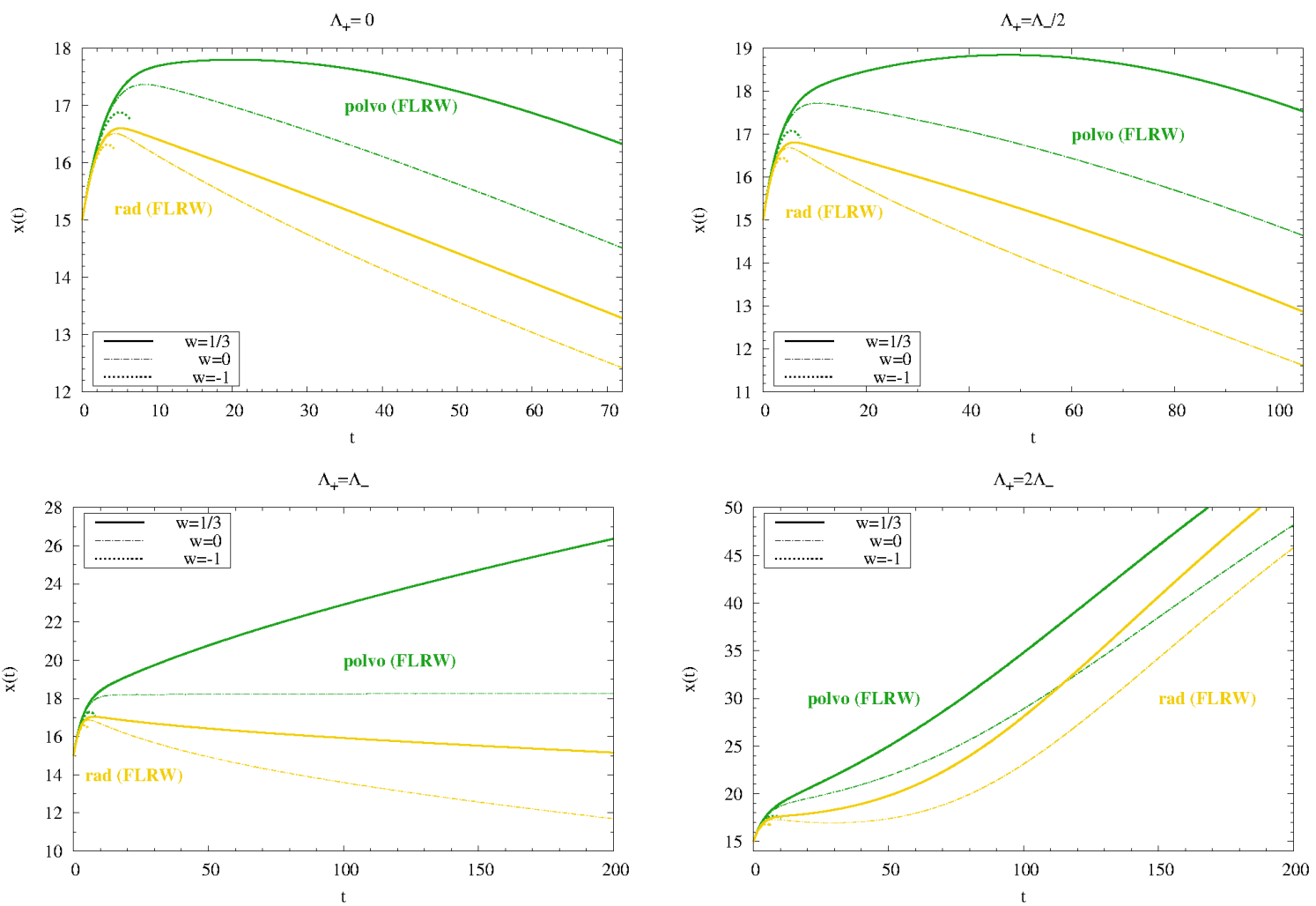

Figura 4.2: Evolución de la coordenada radial de la burbuja, en fondos homogéneos, para distintos valores del parámetro $\Lambda_{+}$. Las curvas verdes y amarillas indican, respectivamente, evoluciones en fondos con polvo o con radiación, para los parámetros de ecusación de estado $w=-1,0,1 / 3$.

En la figura 4.3 mostramos la evolución de la densidad de la burbuja para los dos valores extremos del parámetro $\Lambda$ (esto es, $\Lambda_{+}=0$ y $\Lambda_{+}=2 \Lambda_{-}$). Para ambas evoluciones, los casos con radiación mantienen una densidad menor en relación a aquellos con polvo, en acuerdo con la diferencia observada en la evolución de la coordenada radial $x(t)$. Sin embargo, para cada uno de los valores de $\Lambda_{+}$, no apreciamos diferencias cualitativas entre los diferentes $w$. Las evoluciones con $\Lambda_{+}=\Lambda_{-}$y $\Lambda_{+}=\Lambda_{-} / 2$ (omitidos en la figura 4.3) presentan comportamientos similares.

Una diferencia apreciable tiene lugar entre los casos con $\Lambda_{+}<\Lambda_{-}$y aquellos con $\Lambda_{-}<\Lambda_{+}$. Mientras que en los primeros el crecimiento de la coordenada radial de la burbuja alcanza un máximo y luego disminuye, las curvas para $x(t)$ en los segundos casos crecen en forma prácticamente monótona. Ampliaremos la discusión sobre este punto al final del capítulo. Por último, cabe mencionar que en el caso con $\Lambda_{+}>\Lambda_{-}$, para tiempos grandes en los que la densidad de materia del fondo se diluye debido a la expansión, la evolución tiende al límite del caso con un fondo descripto por la métrica de de Sitter (cuya forma analítica es conocida, y fue estudiada numéricamente en [234]), lo cual constituye un test para el código numérico desarrollado. 

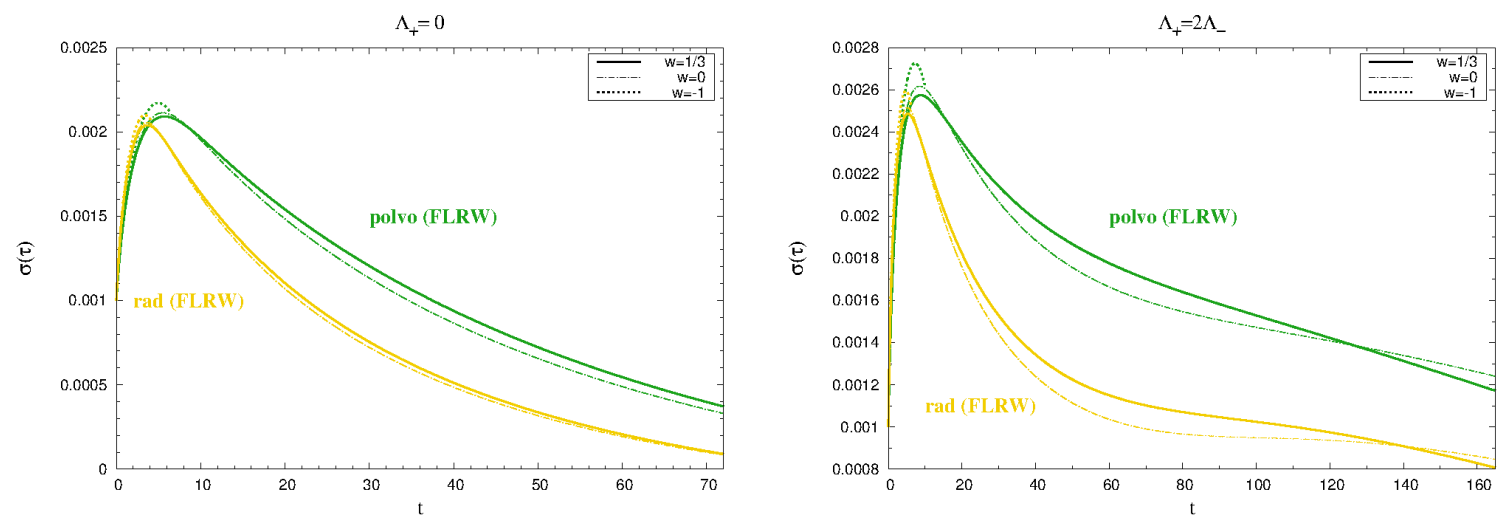

Figura 4.3: Evolución de la densidad de la burbuja para los valores extremos del parámetro $\Lambda\left(\Lambda_{+}=0\right.$ y $\left.\Lambda_{+}=2 \Lambda_{-}\right)$. Los casos con radiación mantienen una densidad menor en relación a aquellos con polvo. No apreciamos diferencias cualitativas para los diferentes $w$. Las evoluciones con $\Lambda_{+}=\Lambda_{-}$y $\Lambda_{+}=\Lambda_{-} / 2$ (omitidos en esta figura) presentan comportamientos similares.

\subsection{Evolución en fondos no homogéneos}

Con el propósito de analizar los posibles efectos que una distribución no homogénea de materia en la región externa puede tener sobre la dinámica de la burbuja, presentaremos en esta sección la evolución en fondos descriptos por las soluciones de LTB y de Lemaitre. Mientras que el primer caso fue analizado en otros trabajos [234, 235, 238], la evolución de burbujas en ambientes no homogéneos de radiación, descriptos por la solución de Lemaître, no ha sido previamente estudiada y constituye el principal aporte de este trabajo. Los resultados de dichaa evolución serán comparados con la evolución en un fondo homogéneo de radiación, así como también con los casos no homogéneos de polvo.

\subsubsection{Evolución en un fondo no homogéneo de polvo}

Para describir la evolución de una burbuja inmersa en un fondo con distribución no homogénea de polvo, caracterizamos la región externa con la solución de LTB. El elemento de línea es

$$
\mathrm{d} s^{2}=\mathrm{d} t^{2}-\frac{R^{\prime 2}(t, r)}{1+2 E(r)} \mathrm{d} r^{2}-R^{2}(t, r) \mathrm{d} \Omega^{2},
$$

y puede ser obtenido, nuevamente, de la expresión (4.11) para el caso $p(t, r)=0$ (y, en consecuencia, $\left.p^{\prime}(t, r)=0\right)$. Las ecuaciones que determinan la evolución de la geometría externa son

$$
\begin{aligned}
\dot{R}^{2} & =\frac{2 M}{R}+\frac{\Lambda}{3} R^{2}+2 E(r), \\
\dot{\epsilon} & =-\epsilon\left(\frac{\dot{R}^{\prime}}{R^{\prime}}+2 \frac{\dot{R}}{R}\right),
\end{aligned}
$$

en donde $M(t, r) \equiv M(r)$. Las ecuaciones que determinan la evolución de la coordenada radial y la densidad de la burbuja resultan 


$$
\begin{aligned}
\frac{\mathrm{d} x}{\mathrm{~d} t} & =\frac{-(1+2 E) \dot{R} \pm \sqrt{\left(R^{2} V^{2}-1\right)(1+2 E)\left(2 E-\dot{R}^{2}+R^{2} V^{2}\right)}}{R^{\prime}\left(2 E+R^{2} V^{2}\right)} \\
\frac{\mathrm{d} \sigma}{\mathrm{d} t} & =-2\left(\sigma+p_{\sigma}\right) \frac{\dot{R}}{R}+\gamma(\epsilon+p)\left(\frac{\mathrm{d} x}{\mathrm{~d} t}\right) \frac{R^{\prime} / \sqrt{1+2 E}}{\sqrt{1-\frac{R^{\prime 2}}{(1+2 E)}\left(\frac{\mathrm{d} x}{\mathrm{~d} t}\right)^{2}}}
\end{aligned}
$$

con

$$
V^{2}=\Lambda_{-}+\left[\frac{\sigma}{4}+\frac{1}{\sigma}\left(\frac{\Lambda_{+}-\Lambda_{-}}{3}+\frac{2 M}{R^{3}}\right)\right]^{2}
$$

\section{Resultados:}

En la figura 4.4 mostramos la evolución de la coordenada radial de la burbuja en un fondo no homogéneo de polvo (curvas rojas), para los diferentes valores del parámetro $\Lambda_{+}$y los parámetros de ecuación de estado $w=-1,0, \frac{1}{3}$. Los gráficos de la derecha muestran, en cada caso, la evolución del perfil radial de densidad del fondo no homogéneo. Debido a la expansión, la región no homogénea inicial se diluye al transcurrir el tiempo. Es decir, que asintóticamente la evolución tiende a la de un fondo homogéneo. Las cruces negras representan, a cada tiempo, la coordenada radial de la burbuja.

Con el propósito de analizar cuáles son las consecuencias de que la nucleación de la burbuja tenga lugar en un fondo no homogéneo, incluimos los perfiles correspondientes a evoluciones con iguales condiciones iniciales, pero en un fondo de FLRW con contenido polvo (curvas verdes). La densidad del fondo homogéneo corresponde al valor asintótico del perfil de densidad no homogéneo. De la comparación de los casos homogéneo y no homogéneo, puede apreciarse que la evolución de $x(t)$ es cualitativamente indistinguible. Sin embargo, en el fondo no homogéneo las curvas se muestran trasladadas verticalmente (sin alterarse, sin embargo, la forma general de la curva).

Para entender el comportamiento anterior, basta tener en cuenta que la condición inicial elegida para $x_{0}$ (es decir, la coordenada radial en donde se produciría la nucleación de la burbuja) es tal que la evolución comienza en un punto de subdensidad en relación al valor asintótico del fondo. Si tenemos en cuenta que la velocidad inicial de la burbuja es directamente proporcional a $\epsilon$ (a través de $V^{2}$ en a la ecuación (4.61)), es entonces esperable que la evolución comience con una velocidad menor. Sin embargo, la evolución en tiempos subsiguientes no presenta cambios cualitativos respecto del caso homogéneo. Con esto, podemos inferir que la distribución no homogénea tiene influencia en la evolución sólo a través del valor de la densidad inicial. Una vez iniciada la evolución, el comportamiento de la burbuja puede describirse con el escenario de un fondo homogéneo con densidad de fondo igual a la densidad inicial en el punto de nucleación. Es importante destacar que cuantitativamente las evoluciones entre los casos homogéneo y no homogéneo son diferentes. Si la nucleación de la burbuja tiene lugar en una región de subdensidad lo suficientemente baja, la evolución podría presentar incompatibilidades con los requisitos impuestos por las condiciones del escenario inflacionario que se quiera estudiar. Ampliaremos esta discusión hacia el final de este capítulo.

El caso $\Lambda_{+}=2 \Lambda_{-}$es cualitativamente diferente a los otros, y no sigue el análisis anterior. En este caso, es la diferencia $\left(\Lambda_{+}-\Lambda_{-}\right)$la que domina el comportamiento de la función auxiliar $V(t)$, y densidades iniciales menores parecen contribuir a una expansión más rápida. 
Por su parte, las diferencias observadas entre las evoluciones correspondientes a diferentes ecuaciones de estado para el contenido de materia sobre la burbuja son similares a aquellas encontradas en los casos homogéneos de polvo y radiación (esto es, que el valor $w=1 / 3$ favorece la evolución).

\subsubsection{Evolución en un fondo no homogéneo de radiación}

Hemos estudiado hasta aquí los efectos que un contenido de materia con presión puede tener sobre la evolución de la burbuja (sección 4.3), así como también los diferencias apreciables en aquellos casos en los que la burbuja se nuclea en una región de subdensidad (sección 4.4.1). En vista de los resultados obtenidos en dichos análisis, y dado que es esperable la presencia de materia ultrarelativista en las etapas más tempranas de la evolución del universo, es relevante investigar cómo es la evolución de la burbuja en un fondo de radiación con distribución no homogénea (es decir, descripto por la solución de Lemaître). Este caso, por su parte, cobra especial interés debido al tipo de evolución no local de la región no homogénea.

La evolución de la geometría descripta por la métrica de Lemaître obedece las ecuaciones generales (4.31) y (4.32) presentadas anteriormente. Las figuras 4.5 y 4.6 muestran la evolución de la burbuja comparada, respectivamente, con los casos de un fondo homogéneo de radiación y un fondo no homogéneo de polvo.

Al igual que para la solución de LTB, la evolución de los perfiles de densidad está presentada en los gráficos de la derecha de la figura 4.5. A diferencia de la primera, y de acuerdo a la evolución propia de la geometría detallada en la sección 2.2 , la región no homogénea no se mantiene confinada a su rango inicial para la coordenada radial. Debido al efecto de un gradiente de presión no nulo, las regiones no homogéneas se dislocan a lo largo de la evolución hacia valores de $r$ mayores o menores, según el signo de $p^{\prime}$.

\section{Resultados:}

El análisis de la figura 4.5, en donde comparamos la evolución de la burbuja en fondos de radiación (homogéneo y no homogéneo), muestra características similares a las encontradas en la comparación de los fondos de polvo (figura 4.4). La evolución en el fondo descripto por la solución de Lemaître es cualitativamente igual al caso de un fondo homogéneo con radiación. Esta característica es esperable, dado que la expresión de la función $V^{2}$ no involucra a la presión del fluido. Algunas diferencias cualitativas son apreciables en los casos con $\Lambda_{-} \leq \Lambda_{+}$, probablemente debidas al pasaje de la burbuja por la región no homogénea (indicado con las cruces negras en los gráficos de la derecha). La evolución, sin embargo, no se modifica cualitativamente.

Las diferencias observadas para los distintos contenidos de materia sobre la burbuja son similares a las de los casos anteriores y, de la misma forma, las burbujas con parámetro de ecuación de estado $w=-1$ alcanzan rápidamente la cota inferior impuesta por la condición (4.33).

La comparación de las evoluciones en fondos no homogéneos de polvo y radiación (figura 4.6) muestra diferencias cuantitativas, pero no cualitativas, análogas a aquellas observadas en la comparación de los dos homogéneos (figura 4.2). En todos los casos la evolución de la burbuja en el fondo de radiación es más lenta, debido a la resistencia ejercida por la presión del fluido externo. 

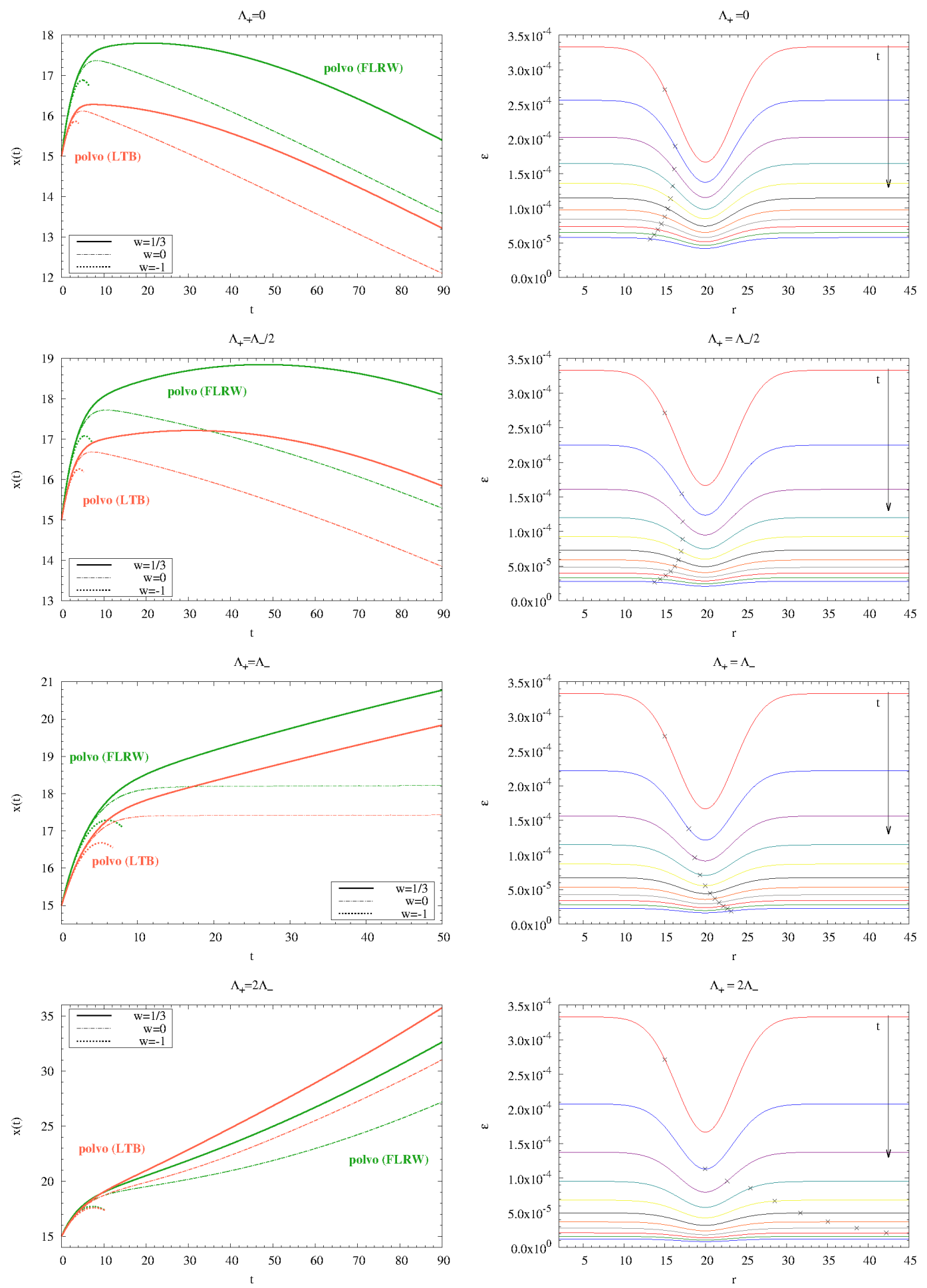

Figura 4.4: Izquierda: Evolución de la coordenada radial de la burbuja en un fondo de polvo no homogéneo descripto por la solución de LTB (curvas rojas). Las curvas verdes representan la evolución de un fondo homogéneo con densidad igual al valor asintótico del perfil no homogéneo. Derecha: Evolución del perfil de densidad del fondo no homogéneo de polvo. Las cruces negras representan la coordenada radial de la burbuja para cada tiempo. 

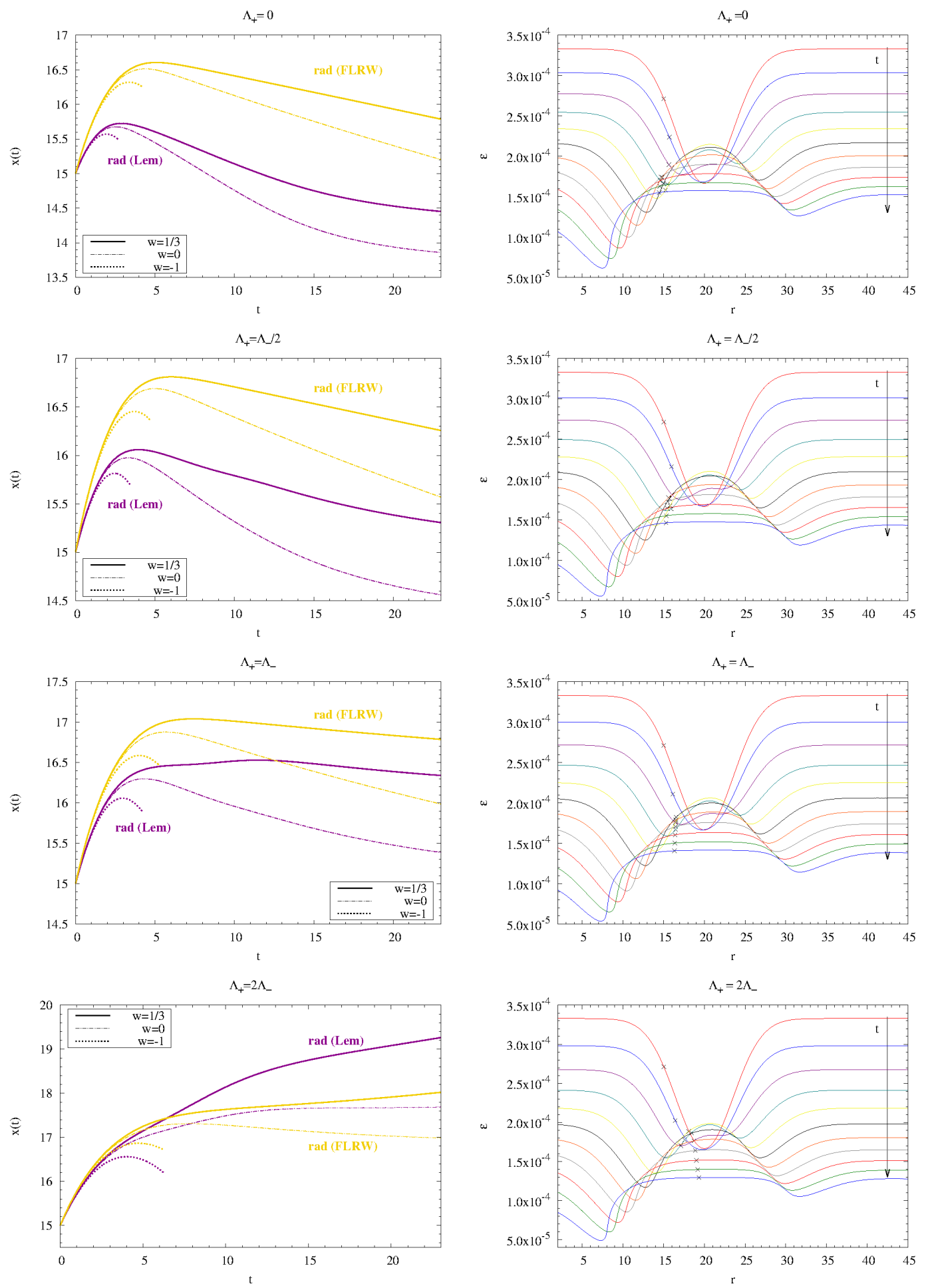

Figura 4.5: Izquierda: Evolución de la coordenada radial de la burbuja en un fondo de radiación no homogéneo descripto por la solución de Lemaître (curvas violetas). Las curvas amarillas representan la evolución de un fondo homogéneo de radiación con densidad igual al valor asintótico del perfil no homogéneo. Derecha: Evolución del perfil de densidad del fondo no homogéneo de radiación. Las cruces negras representan la coordenada radial de la burbuja en cada tiempo. 

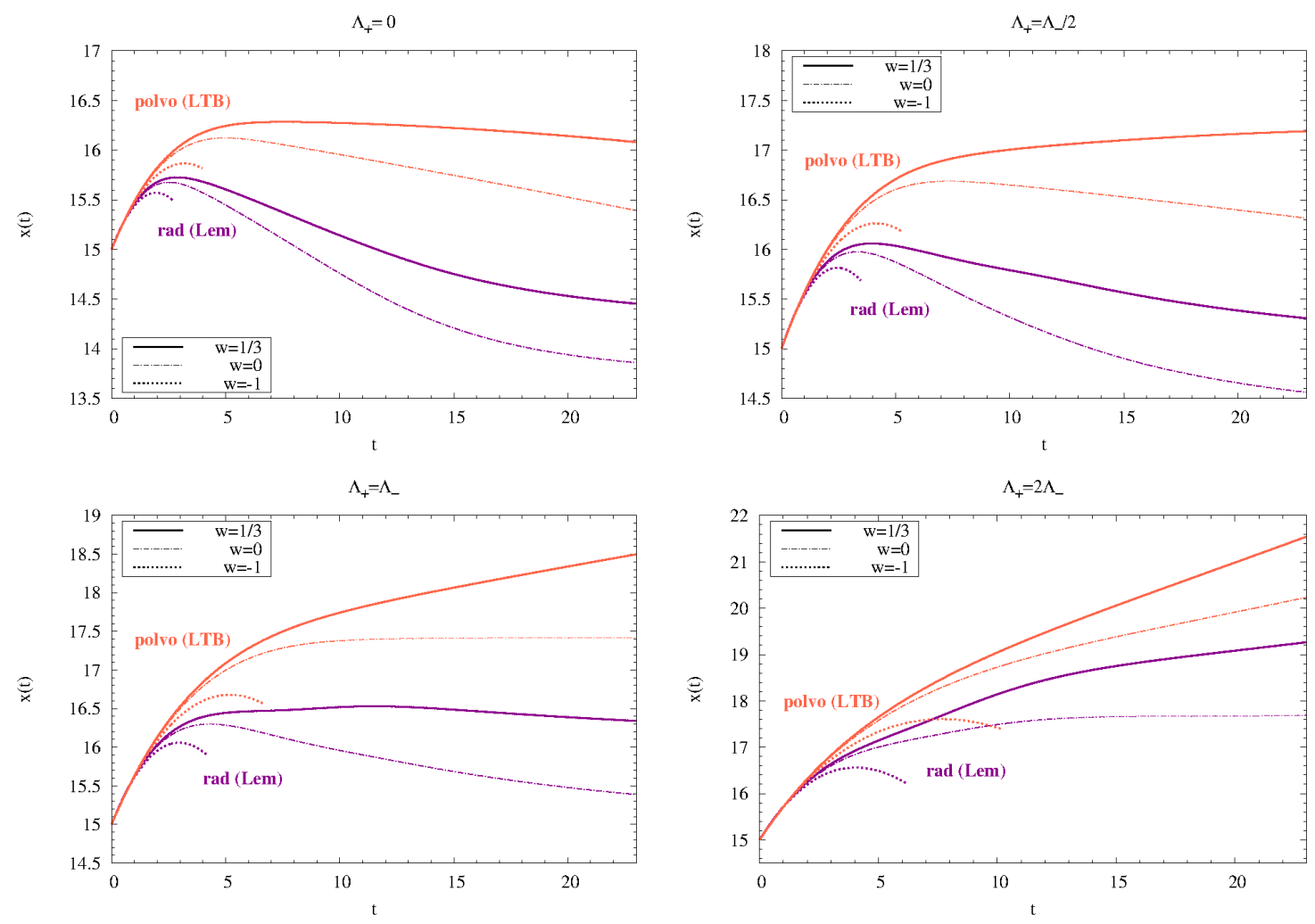

Figura 4.6: Comparación de las evoluciones en fondos no homogéneos de polvo (curvas rojas) y radiación (curvas violetas). La geometría del fondo está descripta, en cada caso, por las soluciones de LTB y de Lemaître, respectivamente. 

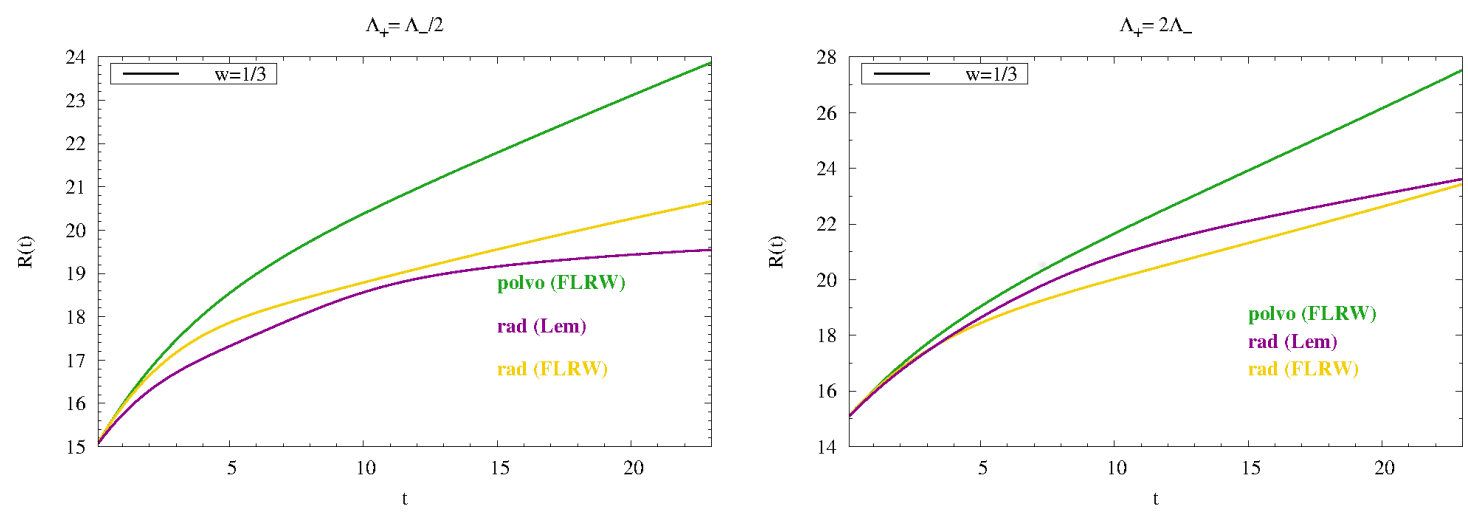

Figura 4.7: Evolución del radio propio de la burbuja, $R(t, x(t)=\rho(\tau)$, para los casos homogéneos y para el fondo no homogéneo de radiación. El creciento es sensible al contenido de materia del ambiente, siendo más lento si la burbuja evoluciona en un entorno con radiación.

\subsection{Discusión general}

Con la motivación de estudiar escenarios generales para el proceso de inflación cosmológica, hemos presentado en este capítulo la evolución de burbujas de vacío en fondos de materia y radiación, con distribuciones homogénea y no homogénea, en grado creciente de complejidad. Los casos con radiación (homogénea o no) no han sido estudiados previamente y constituyen el principal aporte original de este trabajo.

La evolución de la burbuja de vacío fue calculada numéricamente a partir del formalismo de cáscara delgada o thin shell. La resolución general del problema envuelve la integración temporal de la coordenada radial de la burbuja y su densidad, junto con la evolución de la geometría del fondo. Esta última, para los casos no homogéneos descriptos por la solución de LTB y de Lemaître involucra la integración de ecuaciones diferencias parciales no lineales. El código numérico que desarrollamos reproduce perfectamente los resultados obtenidos previamente para los casos de una evolución en un fondo de polvo [234, 235], y generaliza el estudio para casos con radiación (en particular, con distribución no homogénea).

Los resultados que obtuvimos son múltiples. Comenzando por la comparación de la evolución en fondos homogéneos con contenido de polvo y radiación, mostramos que la presión de la radiación del fondo externo ejerce un efecto de retraso en el crecimiento de la burbuja (figura 4.2).

La evolución para los casos con fondos no homogéneos descriptos por la solución de LTB y de Lemaître fueron comparados, respectivamente, con la evolución homogénea de un fondo de polvo y radiación. Los resultados obtenidos muestran que si la burbuja de vacío se nuclea en una región de subdensidad, la evolución es equivalente a la de un fondo homogéneo, con densidad inicial igual a la densidad del punto en donde ocurre la nucleación. Es decir, que la distribución no homogénea del fondo sólo tiene una influencia apreciable sobre las condiciones iniciales. Este comportamiento se repite tanto en los casos con contenido de polvo, como aquellos con radiación.

En relación a las diferencias observadas para distintos tipos de ecuación de estado para caracterizar el contenido de materia sobre la burbuja, encontramos que los parámetros de ecuación de estado $w=0$ y $w=1 / 3$ dan lugar a evoluciones similares. Sin embargo, una 
burbuja de radiación se expandería más rápido que una de polvo, como consecuencia de la presión del material de la propia burbuja sobre el fondo. En todos los casos estudiados, la evolución correspondiente al parámetro de ecuación de estado $w=-1$ alcanza rápidamente el límite impuesto por la ecuación (4.33) sobre la coordenada radial de la burbuja.

Algunas diferencias significativas pueden apreciarse también para las distintas elecciones del parámetro $\Lambda_{+}$. En los casos con $\Lambda_{+}<\Lambda_{-}$, el crecimiento del radio de la burbuja se frena y da lugar a una disminución de la coordenada radial de la burbuja, mientras que para $\Lambda_{-}<\Lambda_{+}$el crecimiento de $x(t)$ es prácticamente monótono. El primer caso, sin embargo, no debe interpretarse erróneamente como un colapso de la burbuja. Dado que $x(t)$ representa la coordenada radial de la burbuja, no comóvil con el fondo, el crecimiento o decrecimiento de esta coordenada indica que la burbuja de vacío se expande más o menos rápido se la propia expansión del fondo en el que esta está inmersa.

Para concluir, cabe resaltar que si bien no detectamos diferencias cualitativas en las distintas evoluciones, las diferencias cuantitativas que observamos entre los casos de polvo y radiación (debidas a la presión de la radiación sobre la burbuja), podrían tener efectos relevantes sobre el desarrollo de la inflación. Para apreciar esto, mostramos en la figura 4.7 la evolución del radio propio de la burbuja, $R(t, x(t))=\rho(\tau)$. Dado que dicho radio actúa como una frontera móvil de la región inflacionaria, las consecuencias de su evolución sobre los campos definidos en la región interior deben ser tenidos en cuenta en un análisis minuscioso de los modelos inflacionarios. 
"Solamente el farolero del único farol del Polo Norte y su colega del único farol del Polo Sur llevaban una vida ociosa e indiferente: trabajaban dos veces al año."

El Principito (1943)

Antoine de Saint-Exupéry 


\section{Capítulo 5}

\section{Modelos de void con dos fluidos}

Las llamadas regiones de vacío o voids son subdensidades pronunciadas en la distribución de materia, caracterizadas perfiles radiales no homogéneos. Los voids con simetría esférica pueden ser modelados con las soluciones de Lemaître y de LTB para describir, respectivamente, fluidos con y sin presión.

Los modelos cosmológicos no homogéneos construidos a partir la solución de LTB con perfiles de void (en adelante, modelos de void de LTB), han sido ampliamente estudiados con el objeto de describir diferentes conjuntos de observaciones astronómicas sin la necesidad de introducir una componente de energía oscura en el universo. Mientras que algunos autores han desestimado la viabilidad de este tipo de modelos para describir simultáneamente conjuntos de datos de diferente naturaleza (e.g. observaciones del CMB, SN tipo Ia, BAO y $H_{0}$, entre otras), trabajos recientes sugieren que los modelos de void de LTB pueden adquirir relevancia si incluimos los efectos de una distribución no homogénea de radiación en la evolución temprana del universo.

En este contexto, un modelo cosmológico de dos fluidos no comóviles (esto es, materia y radiación) fue presentado recientemente por C. Clarkson, M. Regis y W.C. Lim [140]. El mismo contempla aquellas correcciones en las geodésicas nulas que surgen como consecuencia de la evolución no trivial de la geometría de fondo. La dinámica completa del modelo es calculada en forma numérica integrando las ecuaciones de evolución para los dos fluidos, junto con aquellas que caracterizan la geometría. Este modelo es una primera versión del problema, en la que algunas hipótesis razonables son consideradas. En particular, los efectos de la presión anisótropa de la radiación (no nula para el caso de distribuciones no homogéneas), son omitidas durante el cómputo de la evolución.

Presentaremos en este capítulo un trabajo realizado en colaboración con los autores mencionados anteriormente, que propone una generalización del modelo de dos fluidos para el caso en el que el cuadrupolo de la distribución no homogénea de la radiación (esto es, la presión no isótropa) es tenido en cuenta. Comenzaremos por introducir los modelos de void y detallar los argumentos que justifican la relevancia de incluir los efectos de una distribución no homogénea de la radiación en la evolución de la geometría. Luego describiremos el modelo de dos fluidos no comóviles, así como la evolución de los multipolos de orden superior de la distribución de radiación no homogénea a partir del formalismo de la teoría cinética relativista. Los potenciales efectos de la presión anisótropa de la radiación en cantidades físicas observables que permitan distinguir entre diferentes modelos cosmológicos serán discutidos al final del capítulo. 


\subsection{Los modelos de void}

Los modelos de void han sido propuestos como un alternativa viable a la introducción de una constante cosmológica (o de campos de materia de naturaleza desconocida, como aquel que describiría a la energía oscura) en la descripción del universo [133]. Estos modelos están basados usualmente en la solución de LTB, y son caracterizados por una distribución no homogénea de polvo con una región pronunciada de subdensidad en el centro de simetría de la métrica. Lejos de dicha región, sin embargo, la distribución de materia adopta un comportamiento homogéneo. Un perfil típico utilizado para caracterizar a la región de subdensidad (o void, como suele denominarse comúnmente en la literatura) está mostrado en la figura 5.1 $[251] .^{1}$

Las soluciones de LTB que describen voids esféricos tienen el potencial de modelar los efectos locales que puedan tener lugar en la región de subdensidad, con la ventaja de recuperar asintóticamente el comportamiento de la solución de FLRW en el límite homogéneo, esto es, lejos del centro de simetría. En este sentido, han sido utilizados para mostrar que es posible ajustar aquellas observaciones que indican aceleración en la expansión del universo sin postular la existencia de energía oscura. Constituyen así una primera aproximación al problema general con menos simetrías. ${ }^{2}$

Dadas las limitaciones observacionales para discernir entre las evoluciones temporal y radial de la geometría, y la falta de un mecanismo eficiente para describir la formación de voids, la forma más adecuada de trabajar con este tipo de distribuciones es a partir de una reconstrucción directa del perfil (en lugar de poner cotas sobre un modelo paramétrico simple). Para ello, debemos considerar condiciones físicas independientes en el centro y en la región asintótica, en acuerdo con los diferentes tipos de observaciones astronómicas que pretendemos ajustar con el modelo.

Los conjuntos de datos que permiten reconstruir un modelo de void son de naturaleza variada [133]. Si consideramos un observador en el centro de simetría, las observaciones locales (como la curva de la distancia de luminosidad vs. redshift de las SN tipo Ia) permiten poner cotas sobre la curvatura, la tasa de expansión de Hubble y la densidad de materia en el centro, así como también sobre los perfiles radiales no homogéneos de estas cantidades físicas hasta distancias correspondientes a algunas unidades de redshift. Por otra parte, las observaciones del fondo cósmico de radiación ponen cotas sobre la región externa de los perfiles de void, a partir de la relación $d_{A}(z)$ para distancias correspondientes a $z \sim 1100$, de la fracción de bariones, $f_{b}$, y del cociente entre el número de bariones y el número de fotones, $n_{b} / n_{\gamma}$, a una distancia comóvil aproximada de 13 Gpc medida desde el centro de simetría. Los grados de libertad restantes, necesarios para especificar la variación radial de la fracción de bariones, de la densidad de la energía de radiación y del espectro primordial de potencias, deben ser, sin embargo, restringidos a partir de otros conjuntos de observaciones [133].

\footnotetext{
${ }^{1}$ En adelante, trabajaremos con modelos de void que describen subdensidades a tiempos tardíos en la escala de los Gpc. Notemos, sin embargo, que existen otros modelos (también llamados de 'void'), que representan regiones no homogéneas del orden de algunos cientos de Mpc. Estos últimos son estudiados, usualmente, como un tratamiento perturbativo del modelo de FLRW [133]. Es importante mencionar que no disponemos aún de mecanismos eficientes que den lugar a la formación de tales voids y, en esta dirección, probablemente sea necesario proponer algún proceso desconocido en la etapa final de la inflación que justifique el surgimiento de una región con simetría esférica a escalas de Hubble [133].

${ }^{2}$ Estas características imitan de manera más realista aquellos primeros modelos no homogéneos propuestos por Tomita, basados en el pegado de dos soluciones homogéneas con diferente parámetro de expansión (sección 1.3).
} 


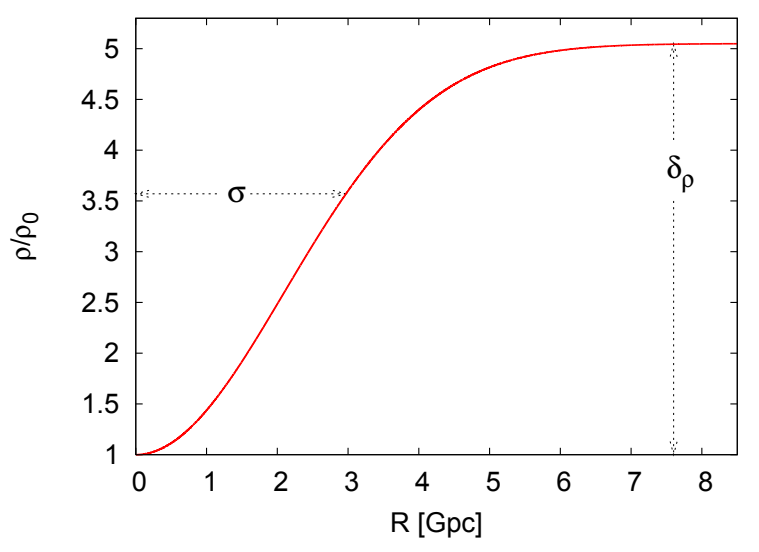

Figura 5.1: (extraída de [251]) Ejemplo de un perfil de densidad de materia característico de los modelos de void, para un dado instante $t_{0}$. La distribución no homogénea está especificada, en este caso particular, por la función $\rho\left(t_{0}, r\right)=\rho_{0}\left[1+\delta_{\rho}-\delta_{\rho} \exp \left(-\frac{r^{2}}{\sigma^{2}}\right)\right]$, con $\rho_{0}=0,3 \times$ $\left(3 H_{0}^{2}\right) /(8 \pi G), \delta_{\rho}=4,05$ y $\sigma=2,96$.

En esta dirección, varios trabajos se han desarrollado utilizando los datos del fondo cósmico de radiación para poner restricciones sobre los modelos de void [129, 174, 251, 252]. Los resultados de los mismos indican que efectivamente es posible ajustar un dado modelo a partir de las observaciones provenientes del fondo cósmico de radiación. Sin embargo, las restricciones impuestas por el CMB no serían compatibles con valores razonables del parámetro $H_{0}$, ni con el ajuste simultáneo de otros conjuntos de datos, sugiriendo entonces que los modelos de void no serían viables para describir adecuadamente el universo observable [253, 254, 255, 256].

En todos estos trabajos, la influencia de la radiación no fue considerada para modelar la evolución a tiempos tempranos. Esta simplificación parece ser razonable porque la radiación contribuye apenas con un $10 \%$ al contenido total de densidad de energía en el momento de último scattering, con lo cual es esperable que el error cometido al calcular los parámetros de los modelos de void sin radiación pueda ser despreciado.

Este escenario fue reconsiderado recientemente por C. Clarkson y M. Regis [133]. Los autores de este trabajo analizaron detalladamente las correcciones que sufren los fotones provenientes del fondo cósmico de radiación en su trayectoria desde la superficie de último scattering. Dichas correcciones pueden clasificarse en dos tipos:

1. correcciones geométricas, introducidas a través de la dinámica intrínseca del fondo, que afectan directamente el cálculo de la distancia $d_{A}$ a la superficie de último scattering (similares a aquellas introducidas por modelos simples de energía oscura); y

2. correcciones debidas al efecto Sachs-Wolfe integrado (SWI), que depende de la evolución de las perturbaciones primordiales. ${ }^{3}$

Las correcciones mencionadas en el primer grupo fueron estudiadas en [133]. Los resultados muestran que ciertos modelos de void pueden ajustar el espectro de potencias del fondo

\footnotetext{
[257].

${ }^{3}$ La generalización de este tipo de correcciones para el caso de los modelos de LTB puede encontrarse en
} 
cósmico de radiación, en buen acuerdo con los datos provenientes de SN tipo Ia y con estimaciones del parámetro de expansión de Hubble local, si la influencia de una distribución no homogénea de la radiación es tenida en cuenta en la evolución de la geometría. Estos resultados fueron acompañados de argumentos teóricos sólidos y estimaciones semi-analíticas que justifican fuertemente la necesidad de incluir los efectos de un perfil no homogéneo de radiación sobre la evolución de la geometría durante las etapas tempranas del universo.

\subsubsection{La importancia de la radiación en los modelos de void}

Como mencionamos anteriormente, una característica común en los trabajos que desestiman la construcción de modelos cosmológicos viables a partir de perfiles de void es la de despreciar los efectos de la radiación en la evolución temprana del universo. Esta simplificación parece en principio razonable si tenemos en cuenta que la radiación contribuye apenas un $10 \%$ a la densidad de energía total en el momento de último scattering, y que la relación $d_{A}(z)$ en los modelos de FLRW varía en un porcentaje casi despreciable para $z \sim 1100$ si la radiación es tenida en cuenta [133]. Es esperable, entonces, que los errores inducidos por esta simplificación no sean significativos en la subsecuente estimación de los parámetros del modelo.

Sin embargo, lejos de actuar como una componente secundaria, la inclusión de la radiación en la evolución temprana de la geometría puede tener efectos no despreciables en los modelos de void [150]. Como fue sugerido primero en [28], y argumentado más tarde en [133], las cotas impuestas por el CMB sobre un modelo cosmológico completo, que lleve en cuenta los efectos de la radiación en la dinámica del espacio-tiempo, son considerablemente menos restrictivas. La influencia de la radiación en la evolución temprana del universo puede ser significativa si tenemos en cuenta los siguientes aspectos: [133]

1. La inclusión de un perfil no homogéneo para la radiación incorpora al modelo un grado de libertad adicional, usualmente restringido en forma artificial en los modelos de LTB con contenido de polvo únicamente.

2. La presencia de la radiación en tiempos tempranos se imprime intrínsecamente en la dinámica de tiempos tardíos a través de la propia evolución de la geometría.

3. Las observaciones del fondo cósmico de radiación no determinan, por si mismas, el interior de nuestro cono de luz pasado (importante para estudiar otros aspectos del modelo que puedan cotejarse con observaciones independientes).

Para profundizar los argumentos arriba mencionados, consideremos el siguiente análisis cualitativo. Sean $T_{*}^{\text {(asintótica) }}$ y $T_{0}^{\text {(asintótica) }}$ las temperaturas de los fotones del CMB, medidas ambas en la región asintótica exterior al void, en dos instantes $t_{*}$ y $t_{0}$, que representan, respectivamente, el tiempo de desacople de los fotones en la superficie de último scattering y un tiempo posterior en la evolución tardía del universo (digamos, hoy). El factor de escala en el momento del desacople viene dado por la expresión

$$
a_{*}^{\text {(asintótica) }}=\frac{T_{0}^{\text {(asintótica) }}}{T_{*}^{(\text {asintótica })}} .
$$

Si las temperaturas $T_{0}^{(\text {centro })}$ y $T_{*}^{(\text {centro })}$ difieren, la relación anterior puede ser significativamente diferente de aquella utilizada para calcular el redshift correspondiente a la superficie 
de último scattering medido desde el centro del void, que escribimos como

$$
\frac{1}{\left(1+z_{*}\right)}=\frac{T_{0}^{\text {(centro) }}}{T_{*}^{(\text {asintótica) }}} .
$$

Como consecuencia, la relación entre el factor de escala y el redshift (utilizada en modelos de FLRW) puede verse modificada considerablemente en los modelos de void si los efectos de la radiación sobre la dinámica son tenidos en cuenta [133].

Para analizar esto, consideremos un modelo de LTB puro (sin radiación). Como vimos, la determinación de $a(z)$ en este caso difiere de aquella utilizada en los modelos de FLRW, $a(z)=1 /(1+z)$. Esta diferencia representa un porcentaje típico de algunas pocas unidades de redshift para $z \sim 1100$, y hace que la temperatura del CMB hoy (sobre una hipersuperficie de $t=$ cte) varíe en un porcentaje también pequeño a lo largo de un perfil de void monótono. Como consecuencia, la variación en la densidad de radiación queda también restringida a un nivel menor (recordemos que $T \propto \Omega_{\gamma}^{1 / 4}$ ). Esta condición es, en efecto, la que impone límites directos sobre la diferencia del valor del parámetro de expansión de Hubble para tiempos tardíos entre el centro del void y la región asintótica. Sin embargo, en los modelos de void es esperable que esta diferencia sea considerablemente mayor (para estar justamente en buen acuerdo con otras cotas observacionales, como aquellas provenientes de las curvas de la distancia de luminosidad de las SN tipo Ia). Es decir, que es esperable que la tasa de expansión en el centro del void sea mayor que la región exterior y, en consecuencia, que la temperatura del CMB en el centro sea significativamente menor. Esta inconsistencia justifica entonces la necesidad de incluir un perfil no homogéneo de radiación para una correcta formulación del modelo [133]. ${ }^{4}$

Para abordar de forma adecuada el estudio de los modelos de void a partir de observaciones del fondo cósmico de radiación, es entonces necesario trabajar con un modelo que contemple la evolución de dos fluidos no comóviles (materia y radiación) en conjunto con la propia geometría.

\subsubsection{Un modelo de dos fluidos no comóviles}

Teniendo en cuenta los argumentos expuestos en la sección anterior, un código numérico para calcular la evolución de un modelo de dos fluidos con simetría esférica (en donde la radiación y la materia son no comóviles) fue desarrollado recientemente por W. C. Lim, M. Regis y C. Clarkson [140]. ${ }^{5}$ El código integra las ecuaciones de evolución de ambos fluidos junto, con aquellas que determinan la dinámica de la geometría. De esta manera, se tienen en cuenta los efectos de la radiación en las etapas más tempranas de la evolución, en donde la densidad de

\footnotetext{
${ }^{4}$ Otra manera simple de entender este argumento es imaginar un void mucho más grande que la escala de Hubble actual, de forma tal que las regiones central y asintótica puedan ser representadas con modelos de FLRW independientes, cada una con un factor de expansión característico. Si la densidad de radiación se considera constante en el momento del desacople, los fotones del CMB tendrán entonces temperatura constante e igual en ambas regiones. Sin embargo, en tiempos tardíos la relación $T_{0}^{3} \propto \Omega_{m} h^{2}$ debe satisfacerse localmente y, en consecuencia, la diferencia de la temperatura de los fotones en cada una de las regiones será tan significativa cuanto lo sea la diferencia de densidades entre el centro y la parte asintótica [133].

Un último aspecto para tener en cuenta en el análisis de la libertad adicional que se tiene en el modelo al incorporar la radiación está relacionado con la especificación de la simetría esférica en términos del problema de Cauchy [133].

${ }^{5}$ COSMO-DRESS (COSMOlogical Dust and Radiation Evolution in a Spherically Symmetric Spacetime), disponible para su uso [140].
} 
fotones puede ser relevante. A diferencia de los casos en los que el contenido de materia es sólo polvo (descripto por la solución de LTB) o sólo radiación (descripto por la solución de Lemaître), las ecuaciones de Einstein no presentan soluciones exactas cuando se trabaja con ambas fuentes en un escenario no homogéneo. Por otra parte, debido a que los fluidos son no comóviles (el polvo sigue trayectorias geodésicas, pero la radiación no), el fluido total efectivo resulta anisótropo.

El modelo presentado en [140] está basado en dos hipótesis importantes:

1. los bariones y la materia oscura son descriptos mediante un único fluido con presión nula (una aproximación significativa, dado que en la época del desacople la distribución de bariones sufre los efectos producidos por el scattering Compton de los fotones); y

2. la radiación es modelada como un fluido perfecto (esto es, sin considerar los momentos multipolares de orden superior provenientes de la ecuación de Einstein-Boltzmann, como es la presión anisótropa de la radiación, que están lejos de ser despreciables en un espaciotiempo no homogéneo).

En relación al primer punto, un modelo más preciso sería aquel que describa la evolución de tres fluidos no comóviles, contemplando así la evolución de materia bariónica y materia oscura en forma independiente. El segundo punto es justamente el que motiva el trabajo presentado en este capítulo. En colaboración con C. Clarkson, M. Regis y W. C. Lim, nos propusimos generalizar el código numérico que modela la evolución de dos fluidos no homogéneos (esto es, polvo y radiación) llevando en cuenta los efectos de la presión anisótropa de la radiación no homogénea sobre la evolución de la geometría.

En las secciones siguientes, presentaremos el marco teórico en el que el modelo de dos fluidos fue desarrollado en [140], y el tratamiento necesario para incorporar en la dinámica la presión anisótropa de la radiación. La evolución de dicha cantidad se estudiará en el marco de la teoría cinética relativista. La implementación numérica del código, así como la comparación de los resultados obtenidos para los casos con y sin presión anisótropa, serán discutidos hacia el final del capítulo. Esta generalización del modelo forma parte del aporte original de esta tesis.

\subsection{Descripción del modelo de dos fluidos no homogéneos}

\subsubsection{Caracterización de la geometría}

La evolución de los dos fluidos no homogéneos es descripta en el formalismo de tétradas, a través de un conjunto completo de ecuaciones que garantiza la existencia de una métrica y una conexión afín para la descripción del problema [5]. Entre las ventajas que ofrece utilizar este formalismo, cabe resaltar que las ecuaciones de evolución resultan lineales, tanto en las derivadas temporales como en las espaciales. Una introducción detallada sobre el formalismo de tétradas en modelos cosmológicos puede encontrarse en el Apéndice A.

El elemento de línea que describe un espacio-tiempo con simetría esférica es

$$
\mathrm{d} s^{2}=-N^{2} \mathrm{~d} \tau^{2}+\left(\alpha_{\|}\right)^{-2} \mathrm{~d} r^{2}+\left(\alpha_{\perp}\right)^{-2}\left(\mathrm{~d} \theta^{2}+\operatorname{sen}^{2} \theta \mathrm{d} \varphi^{2}\right),
$$

con $N, \alpha_{\|}$y $\alpha_{\perp}$ funciones que dependen de $\tau$ y $r$. El movimiento del fluido cosmológico total (polvo más radiación) es descripto en un sistema de referencia comóvil con el fluido de 
polvo, caracterizado por la velocidad $u^{a}=\mathrm{d} x^{a} / \mathrm{d} \tau$ con $u_{a} u^{a}=-1$. La tétrada de vectores ortonormales que caracteriza la geometría esférica resulta

$$
\mathbf{e}_{0}=N^{-1} \partial_{\tau}, \quad \mathbf{e}_{1}=\alpha_{\|} \partial_{r}, \quad \mathbf{e}_{2}=\alpha_{\perp} \partial_{\theta}, \quad \mathbf{e}_{3}=\frac{\alpha_{\perp}}{\operatorname{sen} \theta} \partial_{\varphi}
$$

Las variables cinemáticas (esto es el shear, la rotación y la aceleración), se escriben

$$
\begin{aligned}
\sigma_{\alpha \beta} & =\operatorname{diag}\left(-2 \sigma_{+}, \sigma_{+}, \sigma_{+}\right) \\
\omega_{\alpha \beta} & =0 \\
\dot{u}_{\alpha} & =0
\end{aligned}
$$

en donde la aceleración nula indica que estamos trabajando en un sistema de referencia comóvil con el fluido de materia. La curvatura espacial y las componentes del tensor de Weyl son

$$
\begin{aligned}
{ }^{3} S_{\alpha \beta} & =\operatorname{diag}\left(-2^{3} S_{+},{ }^{3} S_{+},{ }^{3} S_{+}\right), \\
E_{\alpha \beta} & =\operatorname{diag}\left(-2 E_{+}, E_{+}, E_{+}\right), \\
H_{\alpha \beta} & =0
\end{aligned}
$$

con

$$
\begin{aligned}
{ }^{3} R & =4 \mathrm{e}_{1} a-6 a^{2}+2\left(\alpha_{\perp}\right)^{2}, \\
{ }^{3} S_{+} & =-\frac{1}{3} \mathbf{e}_{1} a+\frac{1}{3}\left(\alpha_{\perp}\right)^{2}, \\
E_{+} & =H \sigma_{+}+\sigma_{+}^{2}+{ }^{3} S_{+}-\frac{1}{2} \pi_{+},
\end{aligned}
$$

en donde $a(t, r)$ es una función asociada a la conmutación espacial de la tétrada $\mathbf{e}_{\alpha}, \mathrm{y}$ forma parte del conjunto de las variables que determinan la evolución del modelo.

Las ecuaciones dinámicas de todas las funciones mencionadas anteriormente (detalladas en el apéndice A), se obtienen a partir de las ecuaciones de evolución del campo gravitacional en Relatividad General, mientras que las funciones $N$ y $\Omega_{\alpha}^{\beta}$ (esta última asociada a la rotación del sistema de referencia espacial $\left\{\mathbf{e}_{\alpha}\right\}$ respecto a la base de Fermi) quedan determinadas por la elección del sistema de referencia en el que estamos trabajando. En adelante, consideraremos $N=\mathrm{e}^{\tau}$ y $\Omega_{\alpha \beta}=0$.

\subsubsection{Caracterización de los fluidos}

Sean $T_{a b \text { (mat) }}$ y $T_{a b \text { (rad) }}$ los tensores energía-momento que caracterizan los fluidos de materia y radiación, respectivamente. Cada uno de ellos satisface la condición de conservación $\nabla_{b} T^{a b}=$ 0 . El fluido total efectivo queda caracterizado con el tensor-energía momento

$$
T_{a b}=T_{a b}^{(\mathrm{mat})}+T_{a b}^{(\mathrm{rad})}
$$

con

$$
\begin{aligned}
T_{a b}^{(\mathrm{mat})} & =\rho u_{a} u_{b}, \\
T_{a b}^{(\mathrm{rad})} & =\mu u_{a} u_{b}+2 q_{(a} u_{b)}+p\left(g_{a b}+u_{a} u_{b}\right)+\pi_{a b} .
\end{aligned}
$$


Las ecuaciones de estado para la materia y la radiación son, respectivamente, $p_{\text {mat }}=0$ (polvo) y $p_{\text {rad }}=\mu / 3$. Dado que los fluidos son no comóviles, las cantidades $\mu, p, q_{a}$ y $\pi_{a b}$ caracterizan al fluido de la radiación en el sistema de referencia de la materia. Si $v_{a}$ es la velocidad relativa entre los fluidos, las mismas cantidades en el sistema de referencia de la radiación se recuperan mediante las siguientes transformaciones [258]:

$$
\begin{aligned}
\tilde{\mu}= & \mu+\gamma^{2}\left[v^{2}(\mu+p)-2 q_{a} v^{a}+\pi_{a b} v^{a} v^{b}\right], \\
\tilde{p}= & p+\frac{1}{3} \gamma^{2}\left[v^{2}(\mu+p)-2 q_{a} v^{a}+\pi_{a b} v^{a} v^{b}\right], \\
\tilde{q}_{a}= & \gamma q_{a}-\gamma \pi_{a b} v^{b}-\gamma^{3}\left[(\mu+p)-2 q_{b} v^{b}+\pi_{b c} v^{b} v^{c}\right] v_{a} \\
& -\gamma^{3}\left[v^{2}(\mu+p)-\left(1+v^{2}\right) q_{b} v^{b}+\pi_{b c} v^{b} v^{c}\right] u_{a}, \\
\tilde{\pi}_{a b}= & \pi_{a b}+2 \gamma^{2} v^{c} \pi_{c(a}\left\{u_{b)}+v_{b)}\right\}-2 v^{2} \gamma^{2} q_{(a} u_{b)}-2 \gamma^{2} q_{(a} v_{b)} \\
& -\frac{1}{3} \gamma^{2}\left[v^{2}(\mu+p)+\pi_{c d} v^{c} v^{d}\right] h_{a b} \\
& +\frac{1}{3} \gamma^{4}\left[2 v^{4}(\mu+p)-4 v^{2} q_{c} v^{c}+\left(3-v^{2}\right) \pi_{c d} v^{c} v^{d}\right] u_{a} u_{b} \\
& +\frac{2}{3} \gamma^{4}\left[2 v^{2}(\mu+p)-\left(1+3 v^{2}\right) q_{c} v^{c}+2 \pi_{c d} v^{c} v^{d}\right] u_{(a} v_{b)} \\
& +\frac{1}{3} \gamma^{4}\left[\left(3-v^{2}\right)(\mu+p)-4 q_{c} v^{c}+2 \pi_{c d} v^{c} v^{d}\right] v_{a} v_{b},
\end{aligned}
$$

con

$$
\tilde{u}_{a}=\gamma\left(u_{a}+v_{a}\right), \quad \gamma=\left(1-v^{2}\right)^{-1 / 2}, \quad v_{a} u^{a}=0,
$$

y en donde el símbolo indica cantidades expresadas en un sistema de referencia comóvil con la radiación.

\subsubsection{Modelo sin presión anisótropa de la radiación}

Para el caso en el que la presión anisótropa de la radiación no es tenida en cuenta, se consideran las cantidades $\tilde{q}_{a}$ y $\tilde{\pi}_{a b}$ idénticamente nulas durante toda la evolución. Luego, de las transformaciones (5.17)-(5.20) con $v_{a}=(0, v, 0,0)$ resulta

$$
p=\frac{\mu}{3}, \quad q_{a}=(0, q, 0,0), \quad \pi_{a b}=\operatorname{diag}\left(0,-2 \pi_{+}, \pi_{+}, \pi_{+}\right),
$$

con

$$
q=\frac{4}{3} \frac{\mu v}{\left(1+\frac{v^{2}}{3}\right)}, \quad \pi_{+}=-\frac{4}{9} \frac{\mu v^{2}}{\left(1+\frac{v^{2}}{3}\right)} .
$$

Las variables que se eligen para describir la evolución en este caso son

$$
\alpha_{\|}, \alpha_{\perp}, a, H, \sigma_{+}, \rho, \mu, v
$$


y las ecuaciones de evolución resultan $[140]^{6}$

$$
\begin{aligned}
\mathbf{e}_{0} \alpha_{\|} & =\left(-H+2 \sigma_{+}\right) \alpha_{\|}, \\
\mathbf{e}_{0} \alpha_{\perp} & =-\left(H+\sigma_{+}\right) \alpha_{\perp}, \\
\mathbf{e}_{0} H & =-H^{2}-2 \sigma_{+}^{2}-\frac{1}{6} \rho-\frac{1}{6}(\mu+3 p), \\
\mathbf{e}_{0} \sigma_{+} & =-3 H \sigma_{+}+\frac{1}{3} \mathbf{e}_{1} a-\frac{1}{3}\left(\alpha_{\perp}\right)^{2}-\frac{4}{9} \frac{\mu v^{2}}{\left(1+\frac{v^{2}}{3}\right)}, \\
\mathbf{e}_{0} a & =\left(-H+2 \sigma_{+}\right) a-\mathbf{e}_{1}\left(H+\sigma_{+}\right), \\
\mathbf{e}_{0} \rho & =-3 H \rho \\
\mathbf{e}_{0} \mu & =-\frac{4}{3} \frac{v}{\left(1+\frac{v^{2}}{3}\right)} \mathbf{e}_{1} \mu-\frac{4}{3} \frac{\left(1-\frac{v^{2}}{3}\right)}{\left(1+\frac{v^{2}}{3}\right)^{2}} \mu \mathbf{e}_{1} v \\
\mathbf{e}_{0} v & =-\frac{\left(1-v^{2}\right)^{2}}{4 \mu\left(1-\frac{v^{2}}{3}\right)} \mathbf{e}_{1} \mu-\frac{8}{9} \frac{v^{2} \mathbf{e}_{1} v}{\left(1+\frac{v^{2}}{3}\right)\left(1-\frac{v^{2}}{3}\right)}-\frac{v\left(1-v^{2}\right)}{\left(1-\frac{v^{2}}{3}\right)}\left[-2 \sigma_{+}+\frac{2}{3} v a\right] .
\end{aligned}
$$

con los vínculos

$$
\begin{aligned}
\left(C_{G}\right) & =0=3 H^{2}+\frac{1}{2} 3-3 \sigma_{+}^{2}-\rho-\mu \\
\left(C_{C}\right)_{1} & =0=-2 \mathbf{e}_{1}\left(H+\sigma_{+}\right)+6 a \sigma_{+}+\frac{4}{3} \frac{\mu v}{\left(1+\frac{v^{2}}{3}\right)}, \\
(\operatorname{def} a) & =0=\left(\mathbf{e}_{1}-a\right) \alpha_{\perp} .
\end{aligned}
$$

El modelo caracterizado por esta evolución fue estudiado en [140] para diferentes distribuciones no homogéneas de materia y radiación.

\subsubsection{Momentos multipolares de la radiación}

Dado que una distribución no homogénea de radiación da lugar a componentes anisótropas del fluido, los momentos multipolares de la radiación pueden jugar un rol importante en la dinámica del modelo [261]. El objetivo del trabajo que presentamos en este capítulo es generalizar la evolución de los fluidos presentada anteriormente para el caso en el que la presión anisótropa de la radiación es tenida en cuenta (esto es, cuando $\tilde{\pi} \neq 0$ ). La cantidad $\pi_{+}$se transforma entonces en una variable independiente, y el sistema de ecuaciones (5.25)(5.32) debe ser completado con una ecuación de evolución adicional. Dicha ecuación puede obtenerse a partir de un tratamientoo covariante de la teoría cinética relativista $[5,258] .{ }^{7}$

La teoría cinética relativista $[264,265,266,267]$ provee una descripción autoconsistente a nivel microscópico de un gas de partículas para un amplio rango de escenarios. En particular, permite estudiar la transición de un gas de fotones de un estado de equilibrio hidrodinámico (acoplado con la materia) a un estado de fluido libre. Esta transición es caracterizada por la evolución del camino libre medio de los fotones, y puede ser descripta apropiadamente por

\footnotetext{
${ }^{6} \mathrm{El}$ sistema de ecuaciones de evolución para el caso general de $n$ fluidos puede encontrarse en [259, 260].

${ }^{7}$ Un tratamiento alternativo, basado en la integración de las trayectorias de los fotones, ha sido desarrollado en $[262,263]$.
} 
el scattering de Thompson no relativista [268, 269]. El número de fotones contenido en un elemento de volumen $d V$, con coordenadas $x^{i}$ y momento $p^{a}$ en el volumen del espacio de momentos $\pi$, viene dado por [5]

$$
\mathrm{d} N=f\left(x^{i}, p^{a}\right)\left(-p_{a} u^{a}\right) \pi \mathrm{d} V,
$$

en donde $f\left(x^{i}, p^{a}\right)$ es la función de distribución de los fotones en el espacio de fases [264] y $u^{a}$ es la cuadri-velocidad del sistema de referencia. El factor de redshift, $\left(-p_{a} u^{a}\right)$, independiza a la función escalar $f$ de la elección de dicho sistema.

La tasa de cambio de $f$ está dada por la generalización relativista de la ecuación de Boltzmann, esto es,

$$
L(f)=C[f],
$$

en donde el operador de Louville dado por

$$
L(f)=\frac{\partial f}{\partial x^{i}} \frac{\mathrm{d} x^{i}}{\mathrm{~d} v}+\frac{\partial f}{\partial p^{a}} \frac{\mathrm{d} p^{a}}{\mathrm{~d} v}=0
$$

describe la variación de $f$ con el parámetro de distancia $d v$ a lo largo de las geodésicas que caracterizan el movimiento de las partículas. El término de colisiones entre las partículas, $C[f]$, puede ser considerado nulo si se quiere describir un escenario posterior al desacople entre los electrones y los fotones (es decir, que no se tienen en cuenta las variaciones en $f$ debidas a procesos de emisión, absorción y/o scattering de partículas).

El tensor de energía-momento de la radiación es

$$
T_{R}^{a b}=\int_{T_{x}} p^{a} p^{b} f \mathrm{~d} V
$$

en donde $\mathrm{d} V$ representa el elemento de volumen en el espacio de momento tangente, $T_{w}[5]$. El tensor $T_{R}^{a b}$ satisface las ecuaciones de conservación $\nabla_{b} T_{R}^{a b}=0$ y forma parte del tensor de energía-momento total, $T^{a b}$ (este último es el que determina la curvatura del espacio-tiempo a través de las ecuaciones de Einstein).

La función de distribución de los fotones puede ser escrita de manera covariante en el formalismo $1+3$ [270, 271], a partir de una descomposición angular en armónicos esféricos. Esta descomposición da lugar a un sistema de ecuaciones de evolución, acopladas en forma jerárquica, para los momentos multipolares de la radiación. En particular, la ecuación de evolución para el momento cuadrupolar, $\pi^{a b}$, resulta [5]

$$
\begin{aligned}
\dot{\pi}^{<a b>}+4 H & \pi^{a b}+\frac{8}{15} \mu \sigma^{a b}+\frac{2}{5} \tilde{\nabla}^{<a} q^{b>}+\frac{8 \pi}{35} \tilde{\nabla}_{c} \Pi^{a b c} \\
+2 \dot{u}^{<a} q^{b>}+2 \omega^{c} \eta_{c d} & <a \\
& \pi^{b>d}+\frac{2}{7} \sigma_{c}<a \pi^{b>c}-\frac{32 \pi}{315} \sigma_{c d} \pi^{a b c d}=0 .
\end{aligned}
$$

Esta ecuación, escrita en el formalismo ortogonal de tétradas en el que estamos trabajando, toma la forma

$$
\mathbf{e}_{0} \pi_{+}=-\frac{4}{3}\left(3 H \pi_{+}+\frac{2}{5} \mu \sigma_{+}\right)+\frac{2}{3}\left(\frac{1}{5} \mathbf{e}_{1}+\dot{u}\right) q+\frac{2}{7} \sigma_{+} \pi_{+},
$$

en donde los momentos multipolares de orden superior, $\Pi^{a b c}$ y $\Pi^{a b c d}$, no han sido tenidos en cuenta. Esta última hipótesis nos permite modelar de forma simplificada la evolución de la presión anisótropa de la radiación, pero debe ser levantada en el caso en que se quiera incluir en el modelo los momentos multipolares de orden superior. 


\subsubsection{Modelo de dos fluidos generalizado}

Las cantidades que caracterizan el fluido de radiación, cuando la presión anisótropa es tenida en cuenta, se derivan de las ecuaciones (5.17)-(5.20) con $\tilde{\pi} \neq 0$. En tal caso $\pi_{+}$pasa a ser una variable independiente en la evolución, y resulta

$$
q=\frac{2}{3}\left[\frac{2 \mu-3 \pi_{+}}{\left(1+v^{2}\right)}\right] .
$$

El modelo de dos fluidos no homogéneos queda ahora completamente caracterizado por las variables de evolución

$$
\alpha_{\|}, \alpha_{\perp}, H, \sigma_{+}, a, \rho, \mu, q, \pi_{+} .
$$

Notemos que en este caso elegimos trabajar con la variable $q$ (en lugar de $v$ ) para simplificar el cálculo numérico.

El sistema de ecuaciones diferenciales parciales que gobierna la dinámica del modelo se derivan de las expresiones (A.38)-(A.55), más la ecuación (5.41) para la evolución de la presión anisótropa de la radiación. El sistema completo de ecuaciones de evolución resulta entonces

$$
\begin{aligned}
\mathbf{e}_{0} \alpha_{\|} & =\left(-H+2 \sigma_{+}\right) \alpha_{\|}, \\
\mathbf{e}_{0} \alpha_{\perp} & =-\left(H+\sigma_{+}\right) \alpha_{\perp}, \\
\mathbf{e}_{0} H & =-H^{2}-2 \sigma_{+}^{2}-\frac{1}{6} \rho-\frac{1}{6}(\mu+3 p), \\
\mathbf{e}_{0} \sigma_{+} & =-3 H \sigma_{+}+\frac{1}{3} \mathbf{e}_{1} a-\frac{1}{3}\left(\alpha_{\perp}\right)^{2}+\pi_{+}, \\
\mathbf{e}_{0} a & =\left(-H+2 \sigma_{+}\right) a-\mathbf{e}_{1}\left(H+\sigma_{+}\right), \\
\mathbf{e}_{0} \rho & =-3 H \rho \\
\mathbf{e}_{0} \mu & =-3 H(\mu+p)-\left(\mathbf{e}_{1}-2 a\right) q-6 \sigma_{+} \pi_{+}, \\
\mathbf{e}_{0} q & =\left(-4 H+2 \sigma_{+}\right) q-\mathbf{e}_{1} p+2\left(\mathbf{e}_{1}-3 a\right) \pi_{+}, \\
\mathbf{e}_{0} \pi_{+} & =-\frac{4}{3}\left(3 H \pi_{+}+\frac{2}{5} \mu \sigma_{+}\right)+\frac{2}{15} \mathbf{e}_{1} q+\frac{2}{7} \sigma_{+} \pi_{+},
\end{aligned}
$$

con los vínculos

$$
\begin{aligned}
\left(C_{G}\right) & =0=3 H^{2}+\frac{1}{2}^{3} R-3 \sigma_{+}^{2}-\rho-\mu, \\
\left(C_{C}\right)_{1} & =0=-2 \mathbf{e}_{1}\left(H+\sigma_{+}\right)+6 a \sigma_{+}+q, \\
(\operatorname{def} a) & =0=\left(\mathbf{e}_{1}-a\right) \alpha_{\perp} .
\end{aligned}
$$

Las ecuaciones (5.44)-(5.52) generalizan el modelo de dos fluidos no homogéneos propuesto en [140], para el caso en el que la presión anisótropa de la radiación es tenida en cuenta durante el cómputo de la evolución.

\subsubsection{Cálculo del redshift y de distancias cosmológicas}

Dado que estamos interesados en estudiar los efectos de la distribución no homogénea de la radiación sobre cantidades observables (y, en particular, los efectos de su presión anisótropa), detallaremos a continuación la forma de calcular el redshift y la distancia de luminosidad, medidos sobre el cono de luz pasado de un observador en el centro de simetría. Los lineamientos del cálculo fueron presentados originalmente en [140]. 
Consideremos un fotón incidiendo en el centro del void en el tiempo presente, denotado por $t=t_{0}$. La posición del fotón en cada instante de tiempo pasado se determina con la función $x_{\text {fotón }}(t)$. De la condición $\mathrm{d} s^{2}=0$ para las geodésicas nulas, resulta

$$
\frac{\mathrm{d}}{\mathrm{d} t} x_{\mathrm{fotón}}(t)=-\left.N \alpha_{\|}\right|_{\left(t, x_{\mathrm{fotón}}(t)\right)},
$$

en donde $\frac{\mathrm{d}}{\mathrm{d} t}$ indica la derivada a lo largo de la geodésica nula, y la condición inicial es $x_{\text {fotón }}\left(t_{0}\right)=0$.

Sea $k^{a}$ el 4-vector asociado a una geodésica nula radial incidente en el centro de simetría. De la condición $k_{a} k^{a}=0$, en el sistema de referencia ortonormal se tiene que

$$
k^{a}=\epsilon N^{-1} \frac{\partial}{\partial t}-\epsilon \alpha_{\|} \frac{\partial}{\partial r}=(\epsilon,-\epsilon, 0,0),
$$

en donde $\epsilon$ es la energía del fotón. Además, el vector $k^{a}$ satisface la ecuación de la geodésica nula, dada por

$$
k_{a ; b} k^{b}=0 .
$$

Las componentes $k_{0 ; b} k^{b}=0$ y $k_{1 ; b} k^{b}=0$ dan lugar a la misma ecuación de evolución para $\epsilon$, que puede escribirse como

$$
\left(N^{-1} \frac{\partial}{\partial t}-\alpha_{\|} \frac{\partial}{\partial r}\right) \ln \epsilon=N^{-1} \frac{\partial}{\partial t} \ln \alpha_{\|}+\alpha_{\|} \frac{\partial}{\partial r} \ln N .
$$

Suponiendo que $N=N(t)$, y utilizando la ecuación (5.44) para la evolución de $\alpha_{\|}$, obtenemos

$$
\left(N^{-1} \frac{\partial}{\partial t}-\alpha_{\|} \frac{\partial}{\partial r}\right) \ln \epsilon=-\left(H-2 \sigma_{+}\right) .
$$

La energía del fotón incidente queda parametrizada sobre la geodésica nula por la coordenada temporal, esto es, $\epsilon(t) \equiv \epsilon\left(t, x_{\text {fotón }}(t)\right)$. Luego,

$$
\frac{\mathrm{d}}{\mathrm{d} t} \ln \epsilon(t)=-\left[N\left(H-2 \sigma_{+}\right)\right]_{\left(t, x_{\text {fotón }}(t)\right)} .
$$

El redshift es definido en términos de la energía del fotón incidente como

$$
z(t) \equiv \frac{\epsilon(t)}{\epsilon\left(t_{0}\right)}-1 .
$$

El valor presente de la energía del fotón, $\epsilon\left(t_{0}\right)$, es arbitrario y, en nuestro caso, se fija igual a 1 para la implementación numérica del código.

Por otra parte, la distancia de diámetro angular, $d_{A}$, es calculada a partir de la divergencia del campo de vectores nulos, esto es, $\theta=\nabla_{a} k^{a}$. Trabajando algebraicamente con esta expresión y la ecuación (5.59), se tiene

$$
\frac{\theta}{\epsilon}=-\left(N^{-1} \frac{\partial}{\partial t}-\alpha_{\|} \frac{\partial}{\partial r}\right) \ln \alpha_{\perp} .
$$

Si denotamos $A(t)$ al área de sección eficaz del haz de luz incidente, resulta

$$
N^{-1} \frac{\mathrm{d}}{\mathrm{d} t} \ln \sqrt{A}=\frac{\theta\left(t, x_{\text {fotón }}(t)\right)}{\epsilon\left(t, x_{\text {fotón }}(t)\right)},
$$



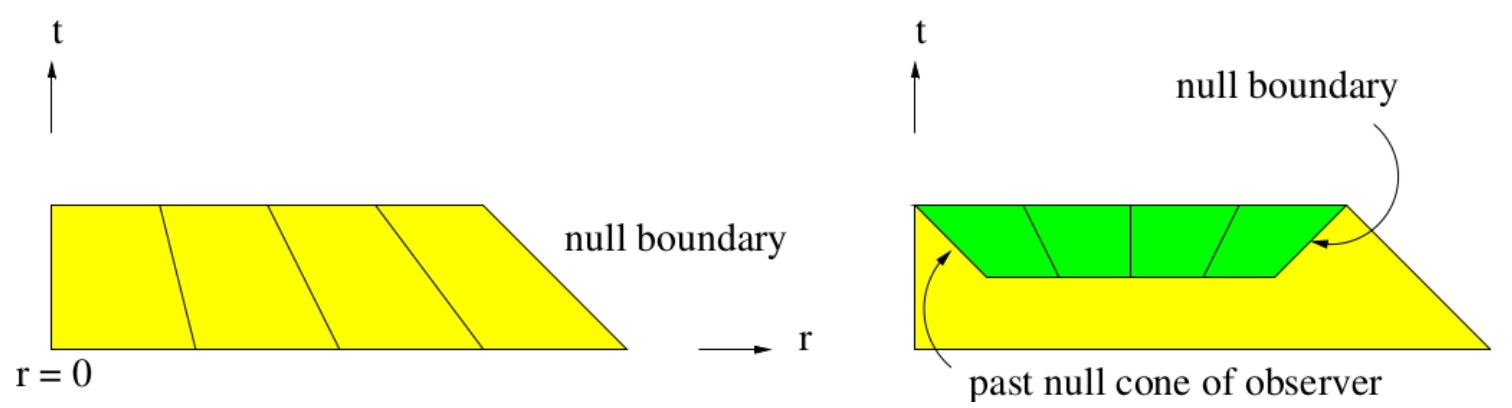

Figura 5.2: (extraída de [140]) Esquema de integración numérica para cada etapa de la evolución. Izquierda: en la primera parte de la evolución se computa la evolución del espacio-tiempo, integrando las ecuaciones desde $t_{*}$ (superficie de último scattering) hasta $t_{\text {hoy }}$. Derecha: en la segunda parte de la evolución se calculan las cantidades observables integrando la trayectoria de los fotones desde $t_{\text {hoy }}$ hacia el pasado sobre el cono de luz de un observador en el centro de simetría.

que implica entonces $\sqrt{A} \propto(1 / \alpha \perp)$. Teniendo en cuenta que la cantidad $\sqrt{A}$ es proporcional a la distancia de diámetro angular, resulta

$$
d_{A}=\left(\frac{1}{\alpha_{\perp}}\right)_{\text {centro }} .
$$

Finalmente, la distancia de luminosidad puede ser obtenida a partir de la relación de dualidad $d_{L}=(1+z)^{2} d_{A}$.

\subsection{Implementación numérica}

La evolución numérica del modelo se calcula en dos etapas, detalladas esquemáticamente en la figura 5.2. En la primera se computa la evolución de los fluidos y la geometría, integrando las ecuaciones (5.44)-(5.52) desde el momento del desacople de los fotones del fondo cósmico de radiación, $t=t_{*}$, hasta el tiempo presente, $t=t_{\text {hoy }}$. En la segunda parte de la evolución se calculan las cantidades observables que nos interesa estudiar (como la relación $d_{A}(z)$ ), integrando la trayectoria de los fotones desde $t_{\text {hoy }}$ hacia el pasado sobre el cono de luz de un observador en el centro del void en $t=t_{\text {hoy }}$.

La integración numérica de las ecuaciones diferenciales parciales que determina la evolución del modelo se realiza implementando el método de las líneas [249]. Para ello, se trabaja con una grilla uniforme discreta en la variable espacial $r$, y un esquema de derivación de cuarto orden. La integración numérica de las ecuaciones diferenciales ordinarias resultantes se lleva a cabo mediante un algoritmo de Runge-Kutta, también de cuarto orden, con las condiciones que detallaremos a continuación.

\subsubsection{Condiciones de borde y regularidad en el centro de simetría}

En el extremo interior de la grilla espacial se imponen condiciones de regularidad para las funciones de evolución. Dada la simetría esférica del problema, una solución suave en el centro de simetría implica que las todas las cantidades involucradas deben ser funciones 
pares o impares de la variable $r$. Teniendo en cuenta esta condición, y las características de las cantidades físicas que cada variable representa, la dependencia de las funciones con la coordenada radial en el centro debe ser de la forma

$$
\begin{aligned}
\alpha_{\|}, H, \rho, \mu & \rightarrow \sim 1, \\
\alpha_{\perp}, a & \rightarrow \sim r^{-1} \\
v, q & \rightarrow \sim r \\
\sigma_{+}, \pi_{+} & \rightarrow \sim r^{2} .
\end{aligned}
$$

Este comportamiento hace que algunos de los términos presentes en las ecuaciones de evolución se vuelvan singulares en el origen (en particular, aquellos que involucran a las funciones $\alpha_{\perp} \mathrm{y}$ a). Para intentar regularizar estos términos, implementamos el siguiente cambio de variables (sugerido originalmente en [140])

$$
\begin{aligned}
\alpha_{\perp} & \rightarrow A_{\perp} \equiv \frac{1}{\alpha_{\perp}}, \\
a & \rightarrow\left(a+\alpha_{\perp}\right) \equiv a+\alpha_{\perp} . .
\end{aligned}
$$

Asimismo, con el objeto de eliminar la mayor cantidad de términos con derivadas radiales a partir de las restricciones (5.53)-(5.55), consideramos el siguiente cambio de variables adicional

$$
\begin{aligned}
H & \rightarrow H_{\|} \equiv H-2 \sigma_{+}, \\
\sigma_{+} & \rightarrow H_{\perp}=H+\sigma_{+} .
\end{aligned}
$$

En el extremo derecho de la grilla espacial (correspondiente a la región asintóticamente homogénea del void) las condiciones de borde se especifican definiendo un sistema de coordenadas nulas $(T, X)$, con $\tau=T$ y $r=r(T, X)$, a partir de la técnica de acercamiento (o zooming) propuesta en [272]. Denominando $X_{\text {centro }}$ y $X_{\text {asintótico, }}$,espectivamente, a los extremos izquierdo y derecho de la grilla espacial,la coordenada $r$ se determina mediante la siguiente ecuación de evolución

$$
\partial_{T} r=\partial_{T} r_{\text {centro }}+\left(\partial_{T} r_{\text {asintótico }}-\partial_{T} r_{\text {centro }}\right) \frac{X-X_{\text {centro }}}{X_{\text {asintótico }}-X_{\text {centro }}},
$$

con la condición inicial $r=X$. Las funciones $\partial_{T} r_{\text {centro }} \mathrm{y} \partial_{T} r_{\text {asintótico para la primer parte de }}$ la evolución (desde $t_{*}$ hasta $t_{\text {hoy }}$ ) se especifican como

$$
\partial_{T} r_{\text {centro }}=0, \quad \partial_{T} r_{\text {asintótico }}=-\left.N\left(\alpha_{\|}\right)\right|_{X_{\text {asintótico }}},
$$

mientras que para la segunda parte de la evolución resultan

$$
\partial_{T} r_{\text {centro }}=-N\left(\alpha_{\|}\right)_{\text {centro }}, \quad \partial_{T} r_{\text {asintótico }}=N\left(\alpha_{\|}\right)_{\text {asintótico }} \text {. }
$$

Las derivadas parciales en el sistema de ecuaciones (5.44)-(5.52) se transforman de $(\tau, r)$ a $(T, X)$ utilizando la regla de la cadena, esto es,

$$
\partial_{\tau}=\partial_{T}-\frac{\partial_{T} r}{\partial_{X} r} \partial_{X}, \quad \partial_{r}=\left(\partial_{X} r\right)^{-1} \partial_{X}
$$


Las ecuaciones de evolución escritas en términos de las nuevas variables, y las coordenadas $(T, X)$, resultan

$$
\begin{aligned}
& \partial_{T} \alpha_{\|}=-N \alpha_{\|} H_{\|}+\frac{\partial_{T} r}{\partial_{X} r} \partial_{X} \alpha_{\|}, \\
& \partial_{T} A_{\perp}=N A_{\perp} H_{\perp}+\frac{\partial_{T} r}{\partial_{X} r} \partial_{X} A_{\perp}, \\
& \partial_{T} H_{\|}=-N\left[H_{\|}^{2}-H_{\perp}^{2}+\left(a+\alpha_{\perp}\right)^{2}-2 \frac{\left(a+\alpha_{\perp}\right)}{A_{\perp}}\right. \\
& \left.+\frac{\rho}{2}+\frac{2}{3} \mu+2 \pi_{+}\right]+\frac{\partial_{T} r}{\partial_{X} r} \partial_{X} H_{\|} \\
& \partial_{T} H_{\perp}=-N\left[\frac{3}{2} H_{\perp}^{2}-\frac{1}{2}\left(\left(a+\alpha_{\perp}\right)^{2}-2 \frac{\left(a+\alpha_{\perp}\right)}{A_{\perp}}\right)+\frac{\mu}{6}-\pi_{+}\right]+\frac{\partial_{T} r}{\partial_{X} r} \partial_{X} H_{\perp} \\
& \partial_{T}\left(a+\alpha_{\perp}\right)=-N\left[H_{\perp}\left(a+\alpha_{\perp}\right)+\frac{q}{2}\right]+\frac{\partial_{T} r}{\partial_{X} r} \partial_{X}\left(a+\alpha_{\perp}\right), \\
& \partial_{T} \rho=-N\left(H_{\|}+2 H_{\perp}\right) \rho+\frac{\partial_{T} r}{\partial_{X} r} \partial_{X} \rho, \\
& \partial_{T} \mu=N\left[2 \pi\left(H_{\|}-H_{\perp}\right)-\frac{4 \mu}{3}\left(H_{\|}+2 H_{\perp}\right)\right. \\
& \left.-\frac{\alpha_{\|}}{\partial_{X} r} \partial_{X} q+2 q\left(a+\alpha_{\perp}\right)-2 \frac{q}{A_{\perp}}\right]+\frac{\partial_{T} r}{\partial_{X} r} \partial_{X} \mu \\
& \partial_{T} q=-N\left[2 q\left(H_{\|}+H_{\perp}\right)+\frac{1}{3} \frac{\alpha_{\|}}{\partial_{X} r} \partial_{X} \mu-2 \frac{\alpha_{\|}}{\partial_{X} r} \partial_{X} \pi_{+}\right. \\
& \left.+6 \pi_{+}\left(a+\alpha_{\perp}\right)-6 \frac{\pi_{+}}{A_{\perp}}\right]+\frac{\partial_{T} r}{\partial_{X} r} \partial_{X} q \\
& \partial_{T} \pi_{+}=N\left[\frac{8 \mu}{45}\left(H_{\|}-H_{\perp}\right)-\frac{2}{7} \pi_{+}\left(5 H_{\|}+9 H_{\perp}\right)+\frac{2}{15} \frac{\alpha_{\|}}{\partial_{X} r} \partial_{X} q\right]+\frac{\partial_{T} r}{\partial_{X} r} \partial_{X} \pi_{+},(5.8
\end{aligned}
$$

con las siguientes restricciones

$$
\begin{aligned}
\left(C_{G}\right): & 0=H_{\perp}^{2}+2 H_{\perp} H_{\|}-3\left(a+\alpha_{\perp}\right)^{2}+2 \frac{\alpha_{\|}}{\partial_{X} r} \partial_{X}\left(a+\alpha_{\perp}\right)+4 \frac{\left(a+\alpha_{\perp}\right)}{A_{\perp}}-\rho-\mu,(5) \\
\left(C_{C}\right)_{1}: & 0=-2 \frac{\alpha_{\|}}{\partial_{X} r} \partial_{X} H_{\perp}+2\left(a+\alpha_{\perp}\right)\left(H_{\perp}-H_{\|}\right)-2 \frac{\left(H_{\perp}-H_{\|}\right)}{A_{\perp}}+q \\
\operatorname{def}(a): & 0=\left(a+\alpha_{\perp}\right) A_{\perp}+\frac{\alpha_{\|}}{\partial_{X} r} \partial_{X} A_{\perp}-1 .
\end{aligned}
$$

Si bien las restricciones (5.87) y (5.89) no entran directamente en el cómputo de la evolución, se utilizan para eliminar algunas de las derivadas radiales en el sistema (5.78)-(5.86), así como también para tener un test de precisión adicional del código numérico.

Notemos que el cambio de variables implementado, si bien simplifica considerablemente el sistema de ecuaciones diferenciales parciales, no regulariza completamente los términos problemáticos en el origen (en particular, aquellos que involucran los cocientes $\left(a+\alpha_{\perp}\right) / A_{\perp}$, $q / A_{\perp}$ y $\left.\pi_{+} / A_{\perp}\right)$. Para evitar problemas numéricos al computarlos, trabajaremos con la grilla espacial corrida media unidad del origen [140]. 


\subsubsection{Condiciones iniciales}

Para implementar la primera etapa de la evolución, las condiciones iniciales son especificadas en $t_{*}$. Como mencionamos en la introducción del capítulo, la caracterización el perfil de void se realiza especificando las diferentes funciones en el centro de simetría y en la región externa asintóticamente homogénea (en adelante, señaladas como "centro" y "asintótica", respectivamente). Supondremos que localmente las líneas de universo en dichos puntos siguen una evolución tipo FLRW con curvatura positiva. ${ }^{8}$

Los fluidos no homogéneos son representados por perfiles gaussianos con un ancho característico correspondiente a $z \sim 1$, que conectan los dos modelos de FLRW mencionados anteriormente. Esto es,

$$
\Omega_{m, r}\left(t_{*}\right)=\Omega_{m, r}^{(\text {asintótica) }}\left(t_{*}\right)+\left[\Omega_{m, r}^{(\text {centro })}\left(t_{*}\right)-\Omega_{m, r}^{(\text {asintótica) }}\left(t_{*}\right)\right] \exp \left(-\frac{r^{2}}{w_{m, r}^{2}}\right)
$$

en donde los subíndices $m$ y $r$ indican, respectivamente, materia y radiación. Los perfiles no homogéneos quedan entonces determinados por los seis parámetros iniciales

$$
\Omega_{r}^{\text {(asintótica) }}, \Omega_{r}^{\text {(centro) }}, \Omega_{m}^{\text {(asintótica) }}, \Omega_{m}^{\text {(centro) }}, w_{m}, w_{r}
$$

que se eligen en $t_{*}$ para obtener valores razonables de las siguiente cantidades físicas en $t_{\text {hoy }}\left(h_{0}^{\text {(centro) }} \simeq 0,7, \Omega_{m}^{\text {(asintótica) }}\left(t_{\text {hoy }}\right) \simeq 0,7, \Omega_{m}^{\text {(centro) }}\left(t_{\text {hoy }}\right) \simeq 0,16, T_{0}^{\text {(centro })}=2,725^{\circ} \mathrm{K}\right.$, $T_{0}^{(\text {asintótica })} \simeq 2,8^{\circ} \mathrm{K}, t_{\text {hoy }} \simeq 13 \times 10^{9}$ años $)$.

Una vez especificados los perfiles de densidad de materia y de radiación, la dependencia radial del resto de las variables se determina teniendo en cuenta la hipótesis de que cada línea de universo sigue localmente una evolución tipo FLRW. Luego,

- el parámetro de Hubble $H$ se coomputa, para cada r, a partir del producto $\left(H t_{*}\right)$ para modelos de FLRW abiertos [140]. Esto es,

$$
H t_{*}=\frac{1-\Omega_{r}^{1 / 2}}{\Omega_{k}}-\frac{1}{2} \frac{\Omega_{m}}{\Omega_{k}^{3 / 2}} \ln \left[\frac{\left(\frac{1}{2} \Omega_{m}+\Omega_{k}\right) \Omega_{k}^{-1 / 2}+1}{\frac{1}{2} \Omega_{m} \Omega_{k}^{-1 / 2}+\Omega_{r}^{1 / 2}}\right] ;
$$

- el valor inicial del shear se considera idénticamente nulo,

$$
\sigma_{+}=0, \quad\left(\text { es decir }, H_{\|}=H_{\perp}=H\right) ;
$$

- el fluido de radiación queda caracterizado inicialmente por las cantidades $\tilde{q}_{+} \equiv 0 \mathrm{y}$ $\tilde{\pi}_{+} \equiv 0$, que en el sistema de referencia comóvil con la materia resultan

$$
\begin{aligned}
q & =\frac{4}{3} \frac{v \mu}{\left(1+\frac{v^{2}}{3}\right)}, \\
\pi_{+} & =-\frac{4}{9} \frac{v^{2} \mu}{\left(1+\frac{v^{2}}{3}\right)} .
\end{aligned}
$$

\footnotetext{
${ }^{8}$ La elección de trabajar con modelos abiertos está motivada por los resultados encontrados en [133], que muestran que la tensión existente en el ajuste de diferentes conjuntos de datos puede ser aliviada al incluir los efectos de la radiación en dichos modelos.
} 
La libertad de gauge es utilizada para fijar la función $\alpha_{\|}$a un valor constante para todo $r$, que dependerá del valor máximo que puede alcanzar la velocidad relativa entre los fluidos. El algoritmo para calcular $\alpha_{\|}$y $v$ viene dado por el siguiente esquema recursivo:

1. $\alpha_{\|}$se calcula como

$$
\alpha_{\|}=\text {ratio } /\left(\frac{-6}{{ }^{3} R_{\text {asintótica }}}\right)^{1 / 2},
$$

con el valor inicial ratio $=1$, y la curvatura espacial en la región asintótica a $t_{*}$ dada por

$$
{ }^{3} R_{\text {asintótica }}=-6 \Omega_{k}^{\text {asintótica }} H^{\text {asintótica }} \text {. }
$$

2. Con el valor calculado para $\alpha_{\|}$, y las condiciones iniciales $\sigma_{+}=0, \pi_{+}=-\frac{4}{9} \frac{v^{2} \mu}{\left(1+\frac{v^{2}}{3}\right)}$ (i.e., $\left.\tilde{\pi}_{+}=0\right)$ y $q=\frac{4}{3} \frac{v \mu}{\left(1+\frac{v^{2}}{3}\right)}$, la restricción de Codazzi (ecuación (5.88)) resulta

$$
0=-2 \frac{\alpha_{\|}}{\partial_{X} r} \partial_{X}\left[3\left(H_{\|}+2 H_{\perp}\right)\right]+\frac{4}{3} \frac{v \mu}{\left(1+\frac{v^{2}}{3}\right)} .
$$

De la expresión anterior, se computa el valor de la cantidad $v /\left(1+\frac{v^{2}}{3}\right)$.

3. Luego, la velocidad del fluido de radiación es calculada como

$$
v=\frac{6\left(v /\left(1+\frac{v^{2}}{3}\right)\right)}{3+\sqrt{9-12\left(v /\left(1+\frac{v^{2}}{3}\right)\right)^{2}}},
$$

con

$$
\frac{v}{\left(1+\frac{v^{2}}{3}\right)}=\frac{\frac{\alpha_{\|}}{\partial_{X} r} \partial_{X}\left(H_{\|}+2 H_{\perp}\right)}{2 \mu} .
$$

El algoritmo se repite en forma recursiva, computando en cada paso

$$
\text { ratio }=\left(\frac{\bar{v}}{1+\frac{(\bar{v})^{2}}{3}}\right) /\left(\frac{\max |v|}{1+\frac{(\max |v|)^{2}}{3}}\right),
$$

en donde $\bar{v}$ es un parámetro fijo predeterminado. El criterio de corte viene dado por la condición $\max \left|v\left(t_{*}, r\right)\right|<\bar{v}$.

Por último, los perfiles iniciales de las variables $A_{\perp} \mathrm{y}\left(a+\alpha_{\perp}\right)$ se calculan resolviendo el sistema de ecuaciones diferenciales ordinarias dado por

$$
\begin{aligned}
\frac{\alpha_{\|}}{\partial_{X} r} \partial_{X} A_{\perp} & =1-\left(a+\alpha_{\perp}\right) A_{\perp}, \\
\frac{\alpha_{\|}}{\partial_{X} r} \partial_{X}\left(a+\alpha_{\perp}\right) & =-2\left(a+\alpha_{\perp}\right) / A_{\perp}+\frac{3}{2}\left(a+\alpha_{\perp}\right)^{2}+\frac{{ }^{3} R}{4},
\end{aligned}
$$

con condiciones iniciales $A_{\perp}=0$ y $\left(a+\alpha_{\perp}\right)=0$ en $r=0$, y utilizando en el origen el valor límite

$$
\lim _{r \rightarrow 0} \frac{\alpha_{\|}}{\partial_{X} r} \partial_{X}\left(a+\alpha_{\perp}\right)=\frac{{ }^{3} R}{12} .
$$




\subsection{Resultados y discusión}

Para estudiar los efectos de la presión anisótropa de la radiación no homogénea sobre la dinámica, comparamos la evolución numérica del modelo en los casos con y sin presión anisótropa de la radiación. Para ello, utilizamos iguales condiciones iniciales para determinar los perfiles de materia y radiación en $t_{*}$. La evolución se detiene, en ambos casos, cuando la temperatura de los fotones en el centro del void desciende hasta el valor $T_{0}^{(\text {centro })}=2,725^{\circ} \mathrm{K}$.

Como primer resultado interesante, vemos que mientras que el caso con $\tilde{\pi}_{+}=0$ finaliza en un tiempo $t_{\text {hoy }}=13,2 \times 10^{9}$ años, la evolución en el caso con presión anisótropa de la radiación alcanza el tiempo $t_{\text {hoy }}=15,5 \times 10^{9}$. Es decir que, bajo las mismas condiciones iniciales, la evolución se retrasa si la presión anisótropa de la radiación es tenida en cuenta en la dinámica de la geometría. Por otra parte, el valor del parámetro de Hubble en el centro del void, para los casos con y sin presión anisótropa resulta, respectivamente, $h_{0}^{\text {centro }}=0,57 \mathrm{y}$ $h_{0}^{\text {centro }}=0,66$ (en total acuerdo con la diferencia observada en la edad del universo alcanzada en cada caso).

La comparación de algunas cantidades físicas relevantes (la distancia de diámetro angular, $d_{A}$, el parámetro de Hubble, $h$, y la densidad de materia, $\left.\Omega_{m}\right)$, calculadas sobre el cono de luz pasado de un observador central, se muestran en la figura 5.3. Podemos observar que, mientras $\Omega_{m}$ no presenta diferencias significativas en su perfil evolutivo, las curvas de $h$ y $d_{A}$ muestran comportamientos peculiares. El parámetro de Hubble evoluciona cualitativamente igual en ambos casos, pero las curvas están separadas una de la otra. Esta característica es consecuencia directa de la diferencia observada entre los valores obtenidos para $h_{0}$ en cada caso. Este resultado representa una herramienta para aliviar la tensión entre el valor de $H_{0}$ y las observaciones provenientes del CMB, existente en los modelos de void de LTB. Otra diferencia significativa puede apreciarse en la curva de la distancia de diámetro angular para $z \sim 1100$. En el caso con presión anisótropa la curva alcanza valores mayores en aproximadamente un $10 \%$ debido a los efectos de la presión anisótropa. Con esto, podemos concluir que efectivamente los efectos de la presión anisótropa de la radiación sobre la evolución de los dos fluidos son importantes y, en consecuencia, deben incluirse en el computo para un estudio completo del modelo.

Nuestros resultados confirman además que los modelos de void con dos fluidos pueden dar lugar a una reinterpretación de las evidencias observacionales de una expansión acelerada del universo sin la necesidad de introducir energía oscura y, por consiguiente, deben ser considerados seriamente como un primer paso en la construcción de modelos cosmológicos complejos. $^{9}$

El análisis anterior es el paso inicial para dar lugar a la exploración de parámetros que permitan describir diferentes conjuntos de datos en forma simultánea, como aquellos provenientes del fondo cósmico de radiación, SN tipo Ia y mediciones de $H_{0}$. Además de los tests convencionales, como la comparación de las curvas de $d_{A}(z)$, es importante considerar como paso siguiente los límites provenientes de la distorsión del espectro de cuerpo negro del fondo cósmico de radiación (debida al scattering de los fotones por gas ionizado, que en el caso del modelo no homogéneo con simetría esférica se encuentra en movimiento radial) ${ }^{10}$, así como aquellos que provienen de las oscilaciones acústicas de bariones [32]. ${ }^{11}$

\footnotetext{
${ }^{9}$ La incorporación eventual de multipolos de orden superior podrá ser considerada en versiones ulteriores del código.

${ }^{10}$ Este efecto fue discutido en una geometría simple, denominada "burbuja de Hubble", en [90].

${ }^{11}$ Un tratamiento apropiado de las escalas radial y transversal asociadas a las BAO en el modelo de LTB
} 

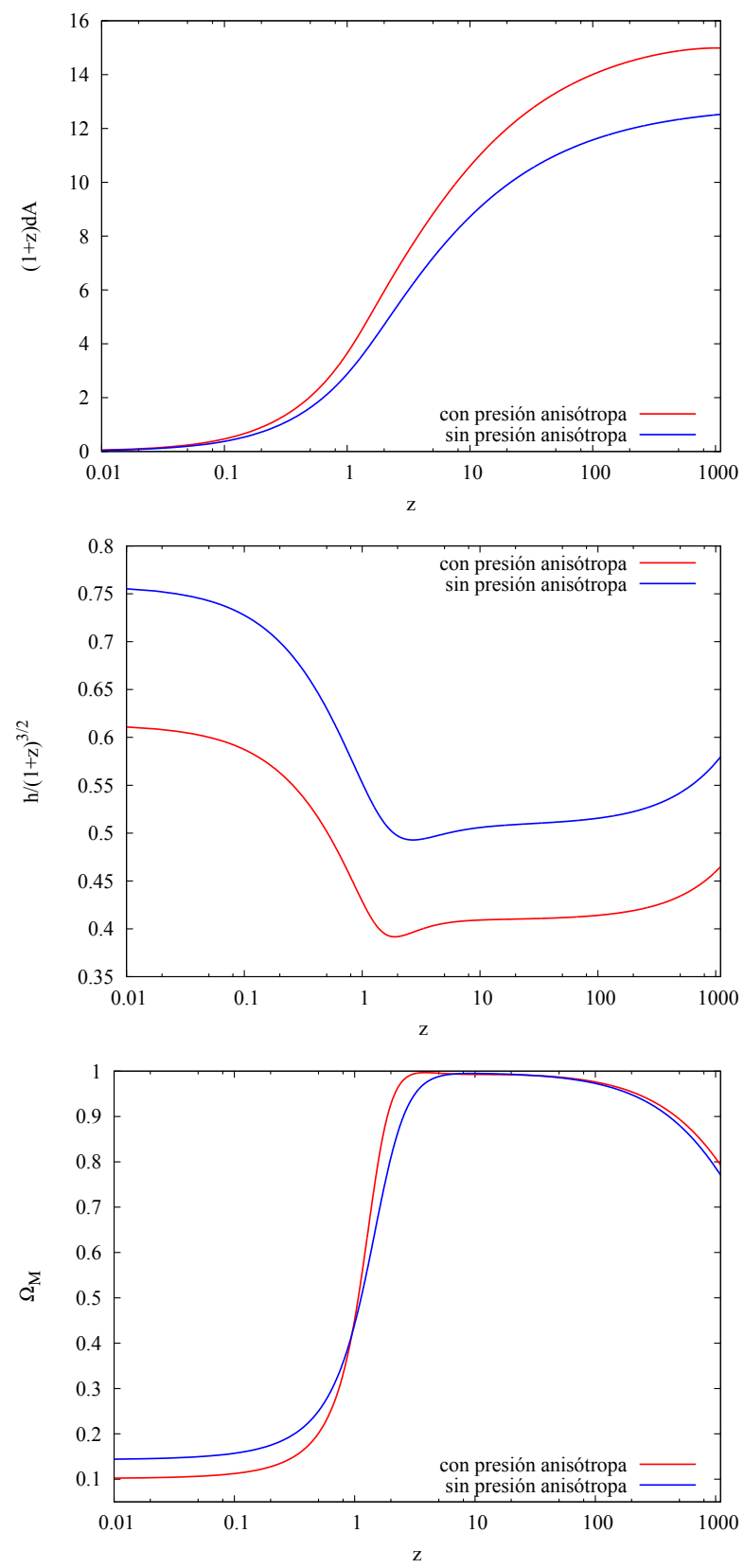

Figura 5.3: Observables cosmológicos en función del redshift para los casos con y sin presión anisótropa de la radiación. Las diferencias observadas en las curvas de $h$ y $d_{A}$ representan una herramienta para aliviar la tensión entre el valor de $H_{0}$ y las observaciones provenientes del CMB, existente en los modelos de void de LTB. 
Si bien el mecanismo para explicar la formación de voids es aún desconocido, el código numérico que hemos trabajado representa una primera exploración para abordar la construcción de problemas cosmológicos complejos. Entre las aplicaciones del modelo de dos fluidos no homogéneos, podemos destacar [140]:

- Las restricciones impuestas sobre los modelos de void.

Una de las principales limitaciones en los modelos de void con energía oscura [6] radica en el problema de combinar límites provenientes de observaciones del CMB junto con medidas locales de $H_{0}$ [256]. Como fue argumentado en [133], una evolución que contemple los efectos de una distribución no homogénea de la radiación podría levantar las restricciones sobre este tipo de modelos. Por otra parte, otras observaciones que dependen fuertemente del escenario cosmológico en el momento del desacople de los fotones con la materia, como el efecto Sunyaev-Zel'dovich y las evidencias de las oscilaciones acústicas de bariones en el espectro de potencias de las galaxias, pueden sufrir correcciones significativas si se tiene en cuenta una distribución no homogénea para el perfil primordial de la radiación, y los efectos que el mismo tiene sobre la evolución de la geometría de fondo.

- El estudio de los efectos relativistas durante la época del desacople de los fotones del fondo cósmico de radiación.

La evolución de las perturbaciones cosmológicas durante la época del desacople dan lugar a importantes desviaciones de un perfil gaussiano en el espectro primordial de potencias del CMB, así como también en el espectro de potencias de la materia en tiempos tardíos de la evolución [274]. Asimismo, la dinámica no lineal del plasma compuesto por materia bariónica y radiación, acoplado a la materia oscura a través de la gravedad, puede imponer correcciones significativas en las oscilaciones acústicas de bariones, y en el correspondiente pico observado en el espectro de potencias de las galaxias. En este contexto, los aspectos generales relativistas de las desviaciones a la teoría lineal canónica pueden ser estudiados con un modelo de dos fluidos, que incluso puede ser utilizado para testear la precisión de teorías de perturbaciones aplicadas sobre modelos homogéneos.

- El análisis de una etapa de nucleosíntesis primordial no homogénea.

La posibilidad de modelar la etapa de nucleosíntesis primordial con distribuciones no homogéneas para los cocientes entre bariones y fotones y/o neutrones y protones, en escalas comparables con el horizonte de difusión de partículas, ha sido estudiada en la literatura, generalmente considerando escenarios con simetría esférica [26]. El código numérico de dos fluidos no homogéneos puede ser utilizado entonces para estudiar el caso de distribuciones no homogéneas en la curvatura, y para acoplar la dinámica de la física de partículas con la expansión en un universo localmente no homogéneo.

(incorporando los efectos de anisotropías en la formación de estructura) está discutido en [273]. 


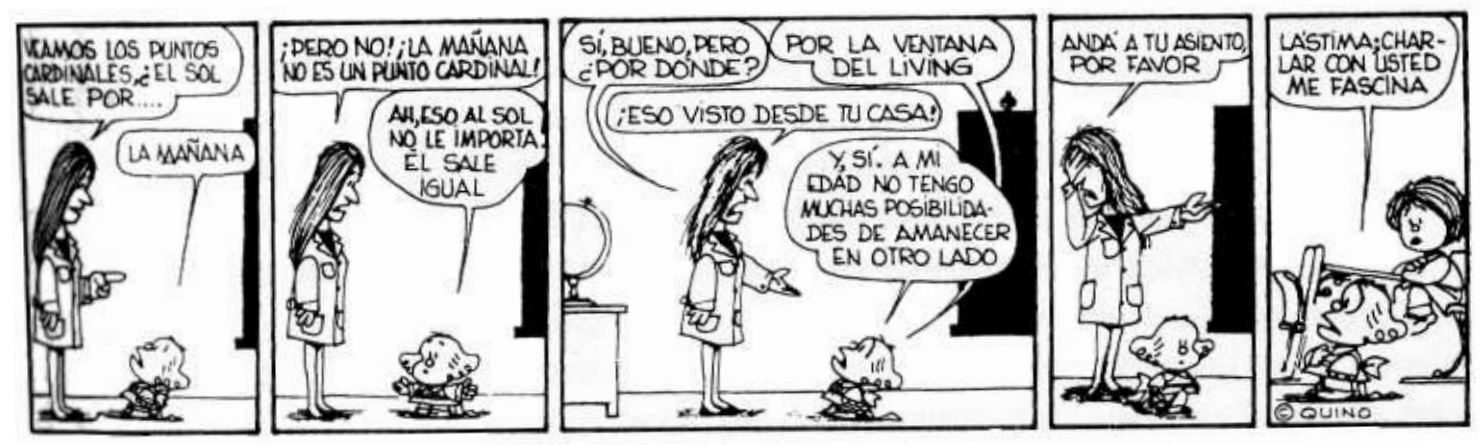

Mafalda (1964-1973)

Joaquín Salvador Lavado Tejón (Quino) 


\section{Capítulo 6}

\section{Conclusiones}

El modelo cosmológico estándar está construido sobre los fuertes pilares que representan las hipótesis de homogeneidad e isotropía. Basado en la métrica de FLRW, la más simple de las soluciones de las ecuaciones de Einstein, dicho modelo es capaz de reconstruir múltiples etapas de la evolución del universo, dando lugar a exitosos resultados a la hora ser cotejado con observaciones astronómicas de naturaleza diversa.

Sin embargo, el modelo no está libre de problemas. Si bien algunos de estos pueden ser resueltos de forma relativamente satisfactoria (por ejemplo, a partir de mecanismos tales como la inflación), el escenario cosmológico general no deja de presentar características extravagantes. Una de las más notorias es la naturaleza del contenido total de materia y energía presente en el universo. Mientras que tan sólo cerca del $5 \%$ del mismo es materia bariónica y radiación, aproximadamente el $25 \%$ es descripto por la llamada materia oscura, que no interactúa electromagnéticamente con la materia ordinaria y sería la que dio lugar a la formación de las estructuras que observamos hoy en día en el cosmos. Sin embargo, tal vez uno de los mayores paradigmas es el de la naturaleza del $70 \%$ del contenido de materia restante, descripto comúnmente por la llamada energía oscura: un fluido con características inusuales, no observadas en ningún otro material, y que sería además el responsable de la expansión acelerada del universo en la época actual.

Este panorama nos invita a ampliar el campo de investigación hacia escenarios cosmológicos más generales, y a revisar minusciosamente las hipótesis en la que está basada nuestra descripción del universo. En particular, la hipótesis de homogeneidad es una de las más fuertes y, sin embargo, de las más difíciles de testear observacionalmente. Una manera de abordar este problema es a través de la construcción de modelos cosmológicos no homogéneos. Si bien los mismos no representan un test directo para la hipótesis de homogeneidad, ofrecen una alternativa viable a la interpretación de observaciones astronómicas, sin la necesidad de recurrir a un contenido dominante de energía oscura en el universo. En esta dirección, las soluciones no homogéneas de las ecuaciones de Einstein han sido poco estudiadas, a pesar de su potencial para describir diferentes escenarios cosmológicos y astrofísicos.

Motivados por este escenario, nos propusimos como objetivo para nuestro trabajo (i) estudiar las propiedades genéricas de soluciones exactas no homogéneas de las ecuaciones de Einstein (en particular, aquellas con simetría esférica), y (ii) aplicar estas soluciones a problemas cosmológicos concretos.

Los trabajos que forman parte del aporte original de esta tesis abarcan los siguientes aspectos: 
1. El estudio del redshift drift de fuentes cercanas a partir del formalismo covariante $1+3$.

Gracias a las positivas perspectivas observacionales para los proximos años, el redshift drift ha cobrado especial interés por su potencial para distinguir modelos cosmológicos. En esta dirección, el análisis teórico que hemos presentado en el capítulo 3 ofrece la posibilidad de estudiar características distintivas de modelos cosmológicos, que podrían ser identificadas directamente en las observaciones (por ejemplo, un signo definido en la observación de $\left(\delta z / \delta t_{o}\right)$, o una dependencia direccional presente en modelos con menos simetrías).

Una aplicación adicional de la aproximación covariante que presentamos es la de poner cotas observacionales sobre las cantidades cinemáticas de los modelos. Esta tarea involucra, sin embargo, un análisis cuidadoso de la propagación de los errores observacionales sobre las cantidades involucradas en el cálculo. Si bien queda fuera de los alcances de este trabajo, este es un punto importante para abordar en un futuro próximo.

2. El análisis de la evolución de burbujas de vacío en fondos no homogéneos, como condiciones iniciales generales para el proceso de inflación.

En relación a las condiciones propicias para que el proceso de inflación tenga lugar, encontramos que ambientes no homogéneos (en particular, aquellos con radiación) da lugar a diferencias significativas en la evolución de regiones de vacío. En este sentido, los escenarios primordiales genéricos podrían ser restrictivos para la evolución de campos escalares dentro de la región inflacionaria, y deben ser analizadas minusciosamente en el marco de cada modelo inflacionario particular.

3. La generalización del modelo de void con dos fluidos, para el caso en el que la presión anisótropa de la radiación es tenida en cuenta en la evolución temprana de la geometría.

El modelo de dos fluidos presentado en el capítulo 5 fue estudiado para explorar si la inclusión de radiación puede tener efectos importantes sobre la evolución temprana de la geometría. Los resultados que presentamos muestran que las trayectorias de los fotones provenientes del fondo cósmico de radiación podrían sufrir correcciones importantes, incluso también si la presión anisótropa de la radiación (no nula en distribuciones no homogéneas) es tenida en cuenta. En este sentido, será un trabajo futuro cotejar cuidadosamente el modelo con conjuntos diversos de observaciones, con el objeto de cuantificar estos efectos de manera sistemática.

Asimismo, otros trabajos afines a los objetivos propuestos en esta tesis han sido desarrollados en forma paralela. Los mismos contribuyeron al estudio global de modelos alternativos al modelo cosmológico estándar. En particular, hemos abordado algunos problemas en el marco de las teorías de gravedad modificada $f(R)$, a saber:

- el estudio del redshift drift cosmográfico en modelos cosmológicos $f(R)$, como herramienta para poner restricciones sobre el espacio de parámetros libres de dichas teorías;

- la investigación de la estructura interna de estrellas de neutrones en el marco de teorías $f(R)$, como un camino para testear la Relatividad General en el régimen de campo fuerte.

Como mencionamos en la introducción de esta tesis, las soluciones no homogéneas de las ecuaciones de Einstein ha sido poco exploradas para dar respuesta a problemas cosmológicos 
concretos y, en este sentido, varias líneas de trabajo quedan abiertas para ser investigadas en el futuro. Entre estas, algunas de las que consideramos continuación natural del trabajo presentado en esta tesis son las siguientes:

- Varias propuestas para decidir por medio de la observación si el universo es homogéneo o no han sido presentadas en los últimos años [88, 89, 90, 91]. Las mismas se basan en el estudio de las geodésicas nulas del modelo de LTB, y ofrecerían información sobre perfiles radiales no homogéneos. La generalización de estos tests a casos con menos simetrías, con la posible inclusión de la presión de la radiación, aún no ha sido estudiada.

- En un universo no homogéneo, el efecto Sachs-Wolfe puede ser importante. Existen cálculos de este efecto sólo para el caso del modelo de LTB con un punto de observación situado en el centro de simetría [257]. La construcción de modelos más realistas invita a calcular este efecto para un punto de observación arbitrario, así como también para soluciones con presión y/o con menos simetrías.

- La presencia de regiones no homogéneas afecta, a través del "lensing gravitacional", al modo "B" de la radiación electromagnética del CMB, así como a la correlación entre los campos eléctrico y magnético. Tales modificaciones fueron calculadas en [275] para el caso de modelos de LTB. Es importante entonces generalizar este calculo a casos con presión y a modelos con menos simetría.

Para concluir, destacamos nuevamente que el modelo cosmológico estándar reconstruye la historia del universo en un exitoso acuerdo con las observaciones astronómicas actuales. En este sentido, lejos de pretender reemplazar completamente el modelo estándar, pero sí pensando en cambiar el paradigma cosmológico actual, nos interesa resaltar la importancia de la aplicación de soluciones no homogéneas para resolver problemas específicos presentes en diversas etapas de la evolución del universo. Esta área de estudio forma parte de la difícil tarea que implica revisar, ampliar y someter a tests el marco teórico en el que basamos nuestra descripción del universo, como paso necesario en el largo camino que conlleva la comprensión profunda del cosmos. 


\section{Apéndice A}

\section{Breve introducción a los formalismos covariantes}

Los formalismos covariantes poseen la ventaja de poder describir fenómenos físicos y propiedades geométricas mediante cantidades y relaciones tensoriales que permanecen invariantes ante transformaciones del sistema de referencia con el que se elija trabajar.

Presentaremos en este apéndice las definiciones básicas de las cantidades físicas necesarias para describir un modelo cosmológico, así como también las relaciones que dichas cantidades satisfacen en el marco de la Teoría de la Relatividad General. Nos limitaremos a incluir sólo aquellas definiciones y relaciones físicas utilizadas en el trabajo desarrollado en esta tesis. Una introducción completa a la descomposición covariante $1+3$ y a la formulación covariante de tétradas puede encontrarse en las referencias [5, 181, 258, 276, 277].

\section{A.1. Descomposición $1+3$}

La formulación $1+3$ está basada en la definición covariante de las variables físicas que describen un dado problema. Comenzaremos por caracterizar un escenario cosmológico con un espaciotiempo representado por una métrica $g_{a b}$ y un contenido de materia descripto por un tensor de energía-momento $T_{a b}$. La interacción entre la geometría y la materia, en el marco de la Teoría de la Relatividad General, obedece las ecuaciones de campo de Einstein dadas por

$$
G_{a b} \equiv R_{a b}-\frac{1}{2} R g_{a b}=T_{a b}-\Lambda g_{a b},
$$

con ecuaciones de conservación

$$
\nabla_{b} G^{a b}=0 \quad\left(\Rightarrow \nabla_{b} T^{b}=0\right)
$$

Para caracterizar un modelo cosmológico, definimos líneas de universo preferenciales comóviles con el fluido en cada punto. El 4-vector que representa la velocidad del fluido se define en forma unívoca como

$$
u^{a} \equiv \frac{\mathrm{d} x^{a}}{\mathrm{~d} \tau}, \quad \text { con } \quad u_{a} u^{a}=-1,
$$

en donde $\tau$ es el tiempo propio medido a lo largo de las geodésicas que determinan las trayectorias de los elementos de fluido. Los tensores de proyección paralela y ortogonal a $u^{a}$ se 
definen, respectivamente, como

$$
\begin{aligned}
U_{b}^{a} \equiv-u^{a} u_{b} & \Rightarrow U_{c}^{a} U_{b}^{c}=U_{b}^{a}, U_{a}^{a}=1, U_{a b} u^{b}=u_{a}, \\
h_{a b} \equiv g_{a b}+u_{a} u_{b} & \Rightarrow h_{c}^{a} h_{b}^{c}=h_{b}^{a}, h_{a}^{a}=3, h_{a b} u^{b}=0 .
\end{aligned}
$$

El elemento de volumen del espacio ortogonal en reposo respecto al sistema de referencia con velocidad $u^{a}$ se define como

$$
\eta_{a b c} \equiv u^{d} \eta_{d a b c} \quad \Rightarrow \eta_{a b c}=\eta_{[a b c]}, \eta_{a b c} u^{c}=0,
$$

en donde $\eta_{a b c d}$ es el elemento de volumen 4-dimensional, con $\eta_{a b c d}=\eta_{[a b c d]} \mathrm{y} \eta_{0123}=\sqrt{\left|\operatorname{det} g_{a b}\right|}$.

Teniendo en cuenta las proyecciones anteriores, se definen la derivada temporal covariante a lo largo de la dirección de $u^{a}$, denotada con el símbolo ${ }^{\circ}$, y la derivada covariante proyectada en el espacio ortogonal a $u^{a}$, señalada como $\tilde{\nabla}$. De esta forma, dado un tensor arbitrario $A_{c d}^{a b}$, resultan

$$
\begin{aligned}
\dot{A}^{a b}{ }_{c d} & =u^{e} \nabla_{e} A^{a b}{ }_{c d}, \\
\tilde{\nabla}_{e} A_{c d}^{a b} & =h^{a}{ }_{f} h^{b}{ }_{g} h^{p}{ }_{c} h^{q}{ }_{d} h^{r}{ }_{e} \nabla_{r} A^{f g}{ }_{p q} .
\end{aligned}
$$

Asimismo, la proyección ortogonal de vectores y la proyección ortogonal simétrica con traza nula de tensores de rango 2 se denotan, respectivamente, como

$$
v^{<a>}=h_{b}^{a} v^{b}, \quad A^{<a b>}=\left[h_{c}^{(a} h_{d}^{b)}-\frac{1}{3} h^{a b} h_{c d}\right] A^{c d},
$$

mientras que las proyecciones ortogonales de las derivadas temporales covariantes a lo largo de la dirección de $u^{a}$ (derivadas de Fermi) resultan

$$
\dot{v}^{<a>}=h_{b}^{a} \dot{v}^{b}, \quad \dot{A}^{<a b>}=\left[h_{c}^{(a} h_{d}^{b)}-\frac{1}{3} h^{a b} h_{c d}\right] \dot{A}^{c d} .
$$

La derivada covariante de la velocidad $u^{a}$ puede descomponerse en partes irreducibles, definidas a partir de sus propiedades de simetría, llamadas cantidades cinemáticas. La descomposición se escribe de la siguiente manera:

$$
\nabla_{a} u_{b}=-u_{a} \dot{u}_{b}+\tilde{\nabla}_{a} u_{b}=-u_{a} \dot{u}_{b}+\frac{1}{3} \Theta_{a b}+\sigma_{a b}+\omega_{a b},
$$

en donde

- $\dot{u}^{a} \equiv u^{b} \nabla_{b} u^{a}$ es el vector de aceleración relativista, que representa el grado de movimiento de la materia bajo fuerzas gravitacionales o de inercia;

- $\Theta \equiv \tilde{\nabla}_{a} u^{a}$ es el escalar de tasa de expansión de volumen del fluido (con $H=\Theta / 3$ el escalar de Hubble, para el caso de geometrías homogéneas e isótropas);

- $\sigma_{a b} \equiv \tilde{\nabla}_{<a} u_{b>}$ es el tensor simétrico de shear, que describe la tasa de distorsión del fluido de materia, con $\sigma_{a b}=\sigma_{(a b)}, \sigma_{a b} u^{b}=0, \sigma_{a}^{a}=0$, y traza nula); y

- $\omega_{a b} \equiv \tilde{\nabla}_{[a} u_{b]}$ es el tensor de vórtice $\left(\omega_{a b}=\omega_{[a b]}, \omega_{a b} u^{b}=0\right)$, que describe la rotación del fluido de materia. El vector de vórtice se define entonces como $\omega^{a} \equiv \frac{1}{2} \eta^{a b c} \omega_{c b}$. 
El significado de cada una de estas cantidades se infiere de la ecuación de evolución para el vector de posición relativa $\eta_{\perp}^{a} \equiv h_{b}^{a} \eta^{b}$, en donde $\eta^{a}$ es el vector de desviación de la familia de geodésicas fundamentales, es decir, aquellas que satisfacen $u^{b} \nabla_{b} \eta^{a}=\eta^{b} \nabla_{b} u^{a}$. Escribiendo $\eta_{\perp}^{a}=\delta \ell e^{a}$, con $e_{a} e^{a}=1$, encontramos que la distancia relativa $\delta \ell$ obedece la siguiente ecuación de propagación:

$$
\frac{(\dot{\delta} \ell)}{\delta \ell}=\frac{1}{3} \Theta+\left(\sigma_{a b} e^{a} e^{b}\right)
$$

(esto es, la ley de Hubble generalizada), y el vector de dirección relativa $e^{a}$ sigue la ecuación de propagación dada por

$$
\dot{e}^{<a>}=\left(\sigma_{b}^{a}-\left(\sigma_{c d} e^{c} e^{d}\right) h_{b}^{a}-\omega_{b}^{a}\right) e^{b},
$$

indicando la tasa de cambio de una posición observada en el cielo de galaxias distantes. ${ }^{1}$

Las ecuaciones de propagación para las cantidades cinemáticas (derivadas a partir de las identidades de Ricci, $\left.2 \nabla_{[a} \nabla_{b]} u^{c}=R_{a b d}^{c} u^{d}[5]\right)$ son:

- la ecuación de Raychaudhuri [278]

$$
\dot{\Theta}-\tilde{\nabla}_{a} \dot{u}^{a}=-\frac{1}{3} \Theta^{2}+\left(\dot{u}_{a} \dot{u}^{a}\right)^{2}-2 \sigma^{2}+2 \omega^{2}-\frac{1}{2}(\mu+3 p)+\Lambda ;
$$

- la ecuación de propagación para el shear

$$
\dot{\sigma}^{<a b>}-\tilde{\nabla}^{<a} \dot{u}^{b>}=-\frac{2}{3} \Theta \sigma^{a b}+\dot{u}^{<a} \dot{u}^{b>}-\sigma_{c}^{<a} \sigma^{b>c}-\omega^{<a} \omega^{b>}-\left(E^{a b}-\frac{1}{2} \pi^{a b}\right),
$$

y en donde $E_{a b}$ es la parte eléctrica del tensor de Weyl, definida como

$$
E_{a b} \equiv C_{a c b d} u^{c} u^{d}
$$

con $E_{a}^{a}=0, E_{a b}=E_{(a b)}$ y $E_{a b} u^{b}=0$, y el término de la presión anisótropa $\pi_{a b}$ se anula para el caso de fluidos perfectos; y

- la ecuación de propagación para el tensor de vórtice

$$
\dot{\omega}^{<a>}-\frac{1}{2} \eta^{a b c} \tilde{\nabla}_{b} \dot{u}_{c}=-\frac{2}{3} \Theta \omega^{a}+\sigma_{b}^{a} \omega^{b} .
$$

\footnotetext{
${ }^{1}$ Una manera de interpretar el sentido físico de las cantidades cinemáticas es analizar cuáles son los cambios sufridos por una esfera de fluido, asociados a cada una estas cantidades, al cabo de un intervalo infinitesimal de tiempo. A saber: la acción aislada de $\Theta$ transforma la esfera de fluido en una esfera similar, con diferente volumen, pero orientada en las mismas direcciones; la acción aislada de $\sigma_{a b}$ distorsiona la forma de la esfera, manteniendo su volumen constante y la dirección de sus ejes principales sin modificación; y la acción aislada de $\omega_{a b}$ da lugar a una rotación rígida de la esfera en torno a una de sus direcciones, que permanece fija (eje de rotación). La acción conjunta de estas cantidades, por su parte, modificará en general la dirección de los ejes principales y de rotación [181].
} 


\section{A.2. Tétradas en modelos cosmológicos}

La descomposición $1+3$ presentada en la sección anterior es inmediatamente transparente en términos de relaciones y cantidades físicas definidas covariantemente, con un significado físico y/o geométrico claro. Sin embargo, las ecuaciones involucradas no forman un conjunto completo que garantice siempre la existencia de una métrica y sus conexiones afines. Para tal fin, es conveniente abordar la descripción del modelo con el formalismo de tétradas, en el cual las conexiones afines están definidas en términos de los coeficientes de rotación espacial. Las relaciones obtenidas con este formalismo determinan un conjunto completo de ecuaciones para la caracterización de escenarios cosmológicos arbitrarios [5]. ${ }^{2}$

Una tétrada es una base de vectores unitarios, mutuamente ortogonales, $\left\{\mathbf{e}_{a}\right\}, a=$ $0,1,2,3$, que pueden ser escritos en términos de una base de coordenadas locales. Las componentes tétradas $e_{a}^{i}\left(x^{j}\right)$ se definen como

$$
\mathbf{e}_{a}=e_{a}^{i}\left(x^{j}\right) \frac{\partial}{\partial x^{i}} \quad \leftrightarrow \quad \mathbf{e}_{a}(f)=e_{a}^{i}\left(x^{j}\right) \frac{\partial f}{\partial x^{i}}, \quad e_{a}^{i}=\mathbf{e}_{a}\left(x^{i}\right),
$$

es decir, que la componente $i$ del vector $a$ es simplemente la derivada direccional de la coordenada $i$ en la dirección del vector $\mathbf{e}_{a}$. Esto puede ser pensado como un cambio de vectores base, en donde las componentes tensoriales se transforman como

$$
T^{a b}{ }_{c d}=e_{i}^{a} e^{b}{ }_{j} e_{c}^{k} e_{d}^{l} T^{i j}{ }_{k l},
$$

y las componentes inversas $e_{i}^{a}\left(x^{j}\right)$ se definen como

$$
e_{a}^{i} e^{a}{ }_{j}=\delta^{i}{ }_{j} \quad \leftrightarrow e_{a}^{i} e_{i}^{b}=\delta_{a}^{b} .
$$

Las componentes del tensor métrico resultan

$$
g_{a b}=g_{i j} e_{a}^{i} e_{b}^{j}=\mathbf{e}_{a} \cdot \mathbf{e}_{b}=\eta_{a b},
$$

con $\eta_{a b}=\operatorname{diag}(-1,1,1,1)$. La ecuación inversa

$$
g_{i j}\left(x^{k}\right)=\eta_{a b} e_{i}^{a}\left(x^{k}\right) e^{b}{ }_{j}\left(x^{k}\right)
$$

construye explícitamente las componentes coordenadas de la métrica a partir de las componentes tétradas $e_{i}^{a}\left(x^{j}\right)$. Los índices se manipulan entonces utilizando las métricas $g_{a b}=\eta_{a b} \mathrm{y}$ su inversa $g^{a b}=\eta^{a b}$.

Las funciones de conmutación asociadas a las tétradas son las cantidades $\gamma_{b c}^{a}\left(x^{i}\right)$, definidas por los conmutadores de los vectores base como

$$
\left[\mathbf{e}_{a}, \mathbf{e}_{b}\right]=\gamma_{a b}^{c}\left(x^{i}\right) \mathbf{e}_{c} \rightarrow \gamma_{b c}^{a}\left(x^{i}\right)=-\gamma_{c b}^{a}\left(x^{i}\right) .
$$

En términos de las componentes tétradas, resulta entonces

$$
\gamma_{b c}^{a}\left(x^{i}\right) e_{i}^{a}\left(e_{b}^{j} \partial_{j} e_{c}^{i}-e_{c}^{j} \partial_{j} e_{b}^{i}\right)=-2 e_{b}^{i} e_{c}^{j} \nabla_{[i} e^{a}{ }_{j]} .
$$

\footnotetext{
${ }^{2}$ En el caso general de geometrías anisótropas, únicamente los vectores tétrados pueden ser definidos en la forma covariante $1+3$, y esta descomposición pasa entonces a estar contenida dentro del formalismo de tétradas [5].
} 
Estas cantidades se anulan si y sólo si la base $\left\{\mathbf{e}_{a}\right\}$ es una base coordenada, es decir, si existen coordenadas locales $x^{i}$ tales que

$$
\mathbf{e}_{a}=\delta_{a}{ }^{i} \frac{\partial}{\partial x^{i}} \quad \text { sii } \quad\left[\mathbf{e}_{a}, \mathbf{e}_{b}\right]=0 \leftrightarrow \gamma_{b c}^{a}=0 .
$$

Las componentes de la conexión afín para las tétradas, $\Gamma_{b c}^{a}$ quedan definidas por las relaciones

$$
\nabla_{\mathbf{e}_{b}} \mathbf{e}_{a}=\Gamma_{a b}^{c} \mathbf{e}_{c} \quad \leftrightarrow \quad \Gamma_{a b}^{c}=e_{i}^{c} e_{b}^{j} \nabla_{j} e_{a}^{i},
$$

es decir, son las componentes $c$ de la derivada covariante del vector $a$ en la dirección $b$. Con esto, todas las derivadas covariantes pueden ser escritas de manera análoga a la forma tensorial usual. $^{3}$ En particular, teniendo en cuenta que $\mathbf{e}_{a}\left(g_{b c}\right)=0$ para $g_{a b}=\eta_{a b}$, se tiene

$$
\nabla_{a} g_{b c}=0 \quad \leftrightarrow \quad-\Gamma_{b a}^{d} g_{d c}-\Gamma_{c a}^{d} g_{b d}=0 \quad \leftrightarrow \quad \Gamma_{(a b) c}=0 .
$$

Para el caso de torsión nula, las relaciones tétradas resultan análogas a las relaciones de Christoffel usuales, es decir,

$$
\gamma_{b c}^{a}=-\left(\Gamma_{c b}^{a}-\Gamma_{c b}^{a}\right), \quad \Gamma_{a b c}=\frac{1}{2}\left(g_{a d} \gamma_{c b}^{d}-g_{b d} \gamma_{c a}^{d}+g_{c d} \gamma_{a b}^{d} .\right.
$$

Esto demuestra que los coeficientes de rotación y las funciones de conmutación son combinaciones lineales unas de las otras.

Las identidades de Jacobi $([X,[Y, z]]+[Y,[Z, X]]+[Z,[X, Y]]=0)$ para la base de vectores $\left\{\mathbf{e}_{a}\right\}$ resultan

$$
\mathbf{e}_{[a}\left(\gamma_{b c]}^{d}\right)+\gamma_{[a b}^{e} \gamma_{c] e}^{d}=0
$$

siendo esta la condición de integrabilidad para que las cantidades $\gamma_{b c}^{a}\left(x^{i}\right)$ sean las funciones de conmutación de ese conjunto de vectores.

Las componentes del tensor de Riemann, obtenidas a partir de las identidades de Ricci, tienen la forma

$$
R_{b c d}^{a}=\mathbf{e}_{c}\left(\Gamma_{b d}^{a}\right)-\mathbf{e}_{d}\left(\Gamma_{b c}^{a}\right)+\Gamma_{e c}^{a} \Gamma_{b d}^{e}-\Gamma_{e d}^{a} \Gamma_{b c}^{e}-\Gamma_{b e}^{a} \Gamma_{c d}^{e}
$$

y la contracción de los índices $a$ y $c$ da lugar a las ecuaciones de Einstein, dadas por

$$
R_{b d}=\mathbf{e}_{a}\left(\Gamma_{b d}^{a}\right)-\mathbf{e}_{b}\left(\Gamma_{b a}^{a}\right)+\Gamma_{e a}^{a} \Gamma_{b d}^{e}-\Gamma_{d e}^{a} \Gamma_{b a}^{e}=T_{b d}-\frac{1}{2} T g_{b d}+\Lambda g_{b d} .
$$

Para describir un modelo cosmológico, se elige $\mathbf{e}_{0}$ en la dirección tangente al flujo de materia $u^{a}$. Las componentes de la conexión $\Gamma_{b c}^{a}$ resultan

$$
\begin{aligned}
\Gamma_{\alpha 00} & =\dot{u}_{\alpha}, \\
\Gamma_{\alpha 0 \beta} & =\frac{1}{3} \Theta \delta_{\alpha \beta}+\sigma_{\alpha \beta}-\epsilon_{\alpha \beta \gamma} \omega^{\gamma}, \\
\Gamma_{\alpha \beta 0} & =\epsilon_{\alpha \beta \gamma} \Omega^{\gamma}, \\
\Gamma_{\alpha \beta \gamma} & =2 a_{[\alpha} \delta_{\beta] \gamma}+\epsilon_{\gamma \delta[\alpha} n_{\beta]}^{\delta}+\frac{1}{2} \epsilon_{\alpha \beta \delta} n_{\gamma}^{\delta},
\end{aligned}
$$

\footnotetext{
${ }^{3}$ Por ejemplo, se tiene

$$
\nabla_{a} T_{b c}=\mathbf{e}_{a}\left(T_{b c}\right)-\Gamma_{b a}^{d} T_{d c}-\Gamma_{c a}^{d} T_{b d}
$$
}

en donde, para una dada función $f, \mathbf{e}_{a}(f)=e_{a}^{i} \partial f / \partial x^{i}$ representa la derivada de $f$ en la dirección $\mathbf{e}_{a}$. 
en donde $\dot{u}^{\alpha}, \Theta, \sigma^{\alpha \beta}$ y $\omega^{\alpha}$ son las variables cinemáticas, $\Omega^{\alpha}$ indica la rotación del sistema de referencia espacial $\left\{\mathbf{e}_{\alpha}\right\}$ respecto a la base de Fermi, y las funciones $a^{\alpha}$ y $n_{\alpha \beta}=n_{(\alpha \beta)}$ (esta última simétrica) determinan los 9 coeficientes de rotación espacial, y están asociadas a la curvatura de las hipersuperficies $t=$ cte. Los conmutadores resultan

$$
\begin{aligned}
& {\left[\mathbf{e}_{0}, \mathbf{e}_{\alpha}\right]=\dot{u}_{\alpha} \mathbf{e}_{0}-\left[\frac{1}{3} \Theta \delta_{\alpha}^{\beta}+\sigma_{\alpha}^{\beta}+\epsilon_{\alpha \gamma}^{\beta}\left(\omega^{\alpha}+\Omega^{\alpha}\right)\right] \mathbf{e}_{\beta},} \\
& {\left[\mathbf{e}_{\alpha}, \mathbf{e}_{\beta}\right]=2 \epsilon_{\alpha \beta \gamma} \omega^{\gamma} \mathbf{e}_{0}+\left(2 a_{[\alpha} \delta_{\beta]}^{\gamma}+\epsilon_{\alpha \beta \delta} n^{\delta \gamma}\right) \mathbf{e}_{\gamma} .}
\end{aligned}
$$

en donde vemos que los coeficientes son, esencialmente, derivadas parciales de $N$ y $e_{\alpha}^{i}$.

La dinámica de un fluido gravitacional puede describirse mediante un conjunto de ecuaciones de evolución, más un grupo de ecuaciones que imponen restricciones sobre las funciones de evolución pero que no contienen derivadas con respecto a $\left\{\mathbf{e}_{\alpha}\right\}$. Este último grupo de ecuaciones puede expresarse como

$$
\begin{aligned}
& 0=\left(C_{1}\right)^{\alpha}=\left(\mathbf{e}_{\beta}-3 a_{\beta}\right)\left(\sigma^{\alpha \beta}\right)-\frac{2}{3} \delta^{\alpha \beta} \mathbf{e}_{\beta}(\Theta)-n^{\alpha}{ }_{\beta} \omega^{\beta}+q^{\alpha} \\
& +\epsilon^{\alpha \beta \gamma}\left[\left(\mathbf{e}_{\beta}+2 \dot{u}_{\beta}-a_{\beta}\right)\left(\omega_{\gamma}\right)-n_{\beta \delta} \sigma_{\gamma}^{\delta}\right], \\
& 0=\left(C_{2}\right)=\left(\mathbf{e}_{\alpha}-\dot{u}_{\alpha}-2 a_{\alpha}\right)\left(\omega^{\alpha}\right), \\
& 0=\left(C_{3}\right)^{\alpha \beta}=H^{\alpha \beta}+\left(\delta^{\gamma<\alpha} \mathbf{e}_{\gamma}+2 \dot{u}^{<\alpha}+a^{<\alpha}\right)\left(w^{\beta>}\right)-\frac{1}{2} n_{\gamma}^{\gamma} \sigma^{\alpha \beta}+3 n_{\gamma}^{<\alpha} \sigma^{\beta>\gamma} \\
& -\epsilon^{\gamma \delta<\alpha}\left[\left(\mathbf{e}_{\gamma}-a_{\gamma}\right)\left(\sigma_{\delta}^{\beta>}\right)+n_{\gamma}^{\beta>} \omega_{\delta}\right] \text {, } \\
& 0=\left(C_{4}\right)^{\alpha}=\left(\mathbf{e}_{\beta}-3 a_{\beta}\right)\left(E^{\alpha \beta}+\frac{1}{2} \pi^{\alpha \beta}\right)-\frac{1}{3} \delta^{\alpha \beta} \mathbf{e}_{\beta}(\mu)+\frac{1}{3} \Theta q^{\alpha}-\frac{1}{2} \sigma_{\beta}^{\alpha} q^{\beta}-3 \omega_{\beta} H^{\alpha \beta} \\
& -\epsilon^{\alpha \beta \gamma}\left[\sigma_{\beta \delta} H_{\gamma}^{\delta}-\frac{3}{2} \omega_{\beta} q_{\gamma}+n_{\beta \delta}\left(E_{\gamma}^{\delta}+\frac{1}{2} \pi_{\gamma}^{\delta}\right)\right], \\
& 0=\left(C_{5}\right)^{\alpha}=\left(\mathbf{e}_{\beta}-3 a_{\beta}\right)\left(H^{\alpha \beta}\right)+(\mu+p) \omega^{\alpha}+3 \omega_{\beta}\left(E^{\alpha \beta}-\frac{1}{6} \pi^{\alpha \beta}\right)-\frac{1}{2} n_{\beta}^{\alpha} q^{\beta} \\
& +\epsilon^{\alpha \beta \gamma}\left[\frac{1}{2}\left(\mathbf{e}_{\beta}-a_{\beta}\right)\left(q_{\gamma}\right)+\sigma_{\beta \delta}\left(E_{\gamma}{ }^{\delta}+\frac{1}{2} \pi_{\gamma}^{\delta}\right)-n_{\beta \delta} H_{\gamma} \delta\right], \\
& 0=\left(C_{J}\right)^{\alpha}=\left(\mathbf{e}_{\beta}-2 a_{\beta}\right)\left(n^{\alpha \beta}\right)+\frac{2}{3} \Theta \omega^{\alpha}+2 \sigma_{\beta}^{\alpha} \omega^{\beta}+\epsilon^{\alpha \beta \gamma}\left[\mathbf{e}_{\beta}\left(a_{\gamma}\right)-2 \omega_{\beta} \Omega_{\gamma}\right], \\
& 0=\left(C_{G}\right)^{\alpha \beta}={ }^{*} S^{\alpha \beta}+\frac{1}{3} \Theta \sigma^{\alpha \beta}-\sigma^{<\alpha}{ }_{\gamma} \sigma^{\beta>\gamma}-\omega^{<\alpha} \omega^{\beta>}+2 \omega^{<\alpha} \Omega^{\beta>} \\
& -\left(E^{\alpha \beta}+\frac{1}{2} \pi^{\alpha \beta}\right) \\
& 0=\left(C_{G}\right)={ }^{*} R+\frac{2}{3} \Theta^{2}-\left(\sigma_{\alpha \beta} \sigma^{\alpha \beta}\right)-4\left(\omega_{\alpha} \Omega^{\alpha}\right)-2 \mu-2 \Lambda,
\end{aligned}
$$


en donde

$$
\begin{aligned}
{ }^{*} S_{\alpha \beta} & =\mathbf{e}_{<\alpha}\left(a_{\beta>}\right)+b_{<\alpha \beta>}-\epsilon^{\gamma \delta}{ }_{<\alpha}\left(\mathbf{e}_{|\gamma|}-2 a_{|\gamma|}\right)\left(n_{\beta>\delta}\right), \\
{ }^{*} R & =2\left(2 \mathbf{e}_{\alpha}-3 a_{\alpha}\right)\left(a^{\alpha}\right)-\frac{1}{2} b_{\alpha}^{\alpha}, \\
b_{\alpha \beta} & =2 n_{\alpha \beta} n_{\beta}^{\gamma}-n_{\gamma}^{\gamma} n_{\alpha \beta} .
\end{aligned}
$$

En el caso de rotación nula, $\omega^{\alpha}=0$, la velocidad $u^{\alpha}$ resulta normal a la familia de hipersuperficies con $t=$ cte.

Las ecuaciones de evolución para las 9 funciones que describen los conmutadores espaciales, $a^{\alpha}$ y $n_{\alpha \beta}$, resultan

$$
\begin{aligned}
\mathbf{e}_{0}\left(a^{\alpha}\right)= & \left.\frac{1}{3}\left(\Theta \delta_{\beta}^{\alpha}-\frac{3}{2} \sigma_{\beta}^{\alpha}\right)\left(\dot{u}^{\beta}\right)+a^{\beta}\right)+\frac{1}{2} n^{\alpha}{ }_{\beta} \omega^{\beta}-\frac{1}{2} q^{\alpha} \\
\mathbf{e}_{0}\left(n^{\alpha \beta}\right)= & -\frac{1}{3} \Theta n^{\alpha \beta}-\sigma_{\gamma}^{<\alpha} n^{\beta>\gamma}+\frac{1}{2} \sigma^{\alpha \beta} n_{\gamma}^{\gamma}-\left(\dot{u}^{<\alpha}+a^{<\alpha}\right) \omega^{\beta>}-H^{\alpha \beta}+\left(\delta^{\gamma<\alpha} \mathbf{e}_{\gamma}+\dot{u}^{<\alpha}\right)\left(\Omega^{\beta>}\right) \\
& -\frac{2}{3} \delta^{\alpha \beta}\left[2\left(\dot{u}_{\gamma}+a_{\gamma}\right) \omega^{\gamma}-\left(\sigma_{\gamma \delta} n^{\gamma \delta}\right)+\left(\mathbf{e}_{\gamma}+\dot{u}_{\gamma}\right)\left(\Omega^{\gamma}\right)\right] \\
& -\epsilon^{\gamma \delta<\alpha}\left[\left(\dot{u}_{\gamma}+a_{\gamma}\right) \sigma_{\delta}^{\beta>}-\left(\omega_{\gamma}+2 \Omega_{\gamma}\right) n_{\delta}^{\beta>}\right]-\frac{2}{3} \delta^{\alpha \beta}\left(C_{2}\right)+\left(C_{3}\right)^{\alpha \beta}
\end{aligned}
$$

mientras que la evolución de las variables cinemáticas viene dada por

$$
\begin{aligned}
\mathbf{e}_{0}(\Theta)-\mathbf{e}_{\alpha}\left(\dot{u}^{\alpha}\right)= & -\frac{1}{3} \Theta^{2}+\left(\dot{u}_{\alpha}-2 a_{\alpha}\right) \dot{u}^{\alpha}-\left(\sigma_{\alpha \beta} \sigma^{\alpha \beta}\right)+2\left(\omega_{\alpha} \omega^{\alpha}\right) \\
& -\frac{1}{2}(\mu+3 p)+\Lambda \\
\mathbf{e}_{0}\left(\omega^{\alpha}\right)-\frac{1}{2} \epsilon^{\alpha \beta \gamma} \mathbf{e}_{\beta}\left(\dot{u}_{\gamma}\right)= & -\frac{2}{3} \Theta \omega^{\alpha}+\sigma^{\alpha} \beta \omega^{\beta} \\
& -\frac{1}{2} n^{\alpha} \beta \dot{u}^{\beta}-\frac{1}{2} \epsilon^{\alpha \beta \gamma}\left[a_{\beta} \dot{u}_{\gamma}-2 \Omega_{\beta} \omega_{\gamma}\right] \\
\mathbf{e}_{0}\left(\sigma^{\alpha \beta}\right)-\delta^{\gamma<\alpha} \mathbf{e}_{\gamma}\left(\dot{u}^{\beta>}\right)= & -\frac{2}{3} \Theta \sigma^{\alpha \beta}+\left(\dot{u}^{<\alpha}+a^{<\alpha}\right) \dot{u}^{\beta>}-\sigma_{\gamma}^{<\alpha} \sigma^{\beta>\gamma}-\omega^{<\alpha} \omega^{\beta>} \\
& -\left(E^{\alpha \beta}-\frac{1}{2} \pi^{\alpha \beta}\right)+\epsilon^{\gamma \delta<\alpha}\left[2 \Omega_{\gamma} \sigma_{\delta}^{\beta>}-n_{\alpha}^{\beta>} \dot{u}_{\delta}\right]
\end{aligned}
$$

Por último, la evolución de las variables que describen el fluido viene dada por las siguientes ecuaciones

$$
\begin{aligned}
\mathbf{e}_{0}(\mu)+\mathbf{e}_{\alpha}\left(q^{\alpha}\right)= & -\Theta(\mu+p)-2\left(\dot{u}_{\alpha}-a_{\alpha}\right) q^{\alpha}-\left(\sigma_{\alpha \beta} \pi^{\alpha \beta}\right) \\
\mathbf{e}_{0}\left(q^{\alpha}\right)+\delta^{\alpha \beta} \mathbf{e}_{\beta}(p)+\mathbf{e}_{\beta}\left(\pi^{\alpha \beta}\right)= & -\frac{4}{3} \Theta q^{\alpha}-\sigma_{\beta}^{\alpha} q^{\beta}-(\mu+p) \dot{u}^{\alpha}-\left(\dot{u}_{\beta}-3 a_{\beta}\right) \pi^{\alpha \beta} \\
& -\epsilon^{\alpha \beta \gamma}\left[\left(\omega_{\beta}-\Omega_{\beta}\right) q_{\gamma}-n_{\beta \gamma} \pi_{\gamma}^{\delta}\right]
\end{aligned}
$$

El sistema de ecuaciones (A.38)-(A.55) queda completamente determinado si se lo acompaña de una descripción completa del fluido, es decir, si se especifica una ecuación de estado del tipo $p=p(\mu)$, y una ecuación de la evolución para la presión anisótropa, $\pi^{\alpha \beta}$. 


\section{Apéndice B}

\section{Propagación de la luz en modelos de LTB fuera del centro de simetría}

Las ecuaciones diferenciales que describen la trayectoria de los rayos de luz emitidos por una dada fuente, y medidos fuera del centro de simetría, han sido estudiadas mediante dos métodos análogos, presentados en los trabajos [125, 129]. Detallaremos a continuación los lineamientos generales de cada uno de ellos.

\section{B.1. Método I}

Este método fue desarrollado en [125] para estudiar los momentos dipolar y cuadrupolar del fondo cósmico de radiación en modelos de LTB, y fue luego utilizado en [126] para estudiar el problema del horizonte en una familia de modelos de LTB con geodésicas no radiales.

Consideremos un rayo de luz medido en el punto $\mathcal{O}$ a una distancia coordenada $r_{p}$ del centro de simetría. A causa de la simetría esférica de la métrica, el punto $\mathcal{O}$ posee simetría axial respecto de la dirección al centro y, sin pérdida de generalidad, podemos simplificar el problema trabajando las ecuaciones de las geodésicas sobre el plano meridional $\theta=\pi / 2$. La trayectoria de los fotones quedará unívocamente definida por las coordenadas $\left(t_{p}, r_{p}\right)$ del punto $\mathcal{O}$, y el ángulo $\alpha$ comprendido entre la dirección de llegada del fotón y la dirección hacia el centro de simetría. El subíndice $p$ será utilizado, en adelante, para indicar cantidades evaluadas el la posición del punto $\mathcal{O}$.

Dada la métrica (2.1), definimos el vector de onda de un fotón como $k^{\mu} \equiv \mathrm{d} x^{\mu} / \mathrm{d} \lambda$, siendo $\lambda$ un parámetro afín a lo largo de la geodésica. Sobre el plano meridional, resulta $k^{\theta}=0 \mathrm{y}$, para las componentes restantes, tenemos

$$
\begin{aligned}
& \frac{\mathrm{d} t}{\mathrm{~d} \lambda}=k^{t}, \\
& \frac{\mathrm{d} r}{\mathrm{~d} \lambda}=k^{r}=g^{r r} k_{r}=-\frac{1+2 E}{R^{\prime 2}} k_{r}, \\
& \frac{\mathrm{d} \varphi}{\mathrm{d} \lambda}=k^{\varphi}=g^{\varphi \varphi} k_{\varphi}=-\frac{k_{\varphi}}{R^{2}} .
\end{aligned}
$$

A partir de la ecuación de la geodésica,

$$
\frac{\mathrm{d}^{2} x^{\mu}}{\mathrm{d} \lambda^{2}}+\Gamma_{\alpha \beta}^{t} \frac{\mathrm{d} x^{\alpha}}{\mathrm{d} \lambda} \frac{\mathrm{d} x^{\beta}}{\mathrm{d} \lambda}=0
$$


podemos escribir, para cada componente,

$$
\begin{aligned}
\frac{\mathrm{d} k^{t}}{\mathrm{~d} \lambda} & =-\frac{\dot{R}^{\prime}}{R^{\prime 3}}(1+2 E)\left(k_{r}\right)^{2}-\frac{\dot{R}}{R^{3}}\left(k_{\varphi}\right)^{2}, \\
\frac{\mathrm{d} k_{r}}{\mathrm{~d} \lambda} & =\left[\frac{E^{\prime}}{R^{\prime 2}}-(1+2 E) \frac{R^{\prime \prime}}{R^{\prime 3}}\right]\left(k_{r}\right)^{2}-\frac{R^{\prime}}{R^{3}}\left(k_{\varphi}\right)^{2}, \\
\frac{\mathrm{d} k_{\varphi}}{\mathrm{d} \lambda} & =\text { cte. }
\end{aligned}
$$

en donde los símbolos ' $\mathrm{y}^{\prime}$ indican, respectivamente, derivadas con respecto a las coordenadas $t$ y $r$. Además, de la condición $\mathrm{d} s^{2}=0$ para los fotones, resulta

$$
\left(k^{t}\right)^{2}=\frac{1+2 E}{R^{\prime 2}}\left(k_{r}\right)^{2}+\frac{1}{R^{2}}\left(k_{\varphi}\right)^{2} .
$$

La integración de las ecuaciones diferenciales (B.1)-(B.3) y (B.5)-(B.6) se realiza desde el punto $\mathcal{O}$ hasta la posición de la fuente. Las condiciones iniciales se establecen en $\left(t_{p}, r_{p}\right)$ eligiendo

$$
\left\{\begin{aligned}
\lambda_{o} & =\lambda_{p}=0, \\
t_{o} & =t_{p}, \\
r_{o} & =r_{p}, \\
k_{o}^{t} & =\left(k^{t}\right)_{p}=1 .
\end{aligned}\right.
$$

De esta forma, la posición de la fuente queda parametrizada con un valor negativo de $\lambda$.

Teniendo en cuenta que $\alpha$ es el ángulo de incidencia de los fotones respecto a la dirección del centro de simetría, podemos escribir

$$
\begin{aligned}
& \left(k_{r}\right)_{p}=a \cos \alpha, \\
& \left(k_{\phi}\right)_{p}=b \operatorname{sen} \alpha,
\end{aligned}
$$

con $a$ y $b$ dos constantes a determinar. Reemplazando (B.10) y (B.11) en la ecuación (B.8), evaluada esta última en el punto $\mathcal{O}$, tenemos

$$
1=\left(\frac{1+2 E_{p}}{\left(R^{\prime 2}\right)_{p}}\right) a^{2} \cos ^{2} \alpha+\left(\frac{1}{R^{2}}\right) b^{2} \operatorname{sen}^{2} \alpha .
$$

De la expresión anterior, se deduce que para aquellos fotones que inciden en el punto $\mathcal{O}$ resultan

$$
\begin{aligned}
a & =\frac{\sqrt{1+2 E_{p}}}{\left(R^{\prime}\right)_{p}}, \\
b & =\mp \frac{1}{R_{p}},
\end{aligned}
$$

en donde los signos '- ' y ' +' en $b$ corresponden, respectivamente, a fotones con ángulos de incidencia $\alpha \in(0, \pi)$ y $\alpha \in(0, \pi)$. Luego, utilizado la ecuación (B.7), se obtiene

$$
k_{\varphi}=\left(k_{\varphi}\right)_{p}= \pm(R)_{p} \operatorname{sen} \alpha,
$$

y reemplazando $k_{\varphi}$ en la expresión (B.8), encontramos

$$
k_{r}= \pm\left[\left(k^{t}\right)^{2}-\frac{(R)_{p}^{2} \operatorname{sen}^{2} \alpha}{R^{2}}\right]^{1 / 2} \frac{R^{\prime}}{\sqrt{1+2 E}} .
$$


Aquí, los signos '+' y '-' corresponden, respectivamente, a ángulos de incidencia $\alpha \in(-\pi / 2, \pi / 2)$ y $\alpha \in(\pi / 2,3 / 2 \pi)$.

Con el análisis anterior, la trayectoria de los fotones queda determinada completamente por el sistema de ecuaciones diferenciales acopladas dado por [125]

$$
\begin{aligned}
\frac{\mathrm{d} t}{\mathrm{~d} \lambda} & =k^{t} \\
\frac{\mathrm{d} r}{\mathrm{~d} \lambda} & = \pm \frac{\sqrt{1+2 E}}{R^{\prime}}\left[\left(k^{t}\right)^{2}-\frac{(R)_{p}^{2} \operatorname{sen}^{2} \alpha}{R^{2}}\right]^{1 / 2}, \\
\frac{\mathrm{d} k^{t}}{\mathrm{~d} \lambda} & =-\frac{\dot{R}^{\prime}}{R^{\prime}}\left(k^{t}\right)^{2}+\frac{(R)_{p}^{2} \operatorname{sen}^{2} \alpha}{R^{2}}\left[\frac{\dot{R}^{\prime}}{R^{\prime}}-\frac{\dot{R}}{R}\right] .
\end{aligned}
$$

Finalmente, el redshift de una fuente medida en el punto $\mathcal{O}$ con coordenadas $\left(t_{p}, r_{p}\right)$ está definido como [181]

$$
z \equiv k^{t} /\left(k^{t}\right)_{p}-1
$$

en donde $k^{t}$ y $\left(k^{t}\right)_{p}$ son, respectivamente, las componentes espaciales del vector de onda del fotón en la posición de la fuente y en el punto $\mathcal{O}$. Con las condiciones iniciales elegidas, la expresión anterior se reduce a

$$
1+z=k^{t}
$$

\section{B.2. Método II}

Este método fue desarrollado en [129], y utilizado luego para estudiar restricciones impuestas por las anisotropías del fondo cósmico de radiación sobre los modelos de LTB fuera del centro de simetría [130]. Lo presentaremos aquí siguiendo las simplificaciones de [109].

$\mathrm{Al}$ igual que en el método I, el problema presenta simetría axial respecto de la dirección que une el centro de simetría con la posición del punto $\mathcal{O}$, en donde el fotón es medido. Sin pérdida de generalidad, esta dirección es elegida como el eje $z$ del sistema de referencia de la métrica. En este sistema, las coordenadas espaciales del punto $\mathcal{O}$ son $r=r_{0}$ y $\theta=0$. Por ser $\mathcal{O}$ un punto polar, el ángulo acimutal de su posición, $\phi$, resulta degenerado. La trayectoria de los fotones que llegan hasta $\mathcal{O}$ en el tiempo $t=t_{0}$ queda determinada, para todo instante de tiempo, por las coordenadas

$$
\begin{aligned}
t & =t \\
r & =\hat{r}\left(t, t_{0}, r_{0}, \xi\right) \\
\theta & =\hat{\theta}\left(t, t_{0}, r_{0}, \xi\right) \\
\varphi & =\text { cte }
\end{aligned}
$$

en donde $\hat{r}$ y $\hat{\theta}$ son las soluciones de las ecuaciones de la geodésica nula en función de la coordenada temporal $t$, del ángulo de incidencia de los fotones, $\xi$, y de las coordenadas del punto $\mathcal{O}$ en el instante de medición $\left(t_{0}, r_{0}\right)$. Debido a la simetría axial del problema, la trayectoria de los fotones es independiente del ángulo acimutal $\varphi$. Las ecuaciones de las geodésicas resultan 
entonces

$$
\begin{aligned}
\frac{\mathrm{d}^{2} t}{\mathrm{~d} \lambda^{2}}+\frac{\dot{\alpha}}{\alpha}\left(\frac{\mathrm{d} t}{\mathrm{~d} \lambda}\right)^{2}+2 \frac{\alpha^{\prime}}{\alpha} \frac{\mathrm{d} t}{\mathrm{~d} \lambda} \frac{\mathrm{d} r}{\mathrm{~d} \lambda}+ & \\
+\frac{R^{\prime}\left[\dot{R}^{\prime}(1+2 E)-R^{\prime} \dot{E}\right]}{\alpha^{2}(1+2 E)^{2}}\left(\frac{\mathrm{d} r}{\mathrm{~d} \lambda}\right)^{2}+\frac{R \dot{R}}{\alpha^{2}}\left(\frac{\mathrm{d} \theta}{\mathrm{d} \lambda}\right)^{2} & =0, \\
\frac{\mathrm{d}^{2} r}{\mathrm{~d} \lambda^{2}}+\frac{(1+2 E) \alpha \alpha^{\prime}}{R^{\prime 2}}\left(\frac{\mathrm{d} t}{\mathrm{~d} \lambda}\right)^{2}+2 \frac{\dot{R}^{\prime}(1+2 E)-R_{r} \dot{E}}{R^{\prime}(1+2 E)} \frac{\mathrm{d} t}{\mathrm{~d} \lambda} \frac{\mathrm{d} r}{\mathrm{~d} \lambda}+ & \\
+\frac{R^{\prime \prime}(1+2 E)-R^{\prime} E^{\prime}}{R^{\prime}(1+2 E)^{2}}\left(\frac{\mathrm{d} r}{\mathrm{~d} \lambda}\right)^{2}+\frac{R(1+2 E)}{R^{\prime}}\left(\frac{\mathrm{d} \theta}{\mathrm{d} \lambda}\right)^{2} & =0, \\
\frac{\mathrm{d}^{2} \theta}{\mathrm{d} \lambda^{2}}+2 \frac{\dot{R}}{R} \frac{\mathrm{d} t}{\lambda} \frac{\mathrm{d} \theta}{\mathrm{d} \lambda}+2 \frac{R^{\prime}}{R} \frac{\mathrm{d} r}{\mathrm{~d} \lambda} \frac{\mathrm{d} \theta}{\mathrm{d} \lambda} & =0 .
\end{aligned}
$$

Definiendo el momento angular de fotón como

$$
J \equiv R^{2} \frac{\mathrm{d} \theta}{\mathrm{d} \lambda}
$$

la expresión (B.28) puede reescribirse como una ecuación de conservación para el momento angular de la forma

$$
\frac{\mathrm{d}}{\mathrm{d} \lambda} J=0 .
$$

Por otra parte, teniendo en cuenta que la 4-velocidad de los fotones satisface la condición $k^{\mu} k_{\mu}=0$, tenemos que

$$
-\left(\frac{\mathrm{d} t}{\mathrm{~d} \lambda}\right)^{2}+\frac{R^{\prime 2}}{1+2 E}\left(\frac{\mathrm{d} r}{\mathrm{~d} \lambda}\right)^{2}+R^{2}\left(\frac{\mathrm{d} \theta}{\mathrm{d} \lambda}\right)^{2}=0 .
$$

Definamos las cantidades auxiliares $q \equiv \mathrm{d} t / \mathrm{d} \lambda$ y $p \equiv \mathrm{d} r / \mathrm{d} \lambda$. Las condiciones iniciales para la parametrización se eligen de forma tal que $\lambda=0$ en el instante $t=t_{0}, q_{0} \equiv q(\lambda=0)=-1$, $p_{0} \equiv p(\lambda=0)$ y $J_{0} \equiv J(\lambda=0)$.

Para expresar $p_{0}$ y $J_{0}$ en función de cantidades conocidas (al igual que en el método I) tendremos en cuenta el siguiente análisis. Las componentes del vector espacial unitario en la dirección del eje $z$ (determinada esta por el centro de simetría y el punto $\mathcal{O}$ ) son

$$
\vec{v}=\frac{\sqrt{1+2 E_{p}}}{\left(R^{\prime}\right)_{p}}(1,0,0) .
$$

Asimismo, las componentes del vector espacial unitario en la dirección de incidencia del fotón en el instante $t=t_{0}$ son

$$
\vec{u}_{0}=\left|\left(\frac{\mathrm{d} \lambda}{\mathrm{d} t}\right)_{0}\right|\left(\left(\frac{\mathrm{d} r}{\mathrm{~d} \lambda}\right)_{0},\left(\frac{\mathrm{d} \theta}{\mathrm{d} \lambda}\right)_{0},\left(\frac{\mathrm{d} \varphi}{\mathrm{d} \lambda}\right)_{0}\right)=-\frac{1}{q_{0}}\left(p_{0}, \frac{J_{0}}{\left(R^{2}\right)_{0}}, 0\right),
$$

en donde el signo negativo de $u_{0}$ indica que el parámetro $\lambda$ decrece con el tiempo, es decir, que la integración de las ecuaciones se realiza desde el punto $\mathcal{O}$ hasta la fuente. Con estas 


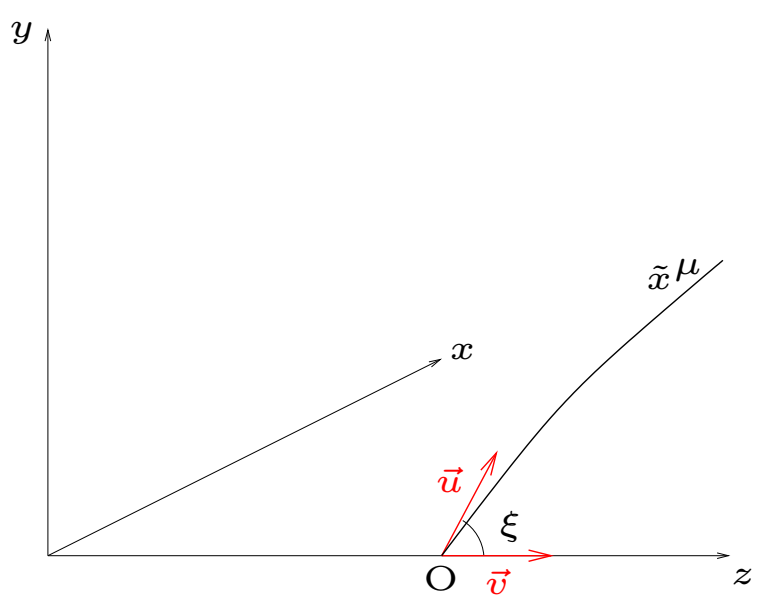

Figura B.1: (extraída de [129]) El fotón incide en el punto $\mathcal{O}$ con un ángulo $\xi$, determinado unívocamente por los vectores espaciales $\vec{u}$ y $\vec{v}$.

definiciones, el ángulo de incidencia de los fotones, $\xi$, queda expresado por el producto de $\vec{v}$ y $\vec{u}$ de la siguiente forma (figura B.1) [129]

$$
\cos \xi=g_{i j} v^{i} u^{j}=-\frac{(R, r)_{p}}{\sqrt{1+2 E_{p}}} \frac{p_{0}}{q_{0}} .
$$

Podemos reescribir entonces

$$
\begin{aligned}
& p_{0}=\frac{\sqrt{1+2 E_{p}}}{\left(R^{\prime}\right)_{p}} \cos \xi, \\
& J_{0}=J=R_{p} \operatorname{sen} \xi
\end{aligned}
$$

en donde hemos tenido en cuenta, para la última expresión, la ecuación de conservación (B.30) para el momento angular.

Concluido este análisis, las ecuaciones (B.26), (B.27) y (B.28) se pueden reescribir en función de las variables $q, p$ y $J$. La integración de este sistema de tres ecuaciones acopladas se resolverá con las condiciones iniciales dadas, y bajo la restricción impuesta por la ecuación (B.31), a saber

$$
-q^{2}+\frac{R^{\prime 2}}{1+2 E} p^{2}+\frac{J^{2}}{R^{2}}=0 .
$$

Calculemos, por último, el redshift de los fotones medidos en función del ángulo de incidencia $\xi$ en forma análoga a como lo hicimos para el caso de geodésicas radiales. Consideremos dos fotones emitidos por una dada fuente en los instantes $t_{1}(\lambda)=t(\lambda)$ y $t_{2}(\lambda)=t(\lambda)+\tau(\lambda)$. La condición (B.31), para cada uno de ellos, se escribe como

$$
\begin{gathered}
\left(\frac{\mathrm{d} t}{\mathrm{~d} \lambda}\right)^{2}=\frac{\left[R^{\prime}(t, r)\right]^{2}}{1+2 E}\left(\frac{\mathrm{d} r}{\mathrm{~d} \lambda}\right)^{2}+R^{2}(t, r)\left(\frac{\mathrm{d} \theta}{\mathrm{d} \lambda}\right)^{2} \\
\left(\frac{\mathrm{d}(t+\tau)}{\mathrm{d} \lambda}\right)^{2}=\frac{\left[R^{\prime}(t+\tau, r)\right]^{2}}{1+2 E}\left(\frac{\mathrm{d} r}{\mathrm{~d} \lambda}\right)^{2}+R^{2}(t+\tau, r)\left(\frac{\mathrm{d} \theta}{\mathrm{d} \lambda}\right)^{2} .
\end{gathered}
$$


Desarrollando la ecuación (B.39) hasta primer orden en $\tau$, resulta

$$
\frac{\mathrm{d} t}{\mathrm{~d} \lambda} \frac{\mathrm{d} \tau}{\mathrm{d} \lambda}=\tau(\lambda)\left[\frac{R^{\prime} \dot{R}^{\prime}}{1+2 E}\left(\frac{\mathrm{d} r}{\mathrm{~d} \lambda}\right)^{2}+R \dot{R}\left(\frac{\mathrm{d} \theta}{\mathrm{d} \lambda}\right)^{2}\right] .
$$

El redshift de la fuente medido en el punto $\mathcal{O}$ se define como

$$
z(\lambda) \equiv \frac{\tau\left(\lambda_{0}\right)}{\tau(\lambda)}-1,
$$

en donde $\lambda_{0}$ indica el valor del parámetro afín en la posición del observador. Diferenciando esta expresión respecto de $\lambda$ obtenemos

$$
\frac{\mathrm{d} z}{\mathrm{~d} \lambda}=-\frac{1}{\tau(\lambda)} \frac{\mathrm{d} \tau(\lambda)}{\mathrm{d} \lambda} \frac{\tau\left(\lambda_{0}\right)}{\tau(\lambda)},
$$

y, teniendo en cuenta la ecuaciones (B.40) y (B.41), resulta

$$
\frac{\mathrm{d} z}{\mathrm{~d} \lambda}=-(1+z) \frac{\mathrm{d} \lambda}{\mathrm{d} t}\left[\frac{R^{\prime} \dot{R}^{\prime}}{1+2 E}\left(\frac{\mathrm{d} r}{\mathrm{~d} \lambda}\right)^{2}+R \dot{R}\left(\frac{\mathrm{d} \theta}{\mathrm{d} \lambda}\right)^{2}\right],
$$

o bien

$$
\frac{\mathrm{d} \ln (1+z)}{\mathrm{d} \lambda}=-\frac{1}{q}\left[\frac{R^{\prime} \dot{R}^{\prime}}{1+2 E} p^{2}+\frac{\dot{R}}{R^{3}} J^{2}\right] .
$$

Esta es la fórmula que expresa el redshift de la fuente en términos de las funciones $q, p$ y $J$. La integración se realiza con la condición inicial $z(\lambda=0) \equiv z_{0}=0$, una vez resuelto el sistema acoplado de ecuaciones diferenciales (B.26)-(B.28). 


\section{Apéndice $\mathrm{C}$}

\section{Otras soluciones de las ecuaciones de Einstein de interés cosmológico}

\section{C.1. La solución de Bianchi tipo I}

Las soluciones exactas de Bianchi describen geometrías espacialmente homogéneas, pero anisótropas. En particular, nos limitaremos a presentar aquí una subclase de estas, conocidas como soluciones de Bianchi tipo I. La descripción que sigue está dada en el formalismo covariante descripto en el apéndice A. Una introducción más detallada puede encontrarse en $[5,185]$.

Las soluciones de Bianchi Tipo I describen las geometrías anisótropas en expansión más simples de la Relatividad General. El elemento de línea que describe a la métrica, en coordenadas comóviles al fluido cosmológico de materia con velocidad $u^{a}=\delta_{0}^{a}$, viene dado por [186]

$$
\mathrm{d} s^{2}=-\mathrm{d} t^{2}+a_{1}^{2}(t) \mathrm{d} x^{2}+a_{2}^{2}(t) \mathrm{d} y^{2}+a_{3}^{2}(t) \mathrm{d} z^{2} .
$$

Es decir, es la generalización homogénea más simple de la geometría espacialmente plana de FLRW, para el caso de diferentes factores de expansión en las tres direcciones espaciales ortogonales. En general, los tres factores de expansión $a_{i}(t)$ son independientes y el factor de expansión promedio queda definido entonces como

$$
S(t) \equiv\left(a_{1} a_{2} a_{3}\right)^{1 / 3} .
$$

Las hipersuperficies con $t=$ cte son planas (esto es, para $t=t_{0}$ todos los coeficientes métricos son constantes). El fluido cosmológico con densidad $\rho$ y presión $p$, que evoluciona ortogonal a dichas superficies, sigue necesariamente un movimiento geodésico y con rotación nula. Estas restricciones quedan bien determinadas si se satisfacen las siguientes relaciones [5]

$$
0=\dot{u}^{\alpha}=\omega^{\alpha}, \quad 0=\tilde{\nabla}_{\alpha} \rho=\tilde{\nabla}_{\alpha} \Theta=\tilde{\nabla}_{\alpha} p, \quad 0={ }^{3} R_{\alpha \beta} .
$$

Las ecuaciones covariantes que describen la evolución de esta geometría son aquellas detalladas en el apéndice A con las restricciones (C.3). Asimismo, los vectores tétrados se definen en términos de las coordenadas $e_{j}{ }^{i}=a^{-1}(t) \delta_{j}{ }^{i}, i, j=1,2,3$. Las cantidades cinemáticas obedecen, por su parte, las siguientes ecuaciones

$$
0=\dot{u}^{\alpha}=\omega^{\alpha}=\Omega^{\alpha}, \quad 0=a^{\alpha}=n_{\alpha \beta}, \quad 0=\mathbf{e}_{\alpha}(\Theta)=e_{\alpha}\left(\sigma_{\beta \gamma}\right), \quad 0=e_{\alpha}(\mu)=e_{\alpha}(p) .
$$


Por ora parte, el shear obedece la restricción

$$
\left(S^{3} \dot{\sigma_{a b}}\right)=0 \Rightarrow \sigma_{a b}=\frac{\Sigma_{a b}}{S 3}, \quad \operatorname{con}\left(\dot{\Sigma_{a b}}\right)=0,
$$

que implica

$$
\sigma^{2}=\frac{\Sigma^{2}}{S^{6}}, \quad \Sigma^{2}=\frac{1}{2} \Sigma_{a b} \Sigma^{a b}, \quad\left(\dot{\Sigma^{2}}\right)=0 .
$$

La ecuación de Friedmann-Lemaître generalizada resulta

$$
3 \frac{\dot{S}^{2}}{S^{2}}=\frac{\Sigma^{2}}{S^{6}}+\frac{M}{S^{3 \gamma}},
$$

en donde $\gamma$ es una constante presente en la ecuación de estado del fluido, dada por $p=(\gamma-1) \mu$ $(\gamma=1 \rightarrow$ polvo y $\gamma=4 / 3 \rightarrow$ radiación).

Las escalas de longitud individuales en cada dirección son [5]

$$
a_{i}(t)=S(t) \exp \left(\Sigma_{i} W(t)\right), \quad i=1,2,3,
$$

con

$$
W(t)=\int \frac{\mathrm{d} t}{S^{3}(t)},
$$

y las constantes $\Sigma_{\alpha}$ satisfacen los vínculos

$$
\Sigma_{1}+\Sigma_{2}+\Sigma_{3}=0, \quad \Sigma_{1}^{2}+\Sigma_{2}^{2}+\Sigma_{3}^{2}=2 \Sigma^{2} .
$$

Las relaciones (C.10) se satisfacen eligiendo

$$
\Sigma_{i}=\frac{2}{3} a \Sigma \operatorname{sen} \alpha_{i}, \quad \alpha_{1}=\alpha, \quad \alpha_{2}=\alpha+\frac{2 \pi}{3}, \quad \alpha_{3}=\alpha+\frac{4 \pi}{3}, \quad \alpha=\text { cte } .
$$

Como ejemplo, consideremos el caso de un fluido sin presión $(\gamma=1)$. Integrando las ecuaciones (C.7) y (C.9) obtenemos, respectivamente,

$$
S(t)=\left(\frac{9}{2} M t^{2}+\sqrt{3} \Sigma t\right)^{1 / 3}, \quad \text { y } \quad W(t)=\frac{1}{\sqrt{3} \Sigma} \ln \left(\frac{t}{\frac{3}{4} M t+\sqrt{3} \Sigma}\right) .
$$

Luego,

$$
a_{i}(t)=S(t)\left(\frac{t^{2}}{S^{3}(t)}\right)^{\frac{2}{3} \operatorname{sen} \alpha_{i}}, \quad i=1,2,3 .
$$

El caso general es anisótropo, y la isotropía se recupera cuando $\Sigma=0$.

Una solución con simetría axial surge en el caso en el que el factor de escala se diferencia sólo en una de las direcciones, es decir, $a_{1}(t)=a_{2}(t) \neq a_{3}(t)$. El modelo de Bianchi tipo I con simetría axial [185] cobra especial interés porque puede ser comparado directamente con un modelo de LTB con el punto de referencia $\mathcal{O}$ ubicado fuera del centro de simetría. A pesar de que ambos modelos poseen simetría axial, existen sin embargo algunas diferencias en la descripción de ambas geometrías. Los modelos de Bianchi tipo I poseen un plano adicional de simetría, normal al eje de simetría del problema (en el caso de LTB fuera del centro, el perfil no homogéneo de densidad elimina esta simetría). ${ }^{1}$

\footnotetext{
${ }^{1}$ Esta diferencia podría llegar a verse cualitativamente en algunos observables cosmológicos utilizados como test de isotropía, como por ejemplo la paralaje cósmica (ver sección 3.1).
} 
Fijando $a_{1}=a_{2}=a(t)$ y $a_{3}(t)=b(t)$ en la expresión (C.1), las ecuaciones de Einstein resultan [185]

$$
\begin{aligned}
H_{a}^{2}+2 H_{a} H_{b} & =8 \pi G_{\rho}, \\
2 \dot{H}_{a}+3 H_{a}^{2} & =-8 \pi G p_{z}, \\
\dot{H}_{a}+H_{a}^{2}+\dot{H}_{b}+H_{b}^{2}+H_{a} H_{b} & =-8 \pi G p_{x},
\end{aligned}
$$

en donde el símbolo 'denota ahora derivadas con respecto al tiempo $t, p_{x}=p_{y}$ y $p_{z}$ son las presiones anisótropas en las correspondientes direcciones, y los parámetros de Hubble se definen como $H_{a} \equiv \dot{a} / a$ y $H_{b} \equiv \dot{b} / b$. La conservación del tensor energía-momento se escribe

$$
\dot{\rho}=-2 H_{a}\left(\rho+p_{x}\right)-H_{b}\left(\rho+p_{z}\right) .
$$

\section{C.2. La solución de Stephani}

La solución general de Stephani [135] no tiene simetrías definidas, y describe una fuente de fluido perfecto con gradiente de presión no nulo. Si la solución presenta además simetría esférica, suele considerarse comúnmente como la solución análoga a la de LTB (esta última, sin embargo, con gradiente de densidad no nulo). Al igual que para el caso de LTB, la solución de FLRW puede recuperarse en el caso límite de presión homogénea.

Las propiedades de la geometría global de la solución general de Stephani fueron estudiadas detalladamente en $[108,279,280,280]$. El elemento de línea que caracteriza a la solución de Stephani, expresado en coordenadas cartesianas, viene dado por [280]

$$
\mathrm{d} s^{2}=D^{2} \mathrm{~d} t^{2}-\frac{R^{2}(t)}{V^{2}}\left(\mathrm{~d} x^{2}+\mathrm{d} y^{2}+\mathrm{d} z^{2}\right),
$$

en donde

$$
\begin{aligned}
V & =1+\frac{1}{4} k(t)\left[\left(x-x_{0}(t)\right)^{2}+\left(y-y_{0}(t)\right)^{2}+\left(z-z_{0}(t)\right)^{2}\right], \\
D & =F(t)\left(\frac{\dot{V}}{V}-\frac{\dot{r}}{R}\right)=F \frac{R}{V} \frac{\partial}{\partial t}\left(\frac{V}{R}\right), \\
k & =\left[C^{2}(t)-\frac{1}{F^{2}(t)}\right] r^{2}(t),
\end{aligned}
$$

con $C, F, R, x_{0}, y_{0}$ y $z_{0}$ funciones arbitrarias del tiempo. La métrica (C.18) satisface las ecuaciones de Einstein con un contenido de materia caracterizado por un fluido perfecto con densidad y presión dadas, respectivamente, por

$$
\begin{aligned}
& \kappa \rho(t)=3 C^{2}(t), \\
& \kappa p(t, x, y, z)=-3 C^{2}(t)+2 C(t) \frac{\frac{\mathrm{d} C}{\mathrm{~d} t} \frac{V}{R}}{\frac{\partial}{\partial t}\left(\frac{V}{R}\right)} .
\end{aligned}
$$

Como en la solución de FLRW, las hipersuperficies ortogonales a las líneas de universo presentan curvatura constante. Sin embargo, en este caso el índice de curvatura $k$ es una 
función del tiempo, que puede cambiar su signo entre una hipersuperficie y otra. En consecuencia, si bien para un dado instante de tiempo el 3-espacio con $t=$ cte tiene una geometría definida, la solución general puede presentar curvatura espacial positiva o negativa en diferentes instantes de tiempo. La existencia de esta solución muestra entonces que, lejos de ser una característica intrínseca que se deriva de la Teoría de la Relatividad General, la distinción entre universos abiertos o cerrados es puramente una consecuencia de las fuertes hipótesis de simetría (en especial, la de homogeneidad) que se imponen a priori para construir diferentes modelos cosmológicos. ${ }^{2}$

Cinemáticamente, la solución está caracterizada por un escalar de expansión y un vector de aceleración no nulos dados, respectivamente, por las siguientes expresiones

$$
\begin{aligned}
& \Theta=-\frac{3}{F}, \\
& \dot{u}^{0}=0, \quad \dot{u}^{i}=\left(\frac{V^{2}}{D R^{2}}\right) D_{, i}, \quad i=1,2,3 .
\end{aligned}
$$

Es decir, que las trayectorias que siguen las partículas necesariamente dejan de ser geodésicas. El shear y la rotación, por su parte, resultan nulos. Asimismo, la métrica es conformemente plana, es decir, que su tensor de Weyl es idénticamente cero [280].

El límite homogéneo se recupera localmente si y sólo si las siguientes condiciones se satisfacen:

1. $k=$ cte, $x_{0}=$ cte, $y_{0}=$ cte, $z_{0}=$ cte ;

2. $\dot{u}^{\alpha}=0 ; \mathrm{y}$

3. $p=f(\rho)$, en donde $f$ es una función independiente de $x, y, z$.

Como mencionamos al comienzo de esta sección, la solución de Stephani no posee simetrías definidas. Es en el caso con $x_{0}, y_{0}, \mathrm{y} z_{0}$ constantes en el que surge la simetría esférica respecto a la línea $x=x_{0}, y=y_{0} \mathrm{y} z=z_{0}$ (notemos, sin embargo, que aún en este caso la métrica resulta no homogénea).

La solución de Stephani con simetría esférica es presentada comúnmente como solución complementaria a la de LTB, pues describe un fluido con densidad uniforme pero presión no homogénea. Para analizar la familia de soluciones de Stephani con simetría esférica, es conveniente trabajar en coordenadas esféricas. El elemento de línea en coordenadas isótropas ${ }^{3}$ resulta $[136]$

$$
\mathrm{d} s^{2}=-\frac{a^{2}}{\dot{a}}\left[\frac{\left(\frac{\dot{V}}{a}\right)}{\left(\frac{V}{a}\right)}\right]^{2} \mathrm{~d} t^{2}+\frac{a^{2}}{V^{2}}\left(\mathrm{~d} r^{2}+r^{2} \mathrm{~d} \Omega^{2}\right),
$$

\footnotetext{
${ }^{2}$ Una distinción interesante entre las hipótesis de homogeneidad y "homogeneidad débil" puede encontrarse en la referencia [280].

${ }^{3}$ Las coordenadas isótropas $(t, r, \theta, \phi)$, al igual que en el caso de las solución homogénea de FLRW, se relacionan con las coordenadas no isótropas $(t, \bar{r}, \theta, \phi)$ por las siguientes expresiones [281]

$$
\begin{aligned}
& \bar{r}=\frac{r}{1+\frac{1}{4} k r^{2}}=\frac{r}{V(r)}, \\
& r=\frac{2 \bar{r}}{1+\sqrt{1-k \bar{r}^{2}}} .
\end{aligned}
$$


en donde

$$
V(t, r)=1+\frac{1}{4} k(t) r^{2}
$$

y el símbolo ' indica $\partial / \partial t$. La función $V(t, r)$ con $k=0, \pm 1$ recupera la forma de los modelos de Friedmann-Lemaître en las mismas coordenadas [282]. La función $a(t)$ juega el papel de factor de escala generalizado y $k(t)$ es el llamado índice de curvatura.

El tensor de energía-momento del fluido perfecto es $T^{a b}=[\rho(t)+p(t, r)] u^{a} u^{b}+p(t, r) g^{a b}$, en donde la densidad de energía y la presión se escriben en las nuevas coordenadas como

$$
\begin{aligned}
\rho(t) & =3\left[\frac{\dot{a}^{2}(t)}{a^{2}(t)}+\frac{k(t)}{a^{2}(t)}\right], \\
p(t, r) & =\left[-1+\frac{1}{3} \frac{\dot{\rho}(t)}{\rho(t)} \frac{\left[\frac{V(t, r)}{a(t)}\right]}{\left[\frac{V(t, r)}{a(t)}\right]}\right] \rho(t) \equiv w_{e f}(t, r) \rho(t),
\end{aligned}
$$

con $w_{e f}(t, r)$ el índice barotrópico efectivo.

\section{C.2.1. Modelos cosmológicos de Stephani}

Un estudio detallado de los modelos cosmológicos basados en la solución general de Stephani puede encontrarse en [87]. En particular, dos familias de modelos cosmológicos con soluciones de Stephani exactas y esféricamente simétricas fueron presentados en [136, 283]. La primera corresponde a la elección $(\ddot{V / a})=0$, mientras que la segunda satisface la condición $(k / a)=0$ (usualmente llamados modelos tipo I y II, respectivamente).

El ajuste de los datos provenientes de las SN tipo Ia para estas familias de modelos, realizado con la motivación de poder describir las observaciones astronómicas sin necesidad de recurrir a una componente de energía oscura en el universo, fue presentado primeramente en [284]. ${ }^{4}$ Asimismo, algunos de los modelos tipo I fueron cotejados con datos observacionales en $[285,286]$.

Los modelos tipo II resultan más simples. El elemento de línea que los describe se reduce a $[176,283]$

$$
\mathrm{d} s^{2}=-\frac{1}{V^{2}} \mathrm{~d} t^{2}+\frac{a^{2}}{V^{2}}\left(\mathrm{~d} r^{2}+r^{2} \mathrm{~d} \Omega^{2}\right) .
$$

Dos subtipos de estos modelos (propuestos en [287] y [136, 284, 288], respectivamente) fueron estudiados recientemente en la referencia [282]. El primero está caracterizado en el centro de simetría por un fluido con ecuación de estado barotrópica, mientras que el segundo admite una ecuación de estado no barotrópica, pero con expresión analítica. La ventaja de trabajar con este último es que la evolución del factor de escala se conoce en forma exacta, y coincide con aquella correspondiente a los modelos de Friedmann-Lemaître con contenido de polvo. Los perfiles no homogéneos de presión representados por estos dos tipos de modelos tienen características diferentes, y dan lugar a un análisis amplio para estas familias de modelos de Stephani. Los autores de la referencia [282] presentan la expresión para la distancia de luminosidad calculada para estos modelos, y encuentran restricciones sobre observaciones fuera del centro de simetría, sobre la ecuación de estado del fluido cosmológico y sobre los gradientes de presión no homogéneos, a partir del catalogo Union 2 de SN tipo Ia [50].

\footnotetext{
${ }^{4}$ Este fue, de hecho, el primer trabajo que propuso a los modelos no homogéneos como alternativa viable para explicar las observaciones de SN tipo Ia que indicaban una expansión acelerada del universo.
} 
Es importante mencionar que al trabajar con modelos cosmológicos basados en la solución de Stephani, se está suponiendo implícitamente la existencia de un fluido con ecuación de estado variable en hipersuperficies con $r=$ cte. La interpretación usual de fluidos perfectos excluye, en general, ecuaciones de estado barotrópicas (porque la densidad del fluido resulta homogénea, pero su presión no), a pesar de que las mismas proveen un esquema termodinámico estricto [289] y pueden ser interpretadas físicamente [290]. Sin embargo, los elementos de fluido pueden presentar, individualmente, características exóticas, como presión negativa [87]. Por esta razón, algunos trabajos en la literatura [291] han desestimado la viabilidad de la solución de Stephani para describir el universo. Sin embargo, algunas de estas características inusuales están también presentes en la descripción de la propia energía oscura en el marco del modelo cosmológico estándar. Es entonces absolutamente válido llevar a cabo un estudio serio de estas soluciones, como un primer paso en la exploración de modelos cosmológicos más complejos [87]. 


\section{Apéndice D}

\section{El Redshift drift: un potencial observable para distinguir cosmologías}

Los resultados presentados en el capítulo 3 para el cálculo del redshift drift son válidos si las fuentes son cercanas al punto de medición. Para enmarcar estos resultados en un escenario más general, presentaremos en este apéndice una breve revisión de los trabajos que calculan el redshift drift en diferentes geometrías para fuentes con un $z$ arbitrario.

Como mencionamos anteriormente, este cálculo es en general complejo y requiere la utilización de herramientas numéricas para integrar las geodésicas nulas que determinan la trayectoria de los fotones.

\section{El redshift drift en modelos de FLRW}

La predicción del la variación del redshift cosmológico para diferentes modelos isótropos y homogéneos ha sido estudiada por varios autores [167, 168, 169, 170, 171, 172, 292].

Para el caso de algunos de los modelos viables de energía oscura, compatibles con diversos conjuntos de datos observacionales, la estimación del redshift drift fue presentada en [292]. Algunos de los resultados obtenidos por estos autores se muestran en la figura D.1. En el gráfico de la izquierda se observan las curvas obtenidas para diferentes valores de los parámetros cosmológicos del modelo $\Lambda \mathrm{CDM}$. El gráfico de la derecha muestra, por su parte, las predicciones teóricas para diferentes modelos de energía oscura. En todos los casos, el redshift drift resulta positivo para fuentes cercanas (en acuerdo con los resultados obtenidos en esta tesis a partir de las aproximaciones trabajadas en el capítulo 3).

\section{El redshift drift en modelos no homogéneos}

En el marco de los modelos no homogéneos con simetría esférica, el redshift drift ha sido estudiado en [166] para ser utilizado como test del Principio Copernicano.

El caso particular de los modelos de LTB con perfiles de void y observaciones realizadas en el centro de simetría fue estudiada en [88]. La curva obtenida, y su comparación con la predicción para otros modelos cosmológicos, se muestran en la figura D.2. En todos los modelos de void estudiados, la curva del redshift drift es negativa para todo $z$, al igual que para aquellos modelos de FLRW con constante cosmológica nula y con dinámica dominada por 

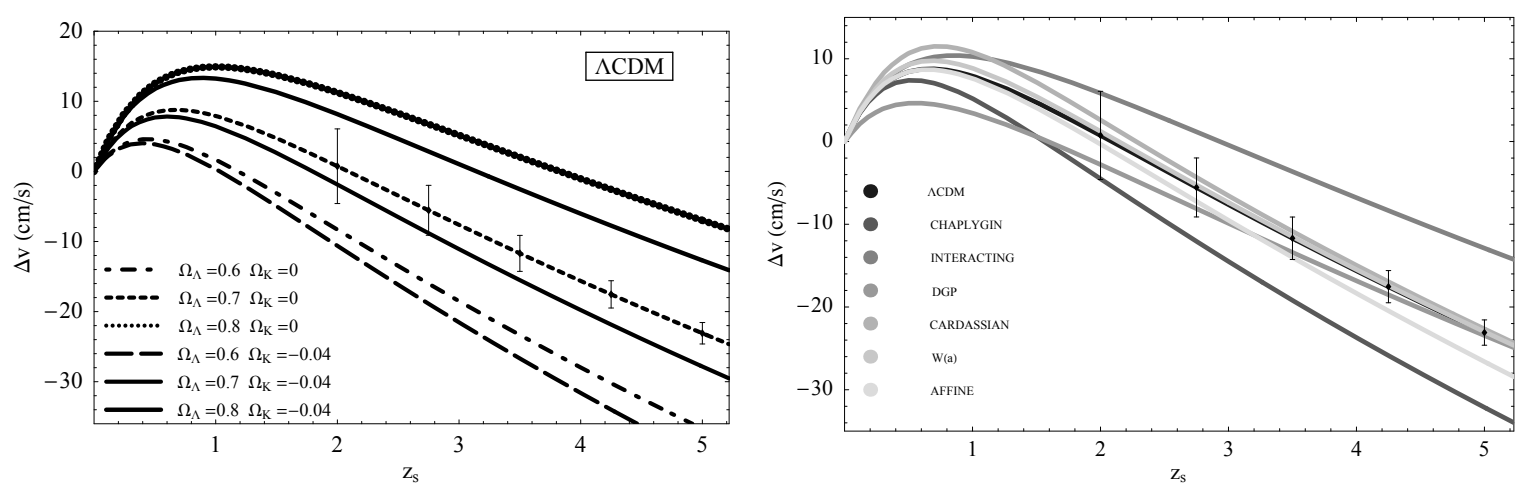

Figura D.1: (extraída de [292]) Predicción del corrimiento espectroscópico en la velocidad de objetos extragalácticos para el modelo $\Lambda \mathrm{CDM}$ (izquierda) y para diferentes modelos de energía oscura (derecha). En todos los casos, se observa que el valor estimado para el redshift drift resulta positivo para fuentes con $z \lesssim 1,5$.

materia oscura. Comparaciones similares para modelos de LTB han sido estudiadas también en los trabajos [173, 180].

La predicción del redshift drift en modelos de Stephani fue estudiada en [176, 177, 178]. Los resultados de estos trabajos muestran que, al menos para observaciones realizadas en el centro de simetría, existe una subclase de los modelos de Stephani para la cual el valor estimado para el redshift drift es cualitativamente diferente de aquel calculado para los modelos de LTB, y cuantitativamente diferente del estimado para el modelo $\Lambda$ CDM (figura D.3). Para el caso de perfiles con regiones no homogéneas poco pronunciadas $\left(\Omega_{i n h}=0,40\right)$ la curva del redshift drift se aproxima a aquella calculada para los modelos de LTB, mientras que para regiones no homogéneas grandes el comportamiento sigue la tendencia del modelo $\Lambda$ CDM. Para regiones no homogéneas muy grandes, el redshift drift resulta positivo para $z$ cada vez mayores, y en el límite en el que el perfil no homogéneo es totalmente dominante $\left(\Omega_{i n h}=1\right)$ la dependencia de $\dot{z}$ con $z$ resulta lineal y positiva para todo $z\left(d z / d t=H_{0} z / 2\right)$. Nuevamente, observamos que el redshift drift puede ser utilizado como un test observacional para distinguir los modelos de Stephani de otros modelos cosmológicos.

Casos con menos simetrías, como aquellos modelos basados en la solución de Székeres, fueron estudiados en $[175,183,184,293]$. Los resultados para el caso de modelos tipo "queso suizo" se muestran en la figura D.4. Al igual que para los modelos de void de LTB, la predicción del redshift drift indica un valor negativo para todo $z$. Sin embargo, ambos modelos podrían ser distinguibles por la significativa diferencia esperada para fuentes con $z \sim 2$. 


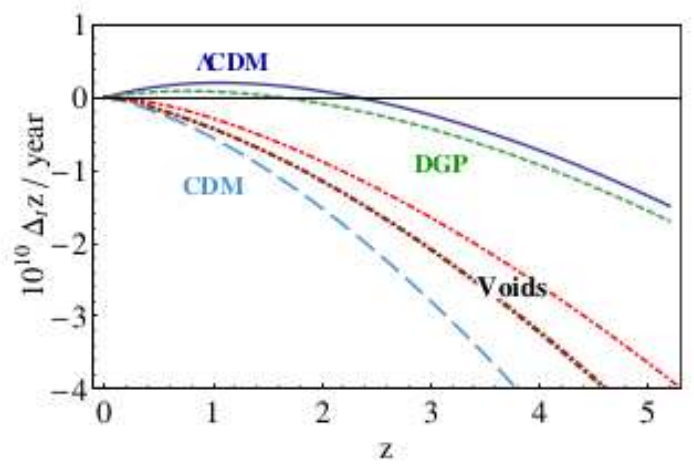

Figura D.2: Predicción para el redshift drfit anual para modelos de void de LTB y su comparación con otros modelos basados en la solución de FLRW. La curva correspondiente a los modelos de void es siempre negativa, y se ubica entre la correspondiente al modelo $\Lambda$ CDM y aquella predicha para un universo dominado sólo por materia oscura (CDM) (figura extraída de [88]).

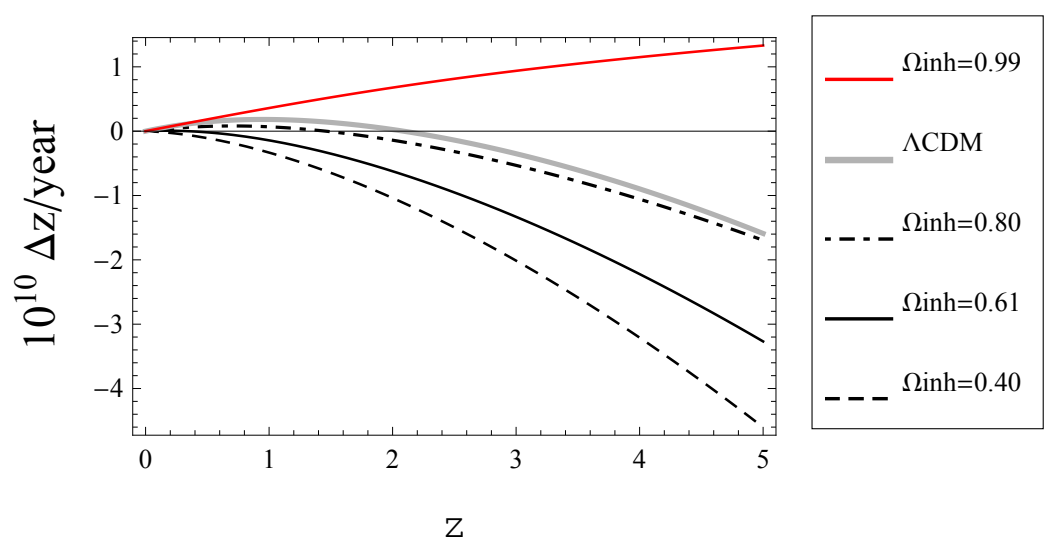

Figura D.3: Estimación del redshift drift como función del redshift cosmológico para los modelos de Stephani (figura extraída de [176]). El parámetro $\Omega_{i n h}$ caracteriza el tamaño de la región no homogénea. Para apartamientos pequeños de la homogeneidad $\left(\Omega_{i n h} \sim 0,4\right)$ la curva del redshift drift se mantienen siempre negativa, como en los modelos de LTB, mientras que para regiones no homogéneas mayores el comportamiento esperado para $\dot{z}$ imita a aquel estimado para el modelo $\Lambda$ CDM. 


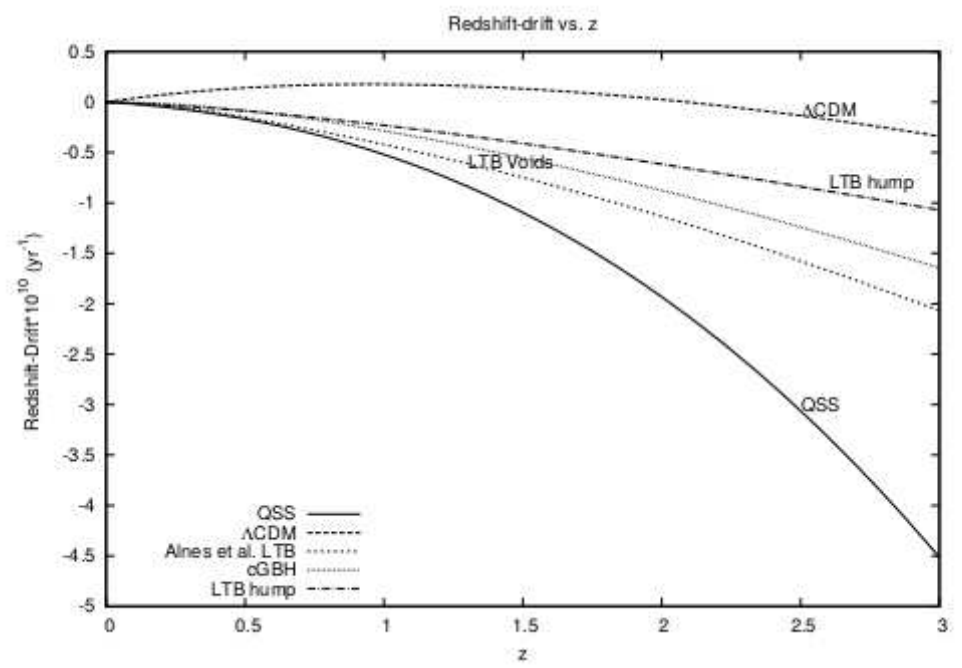

Figura D.4: (extraída de [183]) Estimación del redshift drift como función del redshift cosmológico para modelos de "queso suizo" construidos a partir de la solución cuasiesférica de Székeres [176] . 


\section{Apéndice E}

\section{El redshift drift cosmográfico en teorías $f(R)$}

Como mencionamos en la sección 3.3, el tratamiento cosmográfico de cantidades observables puede utilizarse para comprarar aproximaciones cinemáticas con aquellas calculadas a partir de la dinámica de un dado modelo cosmológico. En esta dirección, hemos desarrollado un método basado en la comparación de cantidades observables estimadas a partir de sus tratamientos cosmográfico y dinámico. Dicha comparación se realiza utilizando desarrollos en series de potencias del redshift, y el método puede ser aplicado, en principio, a cualquier observable cosmológico que pueda ser escrito en términos del parámetro de Hubble, $H(z)$.

Una aplicación interesante de este método de comparación se da para aquellos modelos cosmológicos desarrollados en el marco de las teorías de gravedad modificada $f(R)$, y basados también en la métrica de FLRW. En particular, el tratamiento cinemático del reshift drift puede ser utilizado en estos casos para obtener cotas sobre los parámetros libres que presentan estos modelos.

Desarrollaremos en este apéndice dicho método de comparación y su aplicación directa al estudio de dos modelos cosmológicos $f(R)$. Este estudio fue abordado en forma complementaria al trabajo desarrollado en esta tesis. Comenzaremos por hacer una breve introducción sobre las teorías de gravedad modificada $f(R)$, para pasar luego a la aplicación del tratamiento cosmográfico del redshift drift en el marco de dichas teorías.

\section{E.1. Breve introducción a las teorías $f(R)$}

La predicciones teóricas de la Relatividad General han sido confirmadas por numerosas observaciones y experimentos. Sin embargo, la exploración de teorías alternativas que puedan apartarse de la Relatividad General en los límites de curvatura débil o fuerte ha crecido a lo largo del último siglo de manera significativa [11, 12, 13].

Las teorías de gravedad modificada están basadas en correcciones y/o extensiones de la Teoría de la Relatividad General a través de la incorporación de invariantes de curvatura de orden superior (o de acoplamientos de campos escalares) en la acción Hilbert-Einstein del campo gravitacional [11]. En particular, las llamadas teorías $f(R)$ se construyen a partir de una generalización de la densidad lagrangiana usual, que reemplaza el escalar de curvatura $R$ 
por una función de este. Así, la acción usual de Hilbert-Einstein se modifica de la forma

$$
\mathcal{S}=\int \mathrm{d}^{4} x \sqrt{-g} f(R),
$$

en donde $g$ es el determinante de una métrica $g_{\mu \nu}$, y $f$ es una función arbitraria del escalar de curvatura que debe satisfacer ciertas restricciones teóricas y observacionales [11, 12, 13].

Las teorías $f(R)$ pueden clasificarse en tres tipos, según el rol que se le atribuya a la conexión afín. En cada uno de ellos, la ecuación de conservación para el tensor energíamomento es válida, y la materia y la gravedad están acopladas mínimamente [18]. Daremos a continuación una breve reseña de cada uno de estos tipos [294].

\section{Formalismo métrico}

En la versión métrica de las teorías $f(R)$, la acción dada por la expresión

$$
S=\frac{1}{2 \kappa} \int \sqrt{-g} f(R) \mathrm{d} x^{4}+S_{M}\left(g_{\mu \nu}, \psi\right)
$$

se varía con respecto a la métrica $g_{\mu \nu}$. El término $S_{M}$ representa la acción de la materia, que resulta independiente de la conexión afín. Las ecuaciones de movimiento resultantes son de cuarto orden en las derivadas del tensor métrico:

$$
\frac{\mathrm{d} f(R)}{\mathrm{d} R} R_{\mu \nu}-\frac{1}{2} f(R) g_{\mu \nu}-\left[\nabla_{\mu} \nabla_{\nu}-g_{\mu \nu} \square\right] \frac{\mathrm{d} f(R)}{\mathrm{d} R}=\kappa T_{\mu \nu}
$$

en donde $T_{\mu \nu}$ es el tensor de energía-momento del campo de materia, definido por

$$
T_{\mu \nu}=-\frac{2}{\sqrt{-g}} \frac{\delta S_{M}}{\delta g^{\mu \nu}},
$$

y la derivada covariante es definida usando la conexión usual de Levi-Civita. Tomando la traza de la ecuación (E.3) resulta la relación

$$
\frac{\mathrm{d} f(R)}{\mathrm{d} R} R=-2 f(R)+3 \square \frac{\mathrm{d} f(R)}{\mathrm{d} R}=\kappa T,
$$

que es comparable a $R=-\kappa T$ en el caso de Relatividad General (para el cual $f(R)=R$ ).

\section{Formalismo de Palatini}

En este segundo tipo de teorías $f(R)$ la métrica y la conexión son consideradas campos independientes, y la acción de la materia, $S_{M}$, es independiente de la conexión. La acción se escribe entonces como

$$
S=\frac{1}{2 \kappa} \int \sqrt{-g} f(\mathcal{R}) \mathrm{d} x^{4}+S_{M}\left(g_{\mu \nu}, \psi\right),
$$

con $\mathcal{R}=g^{\mu \nu} \mathcal{R}_{\mu \nu}$, y en donde el correspondiente tensor de Riemann es construido considerando, a priori, la conexión afín $\Gamma$ independiente de la métrica.

De la variación de la acción con respecto a la métrica y la conexión afín, resulta [12]

$$
\begin{aligned}
f^{\prime}(\mathcal{R}) \mathcal{R}_{(\mu \nu)}-\frac{1}{2} f(\mathcal{R}) g_{\mu \nu} & =\kappa T_{\mu \nu} \\
\bar{\nabla}_{\lambda}\left(\sqrt{-g} f^{\prime}(\mathcal{R}) g^{\mu \nu}\right) & =0
\end{aligned}
$$


en donde el símbolo ' indica derivadas con respecto a $\mathcal{R}$ y la derivada $\bar{\nabla}$ es construida con la conexión $\Gamma$. La Teoría de la Relatividad General se recupera en el caso $f(\mathcal{R})=\mathcal{R}$. Tomando la traza de la ecuación (E.7), resulta

$$
f^{\prime}(\mathcal{R}) \mathcal{R}-2 f(\mathcal{R})=\kappa T,
$$

es decir, que en este caso la relación entre $\mathcal{R}$ y $T$ es algebraica (no hay presentes modos escalares).

Por otra parte, de la ecuación (E.7) se tiene [12]

$$
\Gamma_{\mu \nu}^{\lambda}=\frac{1}{f^{\prime}(\mathcal{R})} g^{\lambda \sigma}\left[\nabla_{\mu}\left(f^{\prime}(\mathcal{R}) g_{\mu \sigma}\right)-\nabla_{\sigma}\left(f^{\prime}\left(\mathcal{R} g_{\mu \nu}\right)\right]\right.
$$

Dado que esta ecuación relaciona $\Gamma$ con $\mathcal{R}$ y con la métrica, y que a su vez $\mathcal{R}$ y $T$ son en principio intercambiables haciendo uso de la ecuación algebraica (E.9), entonces la conexión puede ser expresada en términos del campo de materia y la métrica, es decir, resulta un campo auxiliar. Otra forma de ver esto es reescribir la ecuación (E.7) como

$$
G_{\mu \nu}=\frac{\kappa}{f^{\prime}} T_{\mu \nu}-\frac{1}{2} g_{\mu \nu}\left(\mathcal{R}-\frac{f}{f^{\prime}}\right)+\frac{1}{f^{\prime}}\left(\nabla_{\mu} \nabla_{\nu}-g_{\mu \nu} \square\right) f^{\prime}-\frac{3}{2} \frac{1}{f^{\prime 2}}\left[\left(\nabla_{\mu} f^{\prime}\right)\left(\nabla_{\nu} f^{\prime}\right)-\frac{1}{2} g_{\mu \nu}\left(\nabla f^{\prime}\right)^{2}\right],
$$

en donde el tensor de Einstein y las derivadas covariantes están construidas ahora a partir de la conexión de Levi-Civita, y $\mathcal{R}$ está expresado en términos de $T$ usando la ecuación (E.9). De esta forma, puede interpretarse el formalismo de Palatini como una forma análoga a la Relatividad General, pero con una fuente del campo gravitacional modificada. Una de las características más importantes es, sin embargo, es que esta modificación involucra derivadas de tercer orden en el campo de materia. ${ }^{1}$

\section{Formalismo de métrica afín}

En este último caso, la acción de la materia depende también de la conexión afín, que es en principio independiente de la métrica. Esto es, una acción de la forma [12, 296]

$$
S=\frac{1}{2 \kappa} \int \sqrt{-g} f(\mathcal{R}) \mathrm{d}^{4} x+S_{M}\left(g_{\mu \nu}, \Gamma_{\mu \nu}^{\lambda}, \psi\right) .
$$

\section{Aplicación a problemas cosmológicos y astrofísicos}

Distintos modelos cosmológicos basados en teorías de gravedad modificada $f(R)$ han sido construidos para satisfacer los experimentos actuales en el Sistema Solar y en los laboratorios terrestres [80, 82, 297, 298], así como también para ajustar satisfactoriamente observaciones astronómicas de diferente naturaleza.

En el régimen de curvatura débil, una de las principales motivaciones que impulsaron el estudio de estas teorías es la evidencia de una expansión acelerada del universo cuando las observaciones astronómicas son interpretadas en el marco del modelo cosmológico estándar. En este contexto, la familia de teorías $f(R)$ que reproducen una expansión acelerada del

\footnotetext{
${ }^{1}$ En relación a este punto, algunas consecuencias interesantes pueden observarse en la estructura interna de estrella de neutrones, como fue estudiado en uno de los trabajos complementarios a esta tesis [295].
} 
universo es muy amplia. Por ello, es importante estudiar cómo se puede acotar el espacio de parámetros libres que las caracterizan a través de observaciones astronómicas. Distintas restricciones pueden obtenerse a partir de datos cosmológicos y astrofísicos, así como también imponiendo que la teoría describa correctamente las fases de desaceleración y aceleración en la evolución del universo [299]. En este contexto, mostraremos en la siguiente sección una manera de restringir el espacio de parámetros de modelos cosmológicos $f(R)$ a partir de la aproximación cosmográfica del redshift drift desarrollada en la sección 3.3. Los resultados de este trabajo fueron publicados en un artículo de circulación internacional [192] y forman parte del aporte original desarrollado en el trabajo de esta tesis. ${ }^{2}$

En el régimen de curvatura fuerte, por el contrario, las evidencias observacionales que podrían testear la Teoría de Relatividad General son escasas. Los escenarios astrofísicos que involucran agujeros negros o estrellas de neutrones pueden ser excelentes laboratorios para testear desviaciones a la relatividad general en el marco de teorías alternativas. En esta dirección, hemos investigado en forma paralela al trabajo presentado en esta tesis la estructura interna de estrellas de neutrones en el marco de teorías $f(R)$, tanto para el formalismo métrico como para el formalismo de Palatini, en el régimen de campo gravitacional fuerte. Se estudió la estructura interna de estos objetos compactos, así como también sus configuraciones masa-radio. Los resultados de nuestros trabajos fueron publicados en dos artículos de circulación internacional [295, 301], que forman parte del trabajo de investigación original que se desarrollo en forma complementaria a esta tesis.

\section{E.2. Tratamiento cosmográfico para teorías $f(R)$}

Como una aplicación directa del tratamiento cosmográfico del redshift drift presentado en la sección 3.3, hemos ampliado nuestro trabajo al análisis de teorías $f(R)$ a partir de dicho observable. En particular, estudiamos en este apéndice cómo puede vincularse la curva observacional del redshift drift cosmográfico con su predicción teórica calculada a partir de la dinámica de las teorías $f(R)$. De esta comparación pueden obtenerse cotas sobre algunos parámetros libres de las funciones $f$. Ejemplificamos esta aplicación con dos modelos particulares presentados en [80] y [302], para los cuales encontramos restricciones que acotan el espacio de parámetros significativamente [192].

Dada una teoría $f(R)$, las ecuaciones del campo gravitacional que resultan de variar $\mathcal{S}$ con respecto a la métrica son [303]

$$
\begin{array}{r}
f^{\prime} R-2 f^{\prime \prime}\left(\ddot{R}+\frac{3 \dot{a} \dot{R}}{a}\right)+3 f^{\prime \prime \prime} \dot{R}^{2}+\kappa T=0, \\
f^{\prime} \ddot{R}+\frac{1}{2} f-3 f^{\prime \prime} \frac{\dot{a} \dot{R}}{a}+\kappa \ddot{T}=0,
\end{array}
$$

donde $\mathrm{y}^{\prime}$ denotan, respectivamente, derivadas con respecto a $R$ y $t$. Además, se satisfacen las relaciones $\ddot{R}=3 \dot{a} / a$ y $R=-6\left(\frac{\ddot{a}}{a}+\frac{\dot{a}^{2}}{a^{2}}\right)$, y hemos supuesto un universo plano.

\footnotetext{
${ }^{2}$ Un criterio basado en datos observacionales e independiente del desarrollado en este apéndice fue presentado en [300]. El mismo se basa en las condiciones de energía que debe satisfacer el contenido de materia en el universo, y posibilita decidir si una dada teoría $f(R)$ es apropiada para describir el universo sin necesidad de resolver las ecuaciones de movimiento para el campo cosmológico, ni hacer transformaciones de frame o suposiciones adicionales sobre el modelo.
} 
A partir de las ecuaciones (E.13) y (E.14), la densidad de energía y la presión del fluido cosmológico pueden expresarse en términos de $f$ y sus derivadas de la forma

$$
\begin{aligned}
& \rho=-f^{\prime} \ddot{R}-\frac{f}{2}+3 f^{\prime \prime} \frac{\dot{a} \dot{R}}{a}, \\
& p=-\frac{f^{\prime}}{3}(\ddot{R}+R)+\frac{f}{2}-f^{\prime \prime}\left(\ddot{R}-\frac{2 \dot{a} \dot{R}}{a}\right)-f^{\prime \prime \prime} \dot{R}^{2} .
\end{aligned}
$$

Las ecuaciones (E.13 y (E.14) nos permiten escribir [304]:

$$
H=\frac{1}{6 \dot{R} f^{\prime \prime}}\left(6 H^{2} f^{\prime}-2 \rho_{m}-f+R f^{\prime}\right),
$$

con $\rho_{m}=\rho_{m, 0} a^{-3}=3 H_{0}^{2} \Omega_{m, 0} a^{-3}$. Teniendo en cuenta la ecuación (3.78), el redshift drift puede expresarse en términos de $H(z)$ de la forma

$$
\frac{\Delta z}{\Delta t_{0}}=(1+z) H_{0}-H(z)
$$

Luego, resulta

$$
\frac{\Delta z}{\Delta t_{0}}(z)=\frac{\dot{a}_{0}-\dot{a}(z)}{a(z)}=H_{0}\left\{(z+1)-\frac{1}{6} \frac{f(R)-R f^{\prime}(R)+6 H^{2} f^{\prime}(R)-2 \rho_{m}}{H_{0} \dot{R}(t) f^{\prime \prime}(R)}\right\}
$$

en donde $f, R, H$, y $\rho_{m}$ son funciones de $z \cdot{ }^{3}$ Aplicando la regla de la cadena, $\frac{\mathrm{d} f}{\mathrm{~d} z}=\frac{\mathrm{d} f}{\mathrm{~d} R} \mathrm{~d} R \frac{\mathrm{d} t}{\mathrm{~d} t} \mathrm{~d} z$ y expresiones análogas para las otras funciones de $z$, encontramos a primer orden

$$
\begin{aligned}
\frac{\Delta z}{\Delta t_{0}}(z)=\left\{H_{0}\right. & +\frac{1}{6 H_{0} f_{0}^{\prime \prime}}\left[\frac{1}{\dot{R}_{0}}\left(f_{0}^{\prime \prime} \dot{R}_{0}\left(R_{0}-6 H_{0}^{2}\right)+2 \dot{\rho}_{m, 0}-12 H_{0} \dot{H}_{0} f_{0}^{\prime}\right)\right. \\
& \left.\left.+\left(\frac{\ddot{R}_{0}}{\dot{R}_{0}^{2}}+\frac{f_{0}^{\prime \prime \prime}}{f_{0}^{\prime \prime}}\right)\left(f_{0}+f_{0}^{\prime}\left(6 H_{0}^{2}-R_{0}\right)-2 \rho_{m, 0}\right)\right]\right\} z+\mathcal{O}\left(z^{2}\right) .
\end{aligned}
$$

Por último, utilizando las relaciones $R_{0}=6\left(\dot{H}_{0}+2 H_{0}^{2}\right), \dot{H}_{0}=-H_{0}^{2}\left(1+q_{0}\right)$ y $\ddot{H}_{0}=H_{0}^{3}\left(j_{0}+\right.$ $\left.3 q_{0}+2\right)$, junto con las definiciones de los parámetros cinemáticos (3.68)-(3.71), obtenemos

$$
\begin{aligned}
\frac{\Delta z}{\Delta t_{0}}(z)=\{[ & \left.6 q_{0} H_{0}^{2} f_{0}^{\prime}+f_{0}-6 \Omega_{m, 0} H_{0}^{2}\right]\left(j_{0}-q_{0}-2\right)^{2} \frac{f_{0}^{\prime \prime \prime}}{6 f_{0}^{\prime \prime}}+ \\
& +H_{0}^{2} f_{0}^{\prime \prime}\left[\left(j_{0}-2\right)^{2}-q_{0}\left(3 q_{0}+\left(q_{0}-j_{0}\right)^{2}-2 j_{0}\right)\right] \\
& +f_{0}^{\prime}\left[q_{0}\left(q_{0}^{2}+6 q_{0}+2 j_{0}+s_{0}\right)+2 j_{0}+4\right]+\frac{f_{0}}{36 H_{0}^{2}}\left(-s_{0}-q_{0}^{2}-6-8 q_{0}\right) \\
& \left.+\frac{\Omega_{m, 0}}{6}\left(s_{0}+3 j_{0}+5 q_{0}+q_{0}^{2}\right)\right\} \frac{z}{f_{0}^{\prime \prime} H_{0}\left(j_{0}-q_{0}-2\right)^{2}}+\mathcal{O}\left(z^{2}\right) .
\end{aligned}
$$

La ecuación (E.21) expresa, para una dada teoría, el redshift drift en términos de $f$ y sus derivadas, evaluadas al tiempo $t_{0}$. Es posible entonces comparar el término lineal en $z$ de los

\footnotetext{
${ }^{3}$ Notemos que la presencia de $f^{\prime \prime}$ en el denominador de esta expresión podría llevar a divergencias, pues la cantidad $f^{\prime \prime}$ es, en general, pequeña si la teoría está construida para imitar una evolución cercana a aquella descripta por el modelo $\Lambda$ CDM. En los ejemplos analizados en este trabajo se ha verificado explícitamente que el producto $H_{0} \dot{R}(t) f^{\prime \prime}(R)$ no cause divergencias indeseables.
} 
desarrollos cinemático y dinámico, dados por las ecuaciones (3.76) y (E.21), respectivamente. Como resultado obtenemos una relación entre $f(R)$, sus derivadas y los parámetros cinemáticos, dada por

$$
\begin{aligned}
& \left\{\left[q_{0}\left(q_{0}\left(q_{0}+6\right)+2 j_{0}+s_{0}\right)+2\left(j_{0}-2\right)\right] f_{0}^{\prime}\right. \\
& \left.\quad+\left[s_{0}+q_{0}\left(q_{0}+8\right)+6\right] f_{0}-\left[q_{0}\left(q_{0}+5\right)+s_{0}+3 j_{0}\right] \Omega_{m, 0}\right\} f_{0}^{\prime \prime}+ \\
& \quad\left[\left(q_{0}-j_{0}\right)^{2}+4\left(1+q_{0}-j_{0}\right)\right]\left\{f_{0} f_{0}^{\prime \prime \prime}+6 H_{0}^{2}\left[\left(q_{0} f_{0}^{\prime}-\Omega_{m, 0}\right) f_{0}^{\prime \prime \prime}+\left(f_{0}^{\prime \prime}\right)^{2}\right]\right\}=0 .
\end{aligned}
$$

Notemos que la restricción de trabajar a primer orden en $z$ no está relacionada con mediciones del redshift drift para fuentes con $z \ll 1$, sino con el hecho de que términos de orden superior involucran derivadas superiores del factor de escala, $\ell_{0}$, de las cuales actualmente no se disponen datos observacionales. Por otra parte, cabe mencionar que la ecuación (E.22) es una condición necesaria para cualquier teoría $f(R)$. Trabajando con órdenes superiores en $z$ de las ecuaciones (3.76) y (E.21), podríamos obtener más condiciones necesarias para la $f(R)$ y sus derivadas (de hecho, en teoría sería un número infinito de estas), que la teoría en cuestión debería satisfacer para describir el redshift drift para todo orden en $z$.

A continuación se detallan dos ejemplos con teorías $f(R)$ particulares, que muestran explícitamente cómo la ecuación (E.22) puede utilizarse para encontrar cotas sobre los parámetros libres de cada modelo.

\section{E.2.1. Ejemplo 1: modelo de Hu \& Sawicki}

El modelo de gravedad $f(R)$ propuesto por Hu \& Sawicki [80] es tal que produce una expansión acelerada del universo, sin introducir una constante cosmológica, e incluye la fenomenología del modelo $\Lambda \mathrm{CDM}$ en el límite de curvatura pequeña. Consideremos la acción de HilbertEinstein modificada, de la forma

$$
S=\int \mathrm{d}^{4} x \sqrt{-g}\left[\frac{f(R)}{\kappa^{2}}+\mathcal{L}_{m}\right],
$$

en donde $R$ es el escalar de Ricci, $\kappa^{2}=8 \pi G$ y $\mathcal{L}_{m}$ la densidad lagrangiana de la materia. El caso $f(R)=R+$ cte equivale a tener a una constante cosmológica. En lo que sigue, trabajaremos en el frame de Jordan.

La forma de la función $f(R)$ se elige de forma tal que el modelo satisfaga determinadas propiedades observacionales. Esto es: (i) que imite la cosmología del modelo $\Lambda$ CDM en el límite de curvatura grande, en donde este último está bien testeado a través de los datos del CMB; (ii) que acelere la expansión del universo a bajos redshifts, con una historia evolutiva cercana a la del modelo $\Lambda \mathrm{CDM}$, pero sin introducir una constante cosmológica verdadera; (iii) que posea suficientes grados de libertad como para abarcar los fenómenos observacionales a bajo redshift actualmente aceptados; y (iv) que incluya el modelo $\Lambda$ CDM como caso límite para producir desviaciones pequeñas en la Teoría de la Relatividad General, que cumplan los tests cosmológicos y locales en el Sistema Solar. Estos requerimientos sugieren un comportamiento de la forma [80]

$$
\begin{aligned}
\lim _{R \rightarrow \infty} f(R) & =R+\text { cte } \\
\lim _{R \rightarrow 0} f(R) & =R
\end{aligned}
$$


que puede ser satisfecho por una clase general de modelos $f(R)$ con una ley de potencias quebrada, de la forma

$$
f(R)=R-m^{2} \frac{c_{1}\left(R / m^{2}\right)^{n}}{c_{2}\left(R / m^{2}\right)^{n}+1}
$$

con $n>0$ y $m^{2}$ definida como

$$
m^{2} \equiv \frac{\kappa^{2} \bar{\rho}_{0}}{3}=(8315 \mathrm{Mpc})^{-2}\left(\frac{\Omega_{m} h^{2}}{0,13}\right)
$$

en donde $\bar{\rho}_{0}=\bar{\rho}(\log a=0)$ es la densidad promedio hoy. Las constantes $c_{1}$ y $c_{2}$ son parámetros adimensionales del modelo. El signo de $f(R)$ es elegido de forma tal que resulte

$$
f^{\prime \prime} \equiv \frac{\mathrm{d}^{2} f(R)}{\mathrm{d} R^{2}}>0
$$

para $R \gg m^{2}$, asegurando así que la solución sea estable en el régimen de densidades altas.

A diferencia de otros modelos, no se introduce una constante cosmológica verdadera en la teoría. Sin embargo, para curvaturas grandes comparadas con la escala de masa $m^{2}, f(R)$ puede expandirse en la forma

$$
\lim _{m^{2} / R \rightarrow 0} f(R) \simeq R-\frac{c_{1}}{c_{2}} m^{2}+\frac{c_{1}}{c_{2}^{2}} m^{2}\left(\frac{m^{2}}{R}\right)^{n} .
$$

resultando una constante cosmológica en el límite $c_{1} / c_{2}^{2} \rightarrow 0$ y $c_{1} / c_{2}$ fijo, para los tests de gravedad locales y cosmológicos. Además, para un valor finito de $c_{1} / c_{2}^{2}$, la curvatura deja de decrecer con la densidad de materia y el modelo da lugar a una aceleración similar a la descripta por el modelo $\Lambda$ CDM.

Para estudiar la historia evolutiva de estos modelos, es conveniente primero acotar el espacio de parámetros de la $f(R)$ de forma tal que la expansión sea compatible con las observaciones, es decir, que imite $\Lambda \mathrm{CDM}$ durante la época de aceleración. Esta condición es equivalente a elegir un valor para el campo hoy tal que sea $f_{0}^{\prime} \equiv f^{\prime}(\ln a=0) \ll 1$ o, equivalentemente, $R_{0} \gg m^{2}$ [80]. Bajo estas condiciones, el desarrollo dado por la ecuación (E.29) vale para toda la historia evolutiva pasada, y la curvatura puede aproximarse de la forma

$$
R=\kappa^{2} \rho-2 f \approx \kappa^{2} \rho+2 \frac{c_{1}}{c_{2}} m^{2},
$$

en donde el término $2 f$ es casi constante e imita la densidad de energía de una constante cosmológica. Para aproximar la historia de la expansión a la del modelo $\Lambda$ CDM, con una constante cosmológica caracterizada por $\tilde{\Omega}_{\Lambda}$ y una densidad de materia $\tilde{\Omega}_{m}$, se considera

$$
\frac{c_{1}}{c_{2}} \approx 6 \frac{\tilde{\Omega}_{\Lambda}}{\tilde{\Omega}_{m}},
$$

dejando de esta manera sólo dos parámetros libres en el modelo, $n$ y $c_{1} / c_{2}^{2}=6 \tilde{\Omega}_{\Lambda} / c_{2} \tilde{\Omega}_{m} \cdot{ }^{4}$

\footnotetext{
${ }^{4}$ Notemos que, como el valor crítico de la densidad y del parámetro de Hubble dependen de $f^{\prime}, \tilde{\Omega}_{m}$ es $\Omega_{m}$ sólo si $\lim _{c_{1} / c_{2}^{2} \rightarrow 0} \tilde{\Omega}_{m}=\Omega_{m}$, mientras que la densidad de materia en unidades físicas no cambia $\left(\tilde{\Omega}_{m} \tilde{H}_{0}^{2}=\Omega_{m} H_{0}^{2}\right)$.
} 
Para una historia evolutiva del tipo $\Lambda$ CDM plano se tiene entonces

$$
\begin{aligned}
& R \approx 3 m^{2}\left(a^{-3}+4 \frac{\tilde{\Omega}_{\Lambda}}{\tilde{\Omega}_{m}}\right) \\
& f^{\prime}=1-n \frac{c_{1}}{c_{2}^{2}}\left(\frac{m^{2}}{R}\right)^{n+1} .
\end{aligned}
$$

La curvatura y el valor del campo hoy resultan

$$
\begin{aligned}
R_{0} & \approx m^{2}\left(\frac{12}{\tilde{\Omega}_{m}}-9\right) \\
f_{0}^{\prime} & \approx 1-n \frac{c_{1}}{c_{2}^{2}}\left(\frac{12}{\tilde{\Omega}_{m}}-9\right)^{-n-1}
\end{aligned}
$$

Dado que los tests cosmológicos y aquellos provenientes del Sistema Solar pueden estudiarse convenientemente a través del valor del campo cosmológico hoy, reescribimos $c_{1} / c_{2}^{2}$ en función de esta cantidad. De esta forma, el modelo queda parametrizado por $n$ y $f_{0}^{\prime}$.

La ecuación (E.35) nos permite expresar $c_{1} / c_{2}^{2}$ en términos de los parámetros $n, f_{0}^{\prime} \mathrm{y}$ $\tilde{\Omega}_{m, 0}$. Asimismo, derivadas de $f$ orden superior pueden ser expresadas en forma análoga en función de las mismas cantidades. Tendremos en cuenta el valor $\tilde{\Omega}_{m, 0}=0,274 \pm 0,007$ [52] y consideraremos $f_{0}^{\prime}$ y $n$ como los únicos parámetros libres de la teoría. Bajo estas condiciones, la ecuación (E.22) resulta

$$
\begin{aligned}
& f_{0}^{\prime}(n)=\frac{1}{A}\{[\left.q_{0}\left(5 q_{0}-6 j_{0}+8+\left(q_{0}-j_{0}\right)^{2}\right)+\left(j_{0}-2\right)^{2}\right] 4 n \\
&+\left[2\left(j_{0}+1\right)+q_{0}\left(q_{0}+6\right)+s_{0}\right] 9 \tilde{\Omega}_{m, 0}^{2} \\
&+\left[j_{0}\left(j_{0}-8\right)-2 q_{0}\left(j_{0}+q_{0}+8\right)-3\left(s_{0}+2\right)\right] 6 \tilde{\Omega}_{m, 0} \\
&\left.\quad+\left[q_{0}\left(2\left(10+3 q_{0}-2 j_{0}\right)+\left(q_{0}-j_{0}\right)^{2}\right)+2\left(s_{0}+6\right)\right] 4\right\},
\end{aligned}
$$

con

$$
\begin{gathered}
A=\left[q_{0}\left(\left(q_{0}-j_{0}\right)^{2}-6 j_{0}+8+5 q_{0}\right)+\left(j_{0}-2\right)^{2}\right] 4 n+\left[s_{0}+q_{0}^{2}+8 q_{0}+6\right] 9 \tilde{\Omega}_{m, 0}^{2} \\
\left.-\left[3 s_{0}+18+22 q_{0}-\left(j_{0}-2\right)^{2}+\left(2 q_{0}+1\right)^{2}\right)\right] 6 \tilde{\Omega}_{m, 0} \\
+4\left[q_{0}\left(2\left(5 q_{0}+16-4 j_{0}\right)+\left(q_{0}-j_{0}\right)^{2}\right)+2\left(s_{0}+10-2 j_{0}\right)\right] .
\end{gathered}
$$

La expresión (E.36) relaciona los parámetros libres del modelo, $f_{0}^{\prime}$ y $n$, con $\tilde{\Omega}_{m}$ y los parámetros cinemáticos $H, q, j$ y $s$, en donde todas las cantidades están evaluadas a tiempo $t=$ hoy. La figura E.1 muestra la relación $f_{0}^{\prime}(n)$ obtenida con los valores $q_{0}=-0,669 \pm 0,052$, $j_{0}=0,284 \pm 0,151$ y $s_{0}=-0,680 \pm 0,456$ [193], y la correspondiente propagación de errores. La curva muestra un comportamiento asintótico hacia el valor $f_{0}^{\prime}=1$, que corresponde al límite de Relatividad General. Se incluye también la cota obtenida a partir de experimentos en el Sistema Solar, dada por $\left|f_{0}^{\prime}-1\right|<0,1$ [80]. Luego, a partir de este útimo límite, observamos que las restricciones provenientes del redshift drift favorecen valores para $n$ mayores que 3 , aproximadamente, en acuerdo con las cotas encontradas anteriormente en [305]. 


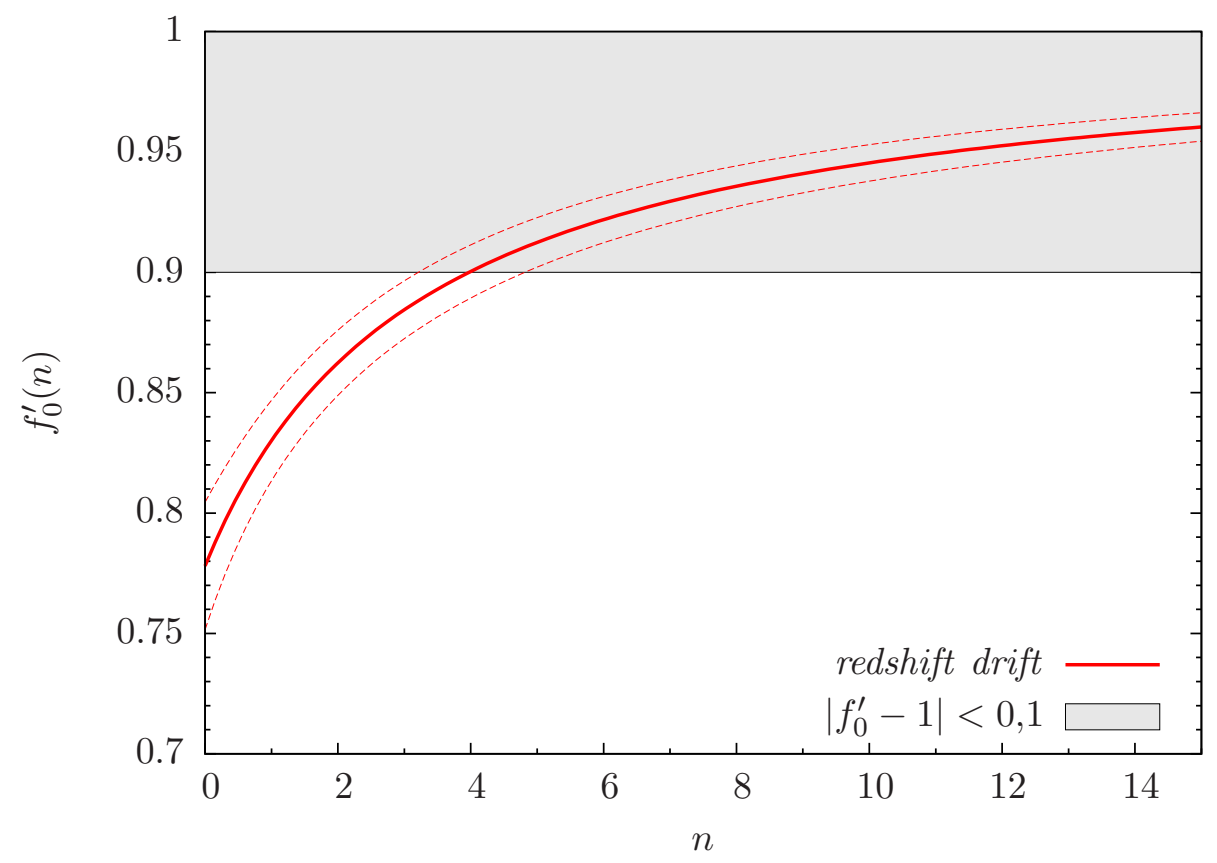

Figura E.1: Límites impuestos en el espacio de parámetros correspondiente al modelo propuesto por Hu y Sawicki (resultados de autoría propia, publicados en [192]). La curva llena corresponde a la relación entre $f_{0}^{\prime}$ y $n$ obtenida a partir de la ecuación (E.22). Las líneas a trazos representan la propagación de los errores en las mediciones de los parámetros cinemáticos y la densidad de materia. La región sombreada indica los valores de $f_{0}^{\prime}$ que están en buen acuerdo con los límites obtenidos a partir de experimentos en el Sistema Solar.

\section{E.2.2. Ejemplo 2: gravedad exponencial}

Analizaremos ahora las restricciones impuestas por la ecuación (E.22) sobre el modelo de gravedad modificada exponencial propuesto en [302]. La función $f(R)$ viene dada en este caso por la expresión

$$
f(R)=R-c r[1-\exp (-R / r)]
$$

en donde $c$ y $r$ son dos parámetros positivos del modelo. Esta $f(R)$ fue específicamente elegida para: (i) prescindir de una constante cosmológica en el modelo (notemos que la función se anula en el límite de curvatura baja); (ii) recuperar Relatividad General en el régimen de curvatura grande; (iii) incorporar una transición de escala (a través del parámetro $r$ ), para ser ajustada a partir de observaciones, en lugar de ser predefinida a partir del valor $R_{0}$; y (iv) recuperar Relatividad General en los regímenes de curvatura grande local, como en el caso del Sistema Solar o de galaxias.

El producto $\mathrm{cr}$ puede escribirse en términos de $\Omega_{m, 0}$ como [302]

$$
c r=6 m^{2}\left(\Omega_{m, 0}^{-1}-1\right) .
$$

La ecuación (E.22) para la forma exponencial de la función $f(R)$ da lugar a una relación 
explícita entre los parámetros adimensionales $c$ y $r / m^{2}$, a saber

$$
\begin{aligned}
c\left(\frac{r}{m^{2}}\right)= & \frac{1}{B}\left\{\left[\left(3+s_{0}+2 j_{0}\left(q_{0}+1\right)-\left(q_{0}-1\right)^{2}\right)\right.\right. \\
& \left.\quad-\left(q_{0}\left(q_{0}+5\right)+s_{0}+q_{0}^{2}+3 j_{0}\right) \Omega_{m, 0}\right] 6 \Omega_{m, 0} \frac{r}{m^{2}} \\
& \left.+\left[4\left(1+q_{0}-j_{0}\right)+\left(q_{0}-j_{0}\right)^{2}\right] 36\left(\Omega_{m, 0}-1\right)\right\} \exp \left(\frac{6\left(1-q_{0}\right)}{\frac{r}{m^{2}} \Omega_{m, 0}}\right),
\end{aligned}
$$

con

$$
\begin{gathered}
B=\left\{[ q _ { 0 } ( q _ { 0 } + 8 ) + 6 + s _ { 0 } ] \Omega _ { m , 0 } ( \frac { r } { m ^ { 2 } } ) \left[\left(\exp \left(\frac{6\left(1-q_{0}\right)}{\frac{r}{m^{2}} \Omega_{m, 0}}\right)-1\right]\right.\right. \\
-6\left[4\left(1+q_{0}-j_{0}\right)+\left(q_{0}-j_{0}\right)^{2}\right] \exp \left(\frac{6\left(1-q_{0}\right)}{\frac{r}{m^{2}} \Omega_{m, 0}}\right) \\
\left.+6\left[q_{0}\left(q_{0}\left(q_{0}+7\right)+4+s_{0}\right)+\left(j_{0}-1\right)^{2}-1\right]\right\} \Omega_{m, 0}\left(\frac{r}{m^{2}}\right) \\
-\left[\left(j_{0}-2\right)^{2}+q_{0}\left(\left(q_{0}-j_{0}\right)^{2}+8-6 j_{0}+5 q_{0}\right)\right] 36 .
\end{gathered}
$$

Esta expresión, junto con la ecuación (E.39), se muestra en la figura E.2, en donde tuvimos en cuenta que la distancia a la superficie de último scattering del fondo cósmico de radiación puede ajustarse a aquella estimada por el modelo $\Lambda \mathrm{CDM}$ con el valor de densidad presente $\Omega_{m, 0}=0,2$ sólo si $c \geq 1,5$ [302]. La intersección de ambas curvas (con sus respectivos errores) se da para valores $c \gtrsim 6$, con lo cual se tiene que $r / m^{2} \lesssim 2,7$. Es importante notar que el rango de los posibles valores que puede tomar el parámetro $c$ calculado a partir del presente método $(c \geq 1,27)$ mejora las cotas antes encontradas en [306].

\section{E.2.3. Discusión}

Diferentes teorías alternativas han sido estudiadas con el propósito de resolver los problemas asociados a la Relatividad General, tanto en el régimen de campo débil como fuerte [307]. En particular, ciertas teorías $f(R)$ de la gravitación son capaces de reproducir una expansión acelerada del universo sin la necesidad de proponer una constante cosmológica ni la existencia de "energía oscura", satisfaciendo con éxito los límites impuestos por observaciones galácticas y del Sistema Solar.

Motivados por este escenario, hemos desarrollado un método para establecer cotas sobre los parámetros libres de teorías $f(R)$. El mismo se basa en la comparación de dos desarrollos en series de potencias de $z$, válidos para cualquier observable cosmológico que pueda ser escrito en términos de $H(z)$. El primer desarrollo es puramente cosmográfico (es decir, que es independiente de la dinámica que domina la evolución del factor de escala y sólo supone una métrica homogénea e isótropa), mientras que el segundo se basa en la dependencia de $H(z)$ con $z$ impuesta por las ecuaciones de campo gravitacional características de las teorías $f(R)$. La comparación de ambos desarrollos da como resultado una relación entre $f$, sus derivadas, y los parámetros cinemáticos (con todas estas cantidades evaluadas a $z=0$ ). Dichas relaciones deben ser satisfechas por cualquier teoría $f(R)$.

En particular, cuando trabajamos con el redshift drift, el método permite estimar cotas de diferentes $f(R)$. Para el caso del modelo propuesto por Hu \& Sawicki [80], encontramos cotas para el parámetro $n$ que están en buen acuerdo con aquellos valores calculados a partir de otros observables. Para el caso de la teoría de gravedad exponencial estudiada en [302], 


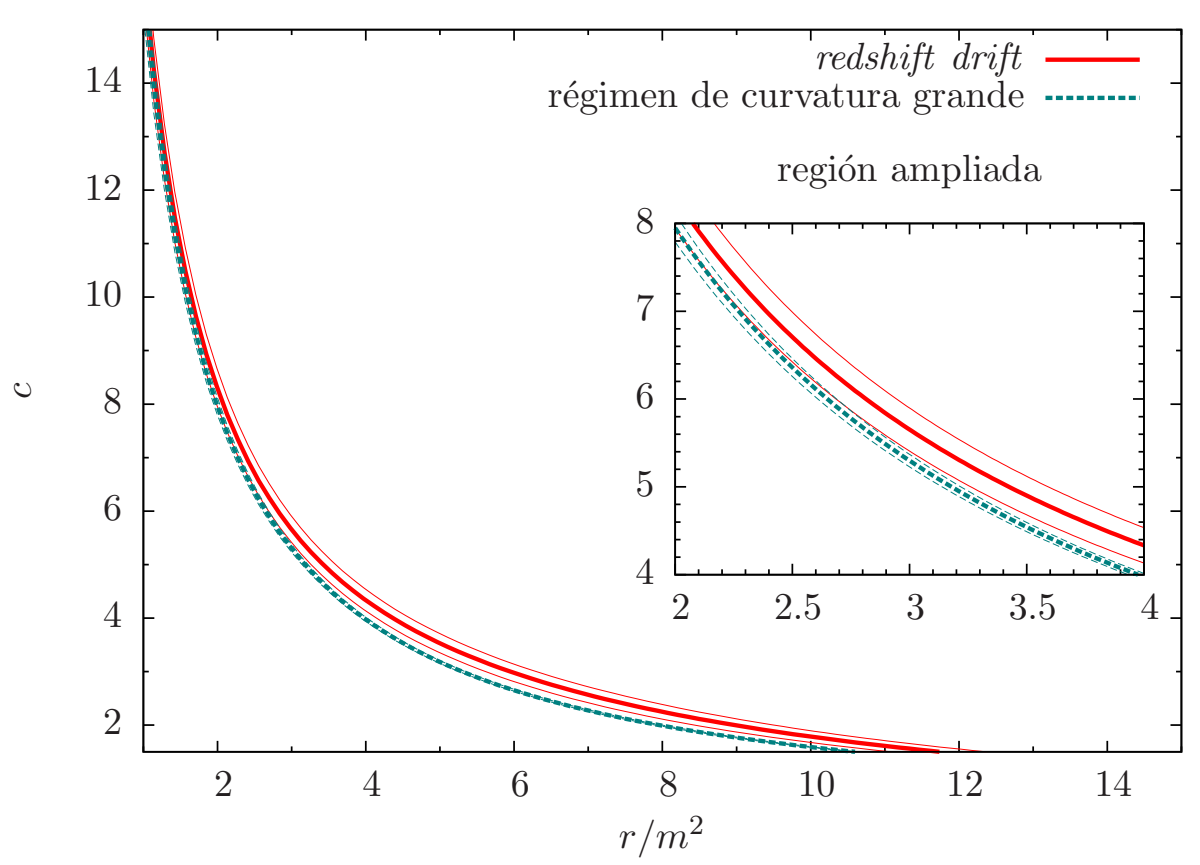

Figura E.2: Restricciones en el espacio de parámetros de la teoría de gravedad exponencial encontradas a partir del tratamiento cosmográfico del redshift drift (líneas continuas), y aquellas dadas por la relación (E.39) entre los parámetros libres de la teoría (líneas de trazos). En ambos casos, las curvas más finas representan la propagación de errores que se arrastra de la estimación de los parámetros cinemáticos y la densidad de materia a tiempo presente (resultados de autoría propia, publicados en [192]).

el límite obtenido para el parámetro $c$ es más fuerte que aquellos previamente obtenidos utilizando otros criterios. Por otra parte, encontramos un límite adicional para el parámetro $r / m^{2}$, que no presentaba cotas previas [192].

Por otra parte, cabe resaltar que el método que desarrollamos no queda restringido sólo a las teorías $f(R)$, sino que puede ser aplicado a cualquier teoría alternativa de la gravitación que respete las hipótesis de homogeneidad e isotropía impuestas por la métrica de FLRW. Asimismo, los límites encontrados a partir del desarrollo cosmográfico del redshift drift pueden complementarse con otros criterios que restringen las $f(R)$, como es el caso de las condiciones de energía [79], con el objetivo de poder decidir si una dada teoría $f(R)$ es viable o no. 


\section{Agradecimientos}

Esta tesis fue desarrollada con el apoyo de las Becas Internas tipo I y II otorgadas por Consejo Nacional de Investigaciones Científicas y Técnicas, y del Programa de Doutorado Cooperativo del Centro Latinoamericano de Física y el International Center for Theoretical Physics.

A nivel académico, quiero agradecer a la Facultad de Ciencias Astronómicas y Geofísicas y al Instituto Argentino de Radioastronomía, por brindarme el lugar de trabajo con las instalaciones adecuadas para desarrollar esta tesis. En particular, quiero reconocer las facilidades brindadas por la Facultad para la utilización del cluster Seminare. Quiero agradecer, asimismo, la hospitalidad brindada en reiteradas ocasiones por la Universidad Estadual do Rio de Janeiro y el Centro Brasileiro de Pesquisas Físicas.

Queiro agradecer a mis colegas platenses, por el intercambio científico y el ambiente de trabajo agradable y solidario. En particular, al Grupo de Astrofísica, Relatividad y Cosmología de la Facultad, especialmente a Susana J. Landau y Claudia G. Scóccola; a mis compañeras de oficina (Noelia Jiménez, Lucila Kraiselburd, Mercedes Mosquera, María Pía Pichirilli y Carolina Negrelli); a Eduardo Suárez, por la administración de Úrsula; y a Favio I. Zyserman, por el asesoramiento para abordar dificultades numéricas. Quiero agradecer también a mis colegas cariocas, por la calidez con la que fui siempre recibida. En particular, a Marcelo Chiapparini, Nelson Pinto Neto, Felipe Tovar Falciano, Patrick Peter, Junior Diniz Toniato, Marcela Campista, Beatriz B. Siffert, y, muy especialmente, a Azucena Rivasplata Paz. Quiero dedicar también un agradecimiento especial a Chris Clarkson, Marco Regis y Woei Chet Lim, por las fructíferas discusiones y la colaboración científica llevada a cabo.

Un cálido abrazo en forma de agradecimiento quiero dedicar a mi hermana Virginia, por la incontable bibliografía que me facilitó durante los años de licenciatura y doctorado.

Asimismo, dedico una una mención especial para mis directores: a Gustavo E. Romero, le agradezco el haberme acompañado y orientado desde mis años de estudiante, siempre con buena predisposición y confianza; y a Santiago E. Perez Bergliaffa quiero agradecerle, muy especialmente, la dedicación, paciencia, solidaridad, confianza y respeto que me ha brindado durante todos los años que dirigió mi doctorado, y destacar la calidad humana que siempre caracterizó nuestro trabajo.

A nivel personal, les agradezco a todas las personas que me acompañaron durante estos cinco años con amor y alegría.

Del lado de allá:

A Felipe Nin, Montse Jiménez, Rhenan Amaral, Nini Carrizo, Josué Viera, a la Mansão Santo Amaro (Elo, Luca, Amanda, Cami, Leo, Gus, Thiago, Walt, Matthew, Toni y Lisa) y, muy especialmente, a Nachito Hamad (que ya es un poco de todos lados).

Del lado de acá:

A mis amigos platenses: Cin Peri, Flor Vieyro, Javito Martí (y a la bella Sofi!®), Tuna Gargiulo, Olguita Bracco, Cris Vega, Mari Guzmán, Juan P. Caso, Lean Sesto, Charly Escudero, Bren Melendez, María Victoria del Valle, Juan P. Calderón, Romi Galván, AlmitaØ, Leo Monachesi, Fede García, Fran de Gerónimo, Santi Perdomo, Andrés Cesanelli, Luis Gómez, José Gordoa, Gabi (namaste!) y a la BuYa Sambareggae (Leito, Tía Costi, Tío Fer, CamilitoO, Juli Toni, Antobella, Palle, Florcita, Lucre, Negra, Juli Dela, Angie Bellatrix, Tino, Osito, Barbie, Lu, Agus, Tiki, Lore, Yami, Ali y Cami). 
Un agradecimiento especial también quiero dedicar al Elenco Estable, que me aguanta el corazón desde hace más de 20 años: Anita Visuara (y Lauto!!®), Dani Bur, Martita Poggio, Vitorita Bianco, Valen Brodsky, Naty Behar Sosa, Colito Matz, Flora Prada, Sabri Gomez, Joha Daquino, Romi Hernández y Yas Whebi.

Por último, y con el corazón en las manos, le agradezco a mi familia incondicional el amor inconmensurable que siempre me ha brindado, y a quien dedico esta tesis: Vir, Pato, Juli, Lina, Fer, Ivita, Ropito, Tía Gra, Tío Oscar, Mati, Anto y, muy especialmente, a Mingo y a Carmen, que me siguen abrazando cada día... 


\section{Bibliografía}

[1] G. F. R. Ellis, Royal Astronomical Society, Quarterly Journal 16, 245 (1975).

[2] Planck Collaboration et al., ArXiv e-prints 1502.01582 (2015).

[3] Z. Chang et al., Modern Physics Letters A 29, 1450067 (2014).

[4] R. Kantowski \& R. K. Sachs, Journal of Mathematical Physics 7, 443 (1966).

[5] G. F. R. Ellis \& H. van Elst, Cosmological Models (Cargèse lectures 1998) in NATO Advanced Science Institutes (ASI) Series C 541, 1 (1999).

[6] C. Clarkson, Comptes Rendus Physique 13, 682 (2012).

[7] A. R. Liddle, An Introduction to Modern Cosmology (Chichester, UK, 2003).

[8] A. Ashtekar et al., ArXiv e-prints 1409.5823 (2014).

[9] C. M. Will, Living Reviews in Relativity 17, (2014).

[10] C. M. Will, Classical and Quantum Gravity 32, 120201 (2015).

[11] S. Capozziello \& V. Faraoni, Beyond Einstein Gravity. A survey of Gravitational Theories for Cosmology and Astrophysics (Springer Science+Business Media, 2011).

[12] T. P. Sotiriou \& V. Faraoni, Reviews of Modern Physics 82, 451 (2010).

[13] A. de Felice \& S. Tsujikawa, Living Reviews in Relativity 13, 3 (2010).

[14] R. A. D’Inverno, Introducing Einstein's relativity (Intern. Elektron. Rundschau, 1992).

[15] J. V. Narlikar, An Introduction to Relativity (Cambridge University Press, 2010).

[16] J. Plebanski \& A. Krasiński, An Introduction to General Relativity and Cosmology (Cambridge University Press, 2006).

[17] M. P. Hobson et al., General Relativity (Cambridge University Press, 2006).

[18] R. M. Wald, General Relativity (University of Chicago Press, 1984).

[19] S. Dodelson, Modern Cosmology (Amsterdam Academic Press, 2003).

[20] P. Peter \& J. Uzan, Primordial Cosmology (Oxford University Press, 2009).

[21] V. Mukhanov, Physical Foundations of Cosmology (Cambridge University Press, 2005). 
[22] S. Weinberg, Cosmology (Oxford University Press, 2008).

[23] K. Olive \& P. D. Group, Chinese Physics C 38, 090001 (2014).

[24] E. Kolb \& M. Turner, The Early Universe (Westview Press, 1994).

[25] R. A. Alpher et al., Physical Review 74, 1198 (1948).

[26] J. F. Lara et al., Physical Review D 73, 083501 (2006).

[27] B. D. Fields, Annual Review of Nuclear and Particle Science 61, 47 (2011).

[28] M. Regis \& C. Clarkson, General Relativity and Gravitation 44, 567 (2010).

[29] G. Gamow, Nature 162, 680 (1948).

[30] Planck Collaboration et al., ArXiv e-prints 1502.01595 (2015).

[31] A. Loeb, Astrophysical Journal Letters 471, L1 (1996).

[32] B. Bassett \& R. Hlozek, in Dark Energy: Observational and Theoretical Approaches, (Cambridge University Press, 2010).

[33] P. J. E. Peebles, Astrophysical Journal Letters 263, L1 (1982).

[34] G. R. Blumenthal et al., Nature 311, 517 (1984).

[35] M. Davis et al., Nature 356, 489 (1992).

[36] F. Bernardeau et al., Physics Reports 367, 1 (2002).

[37] E. Hubble, Proceedings of the National Academy of Science 15, 168 (1929).

[38] S. Webb, in Measuring the Universe: The Cosmological Distance Ladder (SpringerPraxis Series in Astronomy and Astrophysics, 1999).

[39] W. Freedman et al., in New Cosmological Data and the Values of the Fundamental Parameters, Vol. 201 of IAU Symposium, (Cambridge University Press, 2005).

[40] B. Schmidt et al., IAU Circulars 5984, (1994).

[41] J. L. Tonry \& M. Franx, Astrophysical Journal 515, 512 (1999).

[42] C. D. Fassnacht et al., in Gravitational Lensing: Recent Progress and Future Go, Vol. 237 of Astronomical Society of the Pacific Conference Series, (ASP, 2001).

[43] M. Birkinshaw, Physics Reports 310, 97 (1999).

[44] E. D. Reese et al., Astrophysical Journal 581, 53 (2002).

[45] D. N. Spergel et al., The Astrophysical Journal Supplement Series 148, 175 (2003).

[46] S. Perlmutter et al., Astrophysical Journal 517, 565 (1999).

[47] A. G. Riess et al., The Astronomical Journal 116, 1009 (1998). 
[48] A. G. Riess et al., Astrophysical Journal 699, 539 (2009).

[49] W. J. Percival et al., MNRAS 401, 2148 (2010).

[50] R. Amanullah et al., Astrophysical Journal 716, 712 (2010).

[51] D. Larson et al., The Astrophysical Journal, Supplement 192, 16 (2011).

[52] E. Komatsu et al., The Astrophysical Journal, Supplement 192, 18 (2011).

[53] J. J. Cowan et al., Annual Review of Astronomy and Astrophysics 29, 447 (1991).

[54] R. Cayrel et al., Nature 409, 691 (2001).

[55] B. Chaboyer, Physics Reports 307, 23 (1998).

[56] R. G. Gratton et al., Astrophysical Journal 491, 749 (1997).

[57] F. Pont et al., Astronomy \& Astrophysics 329, 87 (1998).

[58] C. A. Egan, Ph.D. thesis, School of Physics, The University of New South Wales, Sydney NSW, Australia, 2010.

[59] S. Weinberg, Gravitation and Cosmology: Principles and Applications of the General Theory of Relativity (John Wiley \& Sons, 1972).

[60] W. Rindler, Monthly Notices of the Royal Astron. Society 116, 662 (1956).

[61] C. W. Misner et al., Gravitation (San Francisco: W.H. Freeman and Co., 1973).

[62] J. P. Preskill, Physical Review Letters 43, 1365 (1979).

[63] D. S. Goldwirth \& T. Piran, Physics Reports 214, 223 (1992).

[64] T. W. B. Kibble, Journal of Physics A Mathematical General 9, 1387 (1976).

[65] Y. B. Zeldovich \& M. Y. Khlopov, Physics Letters B 79, 239 (1978).

[66] T. W. B. Kibble, Physics Reports 67, 183 (1980).

[67] M. Novello \& S. E. P. Bergliaffa, Physics Reports 463, 127 (2008).

[68] R. Jimenez et al., Monthly Notices of the Royal Astron. Society 282, 926 (1996).

[69] H. B. Richer et al., Astrophysical Journal Letters 574, L151 (2002).

[70] B. M. S. Hansen et al., Astrophysical Journal Letters 574, L155 (2002).

[71] E. J. Copeland et al., International Journal of Modern Physics D 15, 1753 (2006).

[72] B. E. Schaefer, Astrophysical Journal 660, 16 (2007).

[73] H. J. Mosquera Cuesta et al., astro-ph/0609262 (2006).

[74] S. Carroll, in APS April Meeting Abstracts (American Physical Society, 2006). 
[75] M. Novello et al., Physical Review D 69, 127301 (2004).

[76] M. Makler et al., Physics Letters B 555, 1 (2003).

[77] R. Maartens, in The Invisible Universe: Dark Matter and Dark Energy, Vol. 720 of Lecture Notes in Physics, (Berlin Springer Verlag, 2007).

[78] S. Nojiri \& S. D. Odintsov, hep-th/0601213 (2006).

[79] S. E. Perez Bergliaffa, Physics Letters B 642, 311 (2006).

[80] W. Hu \& I. Sawicki, Physical Review D 76, 064004 (2007).

[81] S. Appleby \& R. Battye, Physics Letters B 654, 7 (2007).

[82] A. A. Starobinsky, Soviet Journal of Exper. and Theor. Physics Letters 86, 157 (2007).

[83] D. Hooper \& E. A. Baltz, Annual Review of Nuclear and Particle Science 58, 293 (2008).

[84] B. Russell, History of Western Philosophy (Unwin University Books, 1971).

[85] W. L. Freedman, Physica Scripta Volume T 85, 37 (2000).

[86] C. L. Bennett et al., Astrophysical Journal 436, 423 (1994).

[87] C. A. Clarkson, Ph.D. thesis, The University of Glasgow, UK, 2000.

[88] M. Quartin \& L. Amendola, Physical Review D 81, 043522 (2010).

[89] C. Clarkson et al., Physical Review Letters 101, 011301 (2008).

[90] R. R. Caldwell \& A. Stebbins, Physical Review Letters 100, 191302 (2008).

[91] T. Clifton et al., Physical Review Letters 101, 131302 (2008).

[92] T. Buchert \& J. Ehlers, Astronomy \& Astrophysics 320, 1 (1997).

[93] A. Ishibashi \& R. M. Wald, Classical and Quantum Gravity 23, 235 (2006).

[94] J. Grande \& L. Perivolaropoulos, Physical Review D 84, 023514 (2011).

[95] W. Valkenburg, Journal of Cosmology and Astroparticle Physics 1, 047 (2012).

[96] G. F. R. Ellis et al., Physics Reports 124, 315 (1985).

[97] R. Maartens, Ph.D. thesis, University of Cape Town, South Africa, 1980.

[98] H. Bondi, Monthly Notices of the Royal Astron. Society 107, 410 (1947).

[99] K. Tomita, Astrophysical Journal 529, 38 (2000).

[100] K. Tomita, Astrophysical Journal 529, 26 (2000).

[101] K. Tomita, Progress of Theoretical Physics 106, 929 (2001).

[102] K. Tomita, Progress of Theoretical Physics 105, 419 (2001). 
[103] K. Tomita, Monthly Notices of the Royal Astron. Society 326, 287 (2001).

[104] K. Tomita, Progress of Theoretical Physics 108, 103 (2002).

[105] K. Tomita, Astrophysical Journal 584, 580 (2003).

[106] A. G. Lemaître, General Relativity and Gravitation 29, 641 (1997), original title: "L’Univers en expansion", Annales de la Société Scientifique de Bruxelles A53, 51 (1933); Translated by M. A. H. MacCallum.

[107] R. C. Tolman, Proceedings of the National Academy of Science 20, 169 (1934).

[108] A. Krasiński, Inhomogeneous Cosmological Models (Cambridge University Press, 1997).

[109] K. Bolejko et al., in Structures in the Universe by Exact Methods: Formation, Evolution, Interactions, (Cambridge University Press, 2009).

[110] P. Szekeres, Communications in Mathematical Physics 41, 55 (1975).

[111] P. Szekeres, Physical Review D 12, 2941 (1975).

[112] A. Krasiński \& C. Hellaby, Physical Review D 65, 023501 (2002).

[113] A. Krasiński \& C. Hellaby, Physical Review D 69, 023502 (2004).

[114] A. Krasiński \& C. Hellaby, Physical Review D 69, 043502 (2004).

[115] C. Hellaby \& A. Krasiński, Physical Review D 73, 023518 (2006).

[116] K. Bolejko et al., Monthly Notices of the Royal Astron. Society 362, 213 (2005).

[117] P. S. Joshi \& I. H. Dwivedi, Physical Review D 47, 5357 (1993).

[118] M.-N. Célérier, astro-ph/0702416 (2007).

[119] T. Buchert, in Proceedings of 9th Workshop on General Relativity and Gravitation conference (JGRG9, 2000).

[120] T. Buchert, General Relativity and Gravitation 40, 467 (2008).

[121] C. Hellaby \& K. Lake, Astrophysical Journal 282, 1 (1984).

[122] C. Hellaby \& K. Lake, Astrophysical Journal 290, 381 (1985).

[123] W. Rindler \& D. Suson, Astronomy \& Astrophysics 218, 15 (1989).

[124] L. J. Goicoechea \& J. M. Martin-Mirones, Astronomy \& Astrophysics 186, 22 (1987).

[125] J. Schneider \& M.-N. Célérier, Astronomy and Astrophysics 348, 25 (1999).

[126] M.-N. Célérier, Astronomy \& Astrophysics 362, 840 (2000).

[127] R. Maartens et al., Classical and Quantum Gravity 13, 253 (1996).

[128] N. P. Humphreys et al., Astrophysical Journal 477, 47 (1997). 
[129] H. Alnes \& M. Amarzguioui, Physical Review D 74, 103520 (2006).

[130] H. Alnes \& M. Amarzguioui, Physical Review D 75, 023506 (2007).

[131] K. Enqvist \& T. Mattsson, Journal of Cosmology and Astroparticle Physics 2, 019 (2007).

[132] M. Blomqvist \& E. Mörtsell, Journal of Cosmology and Astroparticle Physics 5, 006 (2010).

[133] C. Clarkson \& M. Regis, Journal of Cosmology and Astroparticle Physics 2, 13 (2011).

[134] P. D. Lasky \& K. Bolejko, Classical and Quantum Gravity 27, 035011 (2010).

[135] H. Stephani, Communications in Mathematical Physics 4, 137 (1967).

[136] M. P. Dabrowski, Journal of Mathematical Physics 34, 1447 (1993).

[137] C. Hellaby \& A. Krasiński, Physical Review D 77, 023529 (2008).

[138] D. A. Szafron, Journal of Mathematical Physics 18, 1673 (1977).

[139] C. Quercellini et al., ArXiv:1011.2646 (2010).

[140] W. C. Lim et al., Journal of Cosmology and Astroparticle Physics 10, 10 (2013).

[141] G. C. Omer, Proceedings of the National Academy of Science 53, 1 (1965).

[142] H. Nariai, Sci. Rep. Tohoku Univ. Eighth Ser. 34, (1950).

[143] N. Hidekazu, Sci. Rep. Tohoku Univ. Eighth Ser. 35, (1951).

[144] R. A. Sussman, ArXiv e-prints (2007).

[145] J. T. Firouzjaee \& R. Mansouri, General Relativity and Gravitation 42, 2431 (2010).

[146] J. T. Firouzjaee \& R. Mansouri, EPL (Europhysics Letters) 97, 29002 (2012).

[147] J. T. Firouzjaee et al., General Relativity and Gravitation 44, 639 (2012).

[148] C. Kiefer et al., Physical Review D 75, 124010 (2007).

[149] C. Vaz \& L. Witten, General Relativity and Gravitation 43, 3429 (2011).

[150] K. Bolejko, Monthly Notices of the Royal Astron. Society 370, 924 (2006).

[151] K. Bolejko \& P. D. Lasky, Monthly Notices of the Royal Astron. Society 391, L59 (2008).

[152] C. Hellaby \& A. Krasiński, Physical Review D 66, 084011 (2002).

[153] A. Krasiński, Physical Review D 78, 064038 (2008).

[154] W. B. Bonnor, Communications in Mathematical Physics 51, 191 (1976).

[155] J. D. Barrow \& J. Stein-Schabes, Physics Letters A 103, 315 (1984). 
[156] C. Hellaby, Classical and Quantum Gravity 13, 2537 (1996).

[157] A. Sandage, Astrophysical Journal 136, 319 (1962).

[158] G. C. McVittie, Astrophysical Journal 136, 334 (1962).

[159] C. Quercellini et al., Physical Review Letters 102, 151302 (2009).

[160] L. Amendola et al., Journal of Cosmology and Astroparticle Physics 12, 42 (2013).

[161] S. Räsänen, Journal of Cosmology and Astroparticle Physics 3, 35 (2014).

[162] L. Amendola et al., Physics Letters B 660, 81 (2008).

[163] C. Quercellini et al., Monthly Notices of the Royal Astron. Society 391, 1308 (2008).

[164] A. Loeb, Astrophysical Journal Letters 499, L111 (1998).

[165] J. Darling, Astrophysical Journal Letters 761, L26 (2012).

[166] J.-P. Uzan et al., Physical Review Letters 100, 191303 (2008).

[167] M. Martinelli et al., Physical Review D 86, 123001 (2012).

[168] B. Moraes \& D. Polarski, Physical Review D 84, 104003 (2011).

[169] P.-S. Corasaniti et al., Physical Review D 75, 062001 (2007).

[170] H. Zhang et al., Physical Review D 76, 123508 (2007).

[171] J. Zhang et al., Physics Letters B 691, 11 (2010).

[172] D. Jain \& S. Jhingan, Physics Letters B 692, 219 (2010).

[173] P. Dunsby et al., Journal of Cosmology and Astroparticle Physics 6, 017 (2010).

[174] C.-M. Yoo et al., Journal of Cosmology and Astroparticle Physics 7, 012 (2010).

[175] P. Mishra et al., Physical Review D 86, 083520 (2012).

[176] A. Balcerzak \& M. P. Dabrowski, Physical Review D 87, 063506 (2013).

[177] A. Balcerzak, in American Institute of Physics Conference Series, Vol. 1514 of American Institute of Physics Conference Series, (AIP Conference Proceedings, 2013).

[178] A. Balcerzak \& M. P. Dabrowski, Journal of Cosmology and Astroparticle Physics 6, 35 (2014).

[179] S. M. Koksbang \& S. Hannestad, Journal of Cosmology and Astroparticle Physics 1, 009 (2016).

[180] C.-M. Yoo et al., Physical Review D 83, 043527 (2011).

[181] G. F. R. Ellis, in General Relativity and Cosmology, (Cambridge University Press, 1971). 
[182] J. Ehlers, Abh. Akad. Wiss. Lit. Mainz. Nat. 11, 793 (1991), [Traducción al inglés: G. F. R. Ellis and P. K. S. Dunsby Gen. Rel. Grav. 25, 1225-1266 (1993)].

[183] P. Mishra et al., ArXiv: 1301.4358 (2013).

[184] P. Mishra \& M.-N. Célérier, ArXiv: 1403.5229 (2014).

[185] M. Fontanini et al., Physical Review D 80, 123515 (2009).

[186] L. Witten, Gravitation: An Introduction to Current Research (New York: Wiley, 1962).

[187] J. R. Ellis \& K. A. Olive, Nature 303, 679 (1983).

[188] H. S. Hwang \& M. G. Lee, Astrophysical Journal 662, 236 (2007).

[189] J. D. Barrow et al., Monthly Notices of the Royal Astron. Society 213, 917 (1985).

[190] L.-X. Li, General Relativity and Gravitation 30, 497 (1998).

[191] M. Visser, General Relativity and Gravitation 37, 1541 (2005).

[192] F. A. Teppa Pannia \& S. E. Perez Bergliaffa, Journal of Cosmology and Astroparticle Physics 8, 30 (2013).

[193] S. Capozziello et al., Physical Review D 84, 124061 (2011).

[194] J. Liske et al., Monthly Notices of the Royal Astron. Society 386, 1192 (2008).

[195] L. Pasquini et al., The Messenger 122, 10 (2005).

[196] K. Yagi \& N. Seto, Physical Review D 83, 044011 (2011).

[197] A. H. Guth, Physical Review D 23, 347 (1981).

[198] A. D. Linde, Physics Letters B 108, 389 (1982).

[199] A. Albrecht \& P. J. Steinhardt, Physical Review Letters 48, 1220 (1982).

[200] A. D. Linde, Physics Letters B 114, 431 (1982).

[201] A. D. Linde, Physics Letters B 116, 335 (1982).

[202] A. A. Starobinsky, Physics Letters B 117, 175 (1982).

[203] A. D. Linde, Physics Letters B 129, 177 (1983).

[204] A. D. Linde, Soviet Journal of Exper. and Theor. Physics Letters 38, 176 (1983).

[205] A. S. Goncharov \& A. D. Linde, Zhurnal Eksperim. i Teoret. Fiziki 86, 1594 (1984).

[206] A. S. Goncharov \& A. D. Linde, Physics Letters B 139, 27 (1984).

[207] A. D. Linde, Physics Letters B 175, 395 (1986).

[208] E. B. Gliner, Zh. Eksp. Teor. Fiz. 49, 542 (1965), [Traducción al inglés: Sov. Phys. JETP $22,378(1966)]$. 
[209] A. D. Linde, Soviet Journal of Exper. and Theor. Physics Letters 19, 183 (1974).

[210] M. Veltman, Physical Review Letters 34, 777 (1975).

[211] J. Dreitlein, Physical Review Letters 33, 1243 (1974).

[212] R. Brout et al., Annals of Physics 115, 78 (1978).

[213] R. Brout et al., General Relativity and Gravitation 10, 1 (1979).

[214] A. A. Starobinsky, Physics Letters B 91, 99 (1980).

[215] D. Kazanas, Astrophysical Journal Letters 241, L59 (1980).

[216] K. Sato, Monthly Notices of the Royal Astron. Society 195, 467 (1981).

[217] A. Albrecht et al., Physical Review Letters 48, 1437 (1982).

[218] A. D. Dolgov \& A. D. Linde, Physics Letters B 116, 329 (1982).

[219] I. B. Zeldovich \& I. D. Novikov, Relativistic Astrophysics. Volume 2 - The Structure and Evolution of the Universe (University of Chicago Press, 1983).

[220] A. Linde, ArXiv: hep-th/0503203 (2005).

[221] D. H. D. H. Lyth \& A. A. Riotto, Physics Reports 314, 1 (1999).

[222] F. Hoyle \& J. V. Narlikar, Proceedings of the Royal Society of London Series A 273, 1 (1963).

[223] G. W. Gibbons \& S. W. Hawking, Physical Review D 15, 2738 (1977).

[224] S. W. Hawking \& I. L. Moss, Physics Letters B 110, 35 (1982).

[225] R. M. Wald, Physical Review D 28, 2118 (1983).

[226] T. Piran, in Numerical relativity and cosmology, (NATO Advanced Science Institutes (ASI) Series C, 1988)Vol. 219, pp. 261-282.

[227] D. S. Goldwirth \& T. Piran, Physical Review Letters 64, 2852 (1990).

[228] D. S. Goldwirth \& T. Piran, Physical Review D 40, 3263 (1989).

[229] N. Deruelle \& D. S. Goldwirth, Physical Review D 51, 1563 (1995).

[230] R. S. Perez \& N. Pinto-Neto, Gravitation and Cosmology 17, 136 (2011).

[231] V. A. Berezin et al., Physical Review D 36, 2919 (1987).

[232] H. Sato, Progress of Theoretical Physics 76, 1250 (1986).

[233] A. Aguirre \& M. C. Johnson, Physical Review D 72, 103525 (2005).

[234] W. Fischler et al., Journal of High Energy Physics 5, 41 (2008).

[235] D. Simon et al., Journal of Cosmology and Astroparticle Physics 11, 8 (2009). 
[236] K. Maeda \& H. Sato, Progress of Theoretical Physics 70, 772 (1983).

[237] K. Maeda \& H. Sato, Progress of Theoretical Physics 70, 1276 (1983).

[238] A. Rakić et al., Annalen der Physik 522, 336 (2010).

[239] W. Israel, Il Nuovo Cimento B Series 10 44, 1 (1966).

[240] N. Sen, Annalen der Physik 378, 365 (1924).

[241] K. Lanczos, Annalen der Physik 379, 518 (1924).

[242] A. H. Taub, Journal of Mathematical Physics 21, 1423 (1980).

[243] C. Barrabes, Classical and Quantum Gravity 6, 581 (1989).

[244] C. Barrabès \& W. Israel, Physical Review D 43, 1129 (1991).

[245] M. Visser, Lorentzian Wormholes. From Einstein to Hawking (Woodbury, N.Y.: American Institute of Physics, 1995).

[246] Ø. Grøn \& S. Hervik, Einstein's General Theory of Relativity with Modern Applications in Cosmology (Springer Science+Business Media, 2007).

[247] N. Sakai \& K.-I. Maeda, Physical Review D 50, 5425 (1994).

[248] A. H. A. Alfedeel \& C. Hellaby, General Relativity and Gravitation 42, 1935 (2010).

[249] W. Schiesser, The Numerical Method of Lines: Integration of Partial Differential Equations (Academic Press, 1991).

[250] A. Rakic et al., in Cosmic Structure and Evolution (PoS Cosmology, 2009).

[251] K. Bolejko \& J. S. B. Wyithe, Journal of Cosmology and Astroparticle Physics 2, 020 (2009).

[252] H. Alnes et al., Physical Review D 73, 083519 (2006).

[253] T. Clifton et al., Journal of Cosmology and Astroparticle Physics 7, 029 (2009).

[254] J. P. Zibin et al., Physical Review Letters 101, 251303 (2008).

[255] T. Biswas et al., Journal of Cosmology and Astroparticle Physics 11, 030 (2010).

[256] A. Moss et al., Physical Review D 83, 103515 (2011).

[257] K. Tomita \& K. T. Inoue, Physical Review D 79, 103505 (2009).

[258] R. Maartens et al., Physical Review D 59, 083506 (1999).

[259] V. Marra \& M. Pääkkönen, Journal of Cosmology and Astroparticle Physics 1, 25 (2012).

[260] V. Marra et al., Monthly Notices of the Royal Astron. Society 431, 1891 (2013).

[261] R. K. Sachs \& A. M. Wolfe, Astrophysical Journal 147, 73 (1967). 
[262] P. K. S. Dunsby, Classical and Quantum Gravity 14, 3391 (1997).

[263] A. Challinor \& A. Lasenby, Physical Review D 58, 023001 (1998).

[264] J. Ehlers, in General Relativity and Cosmology, (Cambridge University Press, 1971).

[265] R. W. Lindquist, Annals of Physics 37, 487 (1966).

[266] W. Israel, in General Relativity, (Clarendon Press, Oxford, 1972).

[267] J. Stewart, Non-Equilibrium Relativistic Kinetic Theory, Vol. 10 of Lecture Notes in Physics (Springer, 1971).

[268] J. D. Jackson, Classical Electrodynamics (New York: Wiley, 1962).

[269] R. P. Feynman, Feynman lectures on physics - Volume 1 (Reading, Ma.: Addison-Wesley, 1963).

[270] G. Ellis et al., Annals of Physics 150, 455 (1983).

[271] G. Ellis et al., Annals of Physics 150, 487 (1983).

[272] W. C. Lim et al., Physical Review D 79, 123526 (2009).

[273] S. February et al., Journal of Cosmology and Astroparticle Physics 3, 23 (2013).

[274] C. Pitrou et al., Physical Review D 78, 063526 (2008).

[275] H. Goto \& H. Kodama, ArXiv 1002.3161 (2010).

[276] H. van Elst \& C. Uggla, Classical and Quantum Gravity 14, 2673 (1997).

[277] G. F. R. Ellis \& H. van Elst, in On Einstein's Path: essays in honor of Engelbert Schucking, (Springer, 1999).

[278] A. Raychaudhuri, Phys. Rev. 98, 1123 (1955).

[279] D. Kramer \& E. Schmutzer, Exact Solutions of Einstein's Field Equations (Cambridge University Press, 1980).

[280] A. Krasinski, General Relativity and Gravitation 15, 673 (1983).

[281] J. V. Narlikar, Introduction to Cosmology (Jones and Bartlett, 1983).

[282] A. Balcerzak et al., Astrophysical Journal 792, 92 (2014).

[283] M. P. Dabrowski, Astrophysical Journal 447, 43 (1995).

[284] M. P. Da̧browski \& M. A. Hendry, Astrophysical Journal 498, 67 (1998).

[285] C. A. Clarkson \& R. K. Barrett, Classical and Quantum Gravity 16, 3781 (1999).

[286] R. K. Barrett \& C. A. Clarkson, Classical and Quantum Gravity 17, 5047 (2000).

[287] J. Stelmach \& I. Jakacka, Classical and Quantum Gravity 18, 2643 (2001). 
[288] P. S. Wesson \& J. Ponce de Leon, Physical Review D 39, 420 (1989).

[289] C. Bona \& B. Coll, General Relativity and Gravitation 20, 297 (1988).

[290] R. A. Sussman, General Relativity and Gravitation 32, 1527 (2000).

[291] D. Lorenz-Petzold, Journal of Astrophysics and Astronomy 7, 155 (1986).

[292] A. Balbi \& C. Quercellini, Monthly Notices of the Royal Astron. Society 382, 1623 (2007).

[293] P. Mishra \& M.-N. S. Célérier, Tejinder P., in Thirteenth Marcel Grossmann Meeting: On Recent Developments in Theoretical and Experimental General Relativity, Astrophysics and Relativistic Field Theories, (World Scientific, 2015).

[294] S. E. Perez Bergliaffa, in Proceedings of the XIV Brazilian School of Cosmology and Gravitation (Cambridge University Press, 2010).

[295] F. A. Teppa Pannia et al., Enviado a General Relativity and Gravitation para ser sometido a arbitraje. Febrero (2016).

[296] T. P. Sotiriou \& S. Liberati, Annals of Physics 322, 935 (2007).

[297] V. Miranda et al., Physical Review Letters 102, 221101 (2009).

[298] L. G. Jaime et al., Physical Review D 83, 024039 (2011).

[299] S. Nojiri \& S. D. Odintsov, Physical Review D 74, 086005 (2006).

[300] S. E. Perez Bergliaffa, in The Eleventh Marcel Grossmann Meeting: On Recent Developments in Theoretical and Experimental General Relativity, Gravitation and Relativistic Field Theories, (World Scientific, 2008).

[301] M. Orellana et al., General Relativity and Gravitation 45, 771 (2013).

[302] E. V. Linder, Physical Review D 80, 123528 (2009).

[303] R. Kerner, General Relativity and Gravitation 14, 453 (1982).

[304] S. Capozziello et al., Physical Review D 78, 063504 (2008).

[305] M. Martinelli et al., Physical Review D 85, 024006 (2012).

[306] L. Yang et al., Physical Review D 82, 103515 (2010).

[307] S. Capozziello \& M. de Laurentis, Physics Reports 509, 167 (2011). 